NATIONAL LABORATORY

MANAGED BY UT-BATTELLE

FOR THE DEPARTMENT OF ENERGY

\title{
Data Compilation for AGR-2 UCO Variant Compact Lot LEU09-OP2-Z
}

\author{
Revision 1
}

\author{
John D. Hunn, Fred C. Montgomery and \\ Peter J. Pappano
}

February 2010

This document has been reviewed and is determined to be APPROVED FOR PUBLIC RELEASE.

Name/Title: Leesa Laymance/ORNL TI0

Date: 5/19/2020
Prepared for the United States Department of Energy Office of Nuclear Energy under the Next Generation Nuclear Plant Advanced Gas Reactor Fuel Development Program

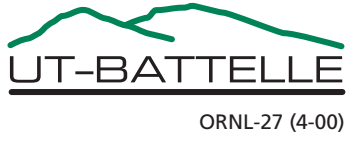




\section{DOCUMENT AVAILABILITY}

Reports produced after January 1, 1996, are generally available free via the U.S. Department of Energy (DOE) Information Bridge.

Web site http://www.osti.gov/bridge

Reports produced before January 1, 1996, may be purchased by members of the public from the following source.

National Technical Information Service

5285 Port Royal Road

Springfield, VA 22161

Telephone 703-605-6000 (1-800-553-6847)

TDD 703-487-4639

Fax 703-605-6900

E-mail info@ntis.fedworld.gov

Web site http://www.ntis.gov/support/ordernowabout.htm

Reports are available to DOE employees, DOE contractors, Energy Technology Data Exchange (ETDE) representatives, and International Nuclear Information System (INIS)

representatives from the following source.

Office of Scientific and Technical Information

P.O. Box 62

Oak Ridge, TN 37831

Telephone 865-576-8401

Fax 865-576-5728

E-mail reports@adonis.osti.gov

Web site http://www.osti.gov/contact.html

This report was prepared as an account of work sponsored by an agency of the United States Government. Neither the United States Government nor any agency thereof, nor any of their employees, makes any warranty, express or implied, or assumes any legal liability or responsibility for the accuracy, completeness, or usefulness of any information, apparatus, product, or process disclosed, or represents that its use would not infringe privately owned rights. Reference herein to any specific commercial product, process, or service by trade name, trademark, manufacturer, or otherwise, does not necessarily constitute or imply its endorsement, recommendation, or favoring by the United States Government or any agency thereof. The views and opinions of authors expressed herein do not necessarily state or reflect those of the United States Government or any agency thereof. 


\title{
Data Compilation for AGR-2 UCO Variant Compact Lot LEU09-OP2-Z
}

\author{
J. D. Hunn, F. C. Montgomery and P. J. Pappano \\ Oak Ridge National Laboratory
}

This document is a compilation of characterization data for the candidate AGR-2 UCO Variant fuel compact lot LEU09-OP2-Z. The compacts were produced by ORNL for the Advanced Gas Reactor Fuel Development and Qualification (AGR) program for the second AGR irradiation test (AGR-2). This compact lot was fabricated using particle composite LEU09. LEU09 came from Babcock and Wilcox (B\&W) coated particle lot G73J-14-93073A, which was an upgraded batch of TRISO-coated $425 \mu \mathrm{m}$ diameter, $14 \%$ low enriched uranium oxide/uranium carbide kernels (LEUCO). The AGR-2 TRISO-coated particles consist of a spherical kernel coated with an $\sim 50 \%$ dense carbon buffer layer (100 $\mu \mathrm{m}$ nominal thickness), followed by a dense inner pyrocarbon layer $(40 \mu \mathrm{m}$ nominal thickness), followed by a $\mathrm{SiC}$ layer $(35 \mu \mathrm{m}$ nominal thickness), followed by another dense outer pyrocarbon layer (40 $\mu \mathrm{m}$ nominal thickness). The kernels were also manufactured by B\&W and identified as kernel lot G73I-14-69307. Two data packages were submitted by $\mathrm{B} \& \mathrm{~W}$ containing the acceptance testing results for the kernels and coated particles, these are identified by their lot numbers. A discussion on the coating of the B\&W TRISO particles can also be found in INL report INL/EXT-09-16545. A data compilation of ORNL analysis of G73J-14-93073A can be found in ORNL/TM-2008/134.

The AGR-2 Fuel Specification (INL SPC-923) provides the requirements necessary for acceptance of the fuel manufactured for the AGR-2 irradiation test. Section 3.3 of SPC-923 provides the property requirements for the heat treated compacts. The Statistical Sampling Plan for AGR-2 Fuel Materials (INL PLN-2691) provides additional guidance regarding statistical methods for product acceptance and recommended sample sizes. The procedures for characterizing and qualifying the compacts are outlined in ORNL product inspection plan AGRCHAR-PIP-12. The inspection report forms generated by this product inspection plan document the product acceptance for the property requirements listed in section 3.3 of SPC-923. Prior to compacting, the overcoated particles are characterized per ORNL product inspection plan AGRCHAR-PIP-11 to obtain data needed for calculation of compacting charge weight and matrix density. Riffling of compact charges is also covered by this procedure. Prior to overcoating, the TRISO particles are characterized per ORNL product inspection plan AGR-CHAR-PIP-10 to determine uranium content, obtain data needed for overcoating and compact fabrication, and obtain further data needed for calculation of matrix density. Riffling of overcoater charges is also covered by this procedure. This document contains all the inspection report forms and data report forms generated by these inspection plans.

In addition to the characterization data, this report also contains other records relevant to the fuel product acceptance. A history of the material flow and sample naming is included. The overcoating and compacting process is summarized, and a record of the materials used to make the matrix is included. A Certificate of Conformance and any applicable Nonconformance Reports are attached as Appendices. 


\section{Table of Contents}

1 Material identification record for LEU09-OP2-Z compacts __ 5

2 Summary of acceptance test results for LEU09-OP2-Z_

3 Compacting process conditions__ 10

4 Impurity analysis of matrix, resin, and graphites_ 20

5 Characterization of coated particles _ 25

6 Characterization of overcoated particles__ 33

7 Characterization of compacts

Appendix A: Certificate of Conformance _ 123

Appendix B: Nonconformance Reports 125

Appendix C: Upgrading of LEU09 using a Roller-micrometer___ 130 


\section{Material identification record for LEU09-OP2-Z compacts}

Table 1-1 lists the materials used to make the LEU09-OP2-Z compacts, including intermediate batches and samples used for characterization. TRISO-coated particles were shipped from B\&W to ORNL on April 23, 2009. Forty eight completed compacts were shipped to INL on October 28, 2009. Twelve compacts were retained at ORNL and 107 compacts were consumed at ORNL by the QC acceptance testing. Table 1-2 lists the disposition of each compact.

Table 1-1: Material identification record for LEU09-OP2-Z compacts

\begin{tabular}{|c|c|c|c|}
\hline Sample ID & Parent material & \multicolumn{2}{|l|}{ Notes } \\
\hline G73I-14-69307 & $\begin{array}{l}\text { G73I-14-59370 } \\
\text { G73I-14-59371 } \\
\text { G73I-14-59372 } \\
\text { G73I-14-59373 } \\
\text { G73I-14-59375 }\end{array}$ & \multicolumn{2}{|c|}{ B\&W kernel composite from 5 batches } \\
\hline G73J-14-93073A & G73I-14-69307 & \multicolumn{2}{|c|}{ B\&W TRISO-coated particles } \\
\hline $\begin{array}{l}\text { NP-B8453 } \\
\text { NP-B8454 } \\
\text { NP-B8455 } \\
\text { NP-B8456 }\end{array}$ & G73J-14-93073A & \multicolumn{2}{|c|}{$999.9 \mathrm{~g}$ sample from $93073 \mathrm{~A}$ shipped to ORNL on $4 / 23 / 2009$} \\
\hline "LEU09 & $\begin{array}{l}\text { NP-B8453 } \\
\text { NP-B8454 } \\
\text { NP-B8455 } \\
\text { NP-B8456 }\end{array}$ & \multicolumn{2}{|c|}{$\begin{array}{l}\text { TRISO-coated particles re-composited, washed with methanol, and } \\
\text { sorted by roller-micrometer to remove } 0.0127 \mathrm{~g} \text { of the smallest } \\
\text { (faceted) particles }\end{array}$} \\
\hline LEU09-A01 & LEU09 & \multicolumn{2}{|c|}{ TRISO-coated particle QC archive } \\
\hline $\begin{array}{l}\text { LEU09-B01 } \\
\text { LEU09-C01 } \\
\text { LEU09-D01 } \\
\text { LEU09-E01 } \\
\text { LEU09-F01 } \\
\text { LEU09-G01 }\end{array}$ & LEU09 & \multicolumn{2}{|c|}{ TRISO-coated particle characterization samples } \\
\hline LEU09-Y\#\# & LEU09 & \multicolumn{2}{|c|}{$\begin{array}{l}\text { Charges for overcoating, numbered Y01 through Y48 } \\
\text { LEU09-Y42 to LEU09-Y48 were not overcoated }\end{array}$} \\
\hline RD13371 & Asbury Graphite Mills & \multicolumn{2}{|l|}{ Natural graphite } \\
\hline KRB2000 & SGL Carbon & \multicolumn{2}{|l|}{ Synthetic graphite } \\
\hline SC1008 & Hexion & \multicolumn{2}{|c|}{ Durite resin lot LK9DA0008 } \\
\hline $\begin{array}{l}\text { RDKrS-050809 } \\
\text { RDKrS-052109 } \\
\text { RDKrS-060109 }\end{array}$ & $\begin{array}{l}64 \mathrm{wt} \% \text { RD13371 } \\
16 \mathrm{wt} \% \text { KRB2000 } \\
20 \mathrm{wt} \% \text { SC1008 }\end{array}$ & \multicolumn{2}{|c|}{ Matrix precursor batches } \\
\hline LEU09-OP2 & \multicolumn{2}{|c|}{$\begin{array}{l}\text { LEU09-Y01 to LEU09-Y17 + RDKrS-050809 } \\
\text { LEU09-Y18 to LEU09-Y34 + RDKrS-052109 } \\
\text { LEU09-Y35 to LEU09-Y41 + RDKrS-060109 }\end{array}$} & Over-coated particle composite. \\
\hline LEU09-OP2-A01 & LEU09-OP2 & \multicolumn{2}{|c|}{ Overcoated particle QC archive } \\
\hline $\begin{array}{l}\text { LEU09-OP2-B01 } \\
\text { LEU09-OP2-C01 }\end{array}$ & LEU09-OP2 & \multicolumn{2}{|c|}{ Overcoated particle characterization samples } \\
\hline LEEU09-OP2-G\#\#\# & LEU09-OP2 & \multicolumn{2}{|c|}{ "Compacts, numbered G001 through G185 } \\
\hline LEU09-OP2-Z\#\#\# & LEU09-OP2-G\#\#\# & \multicolumn{2}{|c|}{$\begin{array}{l}\text { Compacts, numbered Z001 through Z167 } \\
\text { One to one correspondence to G\#\#\# recorded on DRF24C (section 7) }\end{array}$} \\
\hline
\end{tabular}


Table 1-2: Disposition of LEU09-OP2-Z compacts

\begin{tabular}{|c|c|c|c|c|}
\hline Sent to INL & Retained at ORNL & \multicolumn{3}{|c|}{ Consumed during QC analysis } \\
\hline LEU09-OP2-Z014 & $\begin{array}{l}\text { LEU09-OP2-Z002 } \\
\end{array}$ & \begin{tabular}{|l|} 
LEU09-OP2-Z001 \\
\end{tabular} & "LEU09-OP2-Z072 & LEU09-OP2-Z151 \\
\hline LEU09-OP2-Z015 & LEU09-OP2-Z004 & LEU09-OP2-Z003 & LEU09-OP2-Z073 & LEU09-OP2-Z152 \\
\hline LEU09-OP2-Z016 & LEU09-OP2-Z005 & LEU09-OP2-Z006 & LEU09-OP2-Z074 & LEU09-OP2-Z155 \\
\hline LEU09-OP2-Z017 & LEU09-OP2-Z038 & LEU09-OP2-Z007 & LEU09-OP2-Z076 & LEU09-OP2-Z157 \\
\hline LEU09-OP2-Z018 & LEU09-OP2-Z057 & LEU09-OP2-Z008 & LEU09-OP2-Z078 & LEU09-OP2-Z159 \\
\hline LEU09-OP2-Z022 & LEU09-OP2-Z069 & LEU09-OP2-Z009 & LEU09-OP2-Z080 & LEU09-OP2-Z160 \\
\hline LEU09-OP2-Z026 & LEU09-OP2-Z089 & LEU09-OP2-Z010 & LEU09-OP2-Z081 & LEU09-OP2-Z161 \\
\hline LEU09-OP2-Z028 & LEU09-OP2-Z102 & LEU09-OP2-Z011 & LEU09-OP2-Z084 & LEU09-OP2-Z162 \\
\hline LEU09-OP2-Z040 & LEU09-OP2-Z130 & LEU09-OP2-Z012 & LEU09-OP2-Z086 & LEU09-OP2-Z163 \\
\hline LEU09-OP2-Z043 & LEU09-OP2-Z139 & LEU09-OP2-Z013 & LEU09-OP2-Z087 & LEU09-OP2-Z164 \\
\hline LEU09-OP2-Z049 & LEU09-OP2-Z158 & LEU09-OP2-Z019 & LEU09-OP2-Z090 & LEU09-OP2-Z166 \\
\hline LEU09-OP2-Z053 & LEU09-OP2-Z165 & LEU09-OP2-Z020 & LEU09-OP2-Z091 & \\
\hline LEU09-OP2-Z056 & & LEU09-OP2-Z021 & LEU09-OP2-Z093 & \\
\hline LEU09-OP2-Z059 & & LEU09-OP2-Z023 & LEU09-OP2-Z094 & \\
\hline LEU09-OP2-Z062 & & LEU09-OP2-Z024 & LEU09-OP2-Z095 & \\
\hline LEU09-OP2-Z063 & & LEU09-OP2-Z025 & LEU09-OP2-Z096 & \\
\hline LEU09-OP2-Z066 & & LEU09-OP2-Z027 & LEU09-OP2-Z097 & \\
\hline LEU09-OP2-Z075 & & LEU09-OP2-Z029 & LEU09-OP2-Z098 & \\
\hline LEU09-OP2-Z077 & & LEU09-OP2-Z030 & LEU09-OP2-Z099 & \\
\hline LEU09-OP2-Z079 & & LEU09-OP2-Z031 & LEU09-OP2-Z101 & \\
\hline LEU09-OP2-Z082 & & LEU09-OP2-Z032 & LEU09-OP2-Z106 & \\
\hline LEU09-OP2-Z083 & & LEU09-OP2-Z033 & LEU09-OP2-Z107 & \\
\hline LEU09-OP2-Z085 & & LEU09-OP2-Z034 & LEU09-OP2-Z108 & \\
\hline LEU09-OP2-Z088 & & LEU09-OP2-Z035 & LEU09-OP2-Z109 & \\
\hline LEU09-OP2-Z092 & & LEU09-OP2-Z036 & LEU09-OP2-Z110 & \\
\hline LEU09-OP2-Z100 & & LEU09-OP2-Z037 & LEU09-OP2-Z111 & \\
\hline LEU09-OP2-Z103 & & LEU09-OP2-Z039 & LEU09-OP2-Z113 & \\
\hline LEU09-OP2-Z104 & & LEU09-OP2-Z041 & LEU09-OP2-Z115 & \\
\hline LEU09-OP2-Z105 & & LEU09-OP2-Z042 & LEU09-OP2-Z116 & \\
\hline LEU09-OP2-Z112 & & LEU09-OP2-Z044 & LEU09-OP2-Z117 & \\
\hline LEU09-OP2-Z114 & & LEU09-OP2-Z045 & LEU09-OP2-Z118 & \\
\hline LEU09-OP2-Z119 & & LEU09-OP2-Z046 & LEU09-OP2-Z120 & \\
\hline LEU09-OP2-Z124 & & LEU09-OP2-Z047 & LEU09-OP2-Z121 & \\
\hline LEU09-OP2-Z125 & & LEU09-OP2-Z048 & LEU09-OP2-Z122 & \\
\hline LEU09-OP2-Z126 & & LEU09-OP2-Z050 & LEU09-OP2-Z123 & \\
\hline LEU09-OP2-Z127 & & LEU09-OP2-Z051 & LEU09-OP2-Z133 & \\
\hline LEU09-OP2-Z128 & & LEU09-OP2-Z052 & LEU09-OP2-Z136 & \\
\hline LEU09-OP2-Z129 & & LEU09-OP2-Z054 & LEU09-OP2-Z137 & \\
\hline LEU09-OP2-Z131 & & LEU09-OP2-Z055 & LEU09-OP2-Z138 & \\
\hline LEU09-OP2-Z132 & & LEU09-OP2-Z058 & LEU09-OP2-Z140 & \\
\hline LEU09-OP2-Z134 & & LEU09-OP2-Z060 & LEU09-OP2-Z143 & \\
\hline LEU09-OP2-Z135 & & LEU09-OP2-Z061 & LEU09-OP2-Z144 & \\
\hline LEU09-OP2-Z141 & & LEU09-OP2-Z064 & LEU09-OP2-Z145 & \\
\hline LEU09-OP2-Z142 & & LEU09-OP2-Z065 & LEU09-OP2-Z146 & \\
\hline LEU09-OP2-Z153 & & LEU09-OP2-Z067 & LEU09-OP2-Z147 & \\
\hline LEU09-OP2-Z154 & & LEU09-OP2-Z068 & LEU09-OP2-Z148 & \\
\hline LEU09-OP2-Z156 & & LEU09-OP2-Z070 & LEU09-OP2-Z149 & \\
\hline LEU09-OP2-Z167 & & LEU09-OP2-Z071 & LEU09-OP2-Z150 & \\
\hline
\end{tabular}




\section{Summary of acceptance test results for LEU09-OP2-Z}

At the end of this section is the inspection report form IRF-12A associated with the compact lot LEU09-OP2-Z. This inspection report form also appears in section 7 of this compilation, accompanied by the associated data report forms (DRFs) showing the results of each individual measurement. The inspection report form summarizes the acceptance testing performed according to the product inspection plan AGR-CHAR-PIP-12. The information in this form covers all the property specifications listed in section 3.3 of the AGR-2 Fuel Specification (INL SPC-923, Rev. 3). The compact lot, LEU09-OP2-Z, did not meet all the requirements in section 3.3 of SPC-923, Rev. 3. A nonconformance related to a higher than allowed fraction of exposed uranium was determined by the program to be acceptable for the AGR-2 irradiation test. The exposed uranium was due to cracked TRISO layers in the coated particle composite. These cracks are thought to have occurred at $\mathrm{B} \& \mathrm{~W}$ when particles were removed from the coating furnace using a suction device. The final disposition of this compact lot was to "use as is" for the AGR-2 irradiation test. This disposition was documented on INL NCR-44791.

Table 2-1 is provided for quick reference. It gives the mean values of key variable properties of the compact lot, LEU09-OP2-Z. For standard deviations of the distribution of the measured values see the appropriate IRF or DRF. For discussions on the uncertainty in these values, see the associated data acquisition methods and data report forms.

Table 2-1: Quick reference table for key variable properties of LEU09-OP2-Z.

\begin{tabular}{|l|c|}
\hline \multicolumn{1}{|c|}{ Property } & Mean \\
\hline Mean uranium loading (g U/compact) & 1.251 \\
\hline Compact diameter $(\mathrm{mm})$ & 12.29 \\
\hline Compact length $(\mathrm{mm})$ & 25.14 \\
\hline Compact mass $(\mathrm{g})$ & 6.295 \\
\hline Compact matrix density $\left(\mathrm{g} / \mathrm{cm}^{3}\right)$ & 1.59 \\
\hline Impurity content & Table 2-2 \\
\hline
\end{tabular}

The reported mean impurity levels for the fuel compacts, recorded on IRF-12A and IRF-12B, may be higher than the actual values. This is because the as-reported mean impurity levels do not reflect the fact that some of the measurements were at or below the mass spectrometry measurement threshold, and thus could not be differentiated from zero. For the purpose of the acceptance test, impurity values reported as threshold values (documented in the data report forms with the < symbol) are always assumed to be equal to the maximum possible value. In addition, each time a leach was performed, a blank run was also performed, where all the relevant wet chemistry steps in the leach-burn-leach procedure in AGR-CHAR-DAM-26R1 were performed without a compact present, in order to obtain background values for each analyzed impurity. If a measurable impurity value was obtained in the blank, then that value was subtracted from the measured value in each sample. However, if a threshold value was reported in the blank, then no background subtraction was performed. Table 2-2 shows the possible range for the measured impurities, where the upper limit is the as-reported mean and the lower limit is the possible minimum value calculated by accounting for the fact that values reported as 
threshold values could have been as low as zero. This range reflects the uncertainty in the measured impurity values due to the mass spectrometry measurement thresholds.

Table 2-2: Mean impurity levels for fuel compacts from LEU09-OP2-Z compact lot measured by deconsolidation leach-burn-leach technique.

\begin{tabular}{|c|c|}
\hline Impurity & Measured impurity content $(\boldsymbol{\mu g} / \mathbf{c o m p a c t})$ \\
\hline Iron & $0.51-4.04$ \\
\hline Chromium & $0.46-0.61$ \\
\hline Manganese & $0.000-0.136$ \\
\hline Cobalt & $0.000-1.115$ \\
\hline Nickel & $0.38-0.96$ \\
\hline Calcium & $36.20-39.34$ \\
\hline Aluminum & $29.60-29.60$ \\
\hline Titanium & $2.20-2.81$ \\
\hline Vanadium & $16.94-17.09$ \\
\hline
\end{tabular}

Table 2-3 is also provided for quick reference. It gives the binomial distribution calculated upper limit of the $95 \%$ confidence interval of the defect fraction for key attribute properties of the compact lot LEU09-OP2-Z. In other words, these values are the lowest tolerance limits for which the compact lot would be deemed acceptable at $95 \%$ confidence, based on the particular sample that was measured. Also listed in the table are the actual number of defects observed and the number of particles analyzed. Note that in the case of all but the uranium contamination fraction, zero defects were observed. The defect fractions listed in the table for these cases are limited by the number of particles measured and the actual defect fraction could be much lower.

Table 2-3: Quick reference table for key attribute properties of LEU09-OP2-Z.

\begin{tabular}{|l|c|c|}
\hline \multicolumn{1}{|c|}{ Property } & $\begin{array}{c}\text { Observed Number of Defects/ } \\
\text { Number of Particles Analyzed }\end{array}$ & $\begin{array}{c}\text { 95\% Confidence } \\
\text { Defect Fraction }\end{array}$ \\
\hline \hline Uranium contamination fraction & $3 / 317625$ & $\leq 2.5 \mathrm{E}-5$ \\
\hline Defective SiC coating fraction & $0 / 254100$ & $\leq 1.2 \mathrm{E}-5$ \\
\hline Defective IPyC coating fraction & $0 / 63525$ & $\leq 4.8 \mathrm{E}-5$ \\
\hline Defective OPyC coating fraction & $0 / 3176$ & $\leq 9.5 \mathrm{E}-4$ \\
\hline
\end{tabular}

It is also interesting to note the increase in pyrocarbon anisotropy due to compact heat treatment. The diattenuation of the IPyC increased from $0.0116 \pm 0.0004$ to $0.0155 \pm 0.0016(1.0349 \pm 0.0012$ to $1.0465 \pm 0.0049$ in terms of effective $\mathrm{BAFo}$ ). The diattenuation of the OPyC increased from $0.0088 \pm 0.0004$ to $0.0143 \pm 0.0006(1.0263 \pm 0.0011$ to $1.0429 \pm 0.0019$ in terms of effective BAFo). 
ORNL/TM-2010/017

Inspection Report Form IRF-12A: AGR-2 UCO Fuel Compact Lots

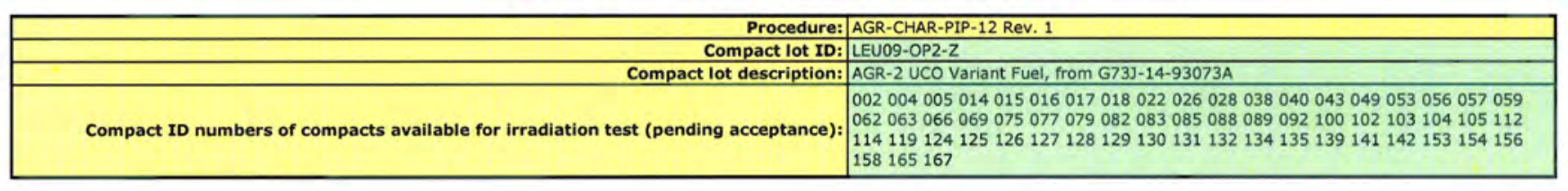

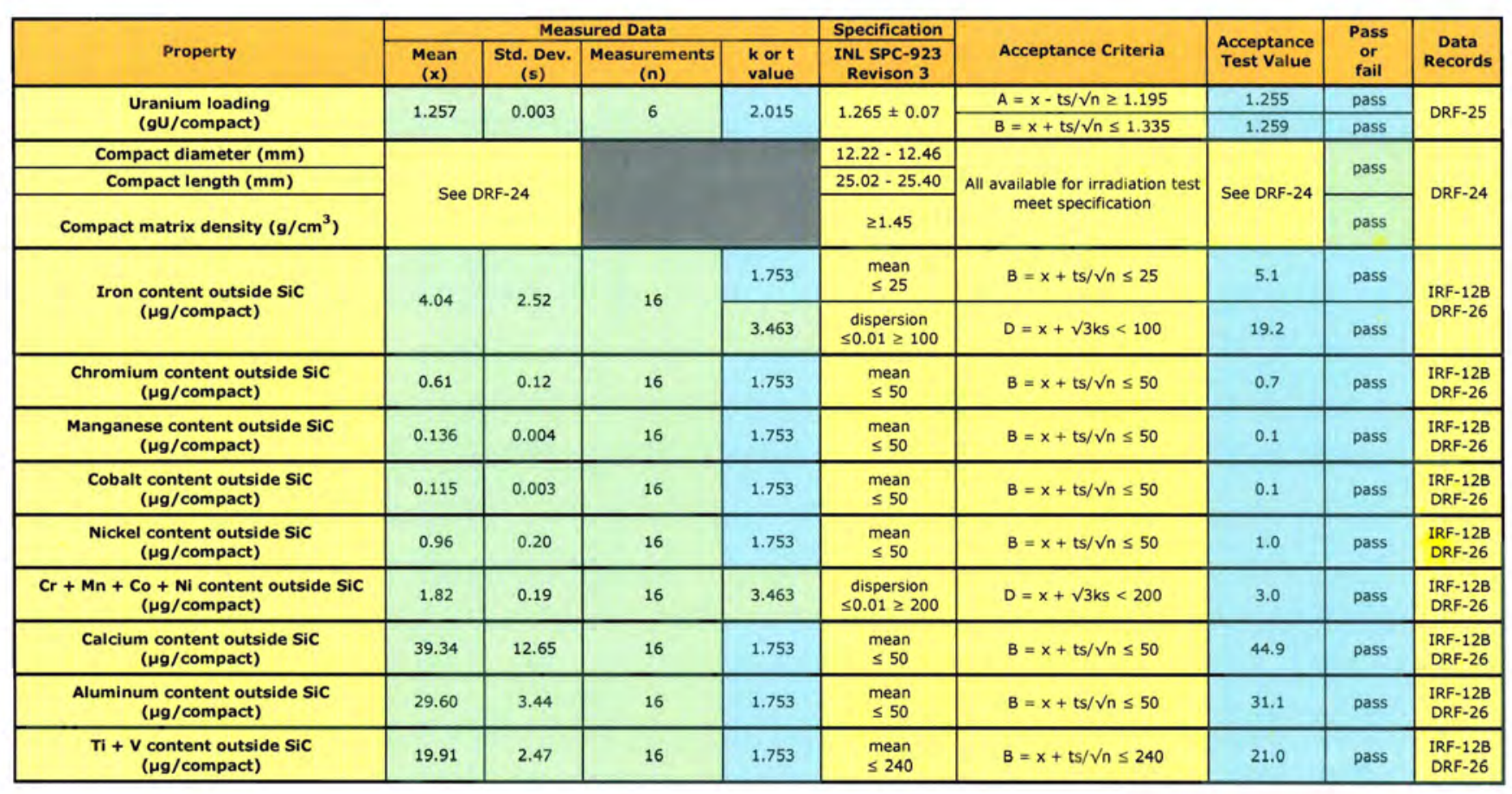

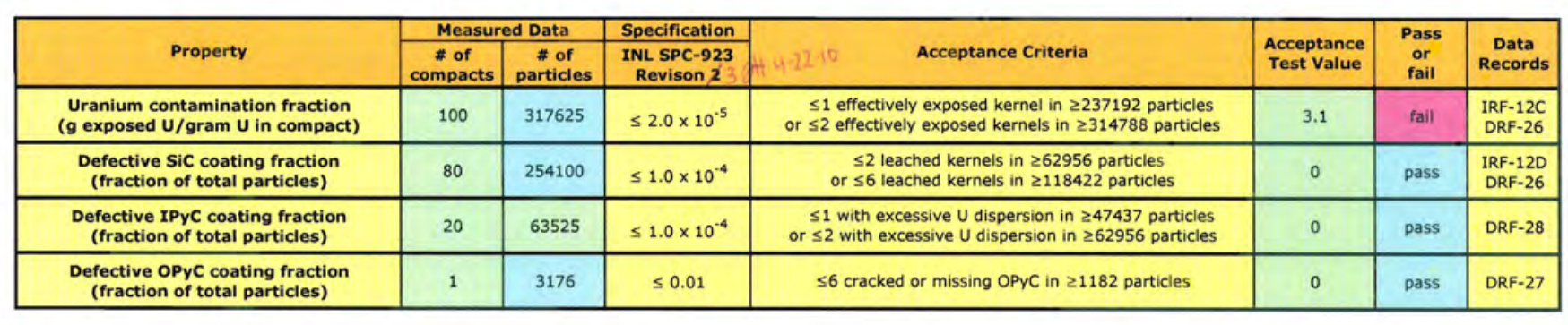

Comments

A $3 / 317625$ uranium contamination fraction is above the specified limit and corresponds to $<2.5 \mathrm{e}-5$ at $95 \%$ confidence. This non-conformance was documented on INL NCR-44791 with a disposition of use as is.

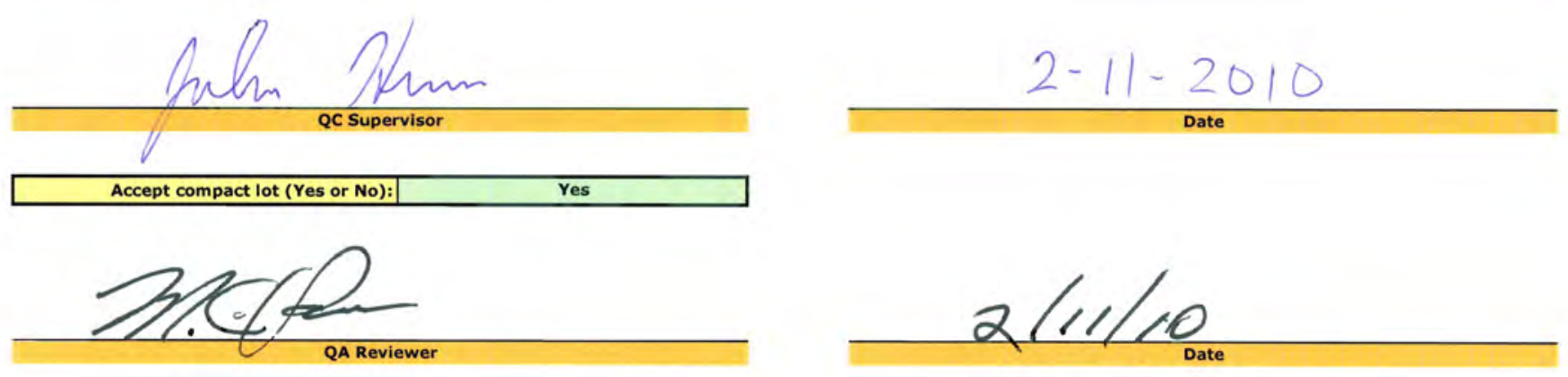

9 


\section{Compacting process conditions}

Four samples totaling $999.9 \mathrm{~g}$ were riffled from coated particle batch G73J-14-93073A using a chute splitter, and shipped from B\&W on April 23, 2009. After receipt, the particles were sorted by size using a roller-micrometer (see Appendix C). No uncoated kernels or coating fragments were found. The first populated bin, corresponding to a roller gap of 750-775 $\mu \mathrm{m}$ and containing the smallest particles, had 14 particles in it. These particles, which were abnormally faceted and non-spherical, were discarded. The remaining material was re-composited and labeled LEU09.

The LEU09 particles were washed in methanol per procedure AGR-TRISOWASH-SOP-1, "Standard Operating Procedure for TRISO Particle Washing." Washing of particles prior to overcoating was adapted in order to help reduce the amount of contamination on the particles that may have been acquired during processing or general handling. This procedure also reduces the amount of loose carbon dust on the surface of the particles. The washing procedure was adopted from General Atomics' particle washing procedures.

After washing, AGR-CHAR-PIP-10, Rev. 2, "Product Inspection Plan for AGR-2 Particles for Compacting - Preliminary Measurements" was completed. This plan calls for measurement of average particle weight, diameter, envelope volume, and uranium content. The plan also calls for riffling of 20 gram aliquots for use as overcoater charges. Riffling at ORNL was done using a 10 position rotary riffler. After riffling out the characterization samples, forty-eight overcoater charges were prepared and labeled LEU09-Y01 through LEU09-Y48. The results of the PIP-10 inspection are reported in section 5. Additional ORNL characterization performed on another sample taken from G73J-14-93073A is provided in ORNL/TM-2008/134, "Data Compilation for AGR-2 UCO Variant Coated Particle Batch G73J-14-93073A."

One $\sim 20 \mathrm{~g}$ aliquot was used per overcoating run. Overcoating was performed according to AGRCOMP-SOP-2, Rev. 1, "Standard Operating Procedure for Overcoating TRISO Particles." The LEU09-Y01 through Y41 riffled aliquots were overcoated with the following matrix batches: Y01-Y17 (RDKrS 050809), Y18-Y34 (RDKrS 052109), and Y35-Y41 (RDKrS 060109). The Y42 to Y48 overcoating charges were not used because a sufficient quantity of correctly sized overcoated particles was accumulated with charges Y01-Y41.

In total, 1265 grams of $-12 /+16$ overcoated particles were produced by overcoating TRISO aliquots Y01-Y41. "-12/+16" overcoated particles are those that pass through an ASTM E11 No. 12 sieve (1.70 mm nominal opening) but do not pass through an ASTM E11 No. 16 sieve (1.18 $\mathrm{mm}$ nominal opening). The 1265 grams of sieved overcoated particles was tabled and 1169 grams of Bin 3 particles were recovered. "Bin 3" particles are those particles that end up in the third bin of a shape separation inclined table; these are the most spherical overcoated particles. 1169 grams of Bin 3 overcoated particles was determined to be a sufficient quantity to produce at least 180 compacts, based on preliminary calculations. The total number of compacts required for acceptance testing, irradiation, and spares was 167. 
After overcoating, the overcoated particles from Y01-Y41 were combined and homogenized into an overcoated particle composite. The overcoated particle composite was labeled LEU09-OP2 and AGR-CHAR-PIP-11, Rev. 0, "Product Inspection Plan for AGR-2 Overcoated Particles for Compacting" was completed. This plan calls for measurement of average overcoated particle weight and diameter. The results of the PIP-11 inspection are reported in section 7. The plan also calls for riffling of compact charges for pressing.

Based on the average uranium loading determined for the LEU09 particles of $3.964 \mathrm{E}-4 \mathrm{~g}$ (section 5), 3191 particles would be needed in each compact to obtain an average uranium loading of $1.265 \mathrm{~g}$ for the compacts (the specified loading in SPC-923 was $1.265 \pm 0.07 \mathrm{~g}$ ). However, for consistency with the LEU06-OP1-Z compact lot (see LEU06-OP1-Z compacts data package ORNL/TM-2009/304) a target of 3200 particles per compact was chosen. This compact charge was based on the measured average uranium loading for LEU06 particles of 3.953E-4 g. Note that both of these particle batches used the same kernel charge so the uranium loading was expected to be the same. The average LEU09-OP2 overcoated particle weight was measured to be $2.010 \mathrm{E}-3 \mathrm{~g}$ (section 6). Using this value, a compact charge of $6.4313 \mathrm{~g}$ was calculated in order to achieve a compact with a uranium loading of $1.265 \pm 0.07 \mathrm{~g}$. One hundred and eighty five compact charges were prepared and labeled LEU09-OP2-G001 through G185. A record of the weight of each compact charge can be found on data report form DRF-24D, in section 7.

Actual compact uranium loading was measured to be $1.257 \pm 0.003 \mathrm{~g}$. Twenty compacts were deconsolidated and the particles were counted as part of the x-ray analysis for possible uranium dispersion due to defective IPyC. The average number of particles per compact was determined to be 3176, 24 particles short of the target compact loading. This reduced number of particles per compact explains the slightly low uranium loading result. It is hypothesized that the reason for the undershoot in particles per compact was due to weight loss from the overcoated particles from evaporation of methanol and volatiles from the resin. Overcoated particles were kept in sealed containers as much as feasible during riffling of the compact charges. However, it is likely that the weight loss due to evaporation for the samples used to determine average particle weight was greater than for the overcoated particles weighed out into each compact charge. Therefore, the average overcoated particle weight used to calculate the target compact charge was slightly too low.

Note that all the uranium per particle measurements on the LEU06, LEU07 and LEU09 material were consistent to within the sampling error and measurement uncertainty, as expected given that the same kernel batch was used for both coating runs. Table 3-1 shows the measured average uranium content in the particles from the uranium analyses performed directly on the particles compared to that calculated from the analyses performed on the compacts divided by the average number of particles per compact.

Table 3-1: Average and standard deviation ( \pm value) of four independent measurements of uranium per particle for LEU06, LEU07 and LEU09 particles.

\begin{tabular}{|l|c|c|c|}
\hline & LEU06 & LEU07 & LEU09 \\
\hline \hline $\begin{array}{l}\text { grams U/particle } \\
\text { based on particle analysis }\end{array}$ & $3.953 \pm 0.010 \cdot 10^{-4} \mathrm{~g}$ & $3.930 \pm 0.007 \cdot 10^{-4} \mathrm{~g}$ & $3.964 \pm 0.009 \cdot 10^{-4} \mathrm{~g}$ \\
\hline
\end{tabular}




\begin{tabular}{|l|l|l|l|}
\hline $\begin{array}{l}\text { grams U/particle } \\
\text { based on compact analysis }\end{array}$ & $3.942 \pm 0.006 \cdot 10^{-4} \mathrm{~g}$ & $3.938 \pm 0.016 \cdot 10^{-4} \mathrm{~g}$ & $3.958 \pm 0.009 \cdot 10^{-4} \mathrm{~g}$ \\
\hline
\end{tabular}

The LEU09-OP2-G\#\# compacting charges were formed into green compacts using a heated, double acting die and a Promess servo-press. Compacting was performed in accordance with AGR-COMP-SOP-3, Rev. 3, "Standard Operating Procedure for Compacting Using a Servo Press." The die was heated to $65^{\circ} \mathrm{C}$ and approximately $0.10 \mathrm{~g}$ of matrix was added to the top and bottom of the compact in order to create matrix "end caps." The end caps were formed with the compact by first pouring a matrix charge into the heated die, followed by the overcoated particles, and then a second charge of matrix. This forming method created a thin (less than 0.5 mm thick) fuel free zone on the ends of the compact, called end caps. In total, 185 green compacts were fabricated. The compacts retained the designation of the riffled charges, LEU09OP2-G001 through G185. All 185 green compacts were carbonized and heat treated according to AGR-COMP-SOP-4, Rev. 0, "Standard Operating Procedure for Carbonizing Compacts," and AGR-COMP-SOP-5 Rev. 1, "Standard Operating Procedure for Heat-treating Compacts."

A significant change was made to the compacting equipment from the LEU06 and LEU07 campaigns to the LEU08, LEU09, and LEU11 campaigns. The Carver hydraulic press was replaced with a Promess servo-press. The Promess press provided pressing rate and piston displacement control to three decimal place accuracy. This enabled the pressing rate and compact length to be precisely set and repeated for each individual LEU09-OP2-G\#\#\# compacting charge. The force for each compact was also recorded more accurately, to \pm 2 lbs.-f. The switch from the Carver press to the Promess press is the reason the compacting procedure was rewritten and a new revision was issued.

After compacting, 167 compacts were selected from LEU09-OP2-G001 through G185 for use. Compacts with obvious processing defects, chips, or undesirable dimensions were sorted out and not included in the 167 compacts selected for the final fuel compact lot. This down-select was part of the compacting process and was performed prior to random selection of compacts for acceptance testing. It should be understood that the results in this section and the acceptance testing are only relevant for the final 167 compact lot from which random representative samples were drawn for characterization. As instructed in AGR-CHAR-PIP-12, Rev. 1, "Product Inspection Plan for AGR-2 UCO Fuel Compact Lots," these 167 compacts were randomized and relabeled as LEU09-OP2-Z001 through Z167. A record of the original G-number for each Znumbered compact can be found on data report form DRF-24C, in section 7. After relabeling, the compacts were characterized for product acceptance according to product inspection plan PIP12. This plan calls for measurement of compact length, diameter, mass, matrix density, uranium content, impurity content, and determination of defect fractions for exposed uranium, defective $\mathrm{SiC}$, uranium dispersion due to defective IPyC, and defective OPyC. 


\section{AGR-2 Process Conditions}

The LEU09-OP2-Z (AGR-2 UCO Variant) compact lot was made in accordance with the AGR-2 Fuel Specification (SPC-923, Rev. 3). The specified AGR-2 process limits are listed below.

Molding Pressure: $\quad<60 \mathrm{MPa}$

Carbonization parameters: $\quad<350^{\circ} \mathrm{C} / \mathrm{hr}$ in $\mathrm{He}$ atmosphere

Hold at $950 \pm 50^{\circ} \mathrm{C}$ for $1.0 \pm 0.4 \mathrm{hr}$ Furnace cool

Heat treatment parameters: $\quad 20^{\circ} \mathrm{C} / \mathrm{min}$ in vacuum $(<1.3 \mathrm{~Pa})$

Hold at $1650-1850^{\circ} \mathrm{C}$ for $60 \pm 10 \mathrm{~min}$

Furnace cool at $20^{\circ} \mathrm{C} / \mathrm{min}$ to below $700^{\circ} \mathrm{C}$

Table 3-2 shows the process conditions used in molding the compacts, carbonizing the compacts, and heat treating the compacts. In the carbonization regime, the furnace was allowed to cool under no power (i.e., after holding at $950^{\circ} \mathrm{C}$ for 1 hour, power was turned off). In the heat treatment run, the furnace was cooled under power at $20^{\circ} \mathrm{C} / \mathrm{min}$ until the furnace temperature reached $700^{\circ} \mathrm{C}$, and then the furnace was allowed to cool under no power. 
Table 3-2: Summary of process conditions used in making LEU09-OP2-Z (AGR-2 UCO Variant) compacts

\begin{tabular}{|c|c|c|c|c|c|c|c|c|c|}
\hline \multirow{2}{*}{ Compact ID } & \multicolumn{5}{|c|}{ Carbonization Parameter } & \multicolumn{4}{|c|}{ Heat-treatment Parameters } \\
\hline & $\begin{array}{l}\text { Molding Pressure } \\
\qquad(\mathrm{MPa})\end{array}$ & $\begin{array}{l}\text { Heating Rate } \\
\left({ }^{\circ} \mathrm{C} / \mathrm{min} .\right)\end{array}$ & $\begin{array}{l}\text { Max. Temp. } \\
\left({ }^{\circ} \mathrm{C}\right)\end{array}$ & $\begin{array}{l}\text { Hold Time } \\
\quad \text { (hrs.) }\end{array}$ & Atmosphere & $\begin{array}{l}\text { Heating Rate } \\
\left({ }^{\circ} \mathrm{C} / \mathrm{min} .\right)\end{array}$ & $\begin{array}{l}\text { Max. Temp. } \\
\left({ }^{\circ} \mathrm{C}\right)\end{array}$ & $\begin{array}{l}\text { Hold Time } \\
\text { (hrs.) }\end{array}$ & Atmosphere \\
\hline LEU09-OP2-Z001 & 16.8 & 4.7 & 950 & 1 & flowing $\mathrm{He}$ & 20 & 1800 & 1 & vacuum \\
\hline LEU09-OP2-Z002 & 16.0 & 4.7 & 950 & 1 & flowing $\mathrm{He}$ & 20 & 1800 & 1 & vacuum \\
\hline LEU09-OP2-Z003 & 16.4 & 4.7 & 950 & 1 & flowing $\mathrm{He}$ & 20 & 1800 & 1 & vacuum \\
\hline LEU09-OP2-Z004 & 17.0 & 4.7 & 950 & 1 & flowing $\mathrm{He}$ & 20 & 1800 & 1 & vacuum \\
\hline LEU09-OP2-Z005 & 17.2 & 4.7 & 950 & 1 & flowing $\mathrm{He}$ & 20 & 1800 & 1 & vacuum \\
\hline LEU09-OP2-Z006 & 16.1 & 4.7 & 950 & 1 & flowing $\mathrm{He}$ & 20 & 1800 & 1 & vacuum \\
\hline LEU09-OP2-Z007 & 16.8 & 4.7 & 950 & 1 & flowing $\mathrm{He}$ & 20 & 1800 & 1 & vacuum \\
\hline LEU09-OP2-Z008 & 16.7 & 4.7 & 950 & 1 & flowing $\mathrm{He}$ & 20 & 1800 & 1 & vacuum \\
\hline LEU09-OP2-Z009 & 16.1 & 4.7 & 950 & 1 & flowing $\mathrm{He}$ & 20 & 1800 & 1 & vacuum \\
\hline LEU09-OP2-Z010 & 16.8 & 4.7 & 950 & 1 & flowing $\mathrm{He}$ & 20 & 1800 & 1 & vacuum \\
\hline LEU09-OP2-Z011 & 16.9 & 4.7 & 950 & 1 & flowing $\mathrm{He}$ & 20 & 1800 & 1 & vacuum \\
\hline LEU09-OP2-Z012 & 17.2 & 4.7 & 950 & 1 & flowing $\mathrm{He}$ & 20 & 1800 & 1 & vacuum \\
\hline LEU09-OP2-Z013 & 16.8 & 4.7 & 950 & 1 & flowing $\mathrm{He}$ & 20 & 1800 & 1 & vacuum \\
\hline LEU09-OP2-Z014 & 16.1 & 4.7 & 950 & 1 & flowing $\mathrm{He}$ & 20 & 1800 & 1 & vacuum \\
\hline LEU09-OP2-Z015 & 16.0 & 4.7 & 950 & 1 & flowing $\mathrm{He}$ & 20 & 1800 & 1 & vacuum \\
\hline LEU09-OP2-Z016 & 16.4 & 4.7 & 950 & 1 & flowing $\mathrm{He}$ & 20 & 1800 & 1 & vacuum \\
\hline LEU09-OP2-Z017 & 15.7 & 4.7 & 950 & 1 & flowing $\mathrm{He}$ & 20 & 1800 & 1 & vacuum \\
\hline LEU09-OP2-Z018 & 16.3 & 4.7 & 950 & 1 & flowing $\mathrm{He}$ & 20 & 1800 & 1 & vacuum \\
\hline LEU09-OP2-Z019 & 18.2 & 4.7 & 950 & 1 & flowing $\mathrm{He}$ & 20 & 1800 & 1 & vacuum \\
\hline LEU09-OP2-Z020 & 17.2 & 4.7 & 950 & 1 & flowing $\mathrm{He}$ & 20 & 1800 & 1 & vacuum \\
\hline LEU09-OP2-Z021 & 17.7 & 4.7 & 950 & 1 & flowing $\mathrm{He}$ & 20 & 1800 & 1 & vacuum \\
\hline LEU09-OP2-Z022 & 16.8 & 4.7 & 950 & 1 & flowing $\mathrm{He}$ & 20 & 1800 & 1 & vacuum \\
\hline LEU09-OP2-Z023 & 17.0 & 4.7 & 950 & 1 & flowing $\mathrm{He}$ & 20 & 1800 & 1 & vacuum \\
\hline LEU09-OP2-Z024 & 16.8 & 4.7 & 950 & 1 & flowing $\mathrm{He}$ & 20 & 1800 & 1 & vacuum \\
\hline LEU09-OP2-Z025 & 16.1 & 4.7 & 950 & 1 & flowing $\mathrm{He}$ & 20 & 1800 & 1 & vacuum \\
\hline LEU09-OP2-Z026 & 17.0 & 4.7 & 950 & 1 & flowing $\mathrm{He}$ & 20 & 1800 & 1 & vacuum \\
\hline LEU09-OP2-Z027 & 16.6 & 4.7 & 950 & 1 & flowing $\mathrm{He}$ & 20 & 1800 & 1 & vacuum \\
\hline LEU09-OP2-Z028 & 16.8 & 4.7 & 950 & 1 & flowing $\mathrm{He}$ & 20 & 1800 & 1 & vacuum \\
\hline LEU09-OP2-Z029 & 16.9 & 4.7 & 950 & 1 & flowing $\mathrm{He}$ & 20 & 1800 & 1 & vacuum \\
\hline LEU09-OP2-Z030 & 16.9 & 4.7 & 950 & 1 & flowing $\mathrm{He}$ & 20 & 1800 & 1 & vacuum \\
\hline
\end{tabular}


Table 3-2 (cont.): Summary of process conditions used in making LEU09-OP2-Z (AGR-2 UCO Variant) compacts

\begin{tabular}{|c|c|c|c|c|c|c|c|c|c|}
\hline \multirow{2}{*}{ Compact ID } & \multicolumn{5}{|c|}{ Carbonization Parameter } & \multicolumn{4}{|c|}{ Heat-treatment Parameters } \\
\hline & $\begin{array}{l}\text { Molding Pressure } \\
(\mathrm{MPa})\end{array}$ & $\begin{array}{l}\text { Heating Rate } \\
\left({ }^{\circ} \mathrm{C} / \mathrm{min} .\right)\end{array}$ & $\begin{array}{l}\text { Max. Temp. } \\
\left({ }^{\circ} \mathrm{C}\right)\end{array}$ & $\begin{array}{l}\text { Hold Time } \\
\text { (hrs.) }\end{array}$ & Atmosphere & $\begin{array}{l}\text { Heating Rate } \\
\left({ }^{\circ} \mathrm{C} / \mathrm{min} .\right)\end{array}$ & $\begin{array}{l}\text { Max. Temp. } \\
\left({ }^{\circ} \mathrm{C}\right)\end{array}$ & $\begin{array}{l}\text { Hold Time } \\
\text { (hrs.) }\end{array}$ & Atmosphere \\
\hline LEU09-OP2-Z031 & 16.6 & 4.7 & 950 & 1 & flowing $\mathrm{He}$ & 20 & 1800 & 1 & vacuum \\
\hline LEU09-OP2-Z032 & 16.6 & 4.7 & 950 & 1 & flowing $\mathrm{He}$ & 20 & 1800 & 1 & vacuum \\
\hline LEU09-OP2-Z033 & 17.0 & 4.7 & 950 & 1 & flowing $\mathrm{He}$ & 20 & 1800 & 1 & vacuum \\
\hline LEU09-OP2-Z034 & 16.4 & 4.7 & 950 & 1 & flowing $\mathrm{He}$ & 20 & 1800 & 1 & vacuum \\
\hline LEU09-OP2-Z035 & 16.4 & 4.7 & 950 & 1 & flowing $\mathrm{He}$ & 20 & 1800 & 1 & vacuum \\
\hline LEU09-OP2-Z036 & 17.4 & 4.7 & 950 & 1 & flowing $\mathrm{He}$ & 20 & 1800 & 1 & vacuum \\
\hline LEU09-OP2-Z037 & 16.4 & 4.7 & 950 & 1 & flowing $\mathrm{He}$ & 20 & 1800 & 1 & vacuum \\
\hline LEU09-OP2-Z038 & 15.8 & 4.7 & 950 & 1 & flowing $\mathrm{He}$ & 20 & 1800 & 1 & vacuum \\
\hline LEU09-OP2-Z039 & 17.2 & 4.7 & 950 & 1 & flowing $\mathrm{He}$ & 20 & 1800 & 1 & vacuum \\
\hline LEU09-OP2-Z040 & 17.0 & 4.7 & 950 & 1 & flowing $\mathrm{He}$ & 20 & 1800 & 1 & vacuum \\
\hline LEU09-OP2-Z041 & 17.6 & 4.7 & 950 & 1 & flowing $\mathrm{He}$ & 20 & 1800 & 1 & vacuum \\
\hline LEU09-OP2-Z042 & 16.9 & 4.7 & 950 & 1 & flowing $\mathrm{He}$ & 20 & 1800 & 1 & vacuum \\
\hline LEU09-OP2-Z043 & 17.1 & 4.7 & 950 & 1 & flowing $\mathrm{He}$ & 20 & 1800 & 1 & vacuum \\
\hline LEU09-OP2-Z044 & 17.9 & 4.7 & 950 & 1 & flowing $\mathrm{He}$ & 20 & 1800 & 1 & vacuum \\
\hline LEU09-OP2-Z045 & 16.4 & 4.7 & 950 & 1 & flowing $\mathrm{He}$ & 20 & 1800 & 1 & vacuum \\
\hline LEU09-OP2-Z046 & 16.7 & 4.7 & 950 & 1 & flowing $\mathrm{He}$ & 20 & 1800 & 1 & vacuum \\
\hline LEU09-OP2-Z047 & 16.7 & 4.7 & 950 & 1 & flowing $\mathrm{He}$ & 20 & 1800 & 1 & vacuum \\
\hline LEU09-OP2-Z048 & 17.4 & 4.7 & 950 & 1 & flowing $\mathrm{He}$ & 20 & 1800 & 1 & vacuum \\
\hline LEU09-OP2-Z049 & 16.3 & 4.7 & 950 & 1 & flowing $\mathrm{He}$ & 20 & 1800 & 1 & vacuum \\
\hline LEU09-OP2-Z050 & 16.8 & 4.7 & 950 & 1 & flowing $\mathrm{He}$ & 20 & 1800 & 1 & vacuum \\
\hline LEU09-OP2-Z051 & 17.3 & 4.7 & 950 & 1 & flowing $\mathrm{He}$ & 20 & 1800 & 1 & vacuum \\
\hline LEU09-OP2-Z052 & 17.4 & 4.7 & 950 & 1 & flowing $\mathrm{He}$ & 20 & 1800 & 1 & vacuum \\
\hline LEU09-OP2-Z053 & 16.4 & 4.7 & 950 & 1 & flowing $\mathrm{He}$ & 20 & 1800 & 1 & vacuum \\
\hline LEU09-OP2-Z054 & 17.0 & 4.7 & 950 & 1 & flowing $\mathrm{He}$ & 20 & 1800 & 1 & vacuum \\
\hline LEU09-OP2-Z055 & 16.7 & 4.7 & 950 & 1 & flowing $\mathrm{He}$ & 20 & 1800 & 1 & vacuum \\
\hline LEU09-OP2-Z056 & 17.2 & 4.7 & 950 & 1 & flowing $\mathrm{He}$ & 20 & 1800 & 1 & vacuum \\
\hline LEU09-OP2-Z057 & 16.9 & 4.7 & 950 & 1 & flowing $\mathrm{He}$ & 20 & 1800 & 1 & vacuum \\
\hline LEU09-OP2-Z058 & 16.3 & 4.7 & 950 & 1 & flowing $\mathrm{He}$ & 20 & 1800 & 1 & vacuum \\
\hline LEU09-OP2-Z059 & 16.9 & 4.7 & 950 & 1 & flowing $\mathrm{He}$ & 20 & 1800 & 1 & vacuum \\
\hline LEU09-OP2-Z060 & 16.7 & 4.7 & 950 & 1 & flowing $\mathrm{He}$ & 20 & 1800 & 1 & vacuum \\
\hline
\end{tabular}


Table 3-2 (cont.): Summary of process conditions used in making LEU09-OP2-Z (AGR-2 UCO Variant) compacts

\begin{tabular}{|c|c|c|c|c|c|c|c|c|c|}
\hline \multirow{2}{*}{ Compact ID } & \multicolumn{5}{|c|}{ Carbonization Parameter } & \multicolumn{4}{|c|}{ Heat-treatment Parameters } \\
\hline & $\begin{array}{l}\text { Molding Pressure } \\
(\mathrm{MPa})\end{array}$ & $\begin{array}{l}\text { Heating Rate } \\
\left({ }^{\circ} \mathrm{C} / \mathrm{min} .\right)\end{array}$ & $\begin{array}{l}\text { Max. Temp. } \\
\left({ }^{\circ} \mathrm{C}\right)\end{array}$ & $\begin{array}{l}\text { Hold Time } \\
\text { (hrs.) }\end{array}$ & Atmosphere & $\begin{array}{l}\text { Heating Rate } \\
\left({ }^{\circ} \mathrm{C} / \mathrm{min} .\right)\end{array}$ & $\begin{array}{l}\text { Max. Temp. } \\
\left({ }^{\circ} \mathrm{C}\right)\end{array}$ & $\begin{array}{l}\text { Hold Time } \\
\text { (hrs.) }\end{array}$ & Atmosphere \\
\hline LEU09-OP2-Z061 & 17.1 & 4.7 & 950 & 1 & flowing $\mathrm{He}$ & 20 & 1800 & 1 & vacuum \\
\hline LEU09-OP2-Z062 & 16.2 & 4.7 & 950 & 1 & flowing $\mathrm{He}$ & 20 & 1800 & 1 & vacuum \\
\hline LEU09-OP2-Z063 & 16.8 & 4.7 & 950 & 1 & flowing $\mathrm{He}$ & 20 & 1800 & 1 & vacuum \\
\hline LEU09-OP2-Z064 & 16.7 & 4.7 & 950 & 1 & flowing $\mathrm{He}$ & 20 & 1800 & 1 & vacuum \\
\hline LEU09-OP2-Z065 & 17.0 & 4.7 & 950 & 1 & flowing $\mathrm{He}$ & 20 & 1800 & 1 & vacuum \\
\hline LEU09-OP2-Z066 & 16.9 & 4.7 & 950 & 1 & flowing $\mathrm{He}$ & 20 & 1800 & 1 & vacuum \\
\hline LEU09-OP2-Z067 & 16.7 & 4.7 & 950 & 1 & flowing $\mathrm{He}$ & 20 & 1800 & 1 & vacuum \\
\hline LEU09-OP2-Z068 & 17.2 & 4.7 & 950 & 1 & flowing $\mathrm{He}$ & 20 & 1800 & 1 & vacuum \\
\hline LEU09-OP2-Z069 & 17.6 & 4.7 & 950 & 1 & flowing $\mathrm{He}$ & 20 & 1800 & 1 & vacuum \\
\hline LEU09-OP2-Z070 & 16.3 & 4.7 & 950 & 1 & flowing $\mathrm{He}$ & 20 & 1800 & 1 & vacuum \\
\hline LEU09-OP2-Z071 & 17.1 & 4.7 & 950 & 1 & flowing $\mathrm{He}$ & 20 & 1800 & 1 & vacuum \\
\hline LEU09-OP2-Z072 & 17.8 & 4.7 & 950 & 1 & flowing $\mathrm{He}$ & 20 & 1800 & 1 & vacuum \\
\hline LEU09-OP2-Z073 & 16.5 & 4.7 & 950 & 1 & flowing $\mathrm{He}$ & 20 & 1800 & 1 & vacuum \\
\hline LEU09-OP2-Z074 & 16.6 & 4.7 & 950 & 1 & flowing He & 20 & 1800 & 1 & vacuum \\
\hline LEU09-OP2-Z075 & 17.7 & 4.7 & 950 & 1 & flowing $\mathrm{He}$ & 20 & 1800 & 1 & vacuum \\
\hline LEU09-OP2-Z076 & 17.4 & 4.7 & 950 & 1 & flowing $\mathrm{He}$ & 20 & 1800 & 1 & vacuum \\
\hline LEU09-OP2-Z077 & 16.9 & 4.7 & 950 & 1 & flowing $\mathrm{He}$ & 20 & 1800 & 1 & vacuum \\
\hline LEU09-OP2-Z078 & 16.7 & 4.7 & 950 & 1 & flowing $\mathrm{He}$ & 20 & 1800 & 1 & vacuum \\
\hline LEU09-OP2-Z079 & 16.7 & 4.7 & 950 & 1 & flowing $\mathrm{He}$ & 20 & 1800 & 1 & vacuum \\
\hline LEU09-OP2-Z080 & 16.1 & 4.7 & 950 & 1 & flowing $\mathrm{He}$ & 20 & 1800 & 1 & vacuum \\
\hline LEU09-OP2-Z081 & 17.8 & 4.7 & 950 & 1 & flowing $\mathrm{He}$ & 20 & 1800 & 1 & vacuum \\
\hline LEU09-OP2-Z082 & 17.5 & 4.7 & 950 & 1 & flowing $\mathrm{He}$ & 20 & 1800 & 1 & vacuum \\
\hline LEU09-OP2-Z083 & 16.7 & 4.7 & 950 & 1 & flowing $\mathrm{He}$ & 20 & 1800 & 1 & vacuum \\
\hline LEU09-OP2-Z084 & 17.1 & 4.7 & 950 & 1 & flowing $\mathrm{He}$ & 20 & 1800 & 1 & vacuum \\
\hline LEU09-OP2-Z085 & 16.5 & 4.7 & 950 & 1 & flowing $\mathrm{He}$ & 20 & 1800 & 1 & vacuum \\
\hline LEU09-OP2-Z086 & 16.5 & 4.7 & 950 & 1 & flowing $\mathrm{He}$ & 20 & 1800 & 1 & vacuum \\
\hline LEU09-OP2-Z087 & 17.2 & 4.7 & 950 & 1 & flowing $\mathrm{He}$ & 20 & 1800 & 1 & vacuum \\
\hline LEU09-OP2-Z088 & 17.0 & 4.7 & 950 & 1 & flowing $\mathrm{He}$ & 20 & 1800 & 1 & vacuum \\
\hline LEU09-OP2-Z089 & 16.8 & 4.7 & 950 & 1 & flowing $\mathrm{He}$ & 20 & 1800 & 1 & vacuum \\
\hline LEU09-OP2-Z090 & 16.1 & 4.7 & 950 & 1 & flowing $\mathrm{He}$ & 20 & 1800 & 1 & vacuum \\
\hline \multicolumn{6}{|c|}{ Task Manager Review } & Date & \multicolumn{2}{|c|}{$2-4-10$} & \\
\hline
\end{tabular}


Table 3-2 (cont.): Summary of process conditions used in making LEU09-OP2-Z (AGR-2 UCO Variant) compacts

\begin{tabular}{|c|c|c|c|c|c|c|c|c|c|}
\hline \multirow{2}{*}{ Compact ID } & \multicolumn{5}{|c|}{ Carbonization Parameter } & \multicolumn{4}{|c|}{ Heat-treatment Parameters } \\
\hline & $\begin{array}{l}\text { Molding Pressure } \\
(\mathrm{MPa})\end{array}$ & $\begin{array}{l}\text { Heating Rate } \\
\left({ }^{\circ} \mathrm{C} / \mathrm{min} .\right)\end{array}$ & $\begin{array}{l}\text { Max. Temp. } \\
\left({ }^{\circ} \mathrm{C}\right)\end{array}$ & $\begin{array}{l}\text { Hold Time } \\
\text { (hrs.) }\end{array}$ & Atmosphere & $\begin{array}{l}\text { Heating Rate } \\
\left({ }^{\circ} \mathrm{C} / \mathrm{min} .\right)\end{array}$ & $\begin{array}{l}\text { Max. Temp. } \\
\left({ }^{\circ} \mathrm{C}\right)\end{array}$ & $\begin{array}{l}\text { Hold Time } \\
\text { (hrs.) }\end{array}$ & Atmosphere \\
\hline LEU09-OP2-Z091 & 16.7 & 4.7 & 950 & 1 & flowing $\mathrm{He}$ & 20 & 1800 & 1 & vacuum \\
\hline LEU09-OP2-Z092 & 17.3 & 4.7 & 950 & 1 & flowing $\mathrm{He}$ & 20 & 1800 & 1 & vacuum \\
\hline LEU09-OP2-Z093 & 16.8 & 4.7 & 950 & 1 & flowing $\mathrm{He}$ & 20 & 1800 & 1 & vacuum \\
\hline LEU09-OP2-Z094 & 16.0 & 4.7 & 950 & 1 & flowing $\mathrm{He}$ & 20 & 1800 & 1 & vacuum \\
\hline LEU09-OP2-Z095 & 16.4 & 4.7 & 950 & 1 & flowing $\mathrm{He}$ & 20 & 1800 & 1 & vacuum \\
\hline LEU09-OP2-Z096 & 17.0 & 4.7 & 950 & 1 & flowing $\mathrm{He}$ & 20 & 1800 & 1 & vacuum \\
\hline LEU09-OP2-Z097 & 18.0 & 4.7 & 950 & 1 & flowing $\mathrm{He}$ & 20 & 1800 & 1 & vacuum \\
\hline LEU09-OP2-Z098 & 16.3 & 4.7 & 950 & 1 & flowing $\mathrm{He}$ & 20 & 1800 & 1 & vacuum \\
\hline LEU09-OP2-Z099 & 17.1 & 4.7 & 950 & 1 & flowing $\mathrm{He}$ & 20 & 1800 & 1 & vacuum \\
\hline LEU09-OP2-Z100 & 16.3 & 4.7 & 950 & 1 & flowing $\mathrm{He}$ & 20 & 1800 & 1 & vacuum \\
\hline LEU09-OP2-Z101 & 17.9 & 4.7 & 950 & 1 & flowing $\mathrm{He}$ & 20 & 1800 & 1 & vacuum \\
\hline LEU09-OP2-Z102 & 17.0 & 4.7 & 950 & 1 & flowing $\mathrm{He}$ & 20 & 1800 & 1 & vacuum \\
\hline LEU09-OP2-Z103 & 16.6 & 4.7 & 950 & 1 & flowing $\mathrm{He}$ & 20 & 1800 & 1 & vacuum \\
\hline LEU09-OP2-Z104 & 17.8 & 4.7 & 950 & 1 & flowing $\mathrm{He}$ & 20 & 1800 & 1 & vacuum \\
\hline LEU09-OP2-Z105 & 17.0 & 4.7 & 950 & 1 & flowing $\mathrm{He}$ & 20 & 1800 & 1 & vacuum \\
\hline LEU09-OP2-Z106 & 16.6 & 4.7 & 950 & 1 & flowing $\mathrm{He}$ & 20 & 1800 & 1 & vacuum \\
\hline LEU09-OP2-Z107 & 16.6 & 4.7 & 950 & 1 & flowing He & 20 & 1800 & 1 & vacuum \\
\hline LEU09-OP2-Z108 & 17.0 & 4.7 & 950 & 1 & flowing $\mathrm{He}$ & 20 & 1800 & 1 & vacuum \\
\hline LEU09-OP2-Z109 & 16.9 & 4.7 & 950 & 1 & flowing $\mathrm{He}$ & 20 & 1800 & 1 & vacuum \\
\hline LEU09-OP2-Z110 & 16.6 & 4.7 & 950 & 1 & flowing $\mathrm{He}$ & 20 & 1800 & 1 & vacuum \\
\hline LEU09-OP2-Z111 & 16.8 & 4.7 & 950 & 1 & flowing $\mathrm{He}$ & 20 & 1800 & 1 & vacuum \\
\hline LEU09-OP2-Z112 & 16.2 & 4.7 & 950 & 1 & flowing $\mathrm{He}$ & 20 & 1800 & 1 & vacuum \\
\hline LEU09-OP2-Z113 & 16.4 & 4.7 & 950 & 1 & flowing $\mathrm{He}$ & 20 & 1800 & 1 & vacuum \\
\hline LEU09-OP2-Z114 & 16.9 & 4.7 & 950 & 1 & flowing $\mathrm{He}$ & 20 & 1800 & 1 & vacuum \\
\hline LEU09-OP2-Z115 & 16.8 & 4.7 & 950 & 1 & flowing $\mathrm{He}$ & 20 & 1800 & 1 & vacuum \\
\hline LEU09-OP2-Z116 & 16.7 & 4.7 & 950 & 1 & flowing $\mathrm{He}$ & 20 & 1800 & 1 & vacuum \\
\hline LEU09-OP2-Z117 & 17.4 & 4.7 & 950 & 1 & flowing $\mathrm{He}$ & 20 & 1800 & 1 & vacuum \\
\hline LEU09-OP2-Z118 & 16.9 & 4.7 & 950 & 1 & flowing $\mathrm{He}$ & 20 & 1800 & 1 & vacuum \\
\hline LEU09-OP2-Z119 & 17.1 & 4.7 & 950 & 1 & flowing $\mathrm{He}$ & 20 & 1800 & 1 & vacuum \\
\hline LEU09-OP2-Z120 & 17.5 & 4.7 & 950 & 1 & flowing $\mathrm{He}$ & 20 & 1800 & 1 & vacuum \\
\hline
\end{tabular}


Table 3-2 (cont.): Summary of process conditions used in making LEU09-OP2-Z (AGR-2 UCO Variant) compacts

\begin{tabular}{|c|c|c|c|c|c|c|c|c|c|}
\hline \multirow{2}{*}{ Compact ID } & \multicolumn{5}{|c|}{ Carbonization Parameter } & \multicolumn{4}{|c|}{ Heat-treatment Parameters } \\
\hline & $\begin{array}{l}\text { Molding Pressure } \\
\qquad(\mathrm{MPa})\end{array}$ & $\begin{array}{l}\text { Heating Rate } \\
\left({ }^{\circ} \mathrm{C} / \mathrm{min} .\right)\end{array}$ & $\begin{array}{l}\text { Max. Temp. } \\
\left({ }^{\circ} \mathrm{C}\right)\end{array}$ & $\begin{array}{l}\text { Hold Time } \\
\text { (hrs.) }\end{array}$ & Atmosphere & $\begin{array}{c}\text { Heating Rate } \\
\left({ }^{\circ} \mathrm{C} / \mathrm{min} .\right)\end{array}$ & $\begin{array}{l}\text { Max. Temp. } \\
\left({ }^{\circ} \mathrm{C}\right)\end{array}$ & $\begin{array}{l}\text { Hold Time } \\
\text { (hrs.) }\end{array}$ & Atmosphere \\
\hline LEU09-OP2-Z121 & 16.9 & 4.7 & 950 & 1 & flowing $\mathrm{He}$ & 20 & 1800 & 1 & vacuum \\
\hline LEU09-OP2-Z122 & 16.7 & 4.7 & 950 & 1 & flowing $\mathrm{He}$ & 20 & 1800 & 1 & vacuum \\
\hline LEU09-OP2-Z123 & 16.9 & 4.7 & 950 & 1 & flowing $\mathrm{He}$ & 20 & 1800 & 1 & vacuum \\
\hline LEU09-OP2-Z124 & 16.1 & 4.7 & 950 & 1 & flowing He & 20 & 1800 & 1 & vacuum \\
\hline LEU09-OP2-Z125 & 17.3 & 4.7 & 950 & 1 & flowing $\mathrm{He}$ & 20 & 1800 & 1 & vacuum \\
\hline LEU09-OP2-Z126 & 16.7 & 4.7 & 950 & 1 & flowing $\mathrm{He}$ & 20 & 1800 & 1 & vacuum \\
\hline LEU09-OP2-Z127 & 16.3 & 4.7 & 950 & 1 & flowing $\mathrm{He}$ & 20 & 1800 & 1 & vacuum \\
\hline LEU09-OP2-Z128 & 17.1 & 4.7 & 950 & 1 & flowing $\mathrm{He}$ & 20 & 1800 & 1 & vacuum \\
\hline LEU09-OP2-Z129 & 17.1 & 4.7 & 950 & 1 & flowing $\mathrm{He}$ & 20 & 1800 & 1 & vacuum \\
\hline LEU09-OP2-Z130 & 16.5 & 4.7 & 950 & 1 & flowing $\mathrm{He}$ & 20 & 1800 & 1 & vacuum \\
\hline LEU09-OP2-Z131 & 16.5 & 4.7 & 950 & 1 & flowing $\mathrm{He}$ & 20 & 1800 & 1 & vacuum \\
\hline LEU09-OP2-Z132 & 17.9 & 4.7 & 950 & 1 & flowing $\mathrm{He}$ & 20 & 1800 & 1 & vacuum \\
\hline LEU09-OP2-Z133 & 16.8 & 4.7 & 950 & 1 & flowing $\mathrm{He}$ & 20 & 1800 & 1 & vacuum \\
\hline LEU09-OP2-Z134 & 17.0 & 4.7 & 950 & 1 & flowing $\mathrm{He}$ & 20 & 1800 & 1 & vacuum \\
\hline LEU09-OP2-Z135 & 17.1 & 4.7 & 950 & 1 & flowing $\mathrm{He}$ & 20 & 1800 & 1 & vacuum \\
\hline LEU09-OP2-Z136 & 16.9 & 4.7 & 950 & 1 & flowing $\mathrm{He}$ & 20 & 1800 & 1 & vacuum \\
\hline LEU09-OP2-Z137 & 17.4 & 4.7 & 950 & 1 & flowing $\mathrm{He}$ & 20 & 1800 & 1 & vacuum \\
\hline LEU09-OP2-Z138 & 17.2 & 4.7 & 950 & 1 & flowing $\mathrm{He}$ & 20 & 1800 & 1 & vacuum \\
\hline LEU09-OP2-Z139 & 16.6 & 4.7 & 950 & 1 & flowing $\mathrm{He}$ & 20 & 1800 & 1 & vacuum \\
\hline LEU09-OP2-Z140 & 17.5 & 4.7 & 950 & 1 & flowing $\mathrm{He}$ & 20 & 1800 & 1 & vacuum \\
\hline LEU09-OP2-Z141 & 16.4 & 4.7 & 950 & 1 & flowing He & 20 & 1800 & 1 & vacuum \\
\hline LEU09-OP2-Z142 & 16.6 & 4.7 & 950 & 1 & flowing He & 20 & 1800 & 1 & vacuum \\
\hline LEU09-OP2-Z143 & 16.2 & 4.7 & 950 & 1 & flowing $\mathrm{He}$ & 20 & 1800 & 1 & vacuum \\
\hline LEU09-OP2-Z144 & 16.5 & 4.7 & 950 & 1 & flowing $\mathrm{He}$ & 20 & 1800 & 1 & vacuum \\
\hline LEU09-OP2-Z145 & 16.0 & 4.7 & 950 & 1 & flowing $\mathrm{He}$ & 20 & 1800 & 1 & vacuum \\
\hline LEU09-OP2-Z146 & 17.0 & 4.7 & 950 & 1 & flowing $\mathrm{He}$ & 20 & 1800 & 1 & vacuum \\
\hline LEU09-OP2-Z147 & 16.9 & 4.7 & 950 & 1 & flowing $\mathrm{He}$ & 20 & 1800 & 1 & vacuum \\
\hline LEU09-OP2-Z148 & 16.8 & 4.7 & 950 & 1 & flowing $\mathrm{He}$ & 20 & 1800 & 1 & vacuum \\
\hline LEU09-OP2-Z149 & 17.0 & 4.7 & 950 & 1 & flowing $\mathrm{He}$ & 20 & 1800 & 1 & vacuum \\
\hline LEU09-OP2-Z150 & 16.6 & 4.7 & 950 & 1 & flowing $\mathrm{He}$ & 20 & 1800 & 1 & vacuum \\
\hline
\end{tabular}


Table 3-2 (cont.): Summary of process conditions used in making LEU09-OP2-Z (AGR-2 UCO Variant) compacts

\begin{tabular}{|c|c|c|c|c|c|c|c|c|c|}
\hline \multirow{2}{*}{ Compact ID } & \multicolumn{5}{|c|}{ Carbonization Parameter } & \multicolumn{4}{|c|}{ Heat-treatment Parameters } \\
\hline & $\begin{array}{l}\text { Molding Pressure } \\
(\mathrm{MPa})\end{array}$ & $\begin{array}{l}\text { Heating Rate } \\
\left({ }^{\circ} \mathrm{C} / \mathrm{min} .\right)\end{array}$ & $\begin{array}{l}\text { Max. Temp. } \\
\left({ }^{\circ} \mathrm{C}\right)\end{array}$ & $\begin{array}{l}\text { Hold Time } \\
\text { (hrs.) }\end{array}$ & Atmosphere & $\begin{array}{l}\text { Heating Rate } \\
\left({ }^{\circ} \mathrm{C} / \mathrm{min} .\right)\end{array}$ & $\begin{array}{l}\text { Max. Temp. } \\
\left({ }^{\circ} \mathrm{C}\right)\end{array}$ & $\begin{array}{l}\text { Hold Time } \\
\text { (hrs.) }\end{array}$ & Atmosphere \\
\hline LEU09-OP2-Z151 & 16.3 & 4.7 & 950 & 1 & flowing $\mathrm{He}$ & 20 & 1800 & 1 & vacuum \\
\hline LEU09-OP2-Z152 & 17.1 & 4.7 & 950 & 1 & flowing $\mathrm{He}$ & 20 & 1800 & 1 & vacuum \\
\hline LEU09-OP2-Z153 & 17.4 & 4.7 & 950 & 1 & flowing $\mathrm{He}$ & 20 & 1800 & 1 & vacuum \\
\hline LEU09-OP2-Z154 & 16.9 & 4.7 & 950 & 1 & flowing $\mathrm{He}$ & 20 & 1800 & 1 & vacuum \\
\hline LEU09-OP2-Z155 & 16.8 & 4.7 & 950 & 1 & flowing $\mathrm{He}$ & 20 & 1800 & 1 & vacuum \\
\hline LEU09-OP2-Z156 & 17.3 & 4.7 & 950 & 1 & flowing $\mathrm{He}$ & 20 & 1800 & 1 & vacuum \\
\hline LEU09-OP2-Z157 & 16.6 & 4.7 & 950 & 1 & flowing $\mathrm{He}$ & 20 & 1800 & 1 & vacuum \\
\hline LEU09-OP2-Z158 & 16.6 & 4.7 & 950 & 1 & flowing $\mathrm{He}$ & 20 & 1800 & 1 & vacuum \\
\hline LEU09-OP2-Z159 & 17.1 & 4.7 & 950 & 1 & flowing $\mathrm{He}$ & 20 & 1800 & 1 & vacuum \\
\hline LEU09-OP2-Z160 & 17.0 & 4.7 & 950 & 1 & flowing $\mathrm{He}$ & 20 & 1800 & 1 & vacuum \\
\hline LEU09-OP2-Z161 & 16.1 & 4.7 & 950 & 1 & flowing $\mathrm{He}$ & 20 & 1800 & 1 & vacuum \\
\hline LEU09-OP2-Z162 & 16.0 & 4.7 & 950 & 1 & flowing $\mathrm{He}$ & 20 & 1800 & 1 & vacuum \\
\hline LEU09-OP2-Z163 & 17.3 & 4.7 & 950 & 1 & flowing $\mathrm{He}$ & 20 & 1800 & 1 & vacuum \\
\hline LEU09-OP2-Z164 & 16.7 & 4.7 & 950 & 1 & flowing $\mathrm{He}$ & 20 & 1800 & 1 & vacuum \\
\hline LEU09-OP2-Z165 & 16.9 & 4.7 & 950 & 1 & flowing $\mathrm{He}$ & 20 & 1800 & 1 & vacuum \\
\hline LEU09-OP2-Z166 & 16.3 & 4.7 & 950 & 1 & flowing $\mathrm{He}$ & 20 & 1800 & 1 & vacuum \\
\hline LEU09-OP2-Z167 & 16.4 & 4.7 & 950 & 1 & flowing $\mathrm{He}$ & 20 & 1800 & 1 & vacuum \\
\hline & & & & & & & & & \\
\hline & & & & & & & & & \\
\hline & & & & & & & & & \\
\hline & & & & & & & & & \\
\hline & & & & & & & & & \\
\hline & & & & & & & & & \\
\hline & & & & & & & & & \\
\hline & & & & & & & & & \\
\hline & & & & & & & & & \\
\hline ask Manager & lew & & & & & Date & $-4-10$ & & \\
\hline
\end{tabular}




\section{Impurity analysis of matrix, resin, and graphites}

The AGR-2 Fuel Specification (SPC-923) puts maximum limits on the elemental impurities Al, $\mathrm{Ca}, \mathrm{Ti}, \mathrm{V}, \mathrm{Cr}, \mathrm{Mn}, \mathrm{Fe}, \mathrm{Co}$, and $\mathrm{Ni}$. The natural graphite, synthetic graphite, and thermosetting resin used to make the matrix/overcoat material may contain these impurities. Therefore, the selection of graphites and resin used to make the matrix must have low concentrations of these impurities to ensure that the compacts made from the matrix will be within specification. Subsequently, part of the compacting development effort was selection and qualification of natural graphite, synthetic graphite, and resin. A graphite or resin was considered "qualified" if it could produce a compact that was within specification on impurities. The AGR-1 compacts showed that compacts could be made from these matrix constituents and pass the impurity specification (see AGR-1 Baseline Compact Lot LEU01-46T-Z data compilation ORNL/TM2006/507, for instance). The qualification process involved receiving natural graphite and synthetic graphite and testing them via glow discharge mass spectrometry (GDMS) in order to establish their initial impurity concentrations. The graphites and resin were then combined to produce matrix that was carbonized and heat treated in powder form. The impurity levels in the heat treated matrix was then also measured by GDMS.

Table 4-1 shows the initial impurity levels for the natural graphite and synthetic graphite that were used to make LEU09-OP2-Z compacts. Natural graphite (Asbury Graphite Mills RD13371), synthetic graphite (SGL Carbon KRB2000), and thermosetting resin (Hexion Durite SC1008-lot LK9DA0008) were combined in a weight ratio of 64:16:20 to make the matrix. Three batches of matrix were produced: RDKrS-050809, RDKrS-052109, and RDKrS-060109. A sample of the RDKrS-050809 matrix was carbonized and heat treated in powder form prior to being tested for impurities by GDMS. The other matrix batches were not tested because they were made up of the same starting materials. Notice that the heat treatment processes significantly reduced impurity levels in the matrix for several elements.

Table 4-1: Matrix constituents that were used in AGR-2 LEU09-OP2-Z compacts

\begin{tabular}{|c|c|c|c|}
\hline \multirow[b]{2}{*}{ Element } & \multicolumn{3}{|c|}{ Impurity concentration (ppm) } \\
\hline & $\begin{array}{l}\text { Natural Graphite- } \\
\text { RD13371 }\end{array}$ & $\begin{array}{c}\text { Synthetic Graphite- } \\
\text { KRB2000 }\end{array}$ & $\begin{array}{l}\text { Heat treated Matrix- } \\
\text { RDKrS-050809 }\end{array}$ \\
\hline Element & Concentration (ppm) & Concentration (ppm) & Concentration (ppm) \\
\hline $\mathrm{Al}$ & 36 & 0.35 & 1.2 \\
\hline $\mathrm{Ca}$ & 9.4 & 0.7 & 0.51 \\
\hline $\mathrm{Ti}$ & 0.43 & 0.06 & 0.92 \\
\hline $\mathrm{V}$ & 0.6 & 0.02 & 8.8 \\
\hline $\mathrm{Cr}$ & 4.5 & $<0.5$ & $<0.5$ \\
\hline $\mathrm{Mn}$ & 0.54 & $<0.05$ & $<0.05$ \\
\hline $\mathrm{Fe}$ & 34 & 1.4 & 0.11 \\
\hline Co & $<0.05$ & 0.25 & $<0.05$ \\
\hline $\mathrm{Ni}$ & 0.37 & 1.2 & $<0.1$ \\
\hline
\end{tabular}

The following pages show the impurity analysis reports for the natural graphite, synthetic graphite, and matrix sample listed in Table 4-1. Also attached is the certificate of analysis for the resin from Hexion. Note that an expiration date was set for the resin of 6 months from the manufacture date. LEU09-OP2-Z compacting was completed on 6/23/09. 

Customer: UT-Battelle Oak Ridge
P.O. \#
MCH4-0191
Date:
27-Dec-03
Job \#
UM4335

Customer ID: Graphite

Shiva ID:

U031218080

AGM RD13371

Element

Concentration

Element

Concentration

[ ppm wt ]

\begin{tabular}{|c|c|c|c|}
\hline $\mathrm{Li}$ & $<0.01$ & $\mathrm{Pd}$ & $<0.1$ \\
\hline $\mathrm{Be}$ & $<0.01$ & $\mathrm{Ag}$ & $<0.1$ \\
\hline$B$ & 0.17 & $\mathrm{Cd}$ & $<0.1$ \\
\hline C & Matrix & $\ln$ & Binder \\
\hline $\mathrm{N}$ & - & $\mathrm{Sn}$ & $<0.5$ \\
\hline $\mathrm{O}$ & - & $\mathrm{Sb}$ & $<0.5$ \\
\hline $\mathrm{F}$ & $<5$ & $\mathrm{Te}$ & $<0.1$ \\
\hline $\mathrm{Na}$ & 2.9 & 1 & $<0.1$ \\
\hline $\mathrm{Mg}$ & 4.8 & $\mathrm{Cs}$ & $<0.1$ \\
\hline $\mathrm{Al}$ & 36 & $\mathrm{Ba}$ & 13 \\
\hline $\mathrm{Si}$ & 240 & $\mathrm{La}$ & $<0.5$ \\
\hline$P$ & 1.6 & $\mathrm{Ce}$ & 0.08 \\
\hline $\mathrm{S}$ & 85 & $\mathrm{Pr}$ & $<0.05$ \\
\hline $\mathrm{Cl}$ & 0.8 & $\mathrm{Nd}$ & $<0.05$ \\
\hline $\mathrm{K}$ & 1.5 & $\mathrm{Sm}$ & $<0.05$ \\
\hline $\mathrm{Ca}$ & 9.4 & Eu & $<0.05$ \\
\hline $\mathrm{Sc}$ & $<0.05$ & $\mathrm{Gd}$ & $<0.05$ \\
\hline $\mathrm{Ti}$ & 0.43 & $\mathrm{~Tb}$ & $<0.05$ \\
\hline $\mathrm{V}$ & 0.6 & Dy & $<0.05$ \\
\hline $\mathrm{Cr}$ & 4.5 & $\mathrm{Ho}$ & $<0.05$ \\
\hline $\mathrm{Mn}$ & 0.54 & $\mathrm{Er}$ & $<0.05$ \\
\hline $\mathrm{Fe}$ & 34 & $\mathrm{Tm}$ & $<0.05$ \\
\hline Co & $<0.05$ & $\mathrm{Yb}$ & $<0.05$ \\
\hline $\mathrm{Ni}$ & 0.37 & $\mathrm{Lu}$ & $<0.05$ \\
\hline $\mathrm{Cu}$ & 1.7 & $\mathrm{Hf}$ & $<0.05$ \\
\hline $\mathrm{Zn}$ & $<0.1$ & $\mathrm{Ta}$ & $<5$ \\
\hline $\mathrm{Ga}$ & $<0.1$ & W & 3.1 \\
\hline $\mathrm{Ge}$ & $<0.1$ & $\mathrm{Re}$ & $<0.05$ \\
\hline As & $<0.1$ & Os & $<0.05$ \\
\hline $\mathrm{Se}$ & $<0.1$ & Ir & $<0.05$ \\
\hline $\mathrm{Br}$ & $<0.1$ & $\mathrm{Pt}$ & $<0.05$ \\
\hline $\mathrm{Rb}$ & $<0.05$ & $\mathrm{Au}$ & $<0.1$ \\
\hline $\mathrm{Sr}$ & 0.19 & $\mathrm{Hg}$ & $<0.5$ \\
\hline$Y$ & 0.95 & $\mathrm{TI}$ & $<0.1$ \\
\hline $\mathrm{Zr}$ & 0.26 & $\mathrm{~Pb}$ & $<0.5$ \\
\hline $\mathrm{Nb}$ & $<0.1$ & $\mathrm{Bi}$ & $<0.1$ \\
\hline Mo & $<0.05$ & Th & $<0.05$ \\
\hline $\mathrm{Ru}$ & $<0.1$ & $U$ & $<0.05$ \\
\hline $\mathrm{Rh}$ & $<0.1$ & & \\
\hline
\end{tabular}


$\begin{array}{ll}\text { Customer: } & \text { UT-Battelle Oak Ridge } \\ \text { Date: } & \text { 26-Dec-03 }\end{array}$

Customer ID: Graphite

SGL
P.O. \#
Job \#

Shiva ID:

Element

Concentration

[ ppm wt ]

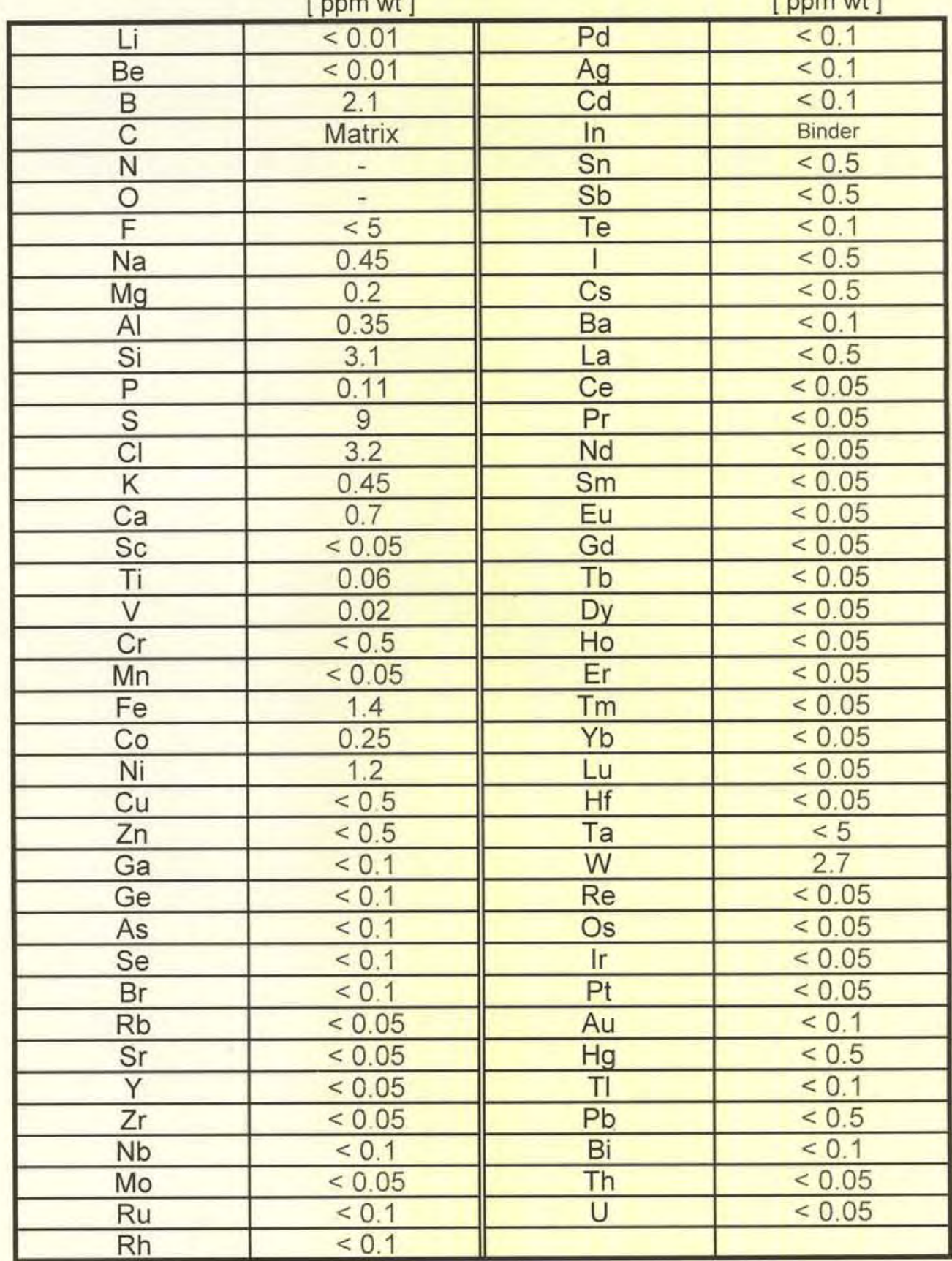

Element

Concentration

[ppm wt]
MCH4-0191

UM4335

U031218078 


$\begin{array}{llll}\text { Customer: } & \text { UT-Battelle Oak Ridge } & \text { P.O.\# } & \text { CC } \\ \text { Date: } & \text { 20-May-09 } & \text { Job\# } & \text { S09×3653 } \\ \text { Customer ID: } & \text { Graphite } & \text { Shiva ID: } & \text { S090514036 } \\ & \text { RDKrS050809 } & & \end{array}$

\begin{tabular}{|c|c|c|c|}
\hline Element & $\begin{array}{c}\text { Concentration } \\
{[\mathrm{ppm} w \mathrm{wt}]}\end{array}$ & Element & $\begin{array}{c}\text { Concentration } \\
{[\mathrm{ppm} \text { wt }]}\end{array}$ \\
\hline $\mathrm{Li}$ & $<0.01$ & $\mathrm{Pd}$ & $<0.1$ \\
\hline $\mathrm{Be}$ & $<0.01$ & $\mathrm{Ag}$ & $<0.1$ \\
\hline$B$ & 0.46 & $\mathrm{Cd}$ & $<0.1$ \\
\hline C & Matrix & $\ln$ & Binder \\
\hline $\mathrm{N}$ & - & Sn & $<0.5$ \\
\hline 0 & - & $\mathrm{Sb}$ & $<0.5$ \\
\hline$F$ & $<5$ & $\mathrm{Te}$ & $<0.1$ \\
\hline $\mathrm{Na}$ & 0.08 & 1 & $<20$ \\
\hline $\mathrm{Mg}$ & $<0.5$ & Cs & $<0.1$ \\
\hline $\mathrm{Al}$ & 1.2 & $\mathrm{Ba}$ & 8.3 \\
\hline $\mathrm{Si}$ & 29 & $\mathrm{La}$ & $<0.5$ \\
\hline$P$ & 0.25 & $\mathrm{Ce}$ & $<0.5$ \\
\hline $\mathrm{S}$ & 2.9 & $\mathrm{Pr}$ & $<0.05$ \\
\hline $\mathrm{Cl}$ & 2.3 & $\mathrm{Nd}$ & $<0.05$ \\
\hline $\mathrm{K}$ & $<0.1$ & $\mathrm{Sm}$ & $<0.05$ \\
\hline $\mathrm{Ca}$ & 0.51 & $\mathrm{Eu}$ & $<0.05$ \\
\hline Sc & $<0.05$ & $\mathrm{Gd}$ & $<0.05$ \\
\hline $\mathrm{Ti}$ & 0.92 & $\mathrm{~Tb}$ & $<0.05$ \\
\hline V & 8.8 & Dy & $<0.05$ \\
\hline $\mathrm{Cr}$ & $<0.5$ & $\mathrm{Ho}$ & $<0.05$ \\
\hline $\mathrm{Mn}$ & $<0.05$ & $\mathrm{Er}$ & $<0.05$ \\
\hline $\mathrm{Fe}$ & 0.11 & $\mathrm{Tm}$ & $<0.05$ \\
\hline Co & $<0.05$ & $\mathrm{Yb}$ & $<0.05$ \\
\hline $\mathrm{Ni}$ & $<0.1$ & $\mathrm{Lu}$ & $<0.05$ \\
\hline $\mathrm{Cu}$ & $<0.1$ & $\mathrm{Hf}$ & $<0.05$ \\
\hline $\mathrm{Zn}$ & $<0.1$ & $\mathrm{Ta}$ & $<5$ \\
\hline $\mathrm{Ga}$ & $<0.1$ & W & 0.41 \\
\hline $\mathrm{Ge}$ & $<0.1$ & $\mathrm{Re}$ & $<0.05$ \\
\hline As & $<0.1$ & Os & $<0.05$ \\
\hline $\mathrm{Se}$ & $<0.1$ & $\mathrm{Ir}$ & $<0.05$ \\
\hline $\mathrm{Br}$ & $<0.1$ & $\mathrm{Pt}$ & $<0.05$ \\
\hline $\mathrm{Rb}$ & $<0.05$ & $\mathrm{Au}$ & $<0.1$ \\
\hline $\mathrm{Sr}$ & $<0.05$ & $\mathrm{Hg}$ & $<0.5$ \\
\hline $\mathrm{Y}$ & $<0.05$ & $\mathrm{TI}$ & $<0.1$ \\
\hline $\mathrm{Zr}$ & 0.65 & $\mathrm{~Pb}$ & $<0.5$ \\
\hline $\mathrm{Nb}$ & $<0.1$ & $\mathrm{Bi}$ & $<0.1$ \\
\hline Mo & $<0.05$ & Th & $<0.05$ \\
\hline $\mathrm{Ru}$ & $<0.1$ & $U$ & $<0.05$ \\
\hline $\mathrm{Rh}$ & $<0.1$ & & \\
\hline
\end{tabular}




\section{HEXION Hexion Specialty Ch

Customer \#: 32710

Customer Address:

CAPITAL RESIN CORPORATION

324 DERING AVENUE

COLUMBUS OH 43207

USA

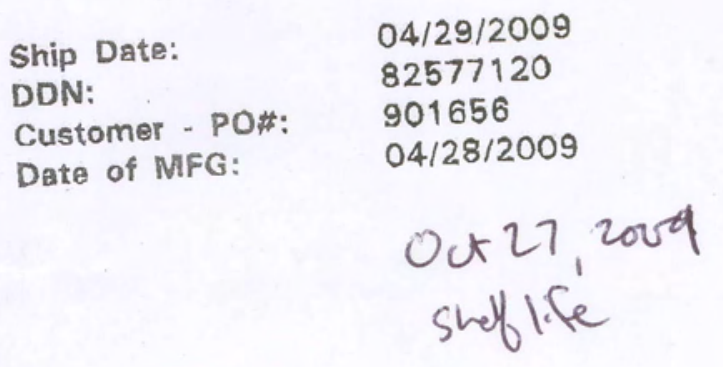

$\begin{array}{ll}\text { Attention: } & \text { KAY FREY } \\ \text { Customer Phone \#: } & 614-445-7177 \\ \text { Customer Fax \#: } & 614-445-7290 \\ & \\ \text { SAP Product \#: } & 305922\end{array}$

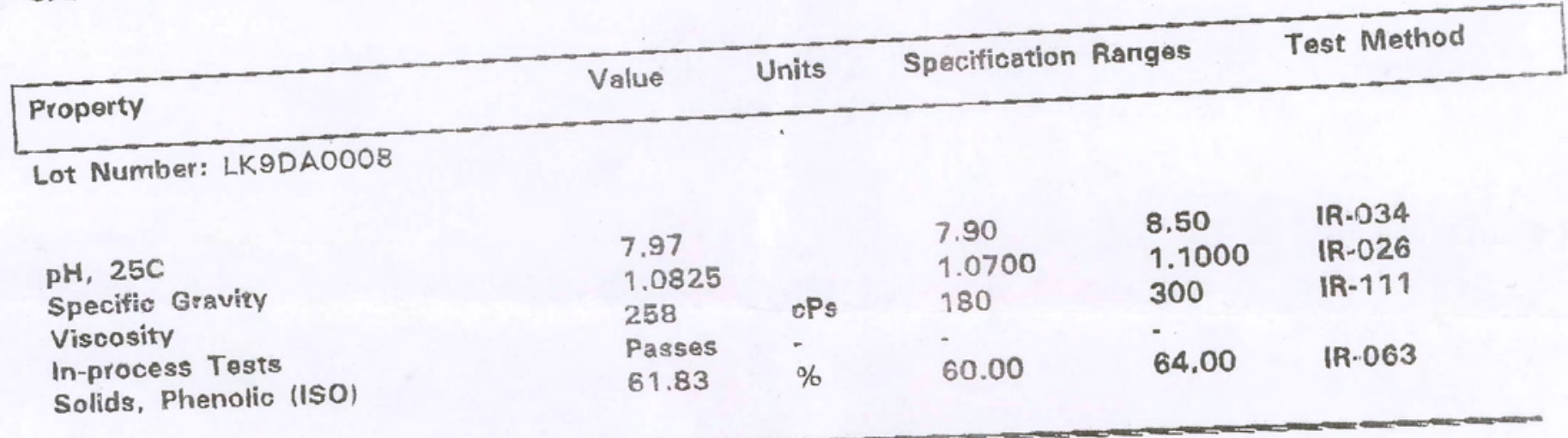

Product Description: Durite SC-1008

DS3271/450\# 


\section{Characterization of coated particles}

This section contains characterization data on the TRISO particle lot LEU09. The data was obtained according to product inspection plan AGR-CHAR-PIP-10R2, "Product Inspection Plan for AGR-2 Particles for Compacting - Preliminary Measurements." The data obtained by this inspection plan is used in support of compact fabrication and for input into measurements made for compact acceptance testing. There are no direct specifications for the measured parameters.

LEU09 particles were obtained from the G73J-14-93073A coated particle batch by riffling a sample using a chute splitter. Prior to performing AGR-CHAR-PIP-10R2, the particles were sorted by size using a roller-micrometer (see Appendix C) and then washed in methanol. No uncoated kernels or coating fragments were found, but 14 small or highly faceted particles were segregated out and removed. PIP-10 calls for measurement of average particle weight, diameter, envelope volume, and uranium content. OPyC open porosity is also obtained as part of the envelope volume analysis and reported for information only. The plan also calls for riffling of 20 gram aliquots for use as overcoater charges. Riffling at ORNL was done using a 10 position rotary riffler. After riffling out the characterization samples, forty-eight overcoater charges were prepared and labeled LEU09-Y01 through LEU09-Y48. Additional ORNL characterization performed on another sample taken from G73J-14-93073A is provided in ORNL/TM-2008/134, "Data Compilation for AGR-2 UCO Variant Coated Particle Batch G73J-14-93073A."

The following pages show the inspection report form (IRF-10) for the LEU09 particles. Following the IRF-10 inspection report form, which summarizes the results, are the individual data report forms for the measurements that were performed. 


\section{Inspection Report Form IRF-10: AGR-2 Particles for Compacting - Preliminary Measurements}

Procedure: AGR-CHAR-PIP-10 Rev. 2

Coated particle composite ID: LEU09

Coated particle composite description: AGR-2 UCO Variant Fuel, from G73J-14-93073A

\begin{tabular}{|c|c|c|c|c|c|c|c|c|c|}
\hline \multirow[b]{2}{*}{ Property } & \multicolumn{4}{|c|}{ Measured Data } & \multirow{2}{*}{$\begin{array}{l}\text { Specification } \\
\text { INL SPC-923 }\end{array}$} & \multirow[b]{2}{*}{ Acceptance Criteria } & \multirow{2}{*}{$\begin{array}{l}\text { Acceptance } \\
\text { Test Value }\end{array}$} & \multirow{2}{*}{$\begin{array}{c}\text { Pass } \\
\text { or } \\
\text { fail } \\
\end{array}$} & \multirow{2}{*}{$\begin{array}{c}\text { Data } \\
\text { Records }\end{array}$} \\
\hline & $\begin{array}{c}\text { Mean } \\
(x)\end{array}$ & $\begin{array}{l}\text { Std. Dev. } \\
\text { (s) }\end{array}$ & $\begin{array}{c}\# \text { measured } \\
(n)\end{array}$ & $t$ value & & & & & \\
\hline Particle diameter $(\mu \mathrm{m})$ & 873.2 & 23 & 2003 & 1.646 & \multicolumn{4}{|c|}{ Not Applicable } & $\begin{array}{l}\text { DRF-07 } \\
\text { DRF-10 }\end{array}$ \\
\hline Average particle weight $(g)$ & $1.032 \mathrm{E}-03$ & & & & \multicolumn{4}{|c|}{ Not Applicable } & DRF-22 \\
\hline Average particle envelope volume $\left(\mathrm{cm}^{3}\right)$ & $3.43 \mathrm{E}-04$ & & & & \multicolumn{4}{|c|}{ Not Applicable } & DRF-31 \\
\hline OPyC open porosity $\left(\mathrm{ml} / \mathrm{m}^{2}\right)$ & 0.234 & & & & \multicolumn{4}{|c|}{ Not Applicable } & DRF-31 \\
\hline Average uranium per particle $(g)$ & $3.964 E-04$ & & & & \multicolumn{4}{|c|}{ Not Applicable } & DRF-35 \\
\hline
\end{tabular}

Comments
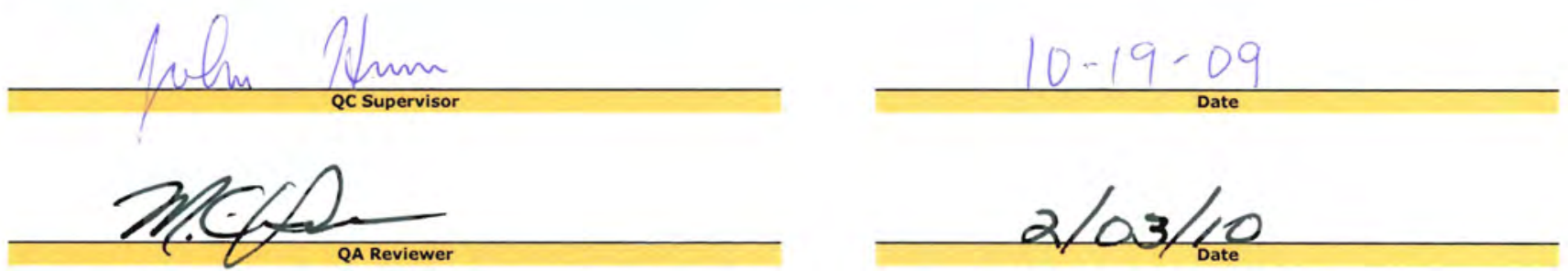
Data Report Form DRF-07: Imaging of Particle Diameter and Aspect Ratio Using an Optical Microscope System Procedure: AGR-CHAR-DAM-07 Rev. 1

Operator: Andrew Kercher

Sample ID: LEU09-B01

Sample Description: AGR-2 UCO Variant, from G73J-14-93073A

Folder name containing images: \\Mc-agr\AGR\ImageProcessing\P09051401\

DMR Calibration Expiration Date: 10/28/2009

Stage Micrometer Calibration Expiration Date:2/10/2014

Measured Value for $1200 \mu \mathrm{m}$ in Stage Micrometer Image: $1200 . \mu \mathrm{m}$
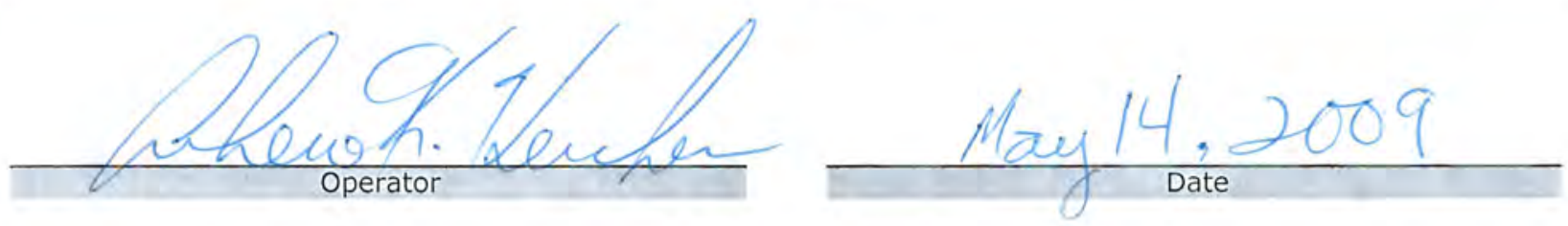
Data Report Form DRF-10A: Measurement of Particle Diameter

\begin{tabular}{|c|c|}
\hline \multicolumn{2}{|l|}{ Procedure: AGR-CHAR-DAM-10 Rev. 2} \\
\hline Operator: Andrew K. Kercher & \\
\hline 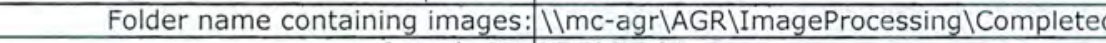 & $09051401 \backslash$ \\
\hline Sample ID:|LEU09-B01 & \\
\hline Sample Description: AGR-2 UCO Variant, from G73J-14-93073A & \\
\hline 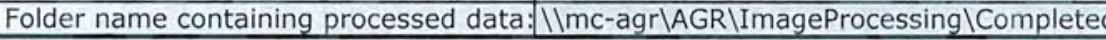 & 09051401 outpu \\
\hline Number of particles analyzed: & 2003 \\
\hline Mean of the average diameter of each particle $(\mu \mathrm{m})$ & 873.2 \\
\hline Standard deviation in the average diameter of each particle $(\mu \mathrm{m})$ & 23 \\
\hline
\end{tabular}

Distribution of the average particle diameter (top binned)

\begin{tabular}{|c|c|}
\hline Mean Diameter $(\mu \mathrm{m})$ & Frequency \\
\hline \hline$<800$ & 0 \\
\hline 810 & 2 \\
\hline 820 & 7 \\
\hline 830 & 35 \\
\hline 840 & 122 \\
\hline 850 & 160 \\
\hline 860 & 279 \\
\hline 870 & 295 \\
\hline 880 & 338 \\
\hline 890 & 285 \\
\hline 900 & 227 \\
\hline 910 & 135 \\
\hline 920 & 66 \\
\hline 930 & 28 \\
\hline 940 & 15 \\
\hline 950 & 8 \\
\hline$>950$ & 1 \\
\hline
\end{tabular}
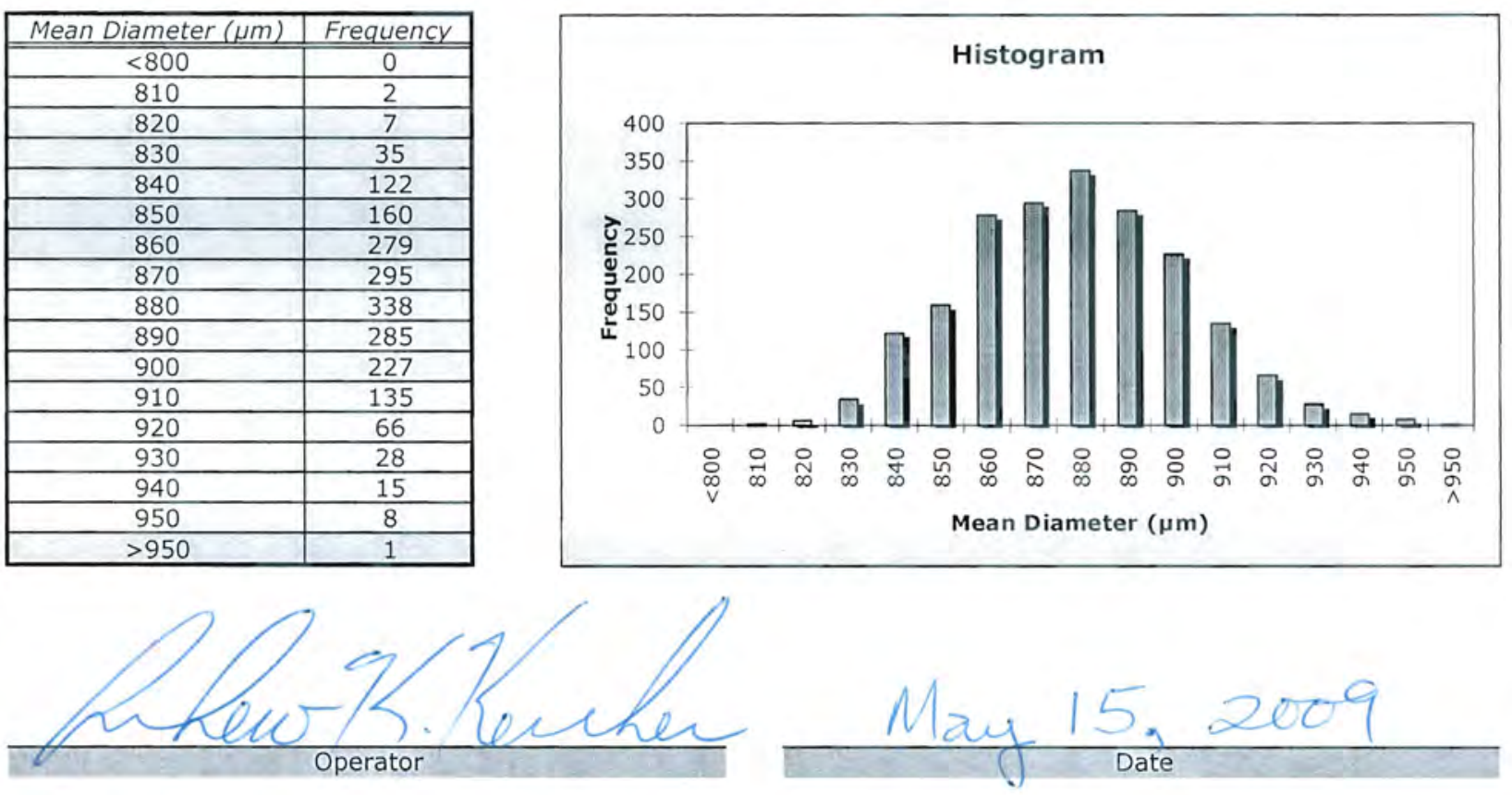
Data Report Form DRF-10B: Measurement of Particle Aspect Ratio (Dmax/Dmin)

\begin{tabular}{|c|c|}
\hline Procedure: & AGR-CHAR-DAM-10 Rev. 2 \\
\hline Operator: & Andrew K. Kercher \\
\hline Folder name containing images: & 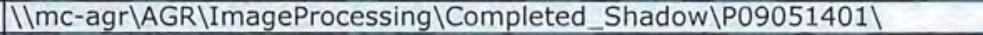 \\
\hline Sample ID: & LEU09-B01 \\
\hline Sample Description: & AGR-2 UCO Variant, from G73J-14-93073A \\
\hline Folder name containing processed data: & 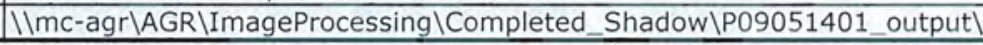 \\
\hline
\end{tabular}

\begin{tabular}{|r|c|}
\hline Number of particles analyzed: & 2003 \\
\hline Number of particles with aspect ratio $\geq 1.14$ & 0 \\
\hline Average particle aspect ratio: & 1.052 \\
\hline
\end{tabular}

Distribution of the aspect ratio (top binned)

\begin{tabular}{|c|c|}
\hline Aspect Ratio (D) & Frequency \\
\hline \hline 1.005 & 0 \\
\hline 1.010 & 0 \\
\hline 1.015 & 3 \\
\hline 1.020 & 15 \\
\hline 1.025 & 55 \\
\hline 1.030 & 89 \\
\hline 1.035 & 150 \\
\hline 1.040 & 195 \\
\hline 1.045 & 219 \\
\hline 1.050 & 240 \\
\hline 1.055 & 244 \\
\hline 1.060 & 232 \\
\hline 1.065 & 148 \\
\hline 1.070 & 126 \\
\hline 1.075 & 117 \\
\hline 1.080 & 57 \\
\hline 1.085 & 44 \\
\hline 1.090 & 30 \\
\hline 1.095 & 13 \\
\hline 1.100 & 13 \\
\hline 1.105 & 4 \\
\hline 1.110 & 4 \\
\hline 1.115 & 1 \\
\hline 1.120 & 3 \\
\hline 1.125 & 1 \\
\hline 1.130 & 0 \\
\hline 1.135 & 0 \\
\hline 1.140 & 0 \\
\hline$>1.14$ & 0 \\
\hline
\end{tabular}
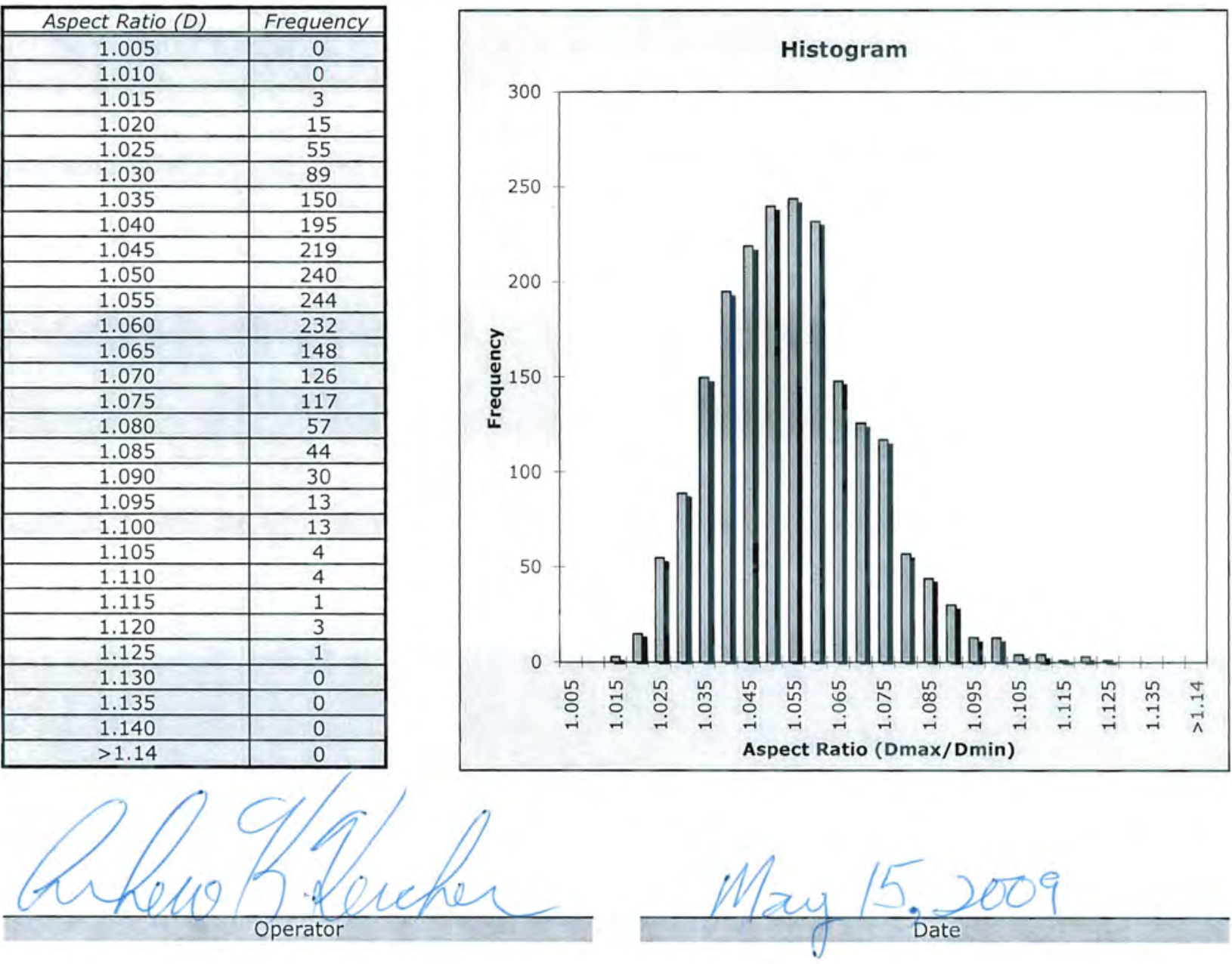
ORNL/TM-2010/017

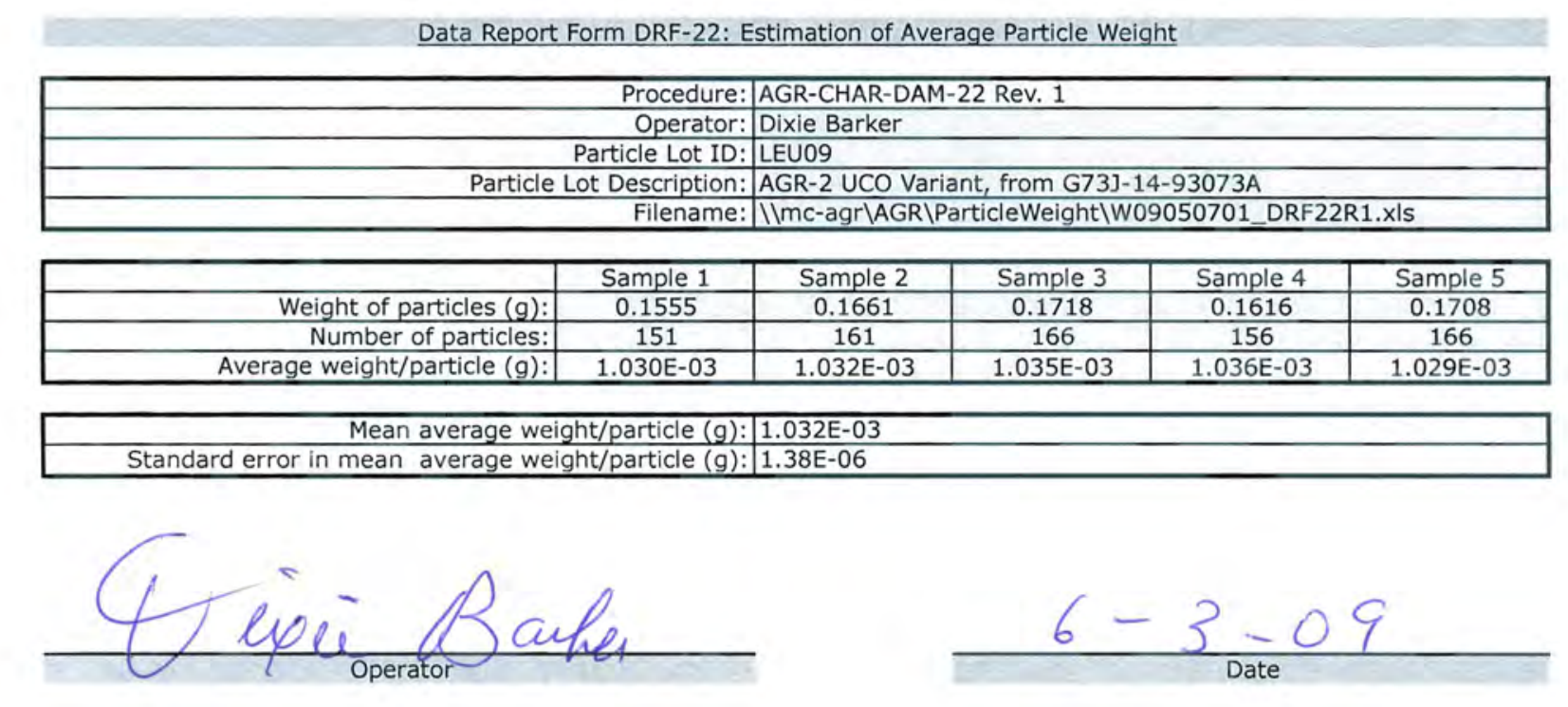




\section{Data Report Form DRF-31: Measurement of Open Porosity using a Mercury Porosimeter}

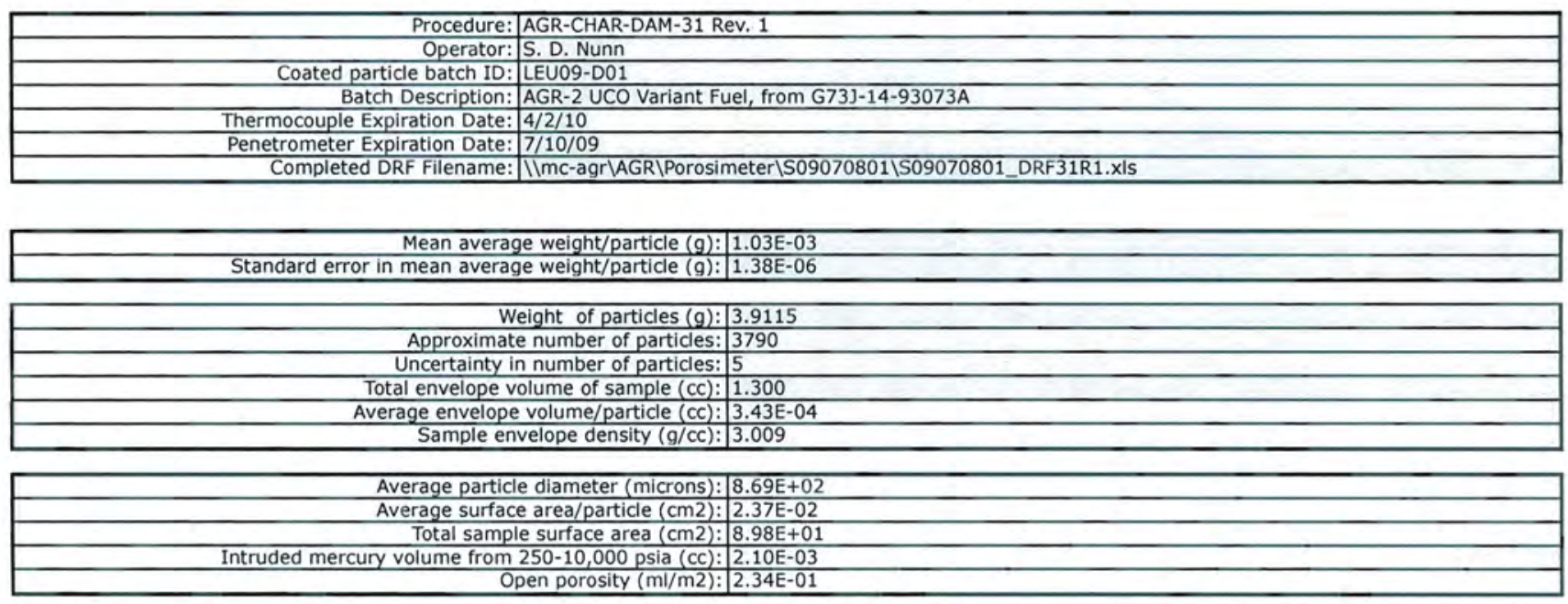


Data Report Form DRF-35: Fuel Particle Uranium Loading

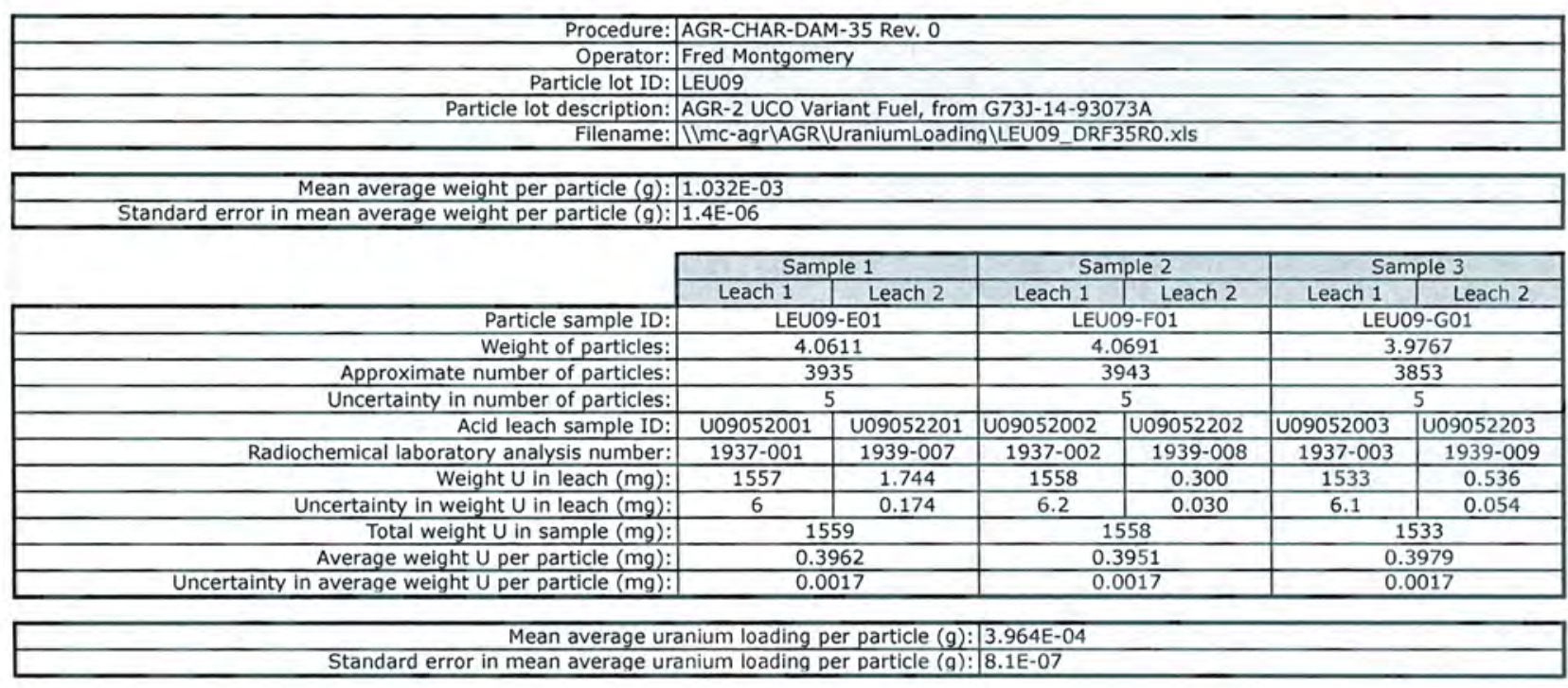

\section{Comments}

Leach 1 was analyzed by Davies-Gray titration method. Leach 2 was analyzed by ICP-MS, due to low U concentration.

Davies-Gray: initial known $U$ recovery $=100.66 \%$; final known $U$ recovery $=100.87 \%$; blind titration $U$ recovery $=100.33 \%$

Uncertainty in Davies-Gray $(0.4 \%)$ based on average of measured \% recovery data for LEU06,07,08,09.

Data checked by FCM against official results of analyses for RMAL1937 on 6/19/2009 and against RMAL1939 on 7/20/2009

\section{Fred C. Montgomery}

$10-19-2009$ 


\section{Characterization of overcoated particles}

This section contains characterization data on the overcoated particle lot LEU09-OP2. The data was obtained according to product inspection plan AGR-CHAR-PIP-11R0, "Product Inspection Plan for AGR-2 Overcoated Particles for Compacting." The data obtained by this inspection plan is used in support of compact fabrication and for input into measurements made for compact acceptance testing. There are no direct specifications for the measured parameters.

After overcoating, the overcoated particles from LEU09-Y01 through LEU09-Y41 were combined and homogenized into an overcoated particle composite. The overcoated particle composite was labeled LEU09-OP2 and AGR-CHAR-PIP-11R0 was completed. This plan calls for measurement of average overcoated particle weight and diameter. The plan also calls for riffling of compact charges for pressing. One hundred and eighty five compact charges were prepared and labeled LEU09-OP2-G001 through LEU09-OP2-G185. A record of the weight of each compact charge can be found on data report form DRF-24D, in section 7.

The following pages show the inspection report form (IRF-11) for the LEU09-OP2 overcoated particles. Following the IRF-11 inspection report form, which summarizes the results, are the individual data report forms for the measurements that were performed.

The average thickness of the overcoat can be estimated from the increase in the average particle size after overcoating, $(1303 \mu \mathrm{m}-873 \mu \mathrm{m}) / 2=215 \mu \mathrm{m}$. The increase in average particle weight was $(2.010 \mathrm{mg}-1.032 \mathrm{mg})=0.978 \mathrm{mg}$. From these values, the average density of the overcoating prior to compacting can be estimated to be $1.21 \mathrm{~g} / \mathrm{cm}^{3}$. 
ORNL/TM-2010/017

Inspection Report Form IRF-11: AGR-2 Overcoated Particles for Compacting

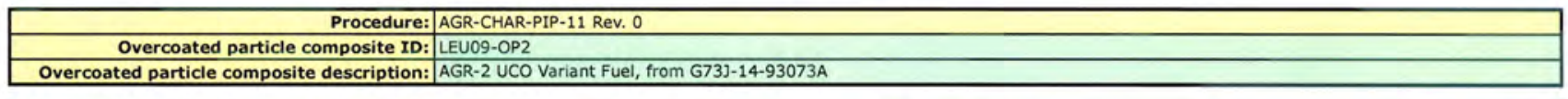

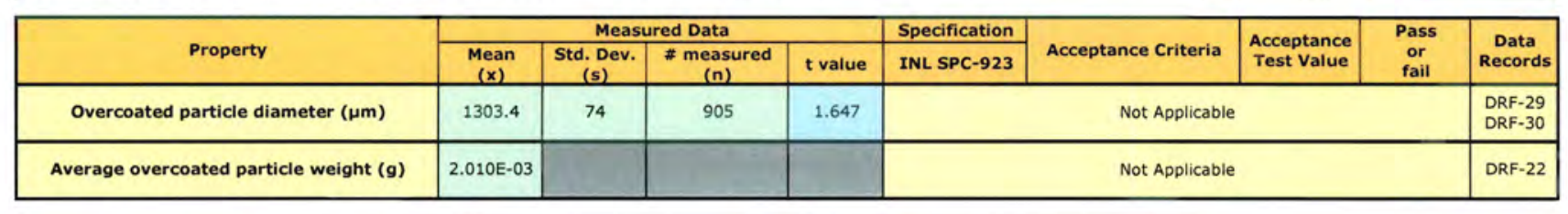
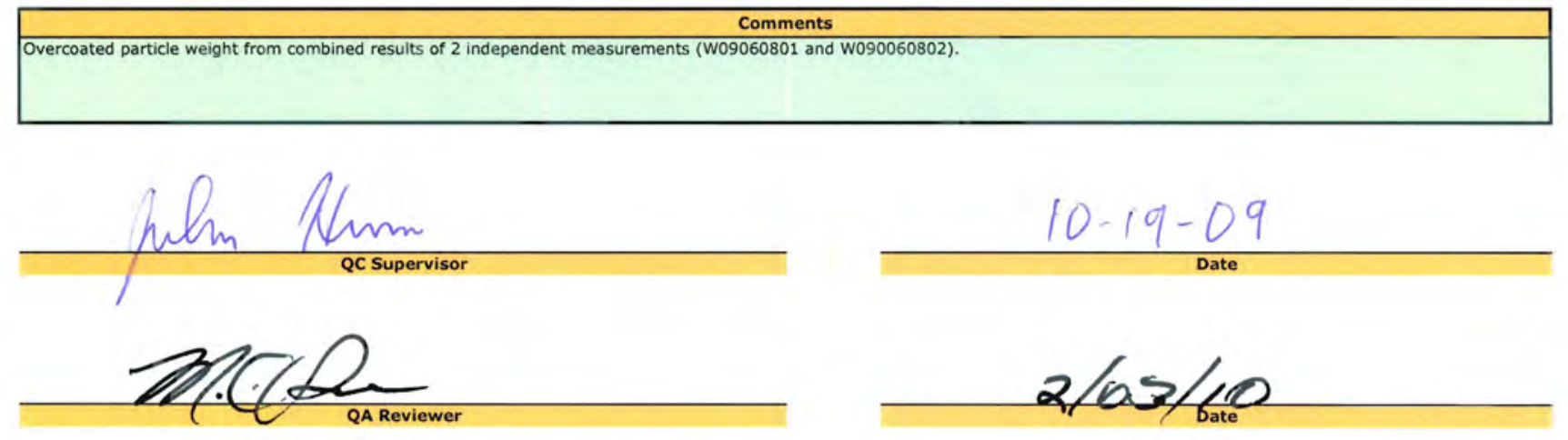

34 


\section{Data Report Form DRF-29: Imaging of Overcoated Particle Diameter and Aspect Ratio Using an Optical Microscope System}

\begin{tabular}{|c|c|}
\hline Procedure: & AGR-CHAR-DAM-29 Rev. 1 \\
\hline Operator: & Andrew K. Kercher \\
\hline Sample ID: & LEU09-OP2-B01 \\
\hline Sample Description: & AGR-2 UCO Variant, from G73J-14-93073A \\
\hline Folder name containing images: & 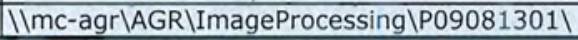 \\
\hline
\end{tabular}

\begin{tabular}{|r|l|}
\hline DMR Calibration Expiration Date: & $10 / 28 / 2009$ \\
\hline Stage Micrometer Calibration Expiration Date: & $2 / 10 / 2014$ \\
\hline Measured Value for $2500 \mu \mathrm{m}$ in Stage Micrometer Image: & $2500 . \mu \mathrm{m}$ \\
\hline
\end{tabular}

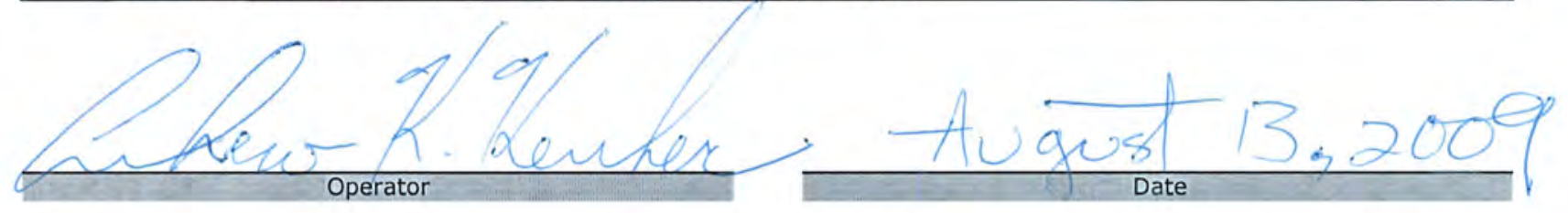




\section{Data Report Form DRF-30A: Measurement of Over-coated Particle Diameter}

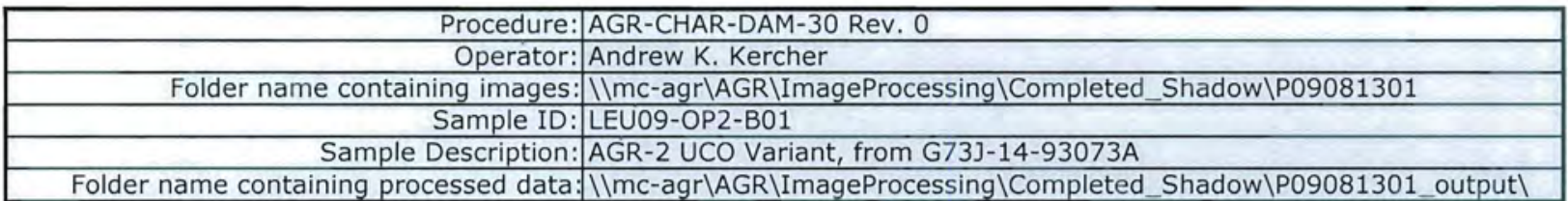

\begin{tabular}{|r|c|}
\hline Number of particles analyzed: & 905 \\
\hline Mean of the average diameter of each particle $(\mu \mathrm{m})$ & 1303.4 \\
\hline Standard deviation in the average diameter of each particle $(\mu \mathrm{m})$ & 74 \\
\hline
\end{tabular}

\section{Distribution of the average particle diameter (top binned)}

\begin{tabular}{|c|c|}
\hline Mean Diameter $(\mu \mathrm{m})$ & Frequency \\
\hline \hline$<1040$ & 3 \\
\hline 1080 & 0 \\
\hline 1120 & 2 \\
\hline 1160 & 8 \\
\hline 1200 & 37 \\
\hline 1240 & 106 \\
\hline 1280 & 193 \\
\hline 1320 & 197 \\
\hline 1360 & 195 \\
\hline 1400 & 91 \\
\hline 1440 & 34 \\
\hline 1480 & 22 \\
\hline 1520 & 10 \\
\hline 1560 & 5 \\
\hline 1600 & 1 \\
\hline 1640 & 1 \\
\hline$>1640$ & 0 \\
\hline & \\
\hline &
\end{tabular}

Histogram

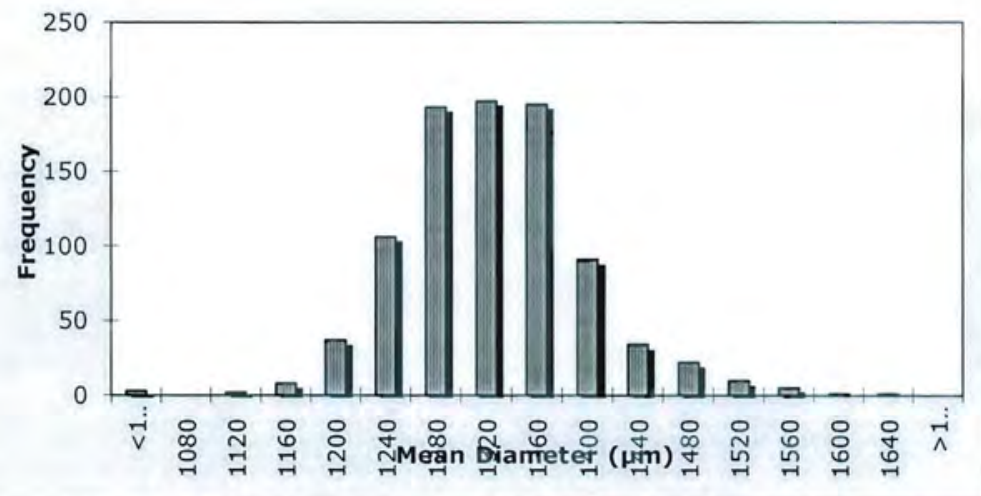

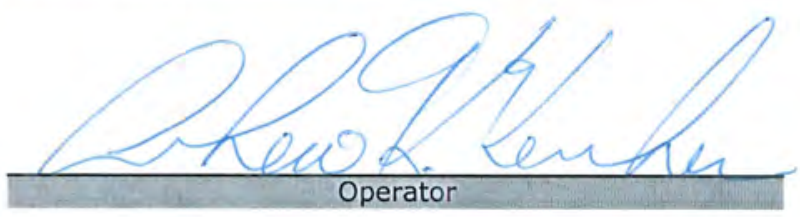

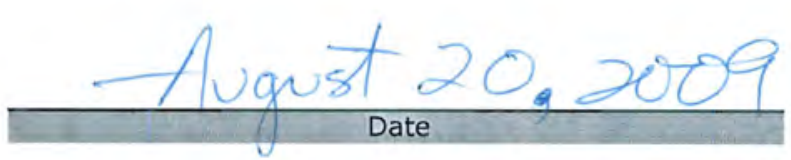


Data Report Form DRF-30B: Measurement of Over-coated Particle Aspect Ratio (Dmax/Dmin)

\begin{tabular}{|c|c|}
\hline Procedure: & AGR-CHAR-DAM-30 Rev. 0 \\
\hline Operator: & Andrew K. Kercher \\
\hline Folder name containing images: & 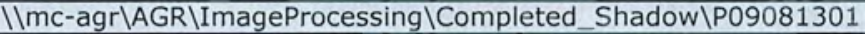 \\
\hline Sample ID: & LEU09-OP2-B01 \\
\hline Sample Description: & AGR-2 UCO Variant, from G73J-14-93073A \\
\hline
\end{tabular}

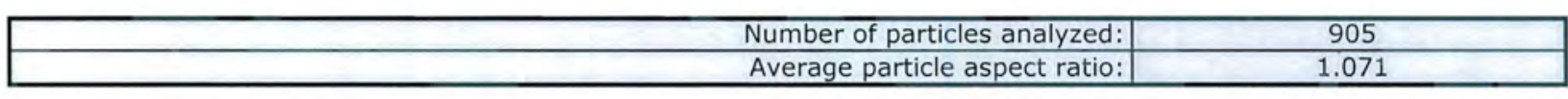

Distribution of the aspect ratio (top binned)

\begin{tabular}{|c|c|}
\hline Aspect Ratio (D) & Frequency \\
\hline 1.005 & 0 \\
\hline 1.010 & 0 \\
\hline 1.015 & 1 \\
\hline 1.020 & 1 \\
\hline 1.025 & 6 \\
\hline 1.030 & 15 \\
\hline 1.035 & 27 \\
\hline 1.040 & 40 \\
\hline 1.045 & 57 \\
\hline 1.050 & 62 \\
\hline 1.055 & 82 \\
\hline 1.060 & 83 \\
\hline 1.065 & 77 \\
\hline 1.070 & 78 \\
\hline 1.075 & 65 \\
\hline 1.080 & 48 \\
\hline 1.085 & 48 \\
\hline 1.090 & 41 \\
\hline 1.095 & 30 \\
\hline 1.100 & 34 \\
\hline 1.105 & 18 \\
\hline 1.110 & 14 \\
\hline 1.115 & 13 \\
\hline 1.120 & 15 \\
\hline 1.125 & 6 \\
\hline 1.130 & 13 \\
\hline 1.135 & 6 \\
\hline 1.140 & 4 \\
\hline$>1.14$ & 21 \\
\hline
\end{tabular}
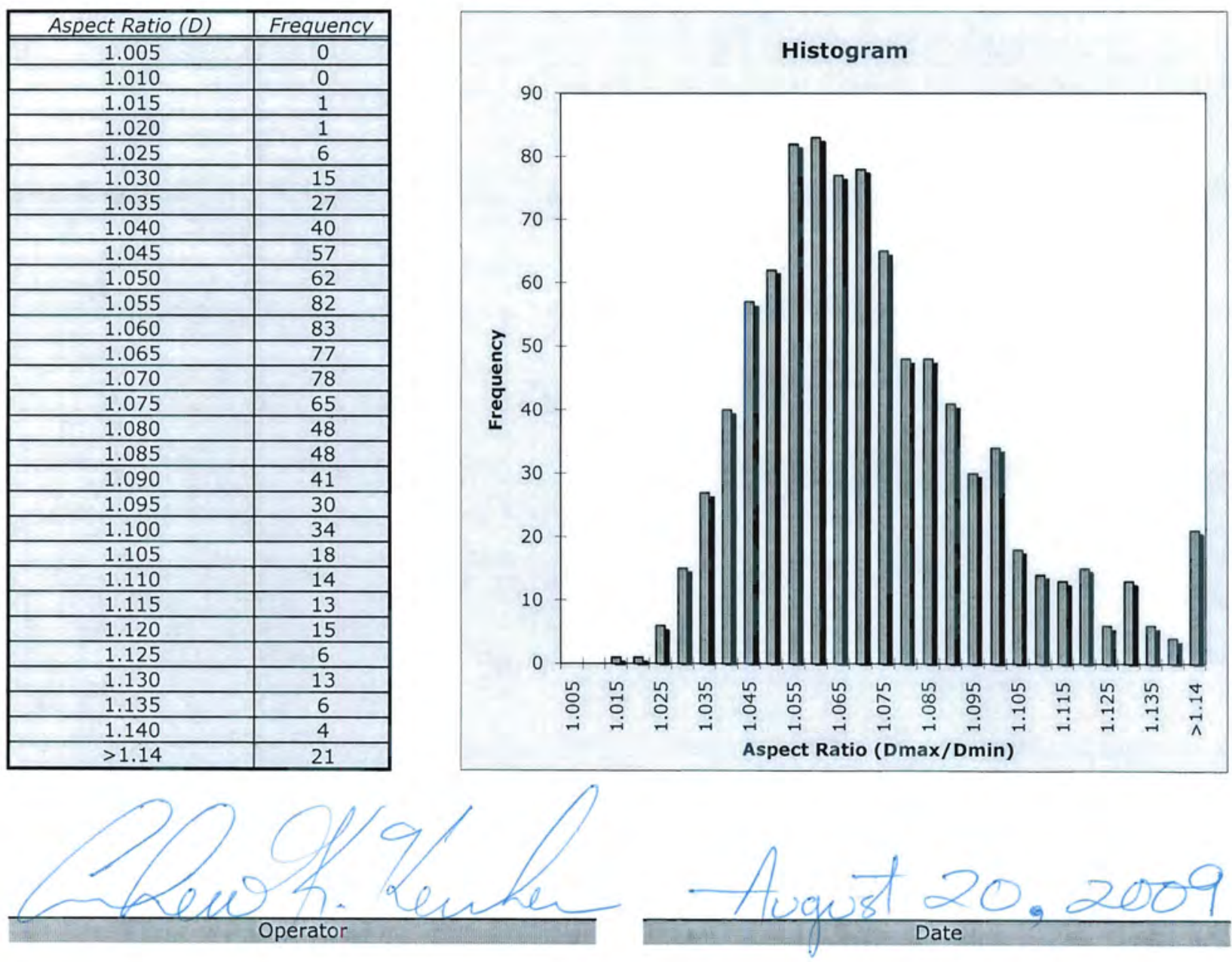
Data Report Form DRF-22: Estimation of Average Particle Weight

\begin{tabular}{|r|l|}
\hline Procedure: & AGR-CHAR-DAM-22 Rev. 1 \\
\hline Operator: & Dixie Barker \\
\hline Particle Lot ID: & LEU09-OP2 \\
\hline Particle Lot Description: & AGR-2 UCO Variant, from G73J-14-93073A \\
\hline Filename: & |ImC-agr\AGR\ParticleWeight|W09060801_DRF22R1.xIs \\
\hline
\end{tabular}

\begin{tabular}{|r|c|c|c|c|c|}
\hline & Sample 1 & Sample 2 & Sample 3 & Sample 4 & Sample 5 \\
\hline Weight of particles (g): & 0.3009 & 0.3340 & 0.2682 & 0.2966 & 0.3240 \\
\hline Number of particles: & 151 & 162 & 132 & 149 & 162 \\
\hline Average weight/particle (g): & $1.993 \mathrm{E}-03$ & $2.062 \mathrm{E}-03$ & $2.032 \mathrm{E}-03$ & $1.991 \mathrm{E}-03$ & $2.000 \mathrm{E}-03$ \\
\hline
\end{tabular}

Mean average weight/particle $(\mathrm{g}): 2.015 \mathrm{E}-03$

Standard error in mean average weight/particle $(\mathrm{g}): 1.38 \mathrm{E}-05$

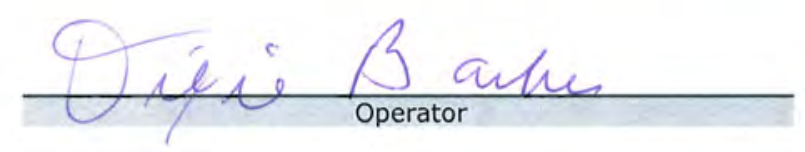

$$
\frac{6-8-09}{\text { Date }}
$$


Data Report Form DRF-22: Estimation of Average Particle Weight

\begin{tabular}{|r|l|}
\hline Procedure: & AGR-CHAR-DAM-22 Rev. 1 \\
\hline Operator: & Dixie Barker \\
\hline Particle Lot ID: & LEU09-OP2 \\
\hline Particle Lot Description: & AGR-2 UCO Variant, from G73J-14-93073A \\
\hline Filename: & \Imc-agr\AGR\ParticleWeight\W09060802_DRF22R1.xIs \\
\hline
\end{tabular}

\begin{tabular}{|r|c|c|c|c|c|}
\hline & Sample 6 & Sample 7 & Sample 8 & Sample 9 & Sample 10 \\
\hline Weight of particles (g): & 0.2609 & 0.2581 & 0.2918 & 0.2500 & 0.3063 \\
\hline Number of particles: & 129 & 131 & 144 & 125 & 153 \\
\hline Average weight/particle (g): & $2.022 \mathrm{E}-03$ & $1.970 \mathrm{E}-03$ & $2.026 \mathrm{E}-03$ & $2.000 \mathrm{E}-03$ & $2.002 \mathrm{E}-03$ \\
\hline
\end{tabular}

Mean average weight/particle $(\mathrm{g}): 2.004 \mathrm{E}-03$

Standard error in mean average weight/particle $(\mathrm{g}): 1.00 \mathrm{E}-05$
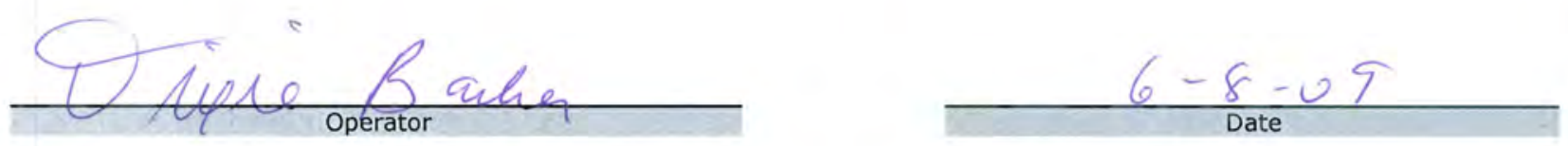


\section{Characterization of compacts}

This section contains acceptance testing data on the compact lot LEU09-OP2-Z. The data was obtained according to product inspection plan AGR-CHAR-PIP-12R1, "Product Inspection Plan for AGR-2 UCO Fuel Compact Lots." This compact lot was determined to not fully satisfy the specifications in section 3.3 of the AGR-2 Fuel Specification (INL SPC-923, Rev. 3). All specification were satisfied except for a higher than allowed fraction of exposed uranium, discussed below. However, it was determined by the program that the observed uranium contamination fraction non-conformance was acceptable for "use as is" in the AGR-2 irradiation test. This disposition was documented on INL NCR-44791.

After compacting, 167 compacts were selected from LEU09-OP2-G001 through G185 for use. Compacts with obvious processing defects, chips, or undesirable dimensions were sorted out and not included in the 167 compacts selected for the final fuel compact lot. This down-select was part of the compacting process and was performed prior to random selection of compacts for acceptance testing. It should be understood that the results in this section and the acceptance testing are only relevant for the final 167 compact lot from which random representative samples were drawn for characterization. As instructed in AGR-CHAR-PIP-12R1, these 167 compacts were randomized and relabeled as LEU09-OP2-Z001 through Z167. A record of the original Gnumber for each Z-numbered compact can be found on data report form DRF-24C, in this section. After relabeling, the compacts were characterized for product acceptance according to product inspection plan PIP-12. This plan calls for measurement of compact length, diameter, mass, matrix density, uranium content, impurity content, and determination of defect fractions for exposed uranium, defective $\mathrm{SiC}$, uranium dispersion due to defective IPyC, and defective $\mathrm{OPyC}$.

The following pages show the inspection report forms (IRF-12A, IRF-12B, IRF-12C, IRF-12D) for the LEU09-OP2-Z compacts. Following the IRF-12 inspection report forms, which summarize the results, are the individual data report forms for the measurements that were performed. Note that the leach-burn-leach (LBL) analysis is performed on sets of 20 compacts at a time, in four sample groups with 5 compacts in each sample. Inspection report forms IRF-12B, IRF-12C, and IRF-12D summarize the results from each set of 20 compacts. Inspection report form IRF-12A summarizes all the analyses. The mean and standard deviation for the impurity analyses (IRF-12B), the uranium contamination fraction or effective number of exposed kernels before the burn (IRF-12C), and the defective SiC coating fraction or number of exposed kernels after the burn (IRF-12D) are calculated from the combined results of all the relevant sample groups. These combined results, which are then entered into IRF-12A, are provided in Table 7-1 and Table 7-2 below. Note that, for some sets of 20 compacts, only pre-burn leach analysis is performed. This is because the uranium contamination defect fraction is specified at a lower allowable maximum population, which requires a greater number of particles be analyzed in order to demonstrate product acceptance. Pre-burn leach analysis was completed on 100 compacts. Post-burn leach analysis was completed on 80 compacts. The third set of 20 compacts did not undergo post-burn leach analysis, and therefore did not contribute to the total impurity analysis or defective $\mathrm{SiC}$ analysis. 
Table 7-1: Summary of impurity analysis for LEU09-OP2-Z compacts

\begin{tabular}{|c|c|c|c|c|c|c|c|c|c|c|}
\hline Compact ID numbers: & $\begin{array}{c}011,152, \\
001,068, \\
046\end{array}$ & $\begin{array}{c}067,081, \\
019,008, \\
032\end{array}$ & $\begin{array}{c}039,036, \\
155,012, \\
117\end{array}$ & $\begin{array}{c}031,149, \\
093,147, \\
072\end{array}$ & $\begin{array}{c}051,086, \\
143,097, \\
033\end{array}$ & $\begin{array}{c}030,024, \\
090,013, \\
010\end{array}$ & $\begin{array}{c}021,035 \\
121,107, \\
061\end{array}$ & $\begin{array}{c}151,003, \\
058,080, \\
009\end{array}$ & Mean & $\begin{array}{c}\text { Standard } \\
\text { Deviation }\end{array}$ \\
\hline Number of compacts: & 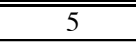 & 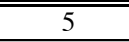 & 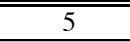 & 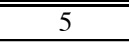 & 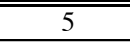 & 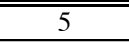 & 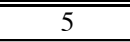 & 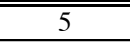 & & \\
\hline \multicolumn{11}{|l|}{ Iron } \\
\hline Deconsolidation-leach (DRF-26A) $(\mu \mathrm{g})$ : & 10.71 & 10.55 & 10.92 & 9.76 & 10.75 & 10.96 & 58.59 & 10.84 & & \\
\hline Burn-leach (DRF-26B) $(\mu \mathrm{g})$ & 6.17 & 24.50 & 5.11 & 5.24 & 5.39 & 7.11 & 5.22 & 5.58 & & \\
\hline Total leached $(\mu \mathrm{g})$ : & 16.88 & 35.04 & 16.03 & 15.01 & 16.15 & 18.07 & 63.81 & 16.42 & & \\
\hline Fe outside $\mathrm{SiC}(\mu \mathrm{g} /$ compact $):$ & 3.38 & 7.01 & 3.21 & 3.00 & $\mathbf{3 . 2 3}$ & 3.61 & 12.76 & 3.28 & \multicolumn{2}{|c|}{ Continued on next page } \\
\hline \multicolumn{11}{|l|}{ Chromium } \\
\hline Deconsolidation-leach (DRF-26A) $(\mu \mathrm{g})$ : & 1.45 & 1.14 & 2.30 & 1.85 & 1.33 & 1.90 & 1.10 & 0.71 & & \\
\hline Burn-leach (DRF-26B) $(\mu \mathrm{g})$ : & 1.26 & 1.24 & 1.16 & 1.15 & 1.25 & 1.29 & 1.18 & 1.28 & & \\
\hline Total leached $(\mu \mathrm{g})$ : & 2.71 & 2.38 & 3.46 & 3.00 & 2.58 & 3.19 & 2.27 & 1.99 & & \\
\hline Cr outside SiC ( $\mu \mathrm{g} /$ compact): & 0.54 & 0.48 & 0.69 & $\mathbf{0 . 6 0}$ & 0.52 & 0.64 & $\mathbf{0 . 4 5}$ & 0.40 & \multicolumn{2}{|c|}{ Continued on next page } \\
\hline \multicolumn{11}{|l|}{ Manganese } \\
\hline Deconsolidation-leach (DRF-26A) $(\mu \mathrm{g})$ : & 0.50 & 0.49 & 0.51 & 0.45 & 0.50 & 0.51 & 0.50 & 0.50 & & \\
\hline Burn-leach (DRF-26B) $(\mu \mathrm{g})$ : & 0.20 & 0.20 & 0.20 & 0.20 & 0.19 & 0.19 & 0.19 & 0.20 & & \\
\hline Total leached $(\mu \mathrm{g})$ : & 0.69 & 0.69 & 0.70 & 0.65 & 0.69 & 0.70 & 0.69 & 0.70 & & \\
\hline Mn outside SiC ( $\mu \mathrm{g} / \mathrm{compact}):$ & 0.14 & 0.14 & 0.14 & 0.13 & $\mathbf{0 . 1 4}$ & 0.14 & 0.14 & 0.14 & \multicolumn{2}{|c|}{ Continued on next page } \\
\hline \multicolumn{11}{|l|}{ Cobalt } \\
\hline Deconsolidation-leach (DRF-26A) $(\mu \mathrm{g})$ : & 0.42 & 0.41 & 0.43 & 0.38 & 0.42 & 0.43 & 0.42 & 0.43 & & \\
\hline Burn-leach (DRF-26B) $(\mu \mathrm{g}):$ & 0.17 & 0.17 & 0.17 & 0.17 & 0.16 & 0.16 & 0.16 & 0.17 & & \\
\hline Total leached $(\mu \mathrm{g})$ : & 0.59 & 0.58 & 0.60 & 0.55 & 0.59 & 0.60 & 0.58 & 0.59 & & \\
\hline Co outside SiC ( $\mu \mathrm{g} /$ compact): & 0.12 & 0.12 & 0.12 & $\mathbf{0 . 1 1}$ & 0.12 & 0.12 & 0.12 & 0.12 & \multicolumn{2}{|c|}{ Continued on next page } \\
\hline \multicolumn{11}{|l|}{ Nickel } \\
\hline Deconsolidation-leach (DRF-26A) $(\mu \mathrm{g})$ : & 2.08 & 2.51 & 2.12 & 1.98 & 2.09 & 2.13 & 2.47 & 2.10 & & \\
\hline Burn-leach (DRF-26B) $(\mu \mathrm{g})$ : & 2.43 & 4.18 & 1.18 & 2.42 & 2.96 & 2.51 & 3.17 & 3.60 & & \\
\hline Total leached $(\mu \mathrm{g})$ : & 4.51 & 6.69 & 3.30 & 4.40 & 5.05 & 4.64 & 5.64 & 5.70 & & \\
\hline Ni outside SiC ( $\mu \mathrm{g} /$ compact): & 0.90 & 1.34 & 0.66 & 0.88 & 1.01 & 0.93 & 1.13 & 1.14 & \multicolumn{2}{|c|}{ Continued on next page } \\
\hline \multicolumn{11}{|l|}{ Transition Metals } \\
\hline $\mathrm{Cr}+\mathrm{Mn}+\mathrm{Co}+\mathrm{Ni}$ outside $\mathrm{SiC}(\mu \mathrm{g} /$ compact): & 1.70 & 2.07 & 1.61 & 1.72 & 1.78 & 1.82 & 1.84 & 1.80 & \multicolumn{2}{|c|}{ Continued on next page } \\
\hline \multicolumn{11}{|l|}{ Calcium } \\
\hline Deconsolidation-leach (DRF-26A) $(\mu \mathrm{g})$ : & 26.00 & 38.52 & 78.49 & 54.45 & 0.00 & 117.86 & 5.36 & 273.96 & & \\
\hline Burn-leach (DRF-26B) $(\mu \mathrm{g})$ : & 118.60 & 190.06 & 108.08 & 155.96 & 119.43 & 98.12 & 94.92 & 103.55 & & \\
\hline Total leached $(\mu \mathrm{g}):$ & 144.60 & 228.58 & 186.57 & 210.41 & 119.43 & 215.98 & 100.28 & 377.51 & & \\
\hline Ca outside $\mathrm{SiC}(\mu \mathrm{g} /$ compact $):$ & 28.92 & 45.72 & 37.31 & 42.08 & 23.89 & 43.20 & 20.06 & 75.50 & \multicolumn{2}{|c|}{ Continued on next page } \\
\hline \multicolumn{11}{|l|}{ Aluminum } \\
\hline Deconsolidation-leach (DRF-26A) $(\mu \mathrm{g})$ : & 67.76 & 53.78 & 64.21 & 71.11 & 55.71 & 73.83 & 55.95 & 51.47 & & \\
\hline Burn-leach (DRF-26B) $(\mu \mathrm{g})$ : & 78.80 & 78.86 & 78.30 & 76.42 & 82.43 & 90.36 & 82.05 & 84.30 & & \\
\hline Total leached $(\mu \mathrm{g}):$ & 146.56 & 132.65 & 142.50 & 147.53 & 138.14 & 164.19 & 138.01 & 135.76 & & \\
\hline Al outside $\mathrm{SiC}(\mu \mathrm{g} /$ compact $)$ : & 29.31 & 26.53 & 28.50 & 29.51 & 27.63 & 32.84 & 27.60 & 27.15 & \multicolumn{2}{|c|}{ Continued on next page } \\
\hline \multicolumn{11}{|l|}{ Titanium } \\
\hline Deconsolidation-leach (DRF-26A) $(\mu \mathrm{g})$ : & 5.58 & 5.20 & 11.19 & 4.78 & 5.65 & 6.83 & 4.02 & 4.95 & & \\
\hline Burn-leach (DRF-26B) $(\mu \mathrm{g})$ : & 7.76 & 8.55 & 5.06 & 7.39 & 8.26 & 7.49 & 9.11 & 9.39 & & \\
\hline Total leached $(\mu \mathrm{g}):$ & 13.34 & 13.75 & 16.25 & 12.17 & 13.91 & 14.32 & 13.13 & 14.34 & & \\
\hline Ti outside $\mathrm{SiC}(\mu \mathrm{g} /$ compact $):$ & 2.67 & 2.75 & 3.25 & 2.43 & 2.78 & 2.86 & 2.63 & 2.87 & \multicolumn{2}{|c|}{ Continued on next page } \\
\hline Vanadium & & & & & & & & & & \\
\hline Deconsolidation-leach (DRF-26A) $(\mu \mathrm{g})$ : & 40.04 & 40.62 & 40.32 & 40.09 & 34.12 & 32.55 & 31.85 & 30.10 & & \\
\hline Burn-leach (DRF-26B) $(\mu \mathrm{g})$ : & 54.39 & 61.01 & 51.42 & 53.84 & 58.38 & 11.67 & 57.59 & 58.85 & & \\
\hline Total leached $(\mu \mathrm{g})$ : & 94.43 & 101.63 & 91.73 & 93.94 & 92.50 & 44.22 & 89.44 & 88.95 & & \\
\hline V outside $\mathrm{SiC}(\mu \mathrm{g} /$ compact $):$ & 18.89 & 20.33 & 18.35 & 18.79 & 18.50 & 8.84 & 17.89 & 17.79 & Continu & next page \\
\hline Titanium and Vanadium & & & & & & & & & & \\
\hline $\mathrm{Ti}+\mathrm{V}$ outside $\mathrm{SiC}(\mu \mathrm{g} /$ compact $)$ : & 21.55 & 23.08 & 21.60 & 21.22 & 21.28 & 11.71 & 20.51 & 20.66 & Continu & next pag \\
\hline
\end{tabular}


Table 7-1: Summary of impurity analysis for LEU09-OP2-Z compacts (continued)

\begin{tabular}{|c|c|c|c|c|c|c|c|c|c|c|}
\hline Compact ID numbers: & $\begin{array}{c}050,044, \\
020,164, \\
098\end{array}$ & $\begin{array}{c}027,157 \\
054,109 \\
070\end{array}$ & $\begin{array}{c}025,106 \\
133,110 \\
029\end{array}$ & $\begin{array}{c}113,162, \\
055,137 \\
123\end{array}$ & $\begin{array}{c}108,006 \\
096,073, \\
094\end{array}$ & $\begin{array}{c}166,111, \\
099,042, \\
120\end{array}$ & $\begin{array}{c}101,078, \\
136,087, \\
122\end{array}$ & $\begin{array}{c}052,146 \\
161,115, \\
095\end{array}$ & Mean & $\begin{array}{c}\text { Standard } \\
\text { Deviation }\end{array}$ \\
\hline Number of compacts: & $\overline{5}$ & $\overline{5}$ & $\overline{5}$ & $\overline{5}$ & $\overline{5}$ & $\overline{5}$ & $\overline{5}$ & $\overline{5}$ & & \\
\hline \multicolumn{11}{|l|}{ Iron } \\
\hline Deconsolidation-leach (DRF-26A) $(\mu \mathrm{g})$ : & 10.46 & 10.05 & 10.46 & 10.46 & 9.81 & 10.26 & 10.01 & 10.18 & & \\
\hline Burn-leach (DRF-26B) $(\mu \mathrm{g})$ : & 5.25 & 4.83 & 5.25 & 5.21 & 7.09 & 6.87 & 4.85 & 5.14 & & \\
\hline Total leached $(\mu \mathrm{g})$ : & 15.72 & 14.88 & 15.72 & 15.68 & 16.89 & 17.13 & 14.86 & 15.32 & & \\
\hline Fe outside $\mathrm{SiC}(\mu \mathrm{g} /$ compact $):$ & 3.14 & 2.98 & 3.14 & 3.14 & 3.38 & 3.43 & 2.97 & 3.06 & 4.04 & 2.52 \\
\hline \multicolumn{11}{|l|}{ Chromium } \\
\hline Deconsolidation-leach (DRF-26A) $(\mu \mathrm{g})$ : & 1.76 & 2.10 & 1.53 & 1.79 & 2.77 & 2.73 & 2.61 & 2.74 & & \\
\hline Burn-leach (DRF-26B) $(\mu \mathrm{g})$ : & 1.18 & 0.98 & 1.23 & 1.09 & 1.29 & 1.10 & 1.09 & 1.20 & & \\
\hline Total leached $(\mu \mathrm{g})$ : & 2.95 & 3.07 & 2.76 & 2.88 & 4.06 & 3.83 & 3.70 & 3.94 & & \\
\hline Cr outside SiC ( $\mu \mathrm{g} /$ compact): & $\mathbf{0 . 5 9}$ & 0.61 & 0.55 & 0.58 & 0.81 & $\mathbf{0 . 7 7}$ & $\mathbf{0 . 7 4}$ & $\mathbf{0 . 7 9}$ & 0.61 & 0.12 \\
\hline \multicolumn{11}{|l|}{ Manganese } \\
\hline Deconsolidation-leach (DRF-26A) $(\mu \mathrm{g})$ : & 0.49 & 0.47 & 0.49 & 0.49 & 0.45 & 0.48 & 0.46 & 0.47 & & \\
\hline Burn-leach (DRF-26B) $(\mu \mathrm{g})$ : & 0.20 & 0.18 & 0.19 & 0.20 & 0.20 & 0.19 & 0.19 & 0.20 & & \\
\hline Total leached $(\mu \mathrm{g})$ : & 0.68 & 0.65 & 0.68 & 0.68 & 0.65 & 0.67 & 0.65 & 0.67 & & \\
\hline Mn outside $\mathrm{SiC}(\mu \mathrm{g} /$ compact $)$ : & 0.14 & 0.13 & 0.14 & 0.14 & 0.13 & 0.13 & 0.13 & 0.13 & 0.136 & 0.004 \\
\hline \multicolumn{11}{|l|}{ Cobalt } \\
\hline Deconsolidation-leach (DRF-26A) $(\mu \mathrm{g})$ : & 0.41 & 0.40 & 0.41 & 0.41 & 0.39 & 0.40 & 0.39 & 0.40 & & \\
\hline Burn-leach (DRF-26B) $(\mu \mathrm{g})$ : & 0.17 & 0.15 & 0.16 & 0.17 & 0.17 & 0.16 & 0.16 & 0.17 & & \\
\hline Total leached $(\mu \mathrm{g})$ : & 0.58 & 0.55 & 0.58 & 0.58 & 0.55 & 0.57 & 0.55 & 0.57 & & \\
\hline Co outside $\mathrm{SiC}(\mu \mathrm{g} / \mathrm{compact})$ : & 0.12 & 0.11 & 0.12 & 0.12 & 0.11 & 0.11 & 0.11 & 0.11 & 0.115 & 0.003 \\
\hline \multicolumn{11}{|l|}{ Nickel } \\
\hline Deconsolidation-leach (DRF-26A) $(\mu \mathrm{g})$ : & 2.03 & 1.95 & 2.03 & 2.03 & 2.56 & 1.99 & 1.94 & 1.98 & & \\
\hline Burn-leach (DRF-26B) $(\mu \mathrm{g})$ : & 3.12 & 2.32 & 2.00 & 3.07 & 4.11 & 1.94 & 1.83 & 2.24 & & \\
\hline Total leached $(\mu \mathrm{g})$ : & 5.15 & 4.27 & 4.04 & 5.10 & 6.67 & 3.93 & 3.78 & 4.22 & & \\
\hline Ni outside $\mathrm{SiC}(\mu \mathrm{g} /$ compact $)$ : & 1.03 & 0.85 & 0.81 & 1.02 & 1.33 & 0.79 & 0.76 & 0.84 & 0.96 & 0.20 \\
\hline \multicolumn{11}{|l|}{ Transition Metals } \\
\hline $\mathrm{Cr}+\mathrm{Mn}+\mathrm{Co}+\mathrm{Ni}$ outside $\mathrm{SiC}(\mu \mathrm{g} /$ compact $):$ & 1.87 & 1.71 & 1.61 & 1.85 & 2.39 & 1.80 & 1.74 & 1.88 & 1.82 & 0.19 \\
\hline \multicolumn{11}{|l|}{ Calcium } \\
\hline Deconsolidation-leach (DRF-26A) $(\mu \mathrm{g})$ : & 88.60 & 85.37 & 95.35 & 31.87 & 65.37 & 44.56 & 93.82 & 131.76 & & \\
\hline Burn-leach (DRF-26B) $(\mu \mathrm{g})$ : & 118.79 & 91.28 & 107.44 & 110.55 & 133.78 & 132.53 & 106.42 & 126.27 & & \\
\hline Total leached $(\mu \mathrm{g})$ : & 207.39 & 176.65 & 202.79 & 142.41 & 199.15 & 177.08 & 200.25 & 258.03 & & \\
\hline Ca outside $\mathrm{SiC}(\mu \mathrm{g} /$ compact): & 41.48 & 35.33 & 40.56 & 28.48 & 39.83 & $\mathbf{3 5 . 4 2}$ & 40.05 & 51.61 & 39.34 & 12.65 \\
\hline \multicolumn{11}{|l|}{ Aluminum } \\
\hline Deconsolidation-leach (DRF-26A) $(\mu \mathrm{g})$ : & 63.46 & 69.42 & 50.61 & 65.89 & 96.66 & 66.76 & 60.57 & 70.30 & & \\
\hline Burn-leach (DRF-26B) $(\mu \mathrm{g})$ : & 79.76 & 67.14 & 76.53 & 78.55 & 102.16 & 86.67 & 91.71 & 96.17 & & \\
\hline Total leached $(\mu \mathrm{g})$ : & 143.21 & 136.56 & 127.14 & 144.44 & 198.81 & 153.43 & 152.28 & 166.47 & & \\
\hline Al outside $\mathrm{SiC}(\mu \mathrm{g} /$ compact $):$ & 28.64 & 27.31 & 25.43 & 28.89 & 39.76 & 30.69 & 30.46 & 33.29 & 29.60 & 3.44 \\
\hline \multicolumn{11}{|l|}{ Titanium } \\
\hline Deconsolidation-leach (DRF-26A) $(\mu \mathrm{g})$ : & 6.27 & 7.28 & 6.69 & 4.36 & 8.23 & 8.10 & 8.10 & 8.39 & & \\
\hline Burn-leach (DRF-26B) $(\mu \mathrm{g})$ : & 7.95 & 7.03 & 6.81 & 8.68 & 6.56 & 6.24 & 7.44 & 5.86 & & \\
\hline Total leached $(\mu \mathrm{g})$ : & 14.22 & 14.30 & 13.50 & 13.04 & 14.79 & 14.34 & 15.54 & 14.25 & & \\
\hline Ti outside $\mathrm{SiC}(\mu \mathrm{g} /$ compact $):$ & 2.84 & 2.86 & 2.70 & 2.61 & 2.96 & 2.87 & 3.11 & 2.85 & 2.81 & 0.20 \\
\hline \multicolumn{11}{|l|}{ Vanadium } \\
\hline Deconsolidation-leach (DRF-26A) $(\mu \mathrm{g})$ : & 29.70 & 33.85 & 27.04 & 29.20 & 33.76 & 34.55 & 34.68 & 32.27 & & \\
\hline Burn-leach (DRF-26B) $(\mu \mathrm{g})$ : & 52.91 & 46.51 & 55.06 & 52.70 & 53.62 & 50.93 & 54.19 & 49.51 & & \\
\hline Total leached $(\mu \mathrm{g})$ : & 82.61 & 80.36 & 82.10 & 81.90 & 87.38 & 85.47 & 88.88 & 81.78 & & \\
\hline V outside $\mathrm{SiC}(\mu \mathrm{g} /$ compact $)$ : & 16.52 & 16.07 & 16.42 & 16.38 & 17.48 & 17.09 & $\mathbf{1 7 . 7 8}$ & 16.36 & 17.09 & 2.49 \\
\hline \multicolumn{11}{|l|}{ Titanium and Vanadium } \\
\hline $\mathrm{Ti}+\mathrm{V}$ outside SiC ( $\mu \mathrm{g} /$ compact $):$ & 19.37 & 18.93 & 19.12 & 18.99 & 20.43 & 19.96 & 20.88 & 19.21 & 19.91 & 2.47 \\
\hline
\end{tabular}


Table 7-2: Summary of uranium contamination and SiC defect analysis for LEU09-OP2-Z compacts

\begin{tabular}{|c|c|c|c|}
\hline Compact ID numbers & Number of compacts & $\begin{array}{c}\text { Effective number of exposed } \\
\text { kernels before burn }\end{array}$ & $\begin{array}{c}\text { Number of kernels } \\
\text { leached after burn }\end{array}$ \\
\hline \hline $011,152,001,068,046$ & 5 & 0.0 & 0 \\
\hline $067,081,019,008,032$ & 5 & 0.0 & 0 \\
\hline $039,036,155,012,117$ & 5 & 0.0 & 0 \\
\hline $031,149,093,147,072$ & 5 & 0.0 & 0 \\
\hline $051,086,143,097,033$ & 5 & 0.0 & 0 \\
\hline $030,024,090,013,010$ & 5 & 0.0 & 0 \\
\hline $021,035,121,107,061$ & 5 & 0.9 & 0 \\
\hline $151,003,058,080,009$ & 5 & 0.0 & Not analyzed \\
\hline $118,041,138,007,071$ & 5 & 0.0 & Not analyzed \\
\hline $144,048,023,037,047$ & 5 & 0.0 & Not analyzed \\
\hline $150,065,116,140,160$ & 5 & 0.8 & 0 \\
\hline $034,084,074,159,060$ & 5 & 0.0 & 0 \\
\hline $050,044,020,164,098$ & 5 & 0.0 & 0 \\
\hline $027,157,054,109,070$ & 5 & 0.9 & 0 \\
\hline $025,106,133,110,029$ & 5 & 0.0 & 0 \\
\hline $113,162,055,137,123$ & 5 & 0.0 & 0 \\
\hline $108,006,096,073,094$ & 5 & 0.0 & 0 \\
\hline $166,111,099,042,120$ & 5 & 0.0 & 0 \\
\hline $101,078,136,087,122$ & 5 & 0.0 & $\mathbf{0}$ \\
\hline $052,146,161,115,095$ & 5 & 0.0 & $\mathbf{3 . 1}$ \\
\hline \hline Total: & $\mathbf{1 0 0}$ & & \\
\hline
\end{tabular}

After compacts were electrolytically deconsolidated and leached, uranium was detected at a level equivalent to $\sim 3$ kernels out of the $\sim 317625$ particles leached. This corresponds to a binomial distribution defect fraction of $\leq 2.5 \mathrm{E}-5$ at $95 \%$ confidence, which is above the specified limit of $\leq 2.0 \mathrm{E}-5$. Analysis of as-coated TRISO particles from the same batch (G73J-14-93073A) showed a similar defect fraction. Further analysis determined that the source of the defects was cracked TRISO coatings on a small fraction of otherwise normal particles. This damage is thought to have occurred at $\mathrm{B} \& \mathrm{~W}$ during removal of the particles from the coating furnace via a suction transfer system. Because the suspected root cause of this nonconformance was not related to the compacting and characterization activities at ORNL, a nonconformance report was issued by INL (NCR-44791), with a disposition to "use as is" for the AGR-2 irradiation test.

The measured exposed uranium defect fraction for the 100 compact sample was 3 out of 317,625 particles, which is less than 1E-5. This indicates that the entire compact lot may have a defect fraction below the specified limit. However, it would require a greater number of compacts be analyzed in order to verify this at $95 \%$ confidence. Without additional compact analysis, the exposed uranium defect fraction for the entire compact lot can only be determined to be below the $2 \mathrm{E}-5$ specified limit to $85 \%$ confidence using binomial distribution statistics. One could also consider the use of a hypergeometric distribution to calculate the exposed uranium defect fraction. The binomial distribution (specified by PIP-12 for acceptance test calculations) is commonly used for coated particle fuel analysis and is more conservative than the hypergeometric distribution. However, when the sample size is a significant fraction of the entire population, which is the case for this analysis, it is more appropriate to apply the hypergeometric 
distribution. The hypergeometric distribution would predict a defect fraction of $\leq 2.1 \mathrm{E}-5$ at $95 \%$ confidence, based on the measured sample. Alternately, the hypergeometric distribution would determine the uranium contamination defect fraction to be below the $2 \mathrm{E}-5$ specified limit to 94.4\% confidence. Regardless of the chosen calculation method, it is obvious that these compacts are, at worse, only marginally over the specified limit for exposed uranium. 
ORNL/TM-2010/017

Inspection Report Form IRF-12A: AGR-2 UCO Fuel Compact Lots

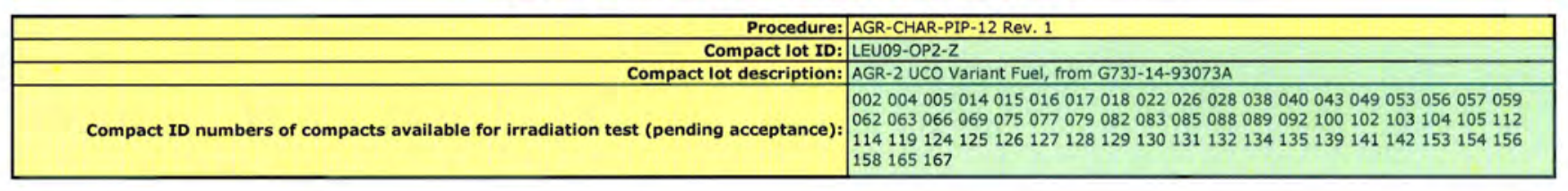

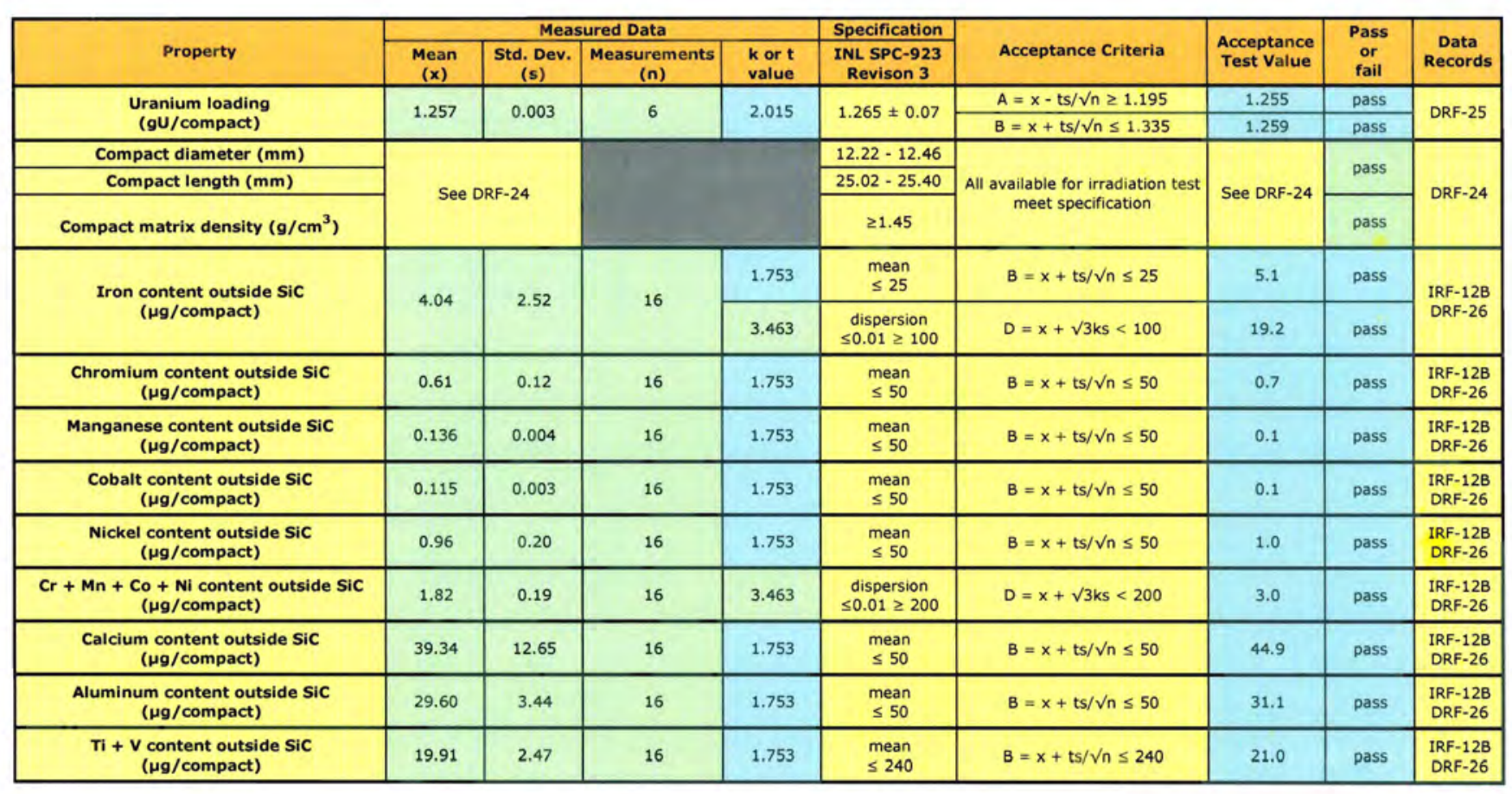

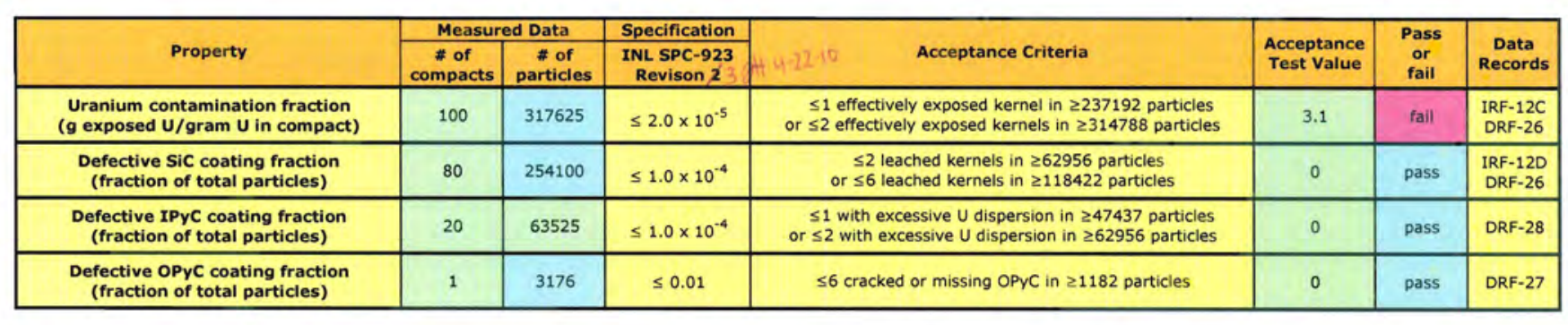

Comments

A $3 / 317625$ uranium contamination fraction is above the specified limit and corresponds to $<2.5 \mathrm{e}-5$ at $95 \%$ confidence. This non-conformance was documented on INL NCR-44791 with a disposition of use as is.

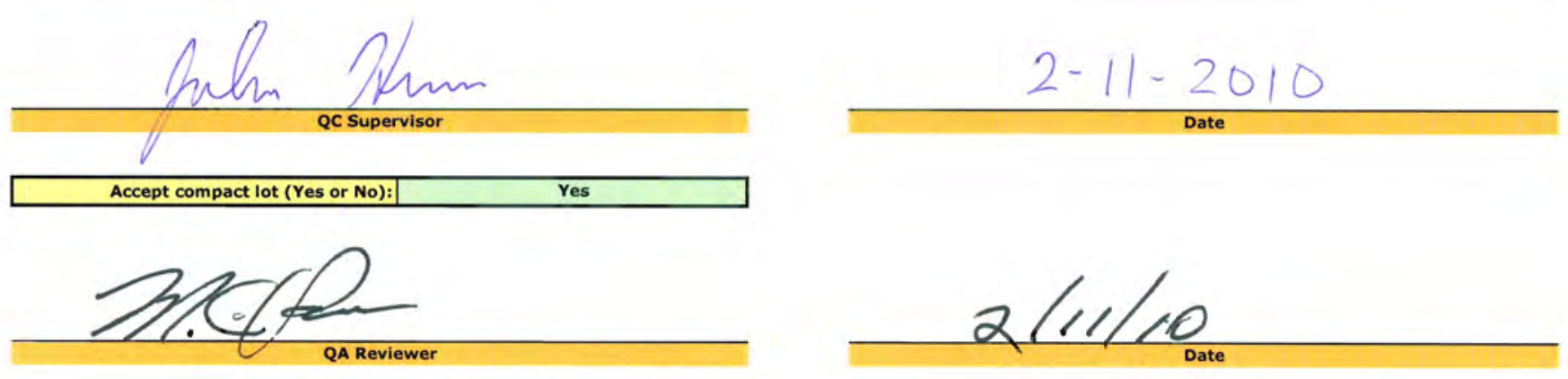

45 
Inspection Report Form IRF-12B: Summary of Impurites Outside SIC - Maximum Corrected Values

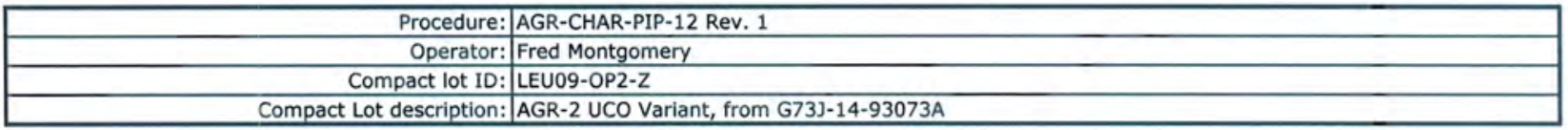

\begin{tabular}{|c|c|c|c|c|c|c|}
\hline Compact ID numbers: & $\begin{array}{c}011,152,001 \\
068,046 \\
\end{array}$ & $\begin{array}{c}067,081,019 \\
008,032 \\
\end{array}$ & $\begin{array}{c}039,036,155 \\
012,117 \\
\end{array}$ & $\begin{array}{c}031,149,093 \\
147,072 \\
\end{array}$ & Mean & $\begin{array}{l}\text { Standard } \\
\text { Deviation } \\
\end{array}$ \\
\hline Number of compacts: & 5 & 5 & 5 & 5 & & \\
\hline \multicolumn{7}{|l|}{ 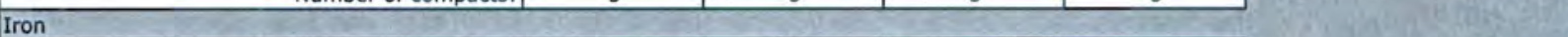 } \\
\hline Deconsolidation-leach (DRF-26A) $(\mu \mathrm{g})$ : & 10.71 & 10.55 & 10.92 & 9.76 & & \\
\hline Burn-leach (DRF-26B) $(\mu \mathrm{g})$ : & 6.17 & 24.50 & 5.11 & 5.24 & & \\
\hline Total leached $(\mu g):$ & 16.88 & 35.04 & 16.03 & 15.01 & & \\
\hline Fe outside SiC ( $\mu \mathrm{g} /$ compact): & 3.38 & 7.01 & 3.21 & 3.00 & 4.15 & 1.91 \\
\hline \multicolumn{7}{|l|}{ Chromium } \\
\hline Deconsolidation-leach (DRF-26A) ( $\mu \mathrm{g})$ : & 1.45 & 1.14 & 2.30 & 1.85 & & \\
\hline Burn-leach (DRF-26B) $(\mu \mathrm{g})$ : & 1.26 & 1.24 & 1.16 & 1.15 & & \\
\hline Total leached $(\mu g)$ : & 2.71 & 2.38 & 3.46 & 3.00 & & \\
\hline Cr outside $\operatorname{SiC}(\mu \mathrm{g} /$ compact): & 0.54 & 0.48 & 0.69 & 0.60 & 0.58 & 0.09 \\
\hline \multicolumn{5}{|l|}{ Manganese } & & \\
\hline Deconsolidation-leach (DRF-26A) $(\mu \mathrm{g}):$ & 0.50 & 0.49 & 0.51 & 0.45 & & \\
\hline Burn-leach (DRF-26B) $(\mu \mathrm{g})$ : & 0.20 & 0.20 & 0.20 & 0.20 & & \\
\hline Total leached $(\mu g)$ : & 0.69 & 0.69 & 0.70 & 0.65 & & \\
\hline Mn outside SiC $(\mu \mathrm{g} /$ compact $):$ & 0.14 & 0.14 & 0.14 & 0.13 & 0.137 & 0.004 \\
\hline \multicolumn{7}{|l|}{ Cobalt } \\
\hline Deconsolidation-leach (DRF-26A) $(\mu \mathrm{g}):$ & 0.42 & 0.41 & 0.43 & 0.38 & & \\
\hline Burn-leach (DRF-26B) $(\mu \mathrm{g}):$ & 0.17 & 0.17 & 0.17 & 0.17 & & \\
\hline Total leached $(\mu \mathrm{g}):$ & 0.59 & 0.58 & 0.60 & 0.55 & & \\
\hline Co outside SiC ( $\mu \mathrm{g} /$ compact): & 0.12 & 0.12 & 0.12 & 0.11 & 0.116 & 0.004 \\
\hline \multicolumn{5}{|l|}{$\begin{aligned} 0 \\
\end{aligned}$} & & \\
\hline Deconsolidation-leach (DRF-26A) $(\mu \mathrm{g}):$ & 2.08 & 2.51 & 2.12 & 1.98 & & \\
\hline Burn-leach (DRF-26B) $(\mu \mathrm{g}):$ & 2.43 & 4.18 & 1.18 & 2.42 & & \\
\hline Total leached $(\mu g)$ : & 4.51 & 6.69 & 3.30 & 4.40 & & \\
\hline Ni outside SiC ( $\mu \mathrm{g} /$ compact): & 0.90 & 1.34 & 0.66 & 0.88 & 0.94 & 0.28 \\
\hline \multicolumn{7}{|l|}{ Transition Metals } \\
\hline $\mathrm{Cr}+\mathrm{Mn}+\mathrm{Co}+\mathrm{Ni}$ outside SiC ( $\mu \mathrm{g} /$ compact $):$ & 1.70 & 2.07 & 1.61 & 1.72 & 1.77 & 0.20 \\
\hline \multicolumn{7}{|l|}{ Calcium } \\
\hline Deconsolidation-leach (DRF-26A) $(\mu \mathrm{g}):$ & 26.00 & 38.52 & 78.49 & 54.45 & & \\
\hline Burn-leach (DRF-26B) $(\mu \mathrm{g}):$ & 118.60 & 190.06 & 108.08 & 155.96 & & \\
\hline Total leached $(\mu \mathrm{g})$ : & 144.60 & 228.58 & 186.57 & 210.41 & & \\
\hline Ca outside SiC ( $\mu \mathrm{g} /$ compact): & 28.92 & 45.72 & 37.31 & 42.08 & 38.51 & 7.26 \\
\hline \multicolumn{7}{|l|}{ Aluminum } \\
\hline Deconsolidation-leach (DRF-26A) $(\mu g)$ : & 67.76 & 53.78 & 64.21 & 71.11 & & \\
\hline Burn-leach (DRF-26B) $(\mu g)$ : & 78.80 & 78.86 & 78.30 & 76.42 & & \\
\hline Total leached $(\mu \mathrm{g})$ : & 146.56 & 132.65 & 142.50 & 147.53 & & \\
\hline Al outside $\operatorname{SiC}(\mu \mathrm{g} /$ compact): & 29.31 & 26.53 & 28.50 & 29.51 & 28.46 & 1.36 \\
\hline \multicolumn{7}{|l|}{ Titanium } \\
\hline Deconsolidation-leach (DRF-26A) $(\mu \mathrm{g})$ : & 5.58 & 5.20 & 11.19 & 4.78 & & \\
\hline Burn-leach (DRF-26B) $(\mu \mathrm{g})$ : & 7.76 & 8.55 & 5.06 & 7.39 & & \\
\hline Total leached $(\mu \mathrm{g})$ : & 13.34 & 13.75 & 16.25 & 12.17 & & \\
\hline Ti outside SiC ( $\mu \mathrm{g} /$ compact): & 2.67 & 2.75 & 3.25 & 2.43 & 2.78 & 0.34 \\
\hline \multicolumn{7}{|l|}{ Vanadium } \\
\hline Deconsolidation-leach (DRF-26A) $(\mu \mathrm{g})$ : & 40.04 & 40.62 & 40.32 & 40.09 & & \\
\hline Burn-leach (DRF-26B) $(\mu \mathrm{g})$ : & 54.39 & 61.01 & 51.42 & 53.84 & & \\
\hline Total leached $(\mu \mathrm{g}):$ & 94.43 & 101.63 & 91.73 & 93.94 & & \\
\hline V outside SiC ( $\mu \mathrm{g} /$ compact): & 18.89 & 20.33 & 18.35 & 18.79 & 19.09 & 0.86 \\
\hline \multicolumn{7}{|l|}{ Titanium and Vanadium } \\
\hline $\mathrm{Ti}+\mathrm{V}$ outside $\mathrm{SiC}(\mu \mathrm{g} /$ compact $):$ & 21.55 & 23.08 & 21.60 & 21.22 & 21.86 & 0.83 \\
\hline
\end{tabular}

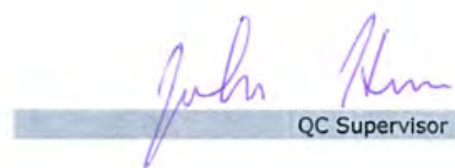


Inspection Report Form IRF-12B: Summary of Impurites Outside SiC - Maximum Corrected Values

\begin{tabular}{|r|l|}
\hline Procedure: & AGR-CHAR-PIP-12 Rev. 1 \\
\hline Operator: & Fred Montgomery \\
\hline Compact lot ID: & LEU09-OP2-Z \\
\hline Compact Lot description: & AGR-2 UCO Variant, from G73J-14-93073A \\
\hline
\end{tabular}

\begin{tabular}{|c|c|c|c|c|c|c|}
\hline Compact ID numbers: & $\begin{array}{c}051,086,143 \\
097,033 \\
\end{array}$ & $\begin{array}{c}030,024,090 \\
013,010\end{array}$ & $\begin{array}{c}021,035,121, \\
107,061\end{array}$ & $\begin{array}{c}151,003,058 \\
080,009\end{array}$ & Mean & $\begin{array}{l}\text { Standard } \\
\text { Deviation }\end{array}$ \\
\hline Number of compacts: & 5 & 5 & 5 & 5 & & \\
\hline \multicolumn{5}{|l|}{ Iron } & & \\
\hline Deconsolidation-leach (DRF-26A) ( $\mu g)$ : & 10.75 & 10.96 & 58.59 & 10.84 & & \\
\hline Burn-leach (DRF-26B) $(\mu \mathrm{g}):$ & 5.39 & 7.11 & 5.22 & 5.58 & & \\
\hline Total leached $(\mu \mathrm{g}):$ & 16.15 & 18.07 & 63.81 & 16.42 & & \\
\hline Fe outside SiC ( $\mu \mathrm{g} /$ compact): & 3.23 & 3.61 & 12.76 & 3.28 & 5.72 & 4.70 \\
\hline \multicolumn{5}{|l|}{ Chromium } & & \\
\hline Deconsolidation-leach (DRF-26A) $(\mu \mathrm{g})$ : & 1.33 & 1.90 & 1.10 & 0.71 & & \\
\hline Burn-leach (DRF-26B) $(\mu \mathrm{g})$ : & 1.25 & 1.29 & 1.18 & 1.28 & & \\
\hline Total leached $(\mu \mathrm{g}):$ & 2.58 & 3.19 & 2.27 & 1.99 & & \\
\hline Cr outside SiC ( $\mu \mathrm{g} /$ compact): & 0.52 & 0.64 & 0.45 & 0.40 & 0.50 & 0.10 \\
\hline \multicolumn{5}{|l|}{ Manganese } & & \\
\hline Deconsolidation-leach (DRF-26A) $(\mu \mathrm{g})$ : & 0.50 & 0.51 & 0.50 & 0.50 & & \\
\hline Burn-leach (DRF-26B) $(\mu \mathrm{g})$ : & 0.19 & 0.19 & 0.19 & 0.20 & & \\
\hline Total leached $(\mu \mathrm{g})$ : & 0.69 & 0.70 & 0.69 & 0.70 & & \\
\hline Mn outside SiC ( $\mu \mathrm{g} /$ compact): & 0.14 & 0.14 & 0.14 & 0.14 & 0.139 & 0.001 \\
\hline \multicolumn{5}{|l|}{ Cobalt } & & \\
\hline Deconsolidation-leach (DRF-26A) $(\mu \mathrm{g})$ : & 0.42 & 0.43 & 0.42 & 0.43 & & \\
\hline Burn-leach (DRF-26B) $(\mu \mathrm{g})$ : & 0.16 & 0.16 & 0.16 & 0.17 & & \\
\hline Total leached $(\mu \mathrm{g})$ : & 0.59 & 0.60 & 0.58 & 0.59 & & \\
\hline Co outside SiC ( $\mu \mathrm{g} /$ compact): & 0.12 & 0.12 & 0.12 & 0.12 & 0.118 & 0.001 \\
\hline \multicolumn{5}{|l|}{ Nickel } & & \\
\hline Deconsolidation-leach (DRF-26A) $(\mu g)$ : & 2.09 & 2.13 & 2.47 & 2.10 & & \\
\hline Burn-leach (DRF-26B) $(\mu \mathrm{g})$ : & 2.96 & 2.51 & 3.17 & 3.60 & & \\
\hline Total leached $(\mu g):$ & 5.05 & 4.64 & 5.64 & 5.70 & & \\
\hline Ni outside SiC ( $\mu \mathrm{g} /$ compact): & 1.01 & 0.93 & 1.13 & 1.14 & 1.05 & 0.10 \\
\hline \multicolumn{7}{|l|}{ Transition Metals } \\
\hline $\mathrm{Cr}+\mathrm{Mn}+\mathrm{Co}+\mathrm{Ni}$ outside SiC ( $\mu \mathrm{g} /$ compact): & 1.78 & 1.82 & 1.84 & 1.80 & 1.81 & 0.03 \\
\hline \multicolumn{5}{|l|}{ Calcium } & & \\
\hline Deconsolidation-leach (DRF-26A) $(\mu \mathrm{g})$ : & 0.00 & 117.86 & 5.36 & 273.96 & & \\
\hline Burn-leach (DRF-26B) $(\mu g)$ : & 119.43 & 98.12 & 94.92 & 103.55 & & \\
\hline Total leached $(\mu \mathrm{g}):$ & 119.43 & 215.98 & 100.28 & 377.51 & & \\
\hline Ca outside SiC ( $\mu \mathrm{g} /$ compact): & 23.89 & 43.20 & 20.06 & 75.50 & 40.66 & 25.34 \\
\hline \multicolumn{5}{|l|}{ Aluminum } & & \\
\hline Deconsolidation-leach (DRF-26A) $(\mu \mathrm{g})$ : & 55.71 & 73.83 & 55.95 & 51.47 & & \\
\hline Burn-leach (DRF-26B) $(\mu \mathrm{g})$ : & 82.43 & 90.36 & 82.05 & 84.30 & & \\
\hline Total leached $(\mu g)$ : & 138.14 & 164.19 & 138.01 & 135.76 & & \\
\hline Al outside SiC ( $\mu \mathrm{g} /$ compact): & 27.63 & 32.84 & 27.60 & 27.15 & 28.80 & 2.70 \\
\hline \multicolumn{5}{|l|}{ Titanium } & & \\
\hline Deconsolidation-leach (DRF-26A) ( $\mu \mathrm{g})$ : & 5.65 & 6.83 & 4.02 & 4.95 & & \\
\hline Burn-leach (DRF-26B) $(\mu \mathrm{g})$ : & 8.26 & 7.49 & 9.11 & 9.39 & & \\
\hline Total leached $(\mu \mathrm{g})$ : & 13.91 & 14.32 & 13.13 & 14.34 & & \\
\hline Ti outside SiC ( $\mu \mathrm{g} /$ compact): & 2.78 & 2.86 & 2.63 & 2.87 & 2.78 & 0.11 \\
\hline \multicolumn{5}{|l|}{ Vanadium } & & \\
\hline Deconsolidation-leach (DRF-26A) $(\mu g)$ : & 34.12 & 32.55 & 31.85 & 30.10 & & \\
\hline Burn-leach (DRF-26B) $(\mu \mathrm{g}):$ & 58.38 & 11.67 & 57.59 & 58.85 & & \\
\hline Total leached $(\mu \mathrm{g})$ : & 92.50 & 44.22 & 89.44 & 88.95 & & \\
\hline V outside SiC ( $\mu \mathrm{g} /$ compact): & 18.50 & 8.84 & 17.89 & 17.79 & 15.76 & 4.62 \\
\hline \multicolumn{7}{|l|}{ Titanium and Vanadium } \\
\hline$T i+V$ outside SiC $(\mu \mathrm{g} /$ compact $):$ & 21.28 & 11.71 & 20.51 & 20.66 & 18.54 & 4.57 \\
\hline
\end{tabular}

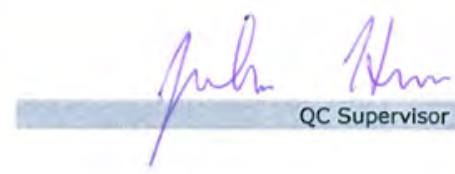


Inspection Report Form IRF-12B: Summary of Impurites Outside SiC - Maximum Corrected Values

\begin{tabular}{|r|l|}
\hline Procedure: & AGR-CHAR-PIP-12 Rev. 1 \\
\hline Operator: & Fred Montgomery \\
\hline Compact lot ID: & LEU09-OP2-Z \\
\hline Compact Lot description: & AGR-2 UCO Variant, from G73J-14-93073A \\
\hline
\end{tabular}

\begin{tabular}{|c|c|c|c|c|c|c|}
\hline Compact ID numbers: & $\begin{array}{c}118,041,138 \\
007,071\end{array}$ & $\begin{array}{c}144,048,023 \\
037,047\end{array}$ & $\begin{array}{c}150,065,116 \\
140,160 \\
\end{array}$ & $\begin{array}{c}034,084,074 \\
159,060\end{array}$ & Mean & $\begin{array}{l}\text { Standard } \\
\text { Deviation }\end{array}$ \\
\hline Number of compacts: & 5 & 5 & 5 & 5 & & \\
\hline \multicolumn{7}{|l|}{ (2) } \\
\hline Deconsolidation-leach (DRF-26A) $(\mu g)$ : & 9.64 & 11.12 & 10.75 & 10.30 & & \\
\hline \multicolumn{7}{|l|}{ Burn-leach (DRF-26B) $(\mu g)$ : } \\
\hline \multicolumn{7}{|l|}{ Total leached $(\mu \mathrm{g})$ : } \\
\hline \multicolumn{7}{|l|}{ Fe outside SiC $(\mu \mathrm{g} /$ compact): } \\
\hline Chromium & $2=$ & 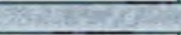 & 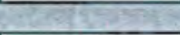 & 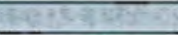 & & \\
\hline Deconsolidation-leach (DRF-26A) $(\mu \mathrm{g})$ : & 1.17 & 1.77 & 1.47 & 1.44 & & \\
\hline \multicolumn{7}{|l|}{ Burn-leach $(D R F-26 B)(\mu g)$ : } \\
\hline \multicolumn{7}{|l|}{$\begin{array}{r}\text { Total leached }(\mu g): \\
\end{array}$} \\
\hline \multicolumn{7}{|l|}{ Cr outside SiC ( $\mu \mathrm{g} /$ compact): } \\
\hline \multicolumn{7}{|l|}{ Manganese } \\
\hline Deconsolidation-leach (DRF-26A) $(\mu g)$ : & 0.45 & 0.48 & 0.50 & 0.48 & & \\
\hline \multicolumn{7}{|l|}{ Burn-leach (DRF-26B) $(\mu g)$ : } \\
\hline \multicolumn{7}{|l|}{ Total leached $(\mu \mathrm{g})$ : } \\
\hline \multirow{2}{*}{\multicolumn{7}{|c|}{ Mn outside SiC ( $\mu \mathrm{g} /$ compact): }} \\
\hline \multicolumn{6}{|l|}{ 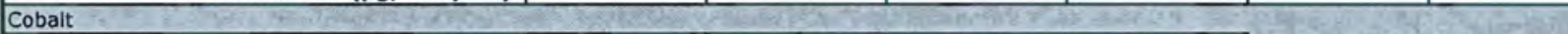 } & \\
\hline Deconsolidation-leach (DRF-26A) $(\mu \mathrm{g})$ : & 0.38 & 0.41 & 0.42 & 0.41 & & \\
\hline \multicolumn{7}{|l|}{ Burn-leach $(D R F-26 B)(\mu g)$ : } \\
\hline \multicolumn{7}{|l|}{ Total leached $(\mu \mathrm{g})$ : } \\
\hline \multicolumn{7}{|l|}{ Co outside SiC $(\mu \mathrm{g} /$ compact): } \\
\hline \multicolumn{7}{|l|}{ Nickel } \\
\hline Deconsolidation-leach (DRF-26A) $(\mu \mathrm{g})$ : & 1.87 & 2.02 & 2.09 & 2.00 & & \\
\hline \multicolumn{7}{|l|}{ Burn-leach (DRF-26B) $(\mu g)$ : } \\
\hline \multicolumn{7}{|l|}{ Total leached $(\mu \mathrm{g})$ : } \\
\hline \multirow{2}{*}{\multicolumn{7}{|c|}{$\begin{array}{l}\text { Ni outside SiC }(\mu \mathrm{g} / \text { compact }): \\
\text { Transition Metals }\end{array}$}} \\
\hline & & & & & & \\
\hline \multicolumn{7}{|l|}{$\mathrm{Cr}+\mathrm{Mn}+\mathrm{Co}+\mathrm{Ni}$ outside $\mathrm{SiC}(\mu \mathrm{g} /$ compact $):$} \\
\hline Calcium & +2 & & & $=1$ & & \\
\hline Deconsolidation-leach (DRF-26A) $(\mu \mathrm{g})$ : & 113.70 & 61.40 & 64.96 & 186.83 & & \\
\hline Burn-leach (DRF-26B) $(\mu \mathrm{g})$ : & & & & & & \\
\hline Total leached $(\mu \mathrm{g})$ : & & & & & & \\
\hline Ca outside SiC ( $\mu \mathrm{g} /$ compact): & & & & & & \\
\hline Aluminum & $=$ & 2 & $2=0$ & (3. & & \\
\hline Deconsolidation-leach (DRF-26A) $(\mu g)$ : & 68.32 & 64.43 & 70.41 & 74.53 & & \\
\hline Burn-leach (DRF-26B) $(\mu g)$ : & & & & & & \\
\hline Total leached $(\mu \mathrm{g})$ : & & & & & & \\
\hline Al outside SiC ( $\mu \mathrm{g} /$ compact): & & & & & & \\
\hline Titanium & & & & & & \\
\hline Deconsolidation-leach (DRF-26A) $(\mu g)$ : & 7.98 & 7.13 & 8.84 & 10.61 & & \\
\hline Burn-leach (DRF-26B) $(\mu g)$ : & & & & & & \\
\hline Total leached $(\mu \mathrm{g})$ : & & & & & & \\
\hline Ti outside SiC $(\mu \mathrm{g} /$ compact): & & & & & & \\
\hline Vanadium & & - & $\overline{5}$ & 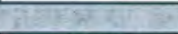 & & \\
\hline Deconsolidation-leach (DRF-26A) $(\mu g)$ : & 33.42 & 35.88 & 35.33 & 38.32 & & \\
\hline Burn-leach (DRF-26B) $(\mu g)$ : & & & & & & \\
\hline Total leached $(\mu \mathrm{g})$ : & & & & & & \\
\hline V outside $\operatorname{SiC}(\mu \mathrm{g} /$ compact): & & & & & & \\
\hline anium and Vanadium & & & & & & \\
\hline $\mathrm{Ti}+\mathrm{V}$ outside $\mathrm{SiC}(\mu \mathrm{g} /$ compact): & & & & & & \\
\hline
\end{tabular}

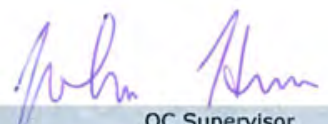


Inspection Report Form IRF-12B: Summary of Impurites Outside SiC - Maximum Corrected Values

\begin{tabular}{|r|l|}
\hline Procedure: & AGR-CHAR-PIP-12 Rev. 1 \\
\hline Operator: & Fred Montgomery \\
\hline Compact lot ID: & LEU09-OP2-Z \\
\hline Compact Lot description: & AGR-2 UCO Variant, from G73J-14-93073A \\
\hline
\end{tabular}

\begin{tabular}{|c|c|c|c|c|c|c|}
\hline Compact ID numbers: & $\begin{array}{c}050,044,020 \\
164,098\end{array}$ & $\begin{array}{c}027,157,054 \\
109,070\end{array}$ & $\begin{array}{c}025,106,133 \\
110,029 \\
\end{array}$ & $\begin{array}{c}113,162,055 \\
137,123 \\
\end{array}$ & Mean & $\begin{array}{l}\text { Standard } \\
\text { Deviation }\end{array}$ \\
\hline Number of compacts: & 5 & 5 & 5 & 5 & & \\
\hline \multicolumn{7}{|l|}{ Iron } \\
\hline Deconsolidation-leach (DRF-26A) $(\mu \mathrm{g})$ : & 10.46 & 10.05 & 10.46 & 10.46 & & \\
\hline Burn-leach (DRF-26B) $(\mu g)$ : & 5.25 & 4.83 & 5.25 & 5.21 & & \\
\hline Total leached $(\mu \mathrm{g}):$ & 15.72 & 14.88 & 15.72 & 15.68 & & \\
\hline Fe outside SiC ( $\mu g /$ compact): & 3.14 & 2.98 & 3.14 & 3.14 & 3.10 & 0.08 \\
\hline \multicolumn{5}{|l|}{ Chromium } & & \\
\hline Deconsolidation-leach (DRF-26A) ( $\mu \mathrm{g})$ : & 1.76 & 2.10 & 1.53 & 1.79 & & \\
\hline Burn-leach (DRF-26B) $(\mu g)$ : & 1.18 & 0.98 & 1.23 & 1.09 & & \\
\hline Total leached $(\mu g):$ & 2.95 & 3.07 & 2.76 & 2.88 & & \\
\hline Cr outside SiC ( $\mu \mathrm{g} /$ compact): & 0.59 & 0.61 & 0.55 & 0.58 & 0.58 & 0.03 \\
\hline \multicolumn{5}{|l|}{ Manganese } & & \\
\hline Deconsolidation-leach (DRF-26A) ( $\mu \mathrm{g})$ : & 0.49 & 0.47 & 0.49 & 0.49 & & \\
\hline Burn-leach (DRF-26B) $(\mu g)$ : & 0.20 & 0.18 & 0.19 & 0.20 & & \\
\hline Total leached $(\mu \mathrm{g}):$ & 0.68 & 0.65 & 0.68 & 0.68 & & \\
\hline Mn outside SiC ( $\mu \mathrm{g} /$ compact): & 0.14 & 0.13 & 0.14 & 0.14 & 0.134 & 0.004 \\
\hline \multicolumn{5}{|l|}{ 1 } & & \\
\hline Deconsolidation-leach (DRF-26A) $(\mu \mathrm{g})$ : & 0.41 & 0.40 & 0.41 & 0.41 & & \\
\hline Burn-leach (DRF-26B) $(\mu \mathrm{g})$ : & 0.17 & 0.15 & 0.16 & 0.17 & & \\
\hline Total leached $(\mu \mathrm{g}):$ & 0.58 & 0.55 & 0.58 & 0.58 & & \\
\hline Co outside SiC ( $\mu \mathrm{g} /$ compact): & 0.12 & 0.11 & 0.12 & 0.12 & 0.114 & 0.003 \\
\hline \multicolumn{5}{|l|}{ Nickel } & & \\
\hline Deconsolidation-leach (DRF-26A) ( $\mu \mathrm{g})$ : & 2.03 & 1.95 & 2.03 & 2.03 & & \\
\hline Burn-leach (DRF-26B) $(\mu \mathrm{g})$ : & 3.12 & 2.32 & 2.00 & 3.07 & & \\
\hline Total leached $(\mu \mathrm{g}):$ & 5.15 & 4.27 & 4.04 & 5.10 & & \\
\hline Ni outside SiC ( $\mu \mathrm{g} /$ compact): & 1.03 & 0.85 & 0.81 & 1.02 & 0.93 & 0.11 \\
\hline \multicolumn{7}{|l|}{ Transition Metals } \\
\hline $\mathrm{Cr}+\mathrm{Mn}+\mathrm{Co}+\mathrm{Ni}$ outside SiC ( $\mu \mathrm{g} /$ compact): & 1.87 & 1.71 & 1.61 & 1.85 & 1.76 & 0.12 \\
\hline \multicolumn{5}{|l|}{ Calcium } & & \\
\hline Deconsolidation-leach (DRF-26A) ( $\mu \mathrm{g})$ : & 88.60 & 85.37 & 95.35 & 31.87 & & \\
\hline Burn-leach (DRF-26B) $(\mu g)$ : & 118.79 & 91.28 & 107.44 & 110.55 & & \\
\hline Total leached $(\mu \mathrm{g}):$ & 207.39 & 176.65 & 202.79 & 142.41 & & \\
\hline Ca outside SiC ( $\mu \mathrm{g} /$ compact): & 41.48 & 35.33 & 40.56 & 28.48 & 36.46 & 5.97 \\
\hline \multicolumn{5}{|l|}{ Aluminum } & & \\
\hline Deconsolidation-leach (DRF-26A) $(\mu \mathrm{g})$ : & 63.46 & 69.42 & 50.61 & 65.89 & & \\
\hline Burn-leach (DRF-26B) $(\mu g)$ : & 79.76 & 67.14 & 76.53 & 78.55 & & \\
\hline Total leached $(\mu \mathrm{g})$ : & 143.21 & 136.56 & 127.14 & 144.44 & & \\
\hline Al outside SiC ( $\mu \mathrm{g} /$ compact): & 28.64 & 27.31 & 25.43 & 28.89 & 27.57 & 1.59 \\
\hline \multicolumn{5}{|l|}{ Titanium } & & \\
\hline Deconsolidation-leach (DRF-26A) $(\mu \mathrm{g})$ : & 6.27 & 7.28 & 6.69 & 4.36 & & \\
\hline Burn-leach (DRF-26B) $(\mu g)$ : & 7.95 & 7.03 & 6.81 & 8.68 & & \\
\hline Total leached $(\mu \mathrm{g}):$ & 14.22 & 14.30 & 13.50 & 13.04 & & \\
\hline Ti outside SiC ( $\mu \mathrm{g} /$ compact): & 2.84 & 2.86 & 2.70 & 2.61 & 2.75 & 0.12 \\
\hline \multicolumn{5}{|l|}{ Vanadium } & & \\
\hline Deconsolidation-leach (DRF-26A) $(\mu \mathrm{g})$ : & 29.70 & 33.85 & 27.04 & 29.20 & & \\
\hline Burn-leach (DRF-26B) $(\mu g)$ : & 52.91 & 46.51 & 55.06 & 52.70 & & \\
\hline Total leached $(\mu \mathrm{g}):$ & 82.61 & 80.36 & 82.10 & 81.90 & & \\
\hline V outside SiC ( $\mu \mathrm{g} /$ compact): & 16.52 & 16.07 & 16.42 & 16.38 & 16.35 & 0.19 \\
\hline \multicolumn{7}{|l|}{\begin{tabular}{|l} 
Titanium and Vanadium \\
\end{tabular}} \\
\hline $\mathrm{Ti}+\mathrm{V}$ outside SiC ( $\mu \mathrm{g} /$ compact): & 19.37 & 18.93 & 19.12 & 18.99 & 19.10 & 0.19 \\
\hline
\end{tabular}

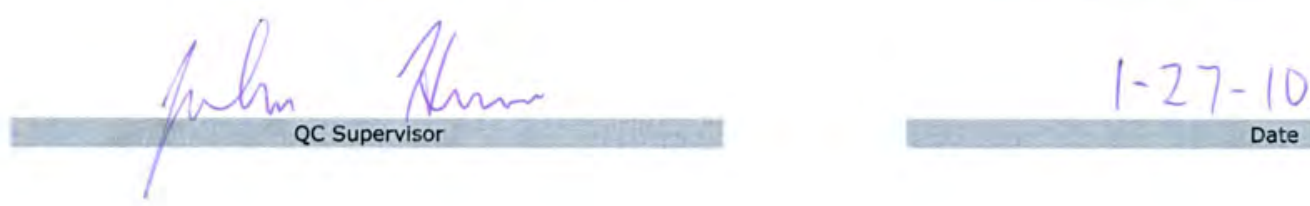


Inspection Report Form IRF-12B: Summary of Impurites Outside SiC - Maximum Corrected Values

\begin{tabular}{|r|l|}
\hline Procedure: & AGR-CHAR-PIP-12 Rev. 1 \\
\hline Operator: & Fred Montgomery \\
\hline Compact lot ID: & LEU09-OP2-Z \\
\hline Compact Lot description: & AGR-2 UCO Variant, from G73J-14-93073A \\
\hline
\end{tabular}

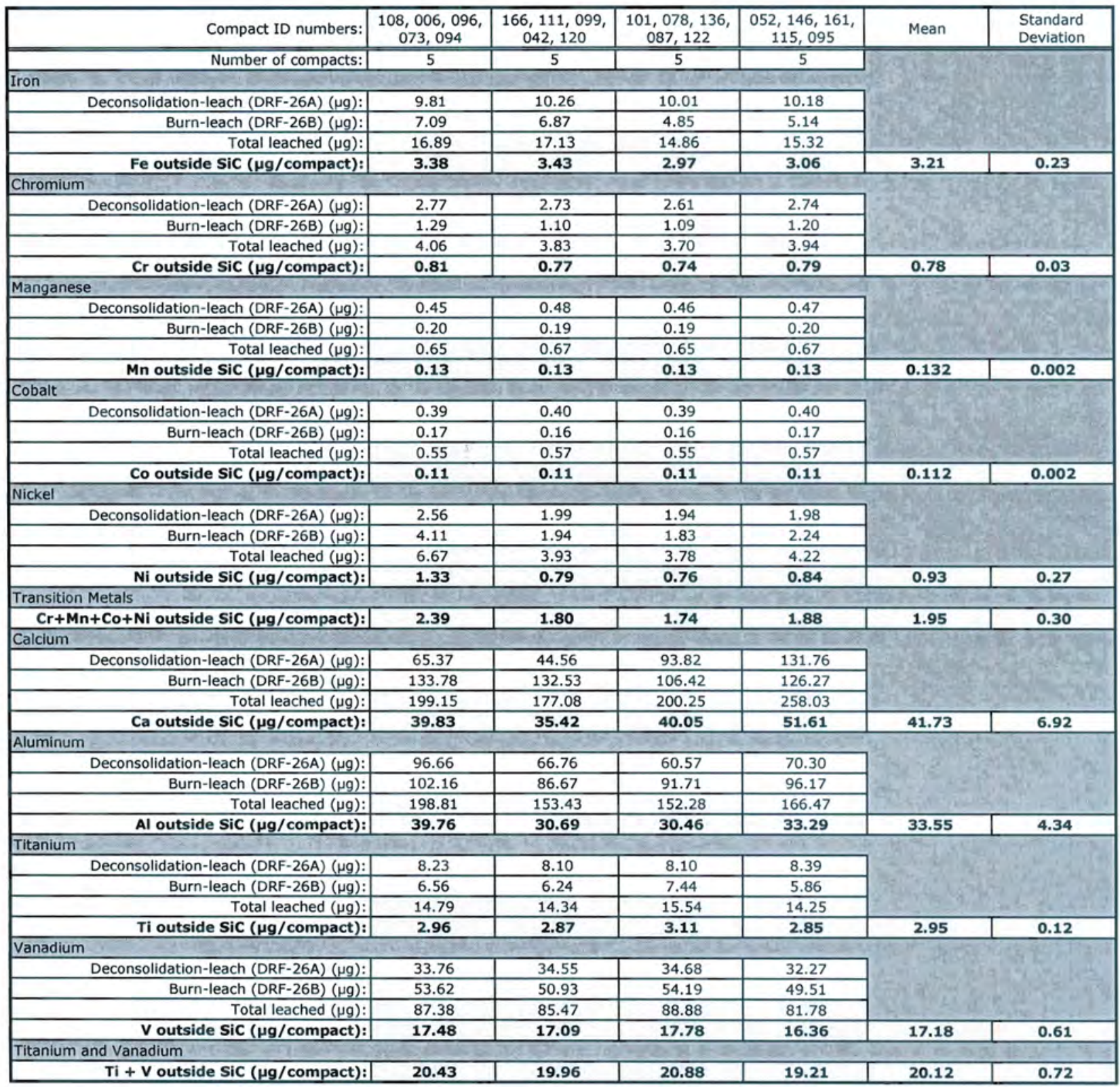

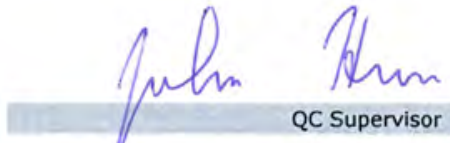

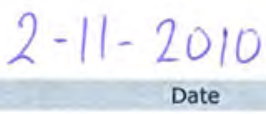


ORNL/TM-2010/017

Inspection Report Form IRF-12C: Summary of Uranium Contamination

Procedure: AGR-CHAR-PIP-12 Rev. 0

Operator: Fred Montgomery

Compact Lot description: AGR-2 UCO Variant, from G73J-14-93073A

\begin{tabular}{|r|c|c|c|c|c|}
\hline Compact ID numbers: & $\begin{array}{c}011,152,001, \\
068,046\end{array}$ & $\begin{array}{c}067,081,019, \\
008,032\end{array}$ & $\begin{array}{c}039,036,155, \\
012,117\end{array}$ & $\begin{array}{c}031,149,093, \\
147,072\end{array}$ & Total \\
\hline \hline Number of compacts: & 5 & 5 & 5 & 5 & 20 \\
\hline Effective number of exposed kernels: & 0.0 & 0.0 & 0.0 & 0.0 & 0.1 \\
\hline
\end{tabular}

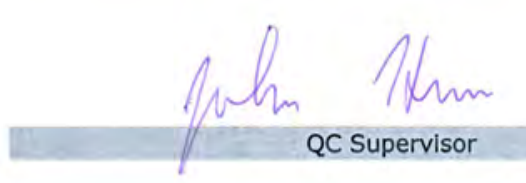

$$
1-27-10
$$

Date

51 
Inspection Report Form IRF-12C: Summary of Uranium Contamination

\begin{tabular}{|r|l|}
\hline Procedure: & AGR-CHAR-PIP-12 Rev. 0 \\
\hline Operator: & Fred Montgomery \\
\hline Compact lot ID: & LEU09-OP2-Z \\
\hline Compact Lot description: & AGR-2 UCO Variant, from G73J-14-93073A \\
\hline
\end{tabular}

\begin{tabular}{|r|c|c|c|c|c|}
\hline Compact ID numbers: & $\begin{array}{c}051,086,143, \\
097,033\end{array}$ & $\begin{array}{c}030,024,090, \\
013,010\end{array}$ & $\begin{array}{c}021,035,121, \\
107,061\end{array}$ & $\begin{array}{c}151,003,058, \\
080,009\end{array}$ & Total \\
\hline \hline Number of compacts: & 5 & 5 & 5 & 5 & 20 \\
\hline Effective number of exposed kernels: & 0.0 & 0.0 & 0.9 & 0.0 & 1.0 \\
\hline
\end{tabular}

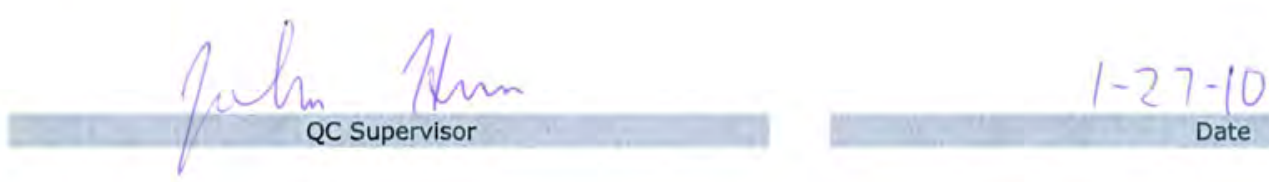


Inspection Report Form IRF-12C: Summary of Uranium Contamination

\begin{tabular}{|c|c|}
\hline Procedure: & AGR-CHAR-PIP-12 Rev. 0 \\
\hline Operator: & Fred Montgomery \\
\hline Compact lot ID: & LEU09-OP2-Z \\
\hline Compact Lot description: & AGR-2 UCO Variant, from G73J-14-93073A \\
\hline
\end{tabular}

\begin{tabular}{|r|c|c|c|c|c|}
\hline Compact ID numbers: & $\begin{array}{c}118,041,138, \\
007,071\end{array}$ & $\begin{array}{c}144,048,023, \\
037,047\end{array}$ & $\begin{array}{c}150,065,116,034,084,074, \\
140,160\end{array}$ & $\begin{array}{c}\text { Total } \\
159,060\end{array}$ \\
\hline \hline Number of compacts: & 5 & 5 & 5 & 5 & 20 \\
\hline Effective number of exposed kernels: & 0.0 & 0.0 & 0.8 & 0.0 & 0.9 \\
\hline
\end{tabular}

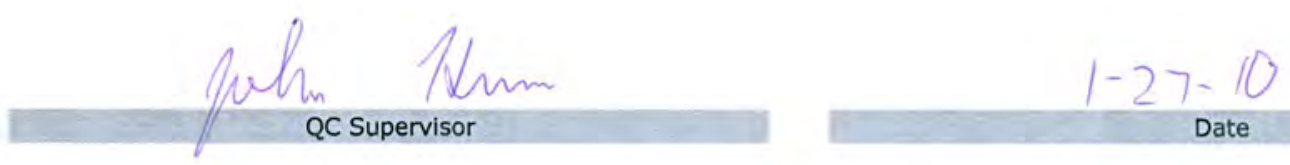




\section{Inspection Report Form IRF-12C: Summary of Uranium Contamination}

Procedure: AGR-CHAR-PIP-12 Rev. 0

Operator: Fred Montgomery

Compact lot ID: LEU09-OP2-Z

Compact Lot description: AGR-2 UCO Variant, from G73J-14-93073A

\begin{tabular}{|r|c|c|c|c|c|}
\hline Compact ID numbers: & $\begin{array}{c}050,044,020, \\
164,098\end{array}$ & $\begin{array}{c}027,157,054, \\
109,070\end{array}$ & $\begin{array}{c}025,106,133, \\
110,029\end{array}$ & $\begin{array}{c}113,162,055, \\
137,123\end{array}$ & Total \\
\hline \hline Number of compacts: & 5 & 5 & 5 & 5 & 20 \\
\hline Effective number of exposed kernels: & 0.0 & 0.9 & 0.0 & 0.0 & 1.0 \\
\hline
\end{tabular}

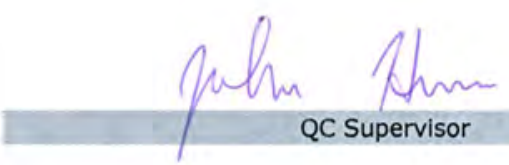

$1-27-10$ 
Inspection Report Form IRF-12C: Summary of Uranium Contamination

\begin{tabular}{|r|l|}
\hline Procedure: & AGR-CHAR-PIP-12 Rev. 0 \\
\hline Operator: & Fred Montgomery \\
\hline Compact lot ID: & LEU09-OP2-Z \\
\hline Compact Lot description: & AGR-2 UCO Variant, from G73J-14-93073A \\
\hline
\end{tabular}

\begin{tabular}{|r|c|c|c|c|c|}
\hline Compact ID numbers: & $\begin{array}{c}108,006,096, \\
073,094\end{array}$ & $\begin{array}{c}166,111,099, \\
042,120\end{array}$ & $\begin{array}{c}101,078,136, \\
087,122\end{array}$ & $\begin{array}{c}\text { 052, 146, 161, } \\
115,095\end{array}$ & Total \\
\hline \hline Number of compacts: & 5 & 5 & 5 & 5 & 20 \\
\hline Effective number of exposed kernels: & 0.0 & 0.0 & 0.0 & 0.0 & 0.1 \\
\hline
\end{tabular}

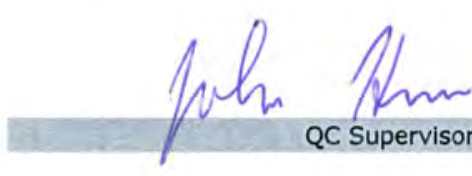

$$
2-11-2010
$$




\section{Inspection Report Form IRF-12D: Summary of Burn Leach Defects}

\begin{tabular}{|c|c|}
\hline Procedure: & AGR-CHAR-PIP-12 Rev. 0 \\
\hline Operator: & Fred Montgomery \\
\hline Compact lot ID: & LEU09-OP2-Z \\
\hline Compact Lot description: & AGR-2 UCO Variant, from G73J-14-93073 A \\
\hline
\end{tabular}

\begin{tabular}{|r|c|c|c|c|c|}
\hline Compact ID numbers: & $\begin{array}{c}011,152,001, \\
068,046\end{array}$ & $\begin{array}{c}067,081,019, \\
008,032\end{array}$ & $\begin{array}{c}039,036,155, \\
012,117\end{array}$ & $\begin{array}{c}031,149,093, \\
147,072\end{array}$ & Total \\
\hline \hline Number of compacts: & 5 & 5 & 5 & 5 & 20 \\
\hline Number of leached kernels: & 0 & 0 & 0 & 0 & 0 \\
\hline
\end{tabular}

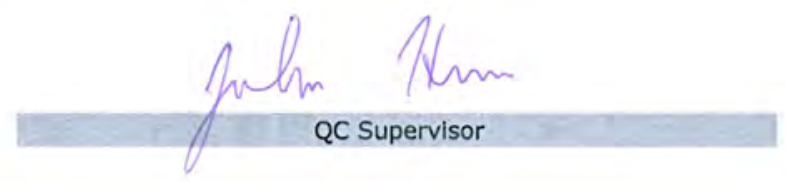

$$
\frac{1-27-10}{\text { Date }}
$$




\section{Inspection Report Form IRF-12D: Summary of Burn Leach Defects}

Procedure: AGR-CHAR-PIP-12 Rev. 0

Operator: Fred Montgomery

Compact lot ID: LEU09-OP2-Z

Compact Lot description: AGR-2 UCO Variant, from G73J-14-93073A

\begin{tabular}{|r|c|c|c|c|c|}
\hline Compact ID numbers: & $\begin{array}{c}051,086,143, \\
097,033\end{array}$ & $\begin{array}{c}030,024,090, \\
013,010\end{array}$ & $\begin{array}{c}021,035,121, \\
107,061\end{array}$ & $\begin{array}{c}151,003,058, \\
080,009\end{array}$ & Total \\
\hline \hline Number of compacts: & 5 & 5 & 5 & 5 & 20 \\
\hline Number of leached kernels: & 0 & 0 & 0 & 0 & 0 \\
\hline
\end{tabular}

Inin Mm

QC Supervisor

\section{$1-27-10$}

Date 


\section{Inspection Report Form IRF-12D: Summary of Burn Leach Defects}

Procedure: AGR-CHAR-PIP-12 Rev. 0

Operator: Fred Montgomery

Compact lot ID: LEU09-OP2-Z

Compact Lot description: AGR-2 UCO Variant, from G73J-14-93073A

\begin{tabular}{|r|c|c|c|c|c|}
\hline Compact ID numbers: & $\begin{array}{c}050,044,020, \\
164,098\end{array}$ & $\begin{array}{c}027,157,054, \\
109,070\end{array}$ & $\begin{array}{c}025,106,133, \\
110,029\end{array}$ & $\begin{array}{c}113,162,055, \\
137,123\end{array}$ & Total \\
\hline \hline Number of compacts: & 5 & 5 & 5 & 5 & 20 \\
\hline Number of leached kernels: & 0 & 0 & 0 & 0 & 0 \\
\hline
\end{tabular}

Mun Mim

QC Supervisor

$$
1-27-10
$$

Date 


\section{Inspection Report Form IRF-12D: Summary of Burn Leach Defects}

Procedure: AGR-CHAR-PIP-12 Rev. 0

Operator: Fred Montgomery

Compact lot ID: LEU09-OP2-Z

Compact Lot description: AGR-2 UCO Variant, from G73J-14-93073A

\begin{tabular}{|r|c|c|c|c|c|}
\hline Compact ID numbers: & $\begin{array}{c}108,006,096, \\
073,094\end{array}$ & $\begin{array}{c}166,111,099, \\
042,120\end{array}$ & $\begin{array}{c}101,078,136, \\
087,122\end{array}$ & $\begin{array}{c}052,146,161, \\
115,095\end{array}$ & Total \\
\hline \hline Number of compacts: & 5 & 5 & 5 & 5 & 20 \\
\hline Number of leached kernels: & 0 & 0 & 0 & 0 & 0 \\
\hline
\end{tabular}

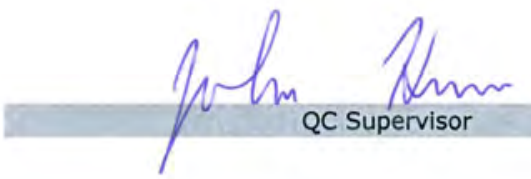

$$
2-11-2010
$$

Date 
ORNL/TM-2010/017
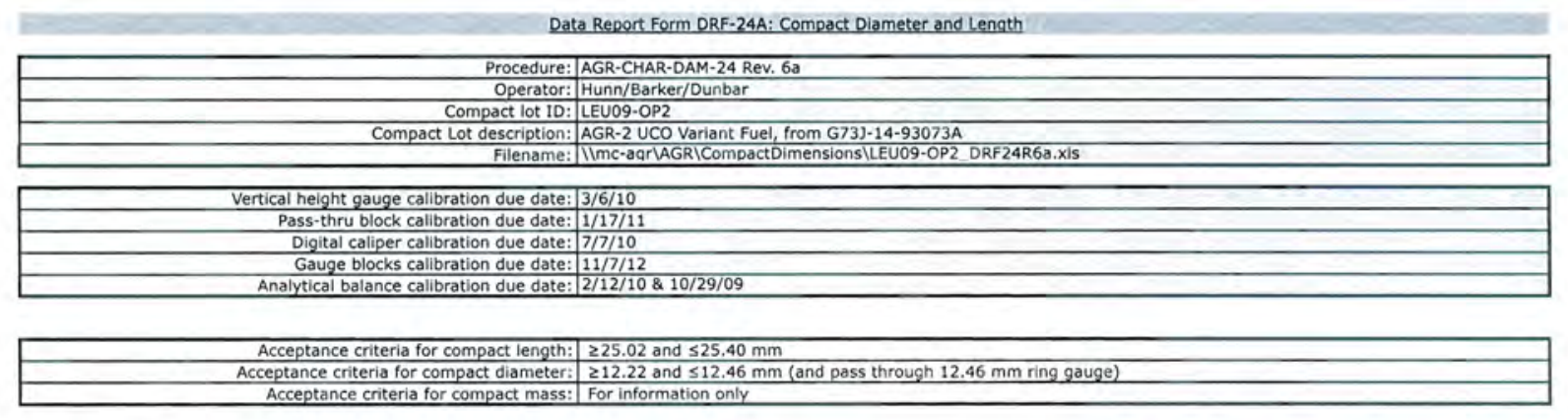

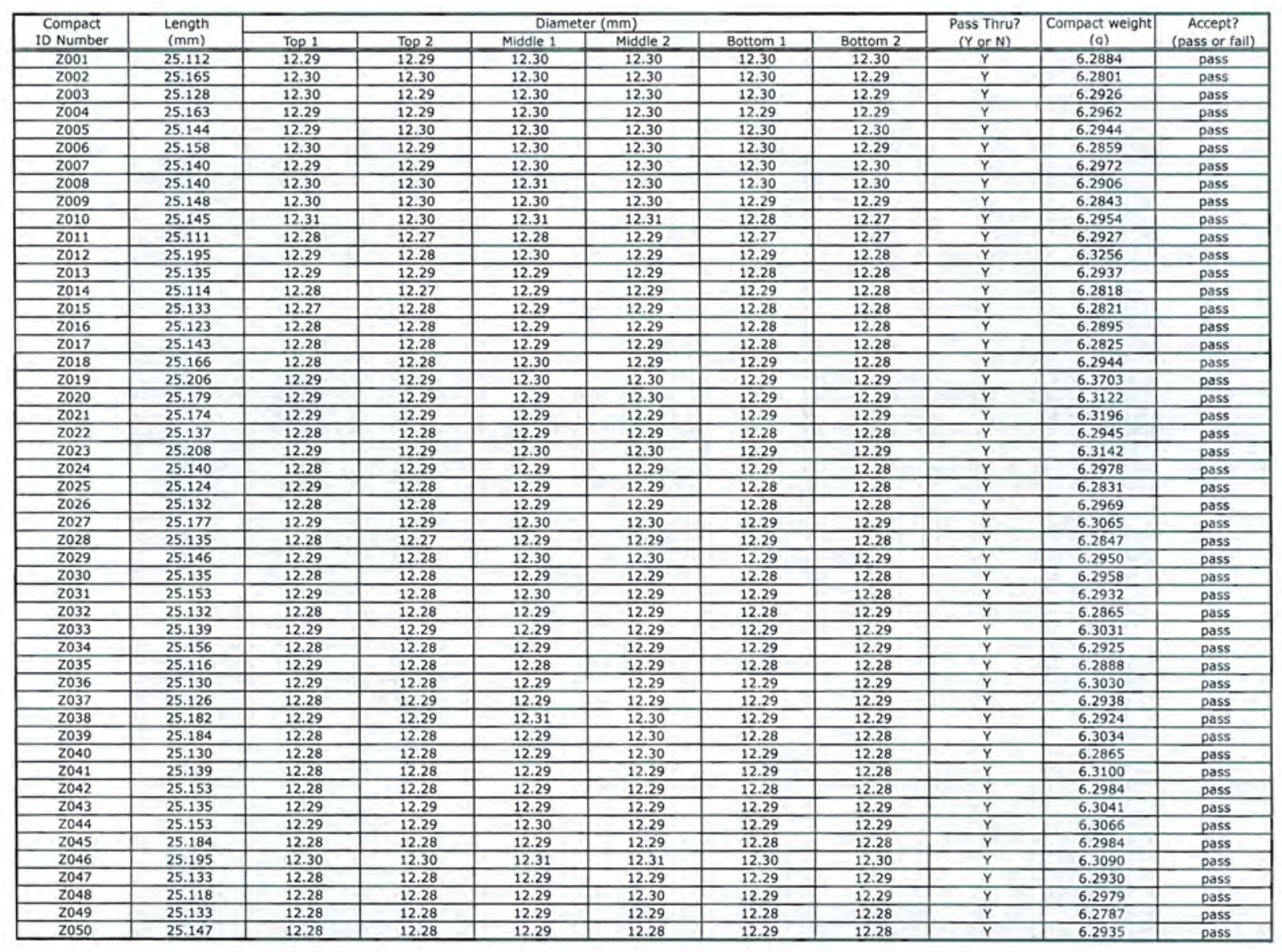

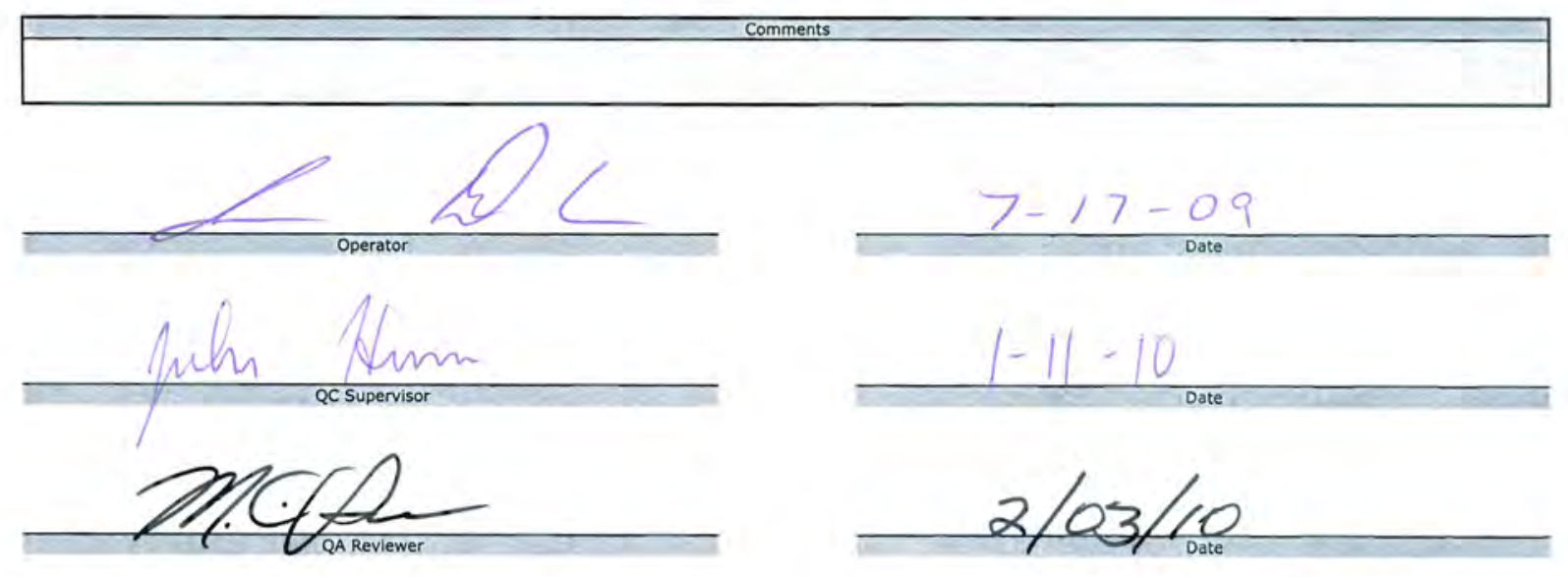

60 
ORNL/TM-2010/017

Data Report Form DRF-24A: Compact Diameter and Length

Procedure: AGR-CHAR-DAM-24 Rev. $6 a$

Operator: Hunn/Barker/Dunbar Compact lot ID: $L$ LEU 09-OP2

\begin{tabular}{r|r} 
Compact Lot description: & AGR -2 UCO Variant Fuel, from G73J-14-93073A \\
\hline Filename: & |lmc-aqrlAGRICompactDimensions ILEU09-OP2_DRF24R6a.xis
\end{tabular}

Vertical height gauge calibration due date: $3 / 6 / 10$

Pass-thru block calibration due date: $1 / 17 / 11$

Digital caliper calibration due date: $7 / 7 / 10$

Analytical balance calibration due date: $\frac{11 / 1 / 12}{2 / 10} \& 10 / 29 / 09$

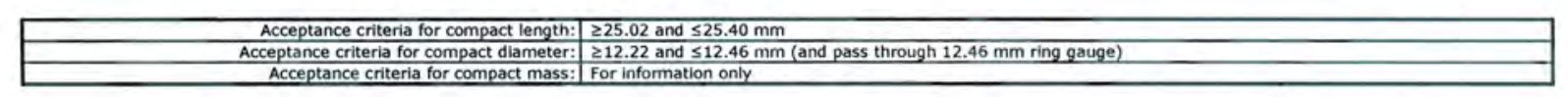

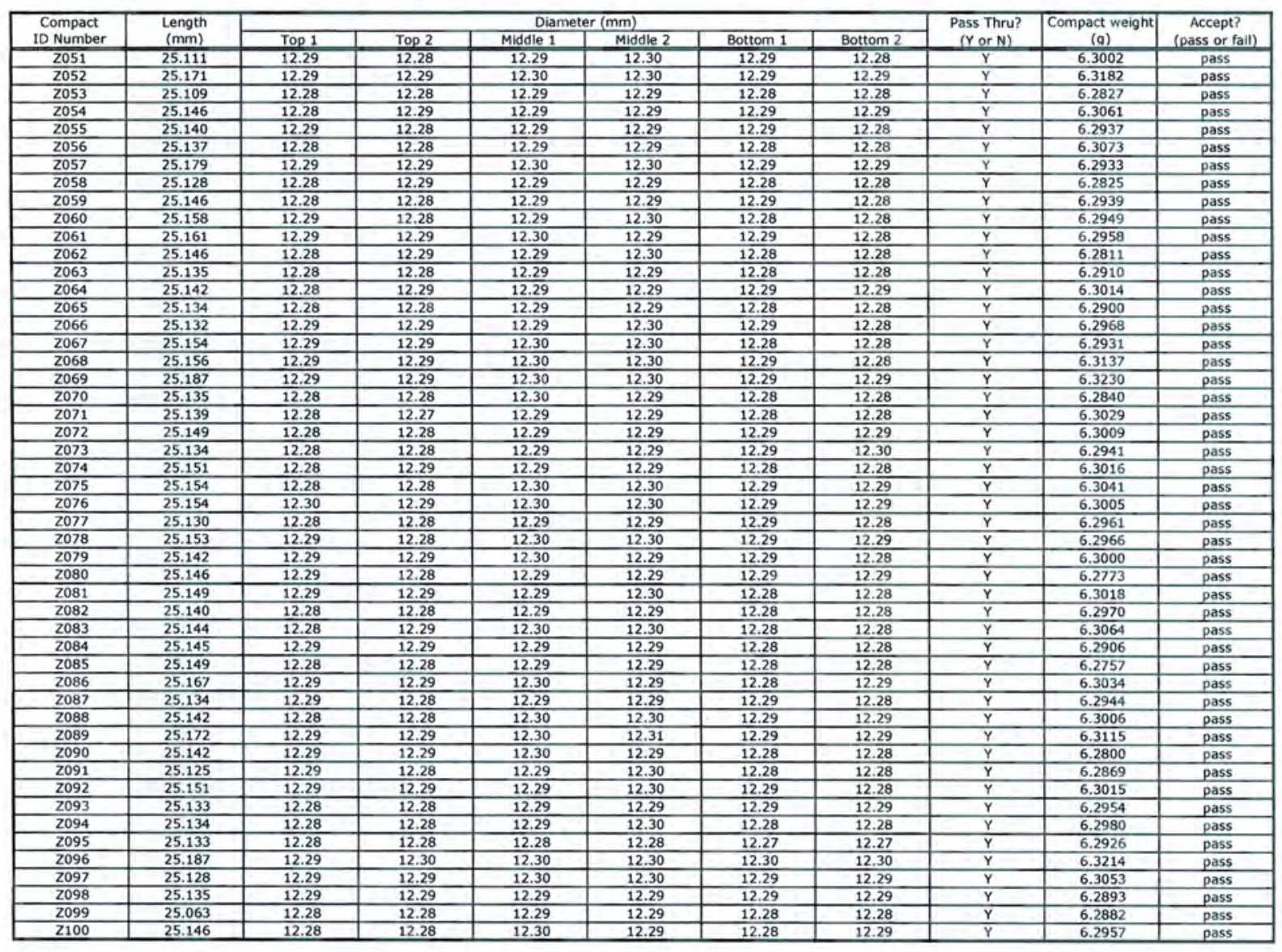
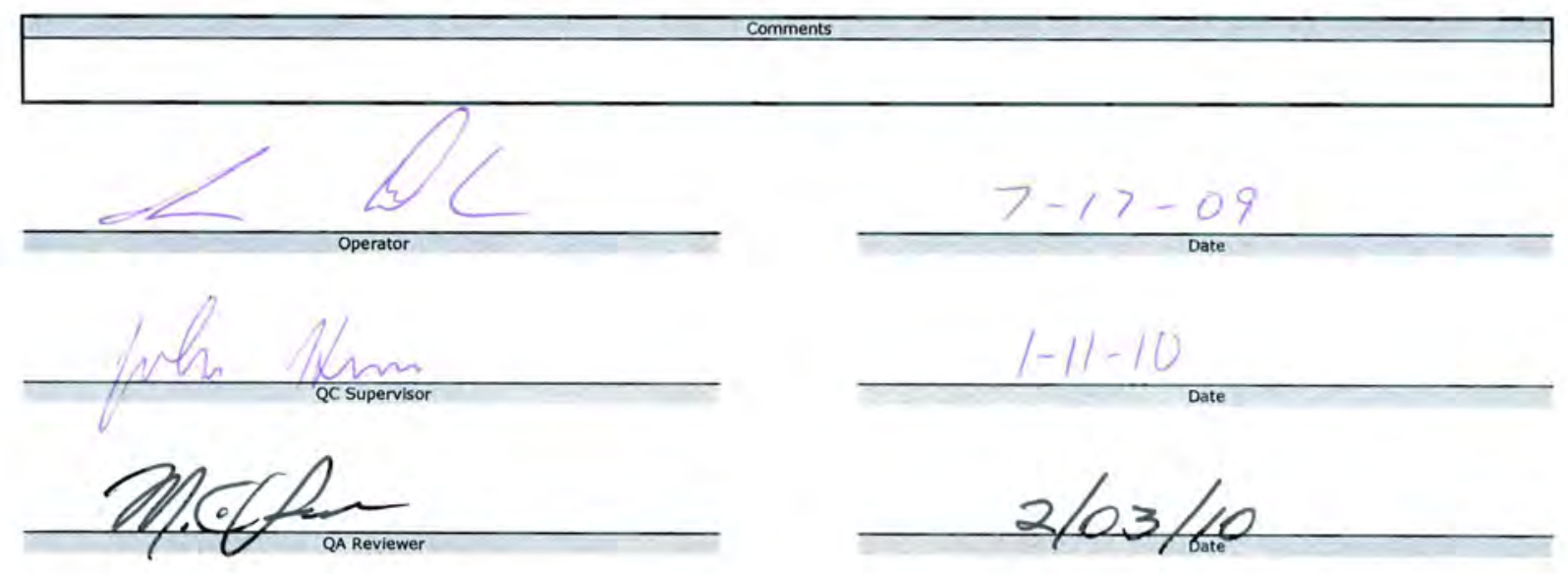

61 


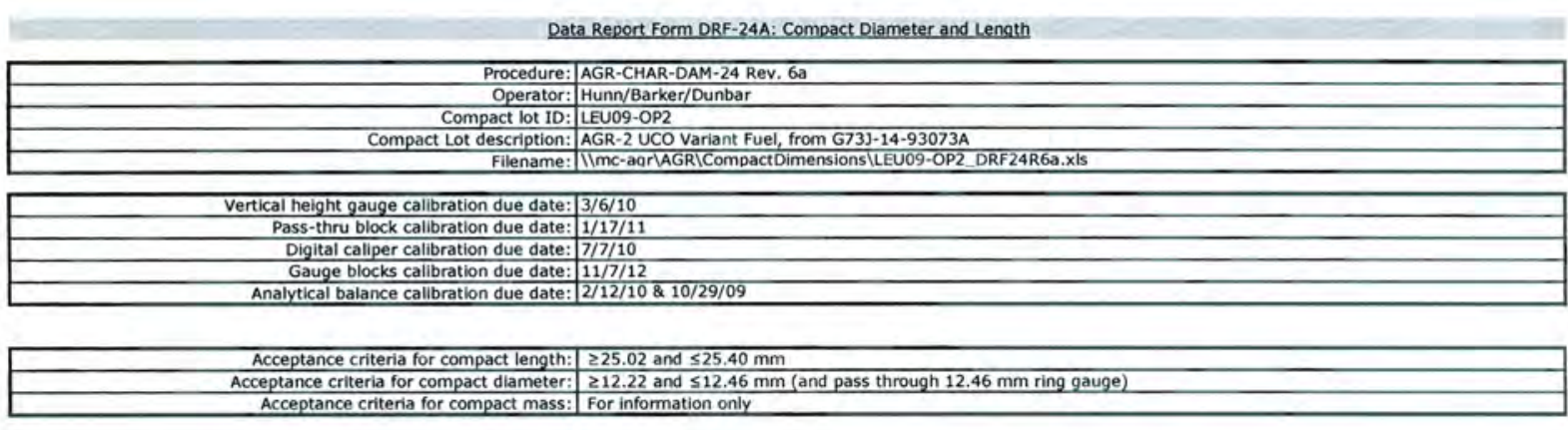

\begin{tabular}{|c|c|c|c|c|c|c|c|c|c|c|}
\hline Compact & Length & \multicolumn{6}{|c|}{ Diameter $(\mathrm{mm})$} & Pass Thru? & Compact weight & Accept? \\
\hline ID Number & $(\mathrm{mm})$ & Top 1 & Top 2 & Middle 1 & Middle 2 & Bottom 1 & Bottom 2 & $(\gamma$ or $N)$ & (a) & (pass or fail) \\
\hline 2101 & 25.129 & 12.29 & 12.29 & 12.30 & 12.30 & 12.29 & 12.28 & $\frac{Y}{Y}$ & 6.3040 & pass \\
\hline$\frac{Z 102}{Z 103}$ & $\frac{225.125}{25.149}$ & $\frac{12.28}{12.28}$ & $\frac{12.28}{12.29}$ & $\frac{12.30}{12.28}$ & $\frac{12.30}{12.28}$ & $\frac{12.29}{12.28}$ & $\frac{12.28}{12.28}$ & $\frac{y}{y}$ & $\frac{6.2944}{6.2859}$ & $\begin{array}{l}\text { pass } \\
\text { pass }\end{array}$ \\
\hline $\mathrm{Z104}$ & 25.153 & 12.28 & 12.28 & 12.29 & 12.29 & 12.28 & 12.28 & $\bar{Y}$ & $\frac{0.2059}{6.3137}$ & pass \\
\hline$Z 105$ & 25.115 & 12.29 & 12.29 & 12.30 & 12.30 & 12.28 & 12.28 & $Y$ & 6.2989 & pass \\
\hline Z106 & 25.120 & 12.28 & 12.28 & 12.29 & 12.29 & 12.28 & 12.28 & $Y$ & 6.2897 & pass \\
\hline Z107 & 25.151 & 12.29 & 12.29 & 12.29 & 12.29 & 12.29 & 12.28 & Y & 6.2884 & pass \\
\hline Z108 & 25.024 & 12.28 & 12.27 & 12.28 & 12.29 & 12.27 & 12.28 & $\gamma$ & 6.2840 & pass \\
\hline Z109 & 25.152 & 12.29 & 12.29 & 12.29 & 12.29 & 12.28 & 12.28 & Y & 6.2941 & pass \\
\hline Z110 & 25.151 & 12.28 & 12.28 & 12.29 & 12.29 & 12.28 & 12.28 & y & 6.2837 & pass \\
\hline Z1111 & 25.152 & 12.29 & 12.28 & 12.29 & 12.29 & 12.28 & 12.28 & y & 6.2904 & pass \\
\hline 2112 & 25.134 & 12.29 & 12.29 & 12.29 & 12.29 & 12.28 & 12.28 & r & 6.2922 & pass \\
\hline $\mathrm{ZZ113}$ & 25.114 & 12.28 & 12.28 & 12.29 & 12.29 & 12.28 & 12.29 & Y & 6.2861 & pass \\
\hline 2114 & 25.130 & 12.28 & 12.28 & 12.29 & 12.29 & 12.29 & 12.29 & Y & 6.2934 & pass \\
\hline 2115 & 25.132 & 12.28 & 12.28 & 12.29 & 12.29 & 12.28 & 12.28 & Y & 6.2832 & pass \\
\hline 2116 & 25.142 & 12.29 & 12.28 & 12.29 & 12.29 & 12.28 & 12.29 & $\begin{array}{r}Y \\
\end{array}$ & 6.2870 & pass \\
\hline Z117 & 25.148 & 12.29 & 12.29 & 12.29 & 12.29 & 12.28 & 12.29 & Y & 6.3025 & pass \\
\hline 2118 & 25.151 & 12.28 & 12.28 & 12.29 & 12.30 & 12.28 & 12.28 & $Y$ & 6.2928 & pass \\
\hline 2119 & 25.158 & 12.28 & 12.28 & 12.30 & 12.30 & 12.29 & 12.29 & $Y$ & 6.2985 & pass \\
\hline 2120 & 25.120 & 12.29 & 12.29 & 12.30 & 12.30 & 12.28 & 12.29 & Y & 6.3053 & pass \\
\hline $\mathrm{Z121}$ & 25.135 & 12.29 & $\frac{12.28}{128}$ & 12.29 & 12.29 & 12.29 & 12.28 & $\begin{array}{r}Y \\
Y\end{array}$ & 6.2959 & pass \\
\hline$\frac{12122}{7122}$ & 25.130 & 12.28 & 12.28 & 12.30 & 12.29 & 12.28 & 12.28 & $Y$ & 6.2843 & pass \\
\hline$\frac{2123}{8124}$ & 25.151 & 12.28 & 12.28 & 12.29 & 12.29 & 12.29 & 12.29 & $y$ & 6.2986 & pass \\
\hline$\frac{124}{8725}$ & $\frac{23.130}{25.130}$ & 12.29 & 12.29 & 12.29 & 12.29 & 12,28 & 12.28 & $\frac{y}{y}$ & 6.2830 & pass \\
\hline$\frac{1123}{7125}$ & $\frac{25.139}{251.39}$ & 12.49 & 12.28 & 12.29 & 12.30 & 12.28 & 12.29 & $\frac{r}{y}$ & 6.3026 & pass \\
\hline$\frac{1420}{7127}$ & $\frac{25.134}{25.12}$ & 12.28 & 12.27 & $\frac{12.30}{1.32}$ & $\frac{12.30}{12.30}$ & 12.28 & $\frac{12.29}{1.29}$ & $\frac{1}{4}$ & 6.2944 & pass \\
\hline 2128 & $\frac{23.142}{550}$ & $\frac{12.28}{1228}$ & $\frac{12.280}{1028}$ & 12.29 & 12.50 & 10.20 & $\frac{12.28}{1.37}$ & $\frac{1}{x}$ & 0.2080 & pass \\
\hline$\frac{1280}{7129}$ & 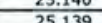 & $\frac{12,20}{1228}$ & $\frac{12.280}{1028}$ & $\frac{12.29}{103}$ & 12.29 & 12.28 & 12.29 & $\frac{1}{x}$ & 0.2880 & pass \\
\hline$\frac{129}{7130}$ & $\frac{25.353}{25.132}$ & 12.28 & 12.60 & 12.30 & $\frac{1.30}{2.30}$ & $\frac{12.29}{103}$ & $\frac{12.29}{1.3}$ & $\frac{y}{y}$ & 0.2837 & pass \\
\hline $\mathrm{Z} 131$ & 25.142 & 12.28 & 1228 & 1220 & 1279 & 1228 & 1278 & $\bar{y}$ & 0.23050 & pass \\
\hline $\mathrm{Z132}$ & 25.217 & 12.28 & 12.28 & 12.30 & 12.29 & 12.27 & 12.28 & $\frac{1}{y}$ & 6.3422 & pass \\
\hline Z133 & 25.133 & 12.29 & 12.28 & 12.29 & 12.29 & 12.29 & 12.28 & $\bar{Y}$ & 6.2959 & pass \\
\hline Z134 & 25.147 & 12.29 & 12.29 & 12.30 & 12.30 & 12.29 & 12.28 & $y$ & 6.2983 & pass \\
\hline Z135 & 25.133 & 12.28 & 12.28 & 12.29 & 12.29 & 12.28 & 12.28 & $Y$ & 6.2972 & pass \\
\hline Z136 & 25,140 & 12.29 & 12.28 & 12.29 & 12.29 & 12.28 & 12.29 & Y & 6.2922 & pass \\
\hline Z137 & 25.144 & 12.29 & 12.28 & 12.29 & 12.30 & 12.28 & 12.28 & $\gamma$ & 6.2877 & pass \\
\hline Z138 & 25.177 & 12.29 & 12.29 & 12.30 & 12.30 & 12.29 & 12.28 & Y & 6.3043 & pass \\
\hline 2139 & 25.112 & 12.28 & 12.27 & 12.29 & 12.29 & 12.28 & 12.28 & $\gamma$ & 6.2862 & pass \\
\hline $\mathrm{Z} 1140$ & 25.161 & 12.28 & 12.28 & 12.29 & 12.29 & 12.29 & 12.29 & $\gamma$ & 6.2964 & pass \\
\hline $\mathrm{Z} 141$ & 25.132 & 12.28 & 12.28 & 12.29 & 12.29 & 12.28 & 12.28 & Y & 6.2819 & pass \\
\hline 2142 & 25.114 & 12.28 & 12.28 & 12.29 & 12.30 & 12.28 & 12.28 & Y & 6.2926 & pass \\
\hline 2143 & 25.148 & 12.29 & 12.29 & 12.29 & 12.28 & 12.28 & 12.28 & Y & 6.2894 & pass \\
\hline 2144 & 25.156 & 12.28 & 12.28 & 12.29 & 12.29 & 12.28 & 12.27 & Y & 6.2958 & pass \\
\hline $\mathrm{Z} 145$ & 25.134 & 12.28 & 12.29 & 12.29 & 12.29 & 12.28 & 12.28 & Y & 6.2905 & pass \\
\hline 2146 & 25.163 & 12.29 & 12.28 & 12.30 & 12.29 & 12.28 & 12.29 & y & 6.3008 & pass \\
\hline 2147 & 25.158 & 12.29 & 12.29 & 12.30 & 12.30 & 12.28 & 12.28 & Y & 6.3024 & pass \\
\hline 2148 & 20.142 & 12.28 & 12.28 & 12.29 & 12.30 & 12.28 & 12.29 & $\begin{array}{r}Y \\
Y\end{array}$ & 6.2992 & pass \\
\hline$\frac{2149}{7150}$ & $\frac{25.115}{25.148}$ & $\frac{12.28}{12.20}-x-y$ & 12.28 & $\frac{12.29}{12.70}$ & $\frac{12.30}{12.30}$ & 12.28 & 12.28 & - & 6.2842 & pass \\
\hline Z150 & 25.148 & 12.29 & 12.28 & 12.30 & 12.30 & 12.29 & 12.28 & Y & 6.2950 & pass \\
\hline
\end{tabular}
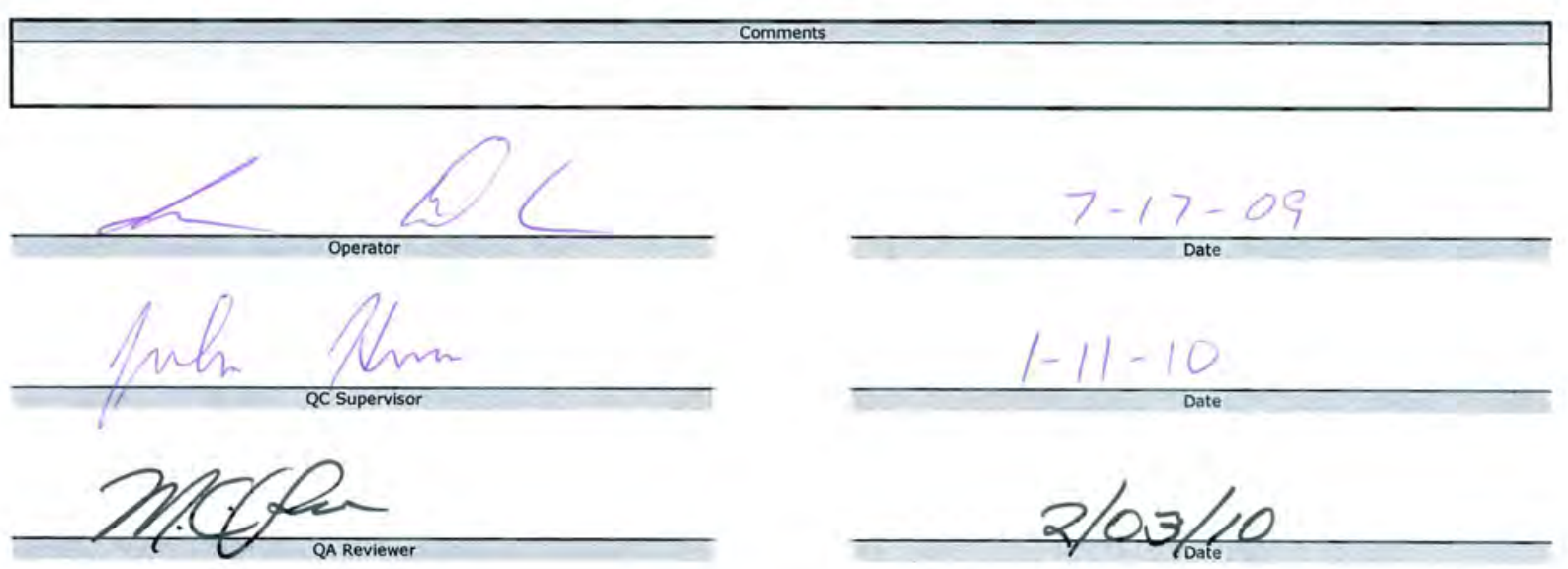
ORNL/TM-2010/017

Data Report Form DRF-24A: Compact Diameter and Length

Procedure: AGR-CHAR-DAM-24 Rev, Ga

Operator: Hunn/Barker/Dunbar

\begin{tabular}{r|l} 
Compact lot ID: & LEU 09-OP2 \\
\hline Compact Lot description: & AGR-2 UCO Variant Fuel, from G73J-14-93073A \\
\hline Filename: & (IImc-aqrVAGRICompactDimensions ILEU09-OP2 DRF24R6a.XIS
\end{tabular}

$\begin{aligned} \text { Compact Lot description: } & \text { AGR-2 UCO Variant Fuel, from G73J-14-93073A } \\ \text { Filename: } & \text { IImc-aqr\AGRICompactDimensions \LE U09-OP2 DRF24R6a.xis }\end{aligned}$

Vertical height gauge calibration due date: $3 / 6 / 10$

Pass-thru block calibration due date: $1 / 17 / 11$

\begin{tabular}{ll} 
Digital caliper calibration due date: & $7 / 7 / 10$ \\
Gauge blocks calibration due date: & $11 / 7 / 12$ \\
\hline
\end{tabular}

Analytical balance calibration due date: $2 / 12 / 10 \& 10 / 29 / 09$

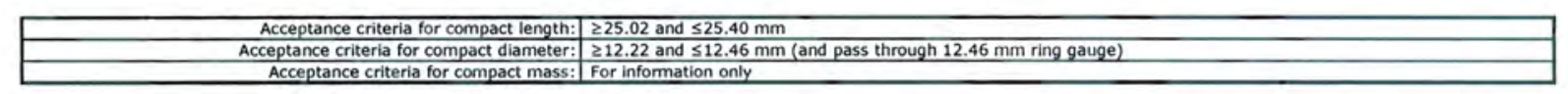

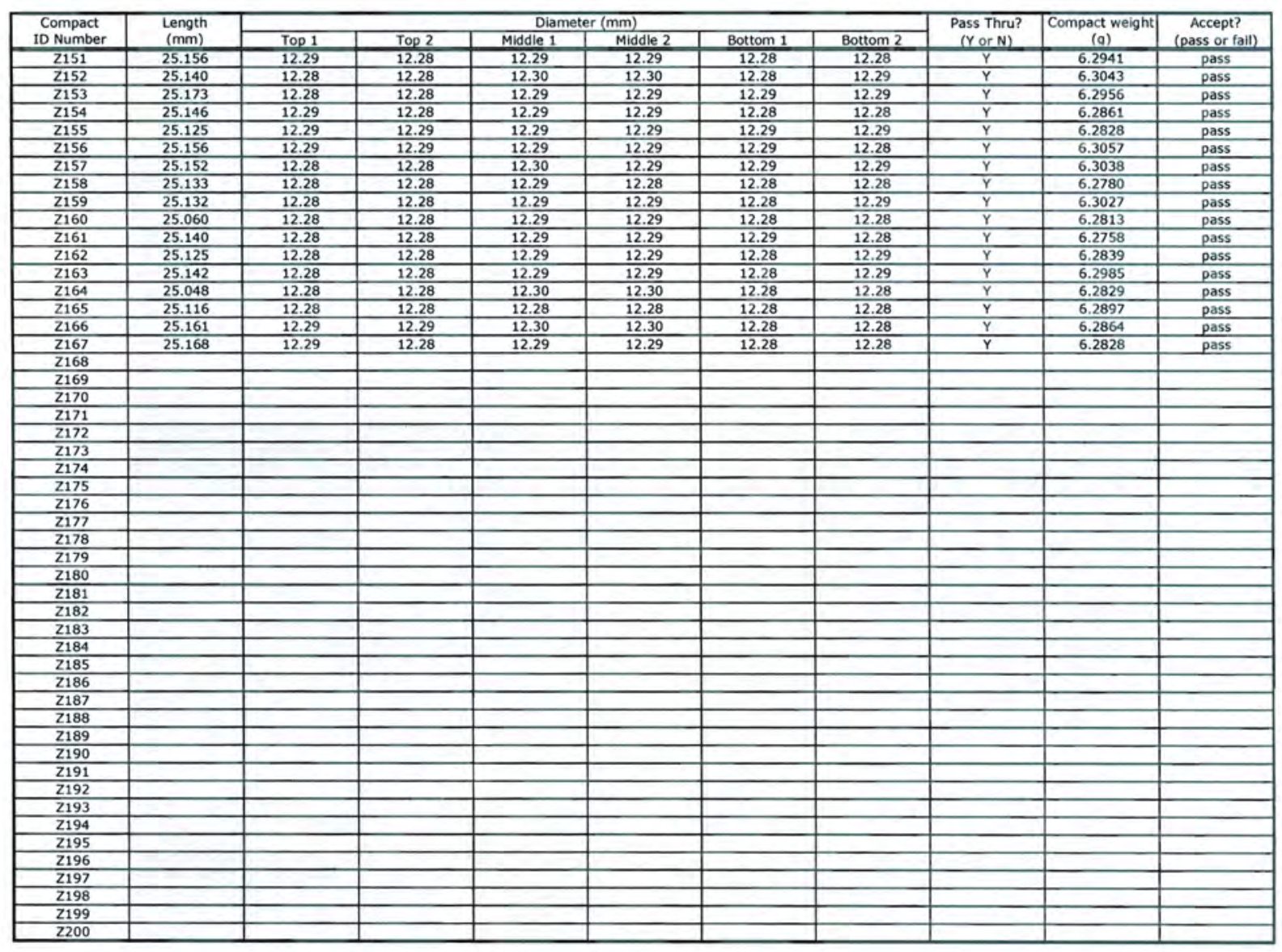
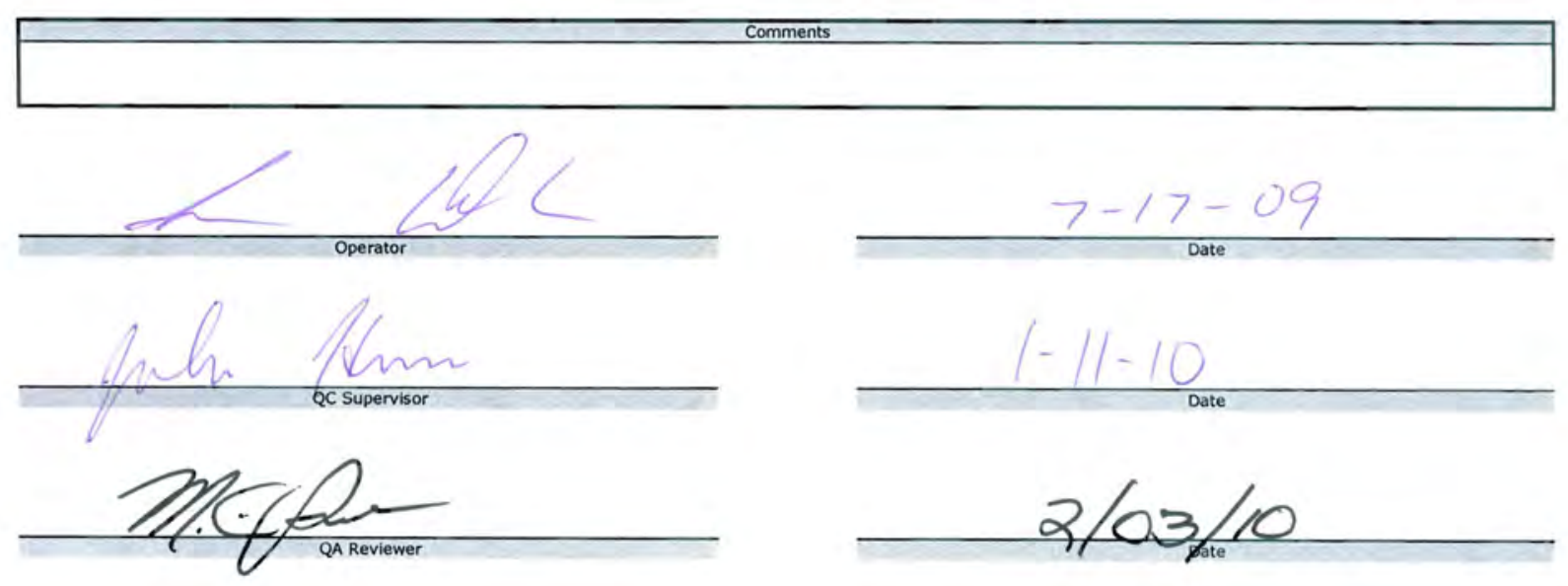

63 
Data Report Form DRF-24B: Compact Matrix Density

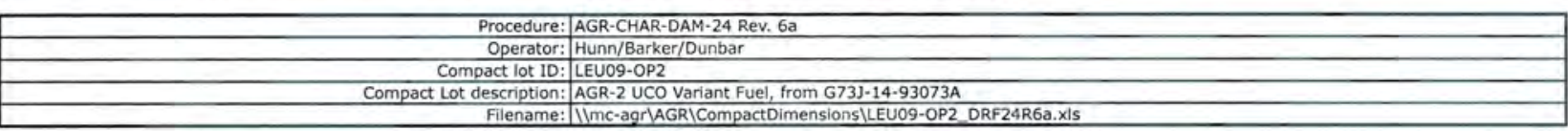

\begin{tabular}{|c|} 
Average weight per TRISO particle $(g): 1.032 \mathrm{E}-03$ \\
\hline Average weight per overcoated particle $(\mathrm{g}): 2.010 \mathrm{E}-03$ \\
\hline Average TRISO particle volume $(\mathrm{cm} 3): 3.430 \mathrm{E}-04$ \\
\hline
\end{tabular}

\section{Acceptance criteria for matrix density: $\geq 1.45$}

\begin{tabular}{|c|c|c|c|c|c|c|c|c|c|c|}
\hline $\begin{array}{c}\text { Compact } \\
\text { ID Number }\end{array}$ & $\begin{array}{l}\text { Compact Weight } \\
\text { (a) }\end{array}$ & $\begin{array}{l}\text { Length } \\
(\mathrm{mm})\end{array}$ & $\begin{array}{c}\text { Av. Diameter } \\
(\mathrm{mm})\end{array}$ & \begin{tabular}{|c|} 
Compact Volume \\
$(\mathrm{cm} 3)$
\end{tabular} & $\begin{array}{l}\text { Charge Weight } \\
\text { (a) }\end{array}$ & $\begin{array}{l}\text { Particle Weight } \\
\text { (a) }\end{array}$ & $\begin{array}{c}\text { Particle Volume } \\
(\mathrm{cm} 3)\end{array}$ & Packing Fraction & $\begin{array}{c}\text { Matrix Density } \\
(\mathrm{a} / \mathrm{cm} 3)\end{array}$ & $\begin{array}{c}\text { Accept? } \\
\text { (pass or fail) }\end{array}$ \\
\hline 2001 & 6.2884 & 25.112 & 12.30 & 2.98 & 6.4327 & 3.3024 & 1.10 & $37 \%$ & 1.58 & pass \\
\hline 2002 & 6.2801 & 25.165 & 12.30 & 2.99 & 6.4306 & 3.3014 & 1.10 & $37 \%$ & 1.57 & pass \\
\hline 2003 & 6.2926 & 25.128 & 12.30 & 2.98 & 6.4303 & 3.3014 & 1.10 & $37 \%$ & 1.59 & pass \\
\hline 2004 & 6.2962 & 25.163 & 12.29 & 2.99 & 6.4318 & 3.3024 & 1.10 & $37 \%$ & 1.58 & pass \\
\hline 2005 & 6.2944 & 25.144 & 12.30 & 2.99 & 6.4309 & 3.3014 & 1.10 & $37 \%$ & 1.58 & pass \\
\hline 2006 & 6.2859 & 25.158 & 12.30 & 2.99 & 6.4313 & 3.3024 & 1.10 & $37 \%$ & 1.58 & pass \\
\hline 2007 & 6.2972 & 25.140 & 12.30 & 2.99 & 6.4311 & 3.3024 & 1.10 & $37 \%$ & 1.59 & pass \\
\hline 2008 & 6.2906 & 25.140 & 12.30 & 2.99 & 6.4318 & 3.3024 & 1.10 & $37 \%$ & 1.58 & pass \\
\hline 2009 & 6.2843 & 25.148 & 12.30 & 2.99 & 6.4326 & 3.3024 & 1.10 & $37 \%$ & 1.58 & pass \\
\hline 2010 & 6.2954 & 25.145 & 12.30 & 2.99 & 6.4322 & 3.3024 & 1.10 & $37 \%$ & 1.58 & pass \\
\hline 2011 & 6.2927 & 25.111 & 12.28 & 2.97 & 6.4307 & 3.3014 & 1.10 & $37 \%$ & 1.60 & pass \\
\hline 2012 & 6.3256 & 25.195 & 12.29 & 2.99 & 6.4329 & 3.3024 & 1.10 & $37 \%$ & 1.60 & pass \\
\hline 2013 & 6.2937 & 25.135 & 12.29 & 2.98 & 6.4313 & 3.3024 & 1.10 & $37 \%$ & 1.59 & pass \\
\hline 2014 & 6.2818 & 25.114 & 12.28 & 2.98 & 6.4311 & 3.3024 & 1.10 & $37 \%$ & 1.59 & pass \\
\hline 2015 & 6.2821 & 25.133 & 12.28 & 2.98 & 6.4325 & 3.3024 & 1.10 & $37 \%$ & 1.59 & pass \\
\hline 2016 & 6.2895 & 25.123 & 12.28 & 2.98 & 6.4315 & 3.3024 & 1.10 & $37 \%$ & 1.59 & pass \\
\hline 2017 & 6.2825 & 25.143 & 12.28 & 2.98 & 6.4322 & 3.3024 & 1.10 & $37 \%$ & 1.58 & pass \\
\hline 2018 & 6.2944 & 25.166 & 12.29 & 2.98 & 6.4329 & 3.3024 & 1.10 & $37 \%$ & 1.59 & pass \\
\hline 2019 & 6.3703 & 25.206 & 12.29 & 2.99 & 6.4325 & 3.3024 & 1.10 & $37 \%$ & 1.62 & pass \\
\hline 2020 & 6.3122 & 25.179 & 12.29 & 2.99 & 6.4312 & 3.3024 & 1.10 & $37 \%$ & 1.59 & pass \\
\hline 2021 & 6.3196 & 25.174 & 12.29 & 2.99 & 6.4323 & 3.3024 & 1.10 & $37 \%$ & 1.60 & pass \\
\hline 2022 & 6.2945 & 25.137 & 12.28 & 2.98 & 6.4311 & 3.3024 & 1,10 & $37 \%$ & 1.59 & pass \\
\hline 2023 & 6.3142 & 25.208 & 12.29 & 2.99 & 6.4314 & 3.3024 & 1.10 & $37 \%$ & 1.59 & $\frac{p \text { pass }}{\text { pass }}$ \\
\hline 2024 & 6.2978 & 25.140 & 12.29 & 2.98 & 6.4302 & 3.3014 & 1.10 & $37 \%$ & 1.59 & pass \\
\hline 2025 & 6.2831 & 25.124 & 12.29 & 2.98 & 6.4302 & 3.3014 & 1.10 & $37 \%$ & 1.59 & pass \\
\hline 2026 & 6.2969 & 25.132 & 12.28 & 2.98 & 6.4314 & 3.3024 & 1.10 & $37 \%$ & 1.59 & pass \\
\hline 2027 & 6.3065 & 25.177 & 12.29 & 2.99 & 6.4320 & 3.3024 & 1.10 & $37 \%$ & 1.59 & pass \\
\hline 2028 & 6.2847 & 25.135 & 12.28 & 2.98 & 6.4320 & 3.3024 & 1.10 & $37 \%$ & 1.59 & pass \\
\hline 2029 & 6.2950 & 25.146 & 12.29 & 2.98 & 6.4328 & 3.3024 & 1.10 & $37 \%$ & 1.59 & pass \\
\hline 2030 & 6.2958 & 25.135 & 12.28 & 2.98 & 6.4304 & 3.3014 & 1.10 & $37 \%$ & 1.59 & pass \\
\hline 2031 & 6.2932 & 25.153 & 12.29 & 2.98 & 6.4321 & 3.3024 & 1.10 & $37 \%$ & 1.59 & pass \\
\hline 2032 & 6.2865 & 25.132 & 12.29 & 2.98 & 6.4308 & 3.3014 & 1.10 & $37 \%$ & 1.59 & pass \\
\hline 2033 & 6.3031 & 25.139 & 12.29 & 2.98 & 6.4326 & 3.3024 & 1.10 & $37 \%$ & 1.59 & pass \\
\hline 2034 & 6.2925 & 25.156 & 12.29 & 2.98 & 6.4325 & 3.3024 & 1.10 & $37 \%$ & 1.59 & pass \\
\hline 2035 & 6.2888 & 25.116 & 12.28 & 2.98 & 6.4310 & 3.3024 & 1.10 & $37 \%$ & 1.59 & pass \\
\hline 2036 & 6.3030 & 25.130 & 12.29 & 2.98 & 6.4326 & 3.3024 & 1.10 & $37 \%$ & 1.59 & pass \\
\hline 2037 & 6.2938 & 25.126 & 12.29 & 2.98 & 6.4309 & 3.3014 & 1.10 & $37 \%$ & 1.59 & pass \\
\hline 2038 & 6.2924 & 25.182 & 12,30 & 2.99 & 6.4326 & 3.3024 & 1.10 & $37 \%$ & 1.58 & $\begin{array}{l}\text { pass } \\
\text { pass }\end{array}$ \\
\hline 2039 & 6.3034 & 25.184 & 12.29 & 2.99 & 6.4310 & 3.3024 & 1.10 & $37 \%$ & 1.59 & pass \\
\hline 2040 & 6.2865 & 25.130 & 12.29 & 2.98 & 6.4327 & 3.3024 & 1.10 & $37 \%$ & 1.59 & pass \\
\hline 2041 & $\frac{6.2605}{6.3100}$ & $\frac{5.35}{25.139}$ & $\frac{12.29}{12.29}$ & $\frac{2.90}{2.98}$ & 6.43304 & $\begin{array}{l}3.3524 \\
3.3014\end{array}$ & 1.10 & $37 \%$ & 1.60 & $\frac{\text { pass }}{\text { pass }}$ \\
\hline$\frac{0041}{Z}$ & $\frac{6.3100}{6.2984}$ & $\frac{25.159}{25.153}$ & $\frac{12.29}{12.28}$ & $\frac{2.98}{2.98}$ & 6.40649 & 3.3014 & $\frac{1.10}{1.10}$ & $37 \%$ & $\begin{array}{l}1.60 \\
1.59\end{array}$ & $\begin{array}{l}\text { pass } \\
\text { pass }\end{array}$ \\
\hline 2043 & 6.3041 & 25.135 & 12.29 & 2.98 & 6.4320 & 3.3024 & 1.10 & $37 \%$ & 1.59 & pass \\
\hline 2044 & 6.3066 & 25.153 & 12.29 & 2.98 & 6.4309 & 3.3014 & 1.10 & $37 \%$ & 1.59 & pass \\
\hline Z045 & 6.2984 & 25.184 & 12.28 & 2.98 & 6.4311 & 3.3024 & 1.10 & $37 \%$ & 1.59 & pass \\
\hline Z046 & 6.3090 & 25.195 & 12,30 & 3.00 & 6.4311 & 3.3024 & 1.10 & $37 \%$ & 1.58 & pass \\
\hline 2047 & 6.2930 & 25.133 & 12.29 & 2.98 & 6.4316 & 3.3024 & 1.10 & $37 \%$ & 1.59 & pass \\
\hline 2048 & 6.2979 & 25.118 & 12.29 & 2.98 & 6.4306 & 3.3014 & 1.10 & & & pass \\
\hline 2049 & 6.2787 & 25.133 & 12.28 & $\frac{2.90}{2.98}$ & 6.4333 & 3.3034 & 1.10 & $37 \%$ & 1.58 & pass \\
\hline 2050 & 6.2935 & 25.147 & 12.28 & 2.98 & 6.4318 & 3.3024 & 1.10 & $37 \%$ & 1.59 & pass \\
\hline
\end{tabular}

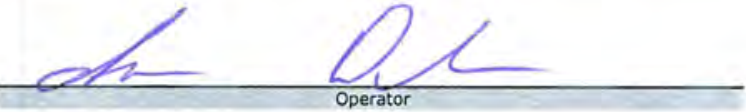

ling $1 / 2$

QC Supervisor

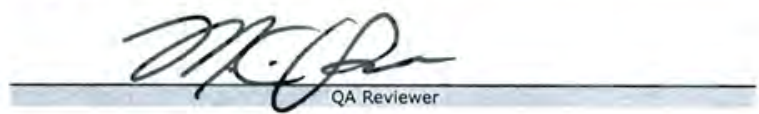

$7-17-09$

$1-11-10$

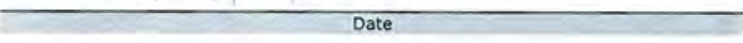

$2 / 03 / 10$ 
Data Report Form DRF-248: Compact Matrix Density

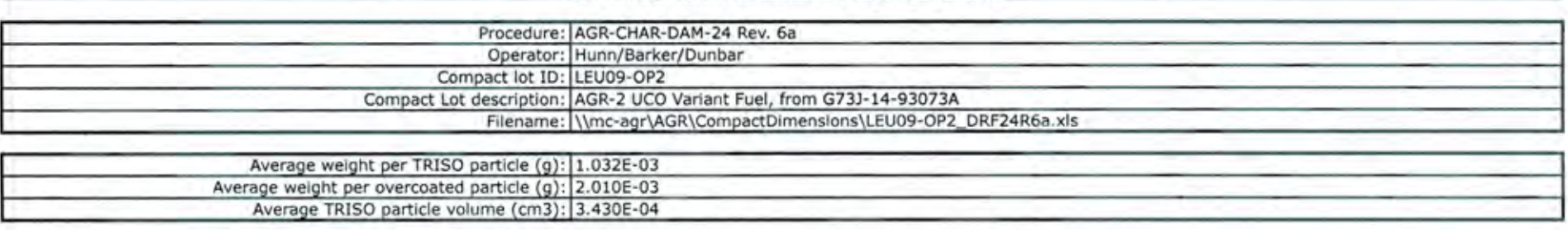
Acceptance criteria for matrix density: $\mid \geq 1.45$

\begin{tabular}{|c|c|c|c|c|c|c|c|c|c|c|}
\hline $\begin{array}{c}\text { Compact } \\
\text { ID Number }\end{array}$ & $\begin{array}{l}\text { Compact Weight } \\
\text { (a) }\end{array}$ & $\begin{array}{l}\text { Length } \\
(\mathrm{mm})\end{array}$ & $\begin{array}{l}\text { Av. Diameter } \\
\text { (mm) }\end{array}$ & $\begin{array}{c}\text { Compact Volume } \\
(\mathrm{cm} 3)\end{array}$ & $\begin{array}{l}\text { Charge Weight } \\
\text { (a) }\end{array}$ & $\begin{array}{l}\text { Particle Weight } \\
\text { (a) }\end{array}$ & $\begin{array}{l}\text { Particle Volume } \\
\text { (cm3) }\end{array}$ & Packing Fraction & $\begin{array}{l}\text { Matrix Density } \\
(\mathrm{a} / \mathrm{cm} 3)\end{array}$ & $\begin{array}{c}\text { Accept? } \\
\text { (pass or fail) }\end{array}$ \\
\hline 2051 & 6.3002 & 25.111 & 12.29 & 2.98 & 6.4303 & 3.3014 & 1.10 & $37 \%$ & 1.59 & pass \\
\hline 2052 & 6.3182 & 25.171 & 12.29 & 2.99 & 6.4315 & 3.3024 & 1.10 & $37 \%$ & 1.60 & pass \\
\hline 2053 & 6.2827 & 25.109 & 12.28 & 2.98 & 6.4321 & 3.3024 & 1.10 & $37 \%$ & 1.59 & pass \\
\hline 2054 & 6.3061 & 25.146 & 12.29 & 2.98 & 6.4316 & 3.3024 & 1.10 & $37 \%$ & 1.59 & pass \\
\hline 2055 & 6.2937 & 25.140 & 12.29 & 2.98 & 6.4321 & 3.3024 & 1.10 & $37 \%$ & 1.59 & pass \\
\hline 2056 & 6.3073 & 25.137 & 12.28 & 2.98 & 6.4316 & 3.3024 & 1.10 & $37 \%$ & 1.60 & pass \\
\hline 2057 & 6.2933 & 25.179 & 12.29 & 2.99 & 6.4309 & 3.3014 & 1.10 & $37 \%$ & 1.58 & pass \\
\hline 2058 & 6.2825 & 25.128 & 12.29 & 2.98 & 6.4295 & 3.3014 & 1.10 & $37 \%$ & 1.58 & pass \\
\hline 2059 & 6.2939 & 25.146 & 12.29 & 2.98 & 6.4307 & 3.3014 & 1.10 & $37 \%$ & 1.59 & pass \\
\hline 2060 & 6.2949 & 25.158 & 12.29 & 2.98 & 6.4308 & 3.3014 & 1.10 & $37 \%$ & 1.59 & pass \\
\hline 2061 & 6.2958 & 25.161 & 12.29 & 2.98 & 6.4323 & 3.3024 & 1.10 & $37 \%$ & 1.59 & pass \\
\hline 2062 & 6.2811 & 25.146 & 12.29 & 2.98 & 6.4306 & 3.3014 & 1.10 & $37 \%$ & 1.58 & pass \\
\hline 2063 & 6.2910 & 25.135 & 12.28 & 2.98 & 6.4314 & 3.3024 & 1.10 & $37 \%$ & 1.59 & pass \\
\hline 2064 & 6.3014 & 25.142 & 12.29 & 2.98 & 6.4314 & 3.3024 & 1.10 & $37 \%$ & 1.59 & pass \\
\hline 2065 & 6.2900 & 25.134 & 12.28 & 2.98 & 6.4321 & 3.3024 & 1.10 & $37 \%$ & 1.59 & pass \\
\hline 2066 & 6.2968 & 25.132 & 12.29 & 2.98 & 6.4300 & 3.3014 & 1.10 & $37 \%$ & 1.59 & pass \\
\hline 2067 & 6.2931 & 25.154 & 12.29 & 2.98 & 6.4311 & 3.3024 & 1.10 & $37 \%$ & 1.59 & pass \\
\hline 2068 & 6.3137 & 25.156 & 12.29 & 2.99 & 6.4325 & 3.3024 & 1.10 & $37 \%$ & 1.60 & pass \\
\hline 2069 & 6.3230 & 25.187 & 12.29 & 2.99 & 6.4325 & 3.3024 & 1.10 & $37 \%$ & 1.60 & pass \\
\hline 2070 & 6.2840 & 25.135 & 12.29 & 2.98 & 6.4324 & 3.3024 & 1.10 & $37 \%$ & 1.58 & pass \\
\hline 2071 & 6.3029 & 25.139 & 12.28 & 2.98 & 6.4326 & 3.3024 & 1.10 & $37 \%$ & 1.60 & pass \\
\hline 2072 & 6.3009 & 25.149 & 12.29 & 2.98 & 6.4323 & 3.3024 & 1.10 & $37 \%$ & 1.59 & pass \\
\hline 2073 & 6.2941 & 25.134 & 12.29 & 2.98 & 6.4311 & 3.3024 & 1.10 & $37 \%$ & 1.59 & pass \\
\hline 2074 & 6.3016 & 25.151 & 12.29 & 2.98 & 6.4308 & 3.3014 & 1.10 & $37 \%$ & 1.59 & pass \\
\hline 2075 & 6.3041 & 25.154 & 12.29 & 2.98 & 6.4309 & 3.3014 & 1.10 & $37 \%$ & 1.59 & pass \\
\hline 2076 & 6.3005 & 25.154 & 12.30 & 2.99 & 6.4331 & 3.3034 & 1.10 & $37 \%$ & 1.59 & pass \\
\hline 2077 & 6.2961 & 25.130 & 12.29 & 2.98 & 6.4319 & 3.3024 & 1.10 & $37 \%$ & 1.59 & pass \\
\hline 2078 & 6.2966 & 25.153 & 12.29 & 2.98 & 6.4323 & 3.3024 & 1.10 & $37 \%$ & 1.59 & pass \\
\hline 2079 & 6.3000 & 25.142 & 12.29 & 2.98 & 6.4323 & 3.3024 & 1.10 & $37 \%$ & 1.59 & pass \\
\hline 2080 & 6.2773 & 25.146 & 12.29 & 2.98 & 6.4322 & 3.3024 & 1.10 & $37 \%$ & 1.58 & pass \\
\hline 2081 & 6.3018 & 25.149 & 12.29 & 2.98 & 6.4314 & 3,3024 & 1.10 & $37 \%$ & 1.59 & pass \\
\hline 2082 & 6.2970 & 25.140 & 12.28 & 2.98 & 6.4313 & 3.3024 & 1.10 & $37 \%$ & 1.59 & pass \\
\hline 2083 & 6.3064 & 25.144 & 12.29 & 2.98 & 6.4302 & 3.3014 & 1.10 & $37 \%$ & 1.59 & pass \\
\hline 2084 & 6.2906 & 25.145 & 12.29 & 2.98 & 6.4325 & 3.3024 & 1.10 & $37 \%$ & 1.59 & pass \\
\hline 2085 & 6.2757 & 25.149 & 12.28 & 2.98 & 6.4303 & 3.3014 & 1.10 & $37 \%$ & 1.58 & pass \\
\hline 2086 & 6.3034 & 25.167 & 12.29 & 2.99 & 6.4322 & 3.3024 & 1.10 & $37 \%$ & 1.59 & pass \\
\hline 2087 & 6.2944 & 25.134 & 12.29 & 2.98 & 6.4328 & 3.3024 & 1.10 & $37 \%$ & 1.59 & pass \\
\hline 2088 & 6.3006 & 25.142 & 12.29 & 2.98 & 6.4320 & 3.3024 & 1.10 & $37 \%$ & 1.59 & pass \\
\hline 2089 & 6.3115 & 25.172 & 12.30 & 2.99 & 6.4304 & 3.3014 & 1.10 & $37 \%$ & 1.59 & pass \\
\hline 2090 & 6.2800 & 25.142 & 12.29 & 2.98 & 6.4321 & 3.3024 & 1.10 & $37 \%$ & 1.58 & pass \\
\hline 2091 & 6.2869 & 25.125 & 12.29 & 2.98 & 6.4302 & 3.3014 & 1.10 & $37 \%$ & 1.59 & pass \\
\hline 2092 & 6.3015 & 25.151 & 12.29 & 2.98 & 6.4317 & 3.3024 & 1.10 & $37 \%$ & 1.59 & pass \\
\hline 2093 & 6.2954 & 25.133 & 12.29 & 2.98 & 6.4310 & 3.3024 & 1.10 & $37 \%$ & 1.59 & pass \\
\hline 2094 & 6.2980 & 25.134 & 12.29 & 2.98 & 6.4314 & 3.3024 & 1.10 & $37 \%$ & 1.59 & pass \\
\hline 2095 & 6.2926 & 25.133 & 12.28 & 2.98 & 6.4306 & 3.3014 & 1.10 & $37 \%$ & 1.59 & pass \\
\hline 2096 & 6.3214 & 25.187 & 12.30 & 2.99 & 6.4322 & 3.3024 & 1.10 & $37 \%$ & 1.59 & pass \\
\hline 2097 & 6.3053 & 25.128 & 12.29 & 2.98 & 6.4321 & 3.3024 & 1.10 & $37 \%$ & 1.59 & pass \\
\hline 2098 & 6.2893 & 25.135 & 12.29 & 2.98 & 6.4316 & 3.3024 & 1.10 & $37 \%$ & 1.59 & pass \\
\hline 2099 & 6.2882 & 25.063 & 12.28 & 2.97 & 6.4321 & 3.3024 & 1.10 & $37 \%$ & 1.59 & pass \\
\hline 2100 & 6.2957 & 25.146 & 12.29 & 2.98 & 6.4321 & 3.3024 & 1.10 & $37 \%$ & 1.59 & pass \\
\hline
\end{tabular}

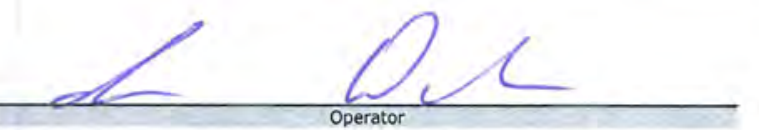

Pen

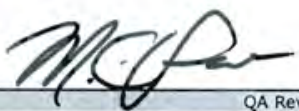

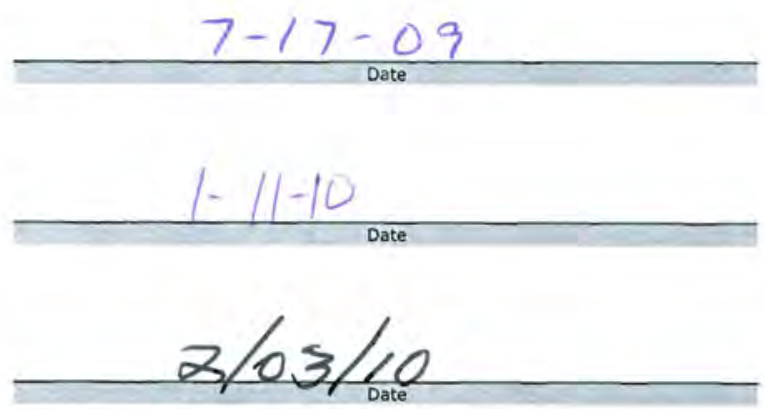


Data Report Form DRF-24B: Compact Matrix Density

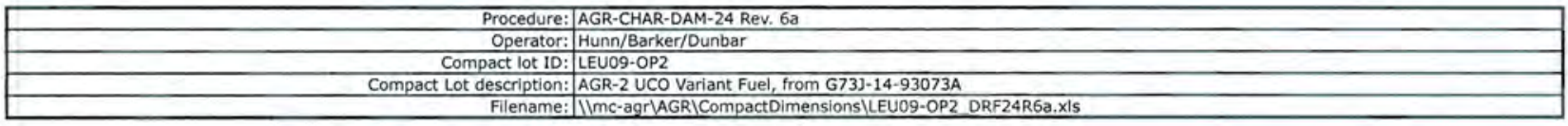

\begin{tabular}{|c|}
\hline Average weight per TRISO particle $(g): 1.032 \mathrm{E}-03$ \\
\hline Average weight per overcoated particle $(\mathrm{g}): 2.010 \mathrm{E}-03$ \\
\hline Average TRISO particle volume $(\mathrm{cm} 3): 13.430 \mathrm{E}-04$ \\
\hline
\end{tabular}

Acceptance criteria for matrix density: $\geq 1.45$

\begin{tabular}{|c|c|c|c|c|c|c|c|c|c|c|}
\hline $\begin{array}{c}\text { Compact } \\
\text { ID Number }\end{array}$ & $\begin{array}{l}\text { Compact Weight } \\
\text { (a) }\end{array}$ & $\begin{array}{l}\text { Length } \\
(\mathrm{mm})\end{array}$ & $\begin{array}{l}\text { Av. Diameter } \\
(\mathrm{mm})\end{array}$ & $\begin{array}{c}\text { Compact Volume } \\
(\mathrm{cm} 3)\end{array}$ & $\begin{array}{c}\text { Charge Weight } \\
\text { (a) }\end{array}$ & $\begin{array}{l}\text { Particle Weight } \\
\text { (a) }\end{array}$ & $\begin{array}{c}\text { Particle Volume } \\
\text { (cm } 3)\end{array}$ & Packing Fraction & $\begin{array}{l}\text { Matrix Density } \\
(\mathrm{a} / \mathrm{cm} 3)\end{array}$ & $\begin{array}{c}\text { Accept? } \\
\text { (pass or fail) }\end{array}$ \\
\hline $\mathrm{Z101}$ & 6.3040 & 25.129 & 12.29 & 2.98 & 6.4327 & 3.3024 & 1.10 & $37 \%$ & 1.59 & pass \\
\hline 2102 & 6.2944 & 25.125 & 12.29 & 2.98 & 6.4319 & 3.3024 & 1.10 & $37 \%$ & 1.59 & pass \\
\hline $\mathrm{Z103}$ & 6.2859 & 25.149 & 12.28 & 2.98 & 6.4306 & 3.3014 & 1.10 & $37 \%$ & 1.59 & pass \\
\hline $\mathrm{Z104}$ & 6.3137 & 25.153 & 12.28 & 2.98 & 6.4327 & 3.3024 & 1.10 & $37 \%$ & 1.60 & pass \\
\hline 2105 & 6.2989 & 25.115 & 12.29 & 2.98 & 6.4313 & 3.3024 & 1.10 & $37 \%$ & 1.59 & pass \\
\hline 2106 & 6.2897 & 25.120 & 12.28 & 2.98 & 6.4314 & 3.3024 & 1.10 & $37 \%$ & 1.59 & pass \\
\hline $\mathrm{Z107}$ & 6.2884 & 25.151 & 12.29 & 2.98 & 6.4299 & 3.3014 & 1.10 & $37 \%$ & 1.58 & pass \\
\hline $\mathrm{Z108}$ & 6.2840 & 25.024 & 12.28 & 2.96 & 6.4299 & 3.3014 & 1.10 & $37 \%$ & 1.60 & pass \\
\hline 2109 & 6.2941 & 25.152 & 12.29 & 2.98 & 6.4329 & 3.3024 & 1.10 & $37 \%$ & 1.59 & pass \\
\hline $\mathrm{Z110}$ & 6.2837 & 25.151 & 12.28 & 2.98 & 6.4321 & 3.3024 & 1.10 & $37 \%$ & 1.58 & pass \\
\hline$Z 111$ & 6.2904 & 25.152 & 12.29 & 2.98 & 6.4322 & 3.3024 & 1.10 & $37 \%$ & 1.59 & pass \\
\hline $\mathrm{Z} 112$ & 6.2922 & 25.134 & 12.29 & 2.98 & 6.4319 & 3.3024 & 1.10 & $37 \%$ & 1.59 & pass \\
\hline Z113 & 6.2861 & 25.114 & 12.29 & 2.98 & 6.4303 & 3.3014 & 1.10 & $37 \%$ & 1.59 & pass \\
\hline 2114 & 6.2934 & 25.130 & 12.29 & 2.98 & 6.4311 & 3.3024 & 1.10 & $37 \%$ & 1.59 & pass \\
\hline Z115 & 6.2832 & 25.132 & 12.28 & 2.98 & 6.4310 & 3.3024 & 1.10 & $37 \%$ & 1.59 & pass \\
\hline 2116 & 6.2870 & 25.142 & 12.29 & 2.98 & 6.4303 & 3.3014 & 1.10 & $37 \%$ & 1.58 & pass \\
\hline$Z 117$ & 6.3025 & 25.148 & 12.29 & 2.98 & 6.4308 & 3.3014 & 1.10 & $37 \%$ & 1.59 & pass \\
\hline $\mathrm{Z118}$ & 6.2928 & 25.151 & 12.29 & 2.98 & 6.4313 & 3.3024 & 1.10 & $37 \%$ & 1.59 & pass \\
\hline 2119 & 6.2985 & 25.158 & 12.29 & 2.98 & 6.4313 & 3.3024 & 1.10 & $37 \%$ & 1.59 & pass \\
\hline $\mathrm{Z120}$ & 6.3053 & 25.120 & 12.29 & 2.98 & 6.4320 & 3.3024 & 1.10 & $37 \%$ & 1.59 & pass \\
\hline $\mathrm{Z121}$ & 6.2959 & 25.135 & 12.29 & 2.98 & 6.4308 & 3.3014 & 1.10 & $37 \%$ & 1.59 & pass \\
\hline$Z 122$ & 6.2843 & 25.130 & 12.29 & 2.98 & 6.4309 & 3.3014 & 1.10 & $37 \%$ & 1.59 & pass \\
\hline $\mathrm{Z} 123$ & 6.2986 & 25.151 & 12.29 & 2.98 & 6.4329 & 3.3024 & 1.10 & $37 \%$ & 1.59 & pass \\
\hline$Z 124$ & 6.2830 & 25.130 & 12.29 & 2.98 & 6.4319 & 3.3024 & 1.10 & $37 \%$ & 1.58 & pass \\
\hline $\mathrm{Z125}$ & 6.3026 & 25.139 & 12.29 & 2.98 & 6.4306 & 3.3014 & 1.10 & $37 \%$ & 1.59 & pass \\
\hline $\mathrm{Z} 126$ & 6.2944 & 25.134 & 12.29 & 2.98 & 6.4309 & 3.3014 & 1.10 & $37 \%$ & 1.59 & pass \\
\hline Z127 & 6.2828 & 25.142 & 12.29 & 2.98 & 6.4327 & 3.3024 & 1.10 & $37 \%$ & 1.58 & pass \\
\hline $\mathrm{Z128}$ & 6.2886 & 25.140 & 12.29 & 2.98 & 6.4315 & 3.3024 & 1.10 & $37 \%$ & 1.59 & pass \\
\hline 2129 & 6.2837 & 25.139 & 12.29 & 2.98 & 6.4321 & 3.3024 & 1.10 & $37 \%$ & 1.58 & pass \\
\hline $\mathrm{Z} 130$ & 6.2855 & 25.132 & 12.29 & 2.98 & 6.4322 & 3.3024 & 1.10 & $37 \%$ & 1.58 & pass \\
\hline $\mathrm{Z131}$ & 6.2804 & 25.142 & 12.28 & 2.98 & 6.4323 & 3.3024 & 1.10 & $37 \%$ & 1.58 & pass \\
\hline $\mathrm{Z} 132$ & 6.3422 & 25.217 & 12.28 & 2.99 & 6.4330 & 3.3024 & 1.10 & $37 \%$ & 1.61 & pass \\
\hline 2133 & 6.2959 & 25.133 & 12.29 & 2.98 & 6.4321 & 3.3024 & 1.10 & $37 \%$ & 1.59 & pass \\
\hline$Z 134$ & 6.2983 & 25.147 & 12.29 & 2.98 & 6.4320 & 3.3024 & 1.10 & $37 \%$ & 1.59 & pass \\
\hline 2135 & 6.2972 & 25.133 & 12.28 & 2.98 & 6.4319 & 3.3024 & 1.10 & $37 \%$ & 1.59 & pass \\
\hline 2136 & 6.2922 & 25.140 & 12.29 & 2.98 & 6.4304 & 3.3014 & 1.10 & $37 \%$ & 1.59 & pass \\
\hline 2137 & 6.2877 & 25.144 & 12.29 & 2.98 & 6.4325 & 3.3024 & 1.10 & $37 \%$ & 1.58 & pass \\
\hline 2138 & 6.3043 & 25.177 & 12.29 & 2.99 & 6.4316 & 3.3024 & 1.10 & $37 \%$ & 1.59 & pass \\
\hline 2139 & 6.2862 & 25.112 & 12.28 & 2.97 & 6.4312 & 3.3024 & 1.10 & $37 \%$ & 1.59 & pass \\
\hline$Z 140$ & 6.2964 & 25.161 & 12.29 & 2.98 & 6.4318 & 3.3024 & 1.10 & $37 \%$ & 1.59 & pass \\
\hline$Z 141$ & 6.2819 & 25.132 & 12.28 & 2.98 & 6.4330 & 3.3024 & 1.10 & $37 \%$ & 1.58 & pass \\
\hline$Z 142$ & 6.2926 & 25.114 & 12.29 & 2.98 & 6.4329 & 3.3024 & 1.10 & $37 \%$ & 1.59 & pass \\
\hline 2143 & 6.2894 & 25.148 & 12.29 & 2.98 & 6.4317 & 3.3024 & 1.10 & $37 \%$ & 1.59 & pass \\
\hline 2144 & 6.2958 & 25.156 & 12.28 & 2.98 & 6.4326 & 3.3024 & 1.10 & $37 \%$ & 1.59 & pass \\
\hline$Z 145$ & 6.2905 & 25.134 & 12.29 & 2.98 & 6.4315 & 3.3024 & 1.10 & $37 \%$ & 1.59 & pass \\
\hline 2146 & 6.3008 & 25.163 & 12.29 & 2.98 & 6.4320 & 3.3024 & 1.10 & $37 \%$ & 1.59 & pass \\
\hline $\mathrm{Z} 147$ & 6.3024 & 25.158 & 12.29 & 2.98 & 6.4322 & 3.3024 & 1.10 & $37 \%$ & 1.59 & pass \\
\hline$Z 148$ & 6.2992 & 25.142 & 12.29 & 2.98 & 6.4320 & 3.3024 & 1.10 & $37 \%$ & 1.59 & pass \\
\hline 2149 & 6.2842 & 25.115 & 12.29 & 2.98 & 6.4305 & 3.3014 & 1.10 & $37 \%$ & 1.59 & pass \\
\hline 2150 & 6.2950 & 25.148 & 12.29 & 2.98 & 6.4321 & 3.3024 & 1.10 & $37 \%$ & 1.59 & pass \\
\hline
\end{tabular}

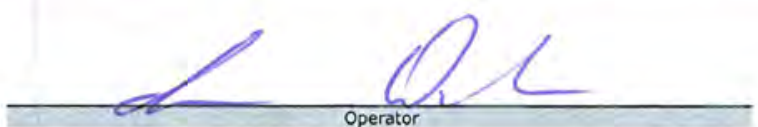

- whe 14 acsupervisor

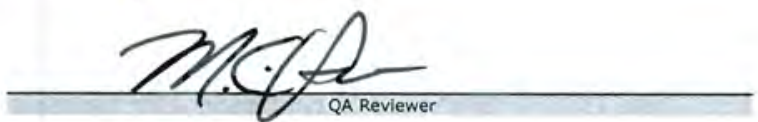

$7-17-09$

$1-11-10$

$\longrightarrow$ Date

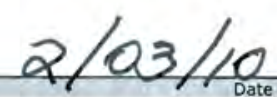


Data Report Form DRF-248: Compact Matrix Density

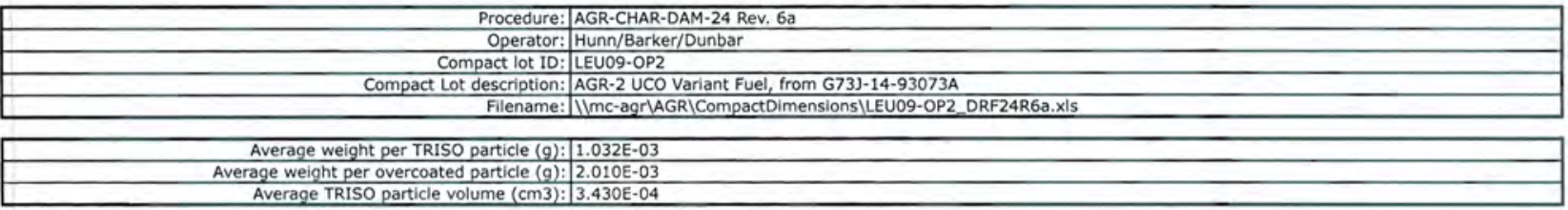

Acceptance criteria for matrix density: $\geq 1.45$

\begin{tabular}{|c|c|c|c|c|c|c|c|c|c|c|}
\hline $\begin{array}{c}\text { Compact } \\
\text { ID Number }\end{array}$ & $\begin{array}{l}\text { Compact Weight| } \\
\text { (a) }\end{array}$ & $\begin{array}{l}\text { Length } \\
(\mathrm{mm})\end{array}$ & $\begin{array}{l}\text { Av. Diameter } \\
(\mathrm{mm})\end{array}$ & \begin{tabular}{|c|} 
Compact volume \\
$(\mathrm{cm} 3)$
\end{tabular} & $\begin{array}{c}\text { Charge Weight } \\
\text { (a) }\end{array}$ & $\begin{array}{l}\text { Particle Weight } \\
\text { (a) }\end{array}$ & $\begin{array}{c}\text { Particle Volume } \\
\text { (cm3) }\end{array}$ & Packing Fraction & $\begin{array}{l}\text { Matrix Density } \\
(\mathrm{a} / \mathrm{cm} 3)\end{array}$ & $\begin{array}{c}\text { Accept? } \\
\text { (pass or fail) }\end{array}$ \\
\hline 2151 & 6.2941 & 25.156 & 12.29 & 2.98 & 6.4315 & 3.3024 & 1.10 & $37 \%$ & 1.59 & pass \\
\hline $\mathrm{Z152}$ & 6.3043 & 25.140 & 12.29 & 2.98 & 6.4319 & 3.3024 & 1.10 & $37 \%$ & 1.59 & pass \\
\hline 2153 & 6.2956 & 25.173 & 12.29 & 2.98 & 6.4305 & 3.3014 & 1.10 & $37 \%$ & 1.59 & pass \\
\hline 2154 & 6.2861 & 25.146 & 12.29 & 2.98 & 6.4311 & 3.3024 & 1.10 & $37 \%$ & 1.58 & pass \\
\hline 2155 & 6.2828 & 25.125 & 12.29 & 2.98 & 6.4305 & 3.3014 & 1.10 & $37 \%$ & 1.58 & pass \\
\hline 2156 & 6.3057 & 25.156 & 12.29 & 2.98 & 6.4330 & 3.3024 & 1.10 & $37 \%$ & 1.59 & pass \\
\hline Z157 & 6.3038 & 25.152 & 12.29 & 2.98 & 6.4315 & 3.3024 & 1.10 & $37 \%$ & 1.59 & pass \\
\hline$Z 158$ & 6.2780 & 25.133 & 12.28 & 2.98 & 6.4313 & 3.3024 & 1.10 & $37 \%$ & 1.58 & pass \\
\hline 2159 & 6.3027 & 25.132 & 12.29 & 2.98 & 6.4321 & 3.3024 & 1.10 & $37 \%$ & 1.59 & pass \\
\hline $\mathrm{Z160}$ & 6.2813 & 25.060 & 12.28 & 2.97 & 6.4320 & 3.3024 & 1.10 & $37 \%$ & 1.59 & pass \\
\hline $\mathrm{Z} 161$ & 6.2758 & 25.140 & 12.29 & 2.98 & 6.4308 & 3.3014 & 1.10 & $37 \%$ & 1.58 & pass \\
\hline $\mathrm{Z162}$ & 6.2839 & 25.125 & 12.29 & 2.98 & 6.4323 & 3.3024 & 1.10 & $37 \%$ & 1.59 & pass \\
\hline Z163 & 6.2985 & 25.142 & 12.29 & 2.98 & 6.4321 & 3.3024 & 1.10 & $37 \%$ & 1.59 & pass \\
\hline 2164 & 6.2829 & 25.048 & 12.29 & 2.97 & 6.4319 & 3.3024 & 1.10 & $37 \%$ & 1.59 & pass \\
\hline 2165 & 6.2897 & 25.116 & 12.28 & 2.97 & 6.4317 & 3.3024 & 1.10 & $37 \%$ & 1.59 & pass \\
\hline 2166 & 6.2864 & 25.161 & 12.29 & 2.98 & 6.4303 & 3.3014 & 1.10 & $37 \%$ & 1.58 & pass \\
\hline 2167 & 6.2828 & 25.168 & 12.29 & 2.98 & 6.4315 & 3.3024 & 1.10 & $37 \%$ & 1.58 & pass \\
\hline 2168 & & & & & & & & & & \\
\hline 2169 & & & & & & & & & & \\
\hline 2170 & & & & & & & & & & \\
\hline 2171 & & & & & & & & & & \\
\hline $\mathrm{Z} 172$ & & & & & & & & & & \\
\hline $\mathrm{z} 173$ & & & & & & & & & & \\
\hline $\mathrm{z} 174$ & & & & & & & & & & \\
\hline 2175 & & & & & & & & & & \\
\hline 2176 & & & & & & & & & & \\
\hline 2177 & & & & & & & & & & \\
\hline 2178 & & & & & & & & & & \\
\hline Z179 & & & & & & & & & & \\
\hline 2180 & & & & & & & & & & \\
\hline 2181 & & & & & & & & & & \\
\hline 2182 & & & & & & & & & & \\
\hline $\mathrm{Z} 183$ & & & & & & & & & & \\
\hline $\mathrm{Z184}$ & & & & & & & & & & \\
\hline 2185 & & & & & & & & & & \\
\hline 2186 & & & & & & & & & & \\
\hline 2187 & & & & & & & & & & \\
\hline 2188 & & & & & & & & & & \\
\hline 2189 & & & & & & & & & & \\
\hline Z190 & & & & & & & & & & \\
\hline Z191 & & & & & & & & & & \\
\hline Z192 & & & & & & & & & & \\
\hline Z193 & & & & & & & & & & \\
\hline 2194 & & & & & & & & & & \\
\hline $\mathrm{Z195}$ & & & & & & & & & & \\
\hline Z196 & & & & & & & & & & \\
\hline 2197 & & & & & & & & & & \\
\hline Z198 & & & & & & & & & & \\
\hline Z199 & & & & & & & & & & \\
\hline 2200 & & & & & & & & & & \\
\hline
\end{tabular}

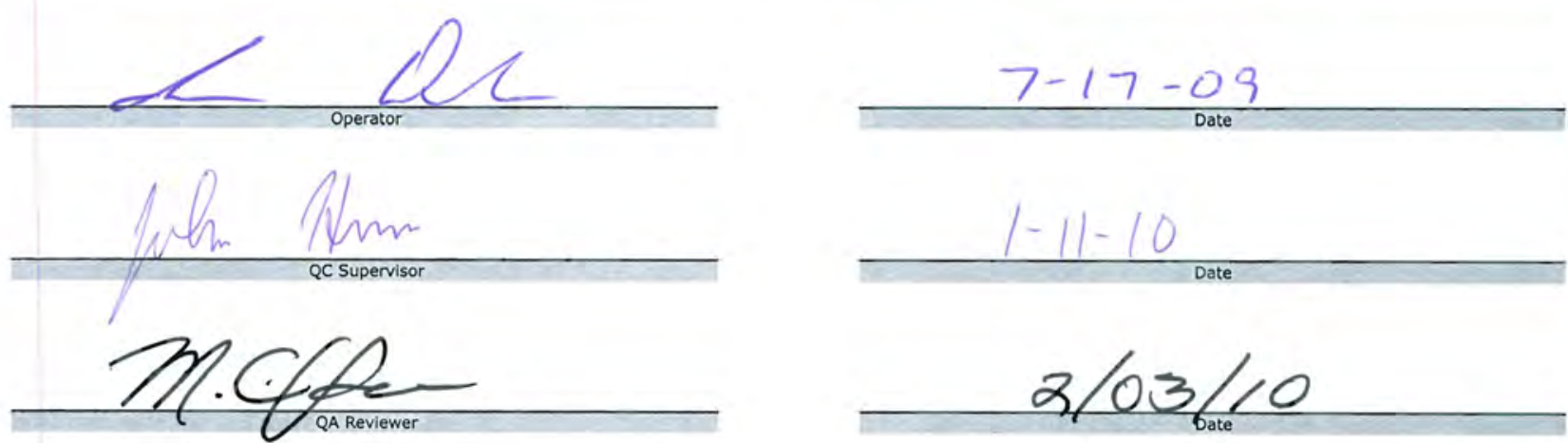


Data Report Form DRF-24C: Compact Tracking

\begin{tabular}{|r|l|}
\hline Procedure: & AGR-CHAR-DAM-24 Rev. 6a \\
\hline Operator: & Hunn/Barker/Dunbar \\
\hline Compact lot ID: & LEU09-OP2 \\
\hline Compact Lot description: & AGR-2 UCO Variant Fuel, from G73J-14-93073A \\
\hline Filename: & IImc-agrVAGRICompactDimensions \LEU09-0P2_DRF24R6a.xIs \\
\hline
\end{tabular}

\begin{tabular}{|c|c|}
\hline $\begin{array}{l}\text { Compact } \\
\text { Z Number }\end{array}$ & $\begin{array}{c}\text { Compact G } \\
\text { Number }\end{array}$ \\
\hline 2001 & G094 \\
\hline 2002 & G019 \\
\hline 2003 & G041 \\
\hline 2004 & G087 \\
\hline$Z 005$ & G153 \\
\hline 2006 & G008 \\
\hline 2007 & G111 \\
\hline$Z 008$ & G039 \\
\hline 2009 & G053 \\
\hline 2010 & G118 \\
\hline Z011 & G096 \\
\hline 2012 & G082 \\
\hline Z013 & G069 \\
\hline Z014 & $\mathrm{G} 026$ \\
\hline Z015 & G006 \\
\hline 2016 & G047 \\
\hline Z017 & $\mathrm{G} 048$ \\
\hline Z018 & G046 \\
\hline 2019 & G061 \\
\hline 2020 & G080 \\
\hline 2021 & $\mathrm{G} 036$ \\
\hline 2022 & G135 \\
\hline 2023 & G085 \\
\hline 2024 & G099 \\
\hline 2025 & G052 \\
\hline 2026 & G109 \\
\hline$\overline{Z O 27}$ & G060 \\
\hline 2028 & G163 \\
\hline 2029 & G114 \\
\hline 2030 & G075 \\
\hline 2031 & G009 \\
\hline 2032 & G027 \\
\hline Z033 & G148 \\
\hline 2034 & G050 \\
\hline 2035 & G015 \\
\hline 2036 & G156 \\
\hline 2037 & G058 \\
\hline 2038 & G044 \\
\hline 2039 & G074 \\
\hline 2040 & G162 \\
\hline$Z 041$ & G185 \\
\hline 2042 & G097 \\
\hline Z043 & G175 \\
\hline Z044 & G176 \\
\hline 2045 & G049 \\
\hline 2046 & $\mathrm{G} 062$ \\
\hline 2047 & G142 \\
\hline 2048 & G157 \\
\hline 2049 & G012 \\
\hline$Z 050$ & G145 \\
\hline
\end{tabular}

\begin{tabular}{|c|c|}
\hline $\begin{array}{l}\text { Compact } \\
Z \text { Number }\end{array}$ & $\begin{array}{c}\text { Compact G } \\
\text { Number }\end{array}$ \\
\hline Z051 & G116 \\
\hline Z052 & G083 \\
\hline 2053 & $\mathrm{G} 013$ \\
\hline Z054 & G107 \\
\hline 2055 & G121 \\
\hline Z056 & G077 \\
\hline 2057 & G086 \\
\hline Z058 & G138 \\
\hline 2059 & G134 \\
\hline$Z 060$ & G144 \\
\hline Z061 & G167 \\
\hline Z062 & G028 \\
\hline Z063 & G139 \\
\hline Z064 & G057 \\
\hline Z065 & G173 \\
\hline Z066 & G098 \\
\hline 2067 & G120 \\
\hline 2068 & G079 \\
\hline 2069 & G081 \\
\hline$Z 070$ & G035 \\
\hline$Z 071$ & G093 \\
\hline 2072 & G181 \\
\hline 2073 & G143 \\
\hline 2074 & G051 \\
\hline Z075 & G122 \\
\hline 2076 & G178 \\
\hline Z077 & G113 \\
\hline Z078 & G126 \\
\hline 2079 & G070 \\
\hline 2080 & G032 \\
\hline 2081 & G182 \\
\hline 2082 & G154 \\
\hline 2083 & G076 \\
\hline Z084 & G152 \\
\hline Z085 & G011 \\
\hline Z086 & G059 \\
\hline 2087 & G117 \\
\hline 2088 & G124 \\
\hline 2089 & G063 \\
\hline$Z 090$ & $\mathrm{G} 040$ \\
\hline 2091 & G140 \\
\hline Z092 & G184 \\
\hline$Z 093$ & G123 \\
\hline$Z 094$ & G055 \\
\hline$Z 095$ & G067 \\
\hline Z096 & G084 \\
\hline Z097 & G177 \\
\hline Z098 & $\mathrm{G} 042$ \\
\hline ZOO9 & G007 \\
\hline 2100 & G056 \\
\hline
\end{tabular}

\begin{tabular}{|c|c|}
\hline $\begin{array}{l}\text { Compact } \\
\text { Z Number }\end{array}$ & $\begin{array}{c}\text { Compact G } \\
\text { Number }\end{array}$ \\
\hline $\mathrm{Z101}$ & G179 \\
\hline$Z 102$ & G158 \\
\hline$Z 103$ & G131 \\
\hline Z104 & G183 \\
\hline Z105 & G092 \\
\hline Z106 & G091 \\
\hline Z107 & G166 \\
\hline Z108 & 6005 \\
\hline Z109 & G141 \\
\hline$Z 110$ & G129 \\
\hline Z111 & G132 \\
\hline$Z 112$ & G017 \\
\hline$Z 113$ & G029 \\
\hline$Z 114$ & G164 \\
\hline Z115 & G095 \\
\hline Z116 & G160 \\
\hline Z117 & G165 \\
\hline Z118 & G168 \\
\hline Z119 & G102 \\
\hline$Z 120$ & G174 \\
\hline Z121 & G103 \\
\hline$Z 122$ & G030 \\
\hline Z123 & G101 \\
\hline Z124 & G018 \\
\hline Z125 & G110 \\
\hline Z126 & G125 \\
\hline Z127 & G038 \\
\hline Z128 & G151 \\
\hline Z129 & G172 \\
\hline$Z 130$ & G064 \\
\hline Z131 & G025 \\
\hline Z132 & G045 \\
\hline Z133 & G169 \\
\hline 2134 & G106 \\
\hline$Z 135$ & G073 \\
\hline $\mathrm{Z} 136$ & G170 \\
\hline Z137 & G171 \\
\hline Z138 & G078 \\
\hline Z139 & G066 \\
\hline $\mathrm{Z} 140$ & G180 \\
\hline Z141 & G136 \\
\hline Z142 & G037 \\
\hline $\mathrm{Z143}$ & G043 \\
\hline Z144 & G071 \\
\hline Z145 & G054 \\
\hline Z146 & G128 \\
\hline Z147 & G112 \\
\hline Z148 & G090 \\
\hline Z149 & G150 \\
\hline 2150 & G119 \\
\hline
\end{tabular}

\begin{tabular}{|c|c|}
\hline $\begin{array}{l}\text { Compact } \\
\text { Z Number }\end{array}$ & $\begin{array}{c}\text { Compact G } \\
\text { Number }\end{array}$ \\
\hline Z151 & G133 \\
\hline$Z 152$ & G100 \\
\hline Z153 & G108 \\
\hline Z154 & G115 \\
\hline Z155 & G161 \\
\hline Z156 & G072 \\
\hline Z157 & G149 \\
\hline Z158 & G137 \\
\hline Z159 & G127 \\
\hline $\mathrm{Z160}$ & G004 \\
\hline Z161 & G010 \\
\hline Z162 & G014 \\
\hline $\mathrm{Z163}$ & G088 \\
\hline Z164 & G001 \\
\hline Z165 & G089 \\
\hline Z166 & G159 \\
\hline$Z 167$ & G031 \\
\hline$Z 168$ & \\
\hline Z169 & \\
\hline 2170 & \\
\hline Z171 & \\
\hline $\mathrm{Z172}$ & \\
\hline 2173 & \\
\hline $\mathrm{Z} 174$ & \\
\hline$Z 175$ & \\
\hline$Z 176$ & \\
\hline$Z 177$ & \\
\hline Z178 & \\
\hline Z179 & \\
\hline $\mathrm{Z180}$ & \\
\hline$Z 181$ & \\
\hline$Z 182$ & \\
\hline 2183 & \\
\hline Z184 & \\
\hline Z185 & \\
\hline 2186 & \\
\hline Z187 & \\
\hline Z188 & \\
\hline 2189 & \\
\hline $\mathrm{Z190}$ & \\
\hline Z191 & \\
\hline Z192 & \\
\hline Z193 & \\
\hline Z194 & \\
\hline $\mathrm{Z195}$ & \\
\hline$Z 196$ & \\
\hline Z197 & \\
\hline$Z 198$ & \\
\hline Z199 & \\
\hline 2200 & \\
\hline
\end{tabular}

\begin{tabular}{|c|c|}
\hline $\begin{array}{l}\text { Compact } \\
\text { Z Number }\end{array}$ & $\begin{array}{l}\text { Compact G } \\
\text { Number }\end{array}$ \\
\hline \multicolumn{2}{|l|}{2201} \\
\hline \multicolumn{2}{|l|}{2202} \\
\hline \multicolumn{2}{|l|}{2203} \\
\hline \multicolumn{2}{|l|}{ Z204 } \\
\hline \multicolumn{2}{|l|}{2205} \\
\hline \multicolumn{2}{|l|}{ Z206 } \\
\hline \multicolumn{2}{|l|}{ Z207 } \\
\hline \multicolumn{2}{|l|}{$Z 208$} \\
\hline \multicolumn{2}{|l|}{2209} \\
\hline \multicolumn{2}{|l|}{$\frac{2210}{7211}$} \\
\hline \multicolumn{2}{|l|}{2211} \\
\hline \multicolumn{2}{|l|}{$Z 212$} \\
\hline \multicolumn{2}{|l|}{2213} \\
\hline \multicolumn{2}{|l|}{2214} \\
\hline \multicolumn{2}{|l|}{$\frac{Z 215}{Z 216}$} \\
\hline \multicolumn{2}{|l|}{2216} \\
\hline \multicolumn{2}{|l|}{ Z217 } \\
\hline \multicolumn{2}{|l|}{$Z 218$} \\
\hline \multicolumn{2}{|l|}{$Z 219$} \\
\hline \multicolumn{2}{|l|}{2220} \\
\hline \multicolumn{2}{|l|}{2221} \\
\hline \multicolumn{2}{|l|}{ Z222 } \\
\hline \multicolumn{2}{|l|}{2223} \\
\hline Z224 & \\
\hline$Z 225$ & \\
\hline Z226 & \\
\hline 2227 & \\
\hline$Z 228$ & \\
\hline$Z 229$ & \\
\hline$Z 230$ & \\
\hline Z231 & \\
\hline 2232 & \\
\hline 2233 & \\
\hline Z234 & \\
\hline Z235 & \\
\hline 2236 & \\
\hline Z237 & \\
\hline$Z 238$ & \\
\hline 2239 & \\
\hline 2240 & \\
\hline 2241 & \\
\hline 2242 & \\
\hline$Z 243$ & \\
\hline 2244 & \\
\hline 2245 & \\
\hline$Z 246$ & \\
\hline 2247 & \\
\hline 2248 & \\
\hline$Z 249$ & \\
\hline$Z 250$ & \\
\hline
\end{tabular}

Comments

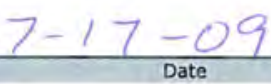


Data Report Form DRF-24D: Compact Charge Weight

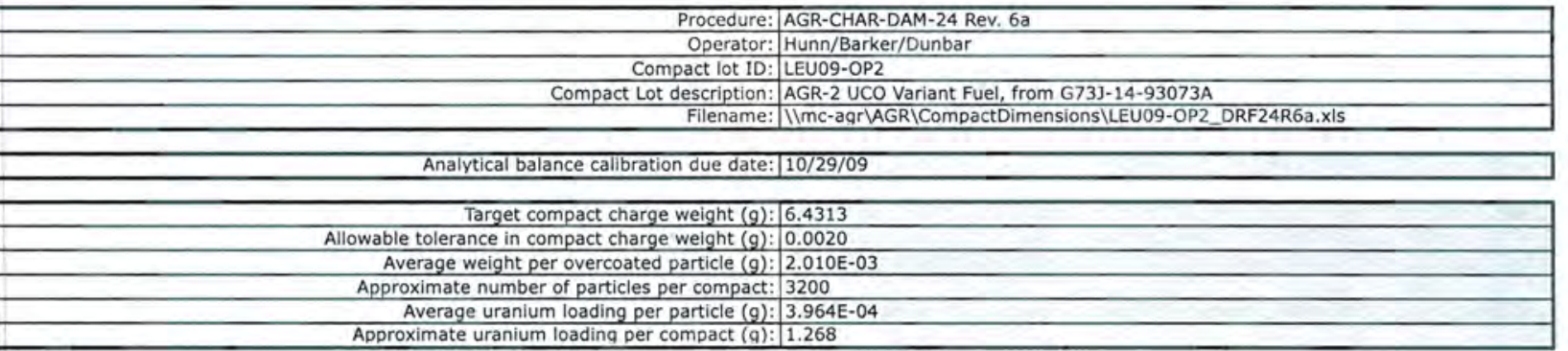

\begin{tabular}{|c|c|}
\hline $\begin{array}{c}\text { Compact } \\
\text { G Number }\end{array}$ & $\begin{array}{c}\text { Charge Weight } \\
\text { (q) }\end{array}$ \\
\hline G001 & 6.4319 \\
\hline G002 & 6.4322 \\
\hline G003 & 6.4309 \\
\hline G004 & 6.4320 \\
\hline G005 & 6.4299 \\
\hline G006 & 6.4325 \\
\hline G007 & 6.4321 \\
\hline G008 & 6.4313 \\
\hline G009 & 6.4321 \\
\hline G010 & 6.4308 \\
\hline G011 & 6.4303 \\
\hline G012 & 6.4333 \\
\hline G013 & 6.4321 \\
\hline G014 & 6.4323 \\
\hline G015 & 6.4310 \\
\hline G016 & 6.4304 \\
\hline G017 & 6.4319 \\
\hline G018 & 6.4319 \\
\hline G019 & 6.4306 \\
\hline G020 & 6.4307 \\
\hline G021 & 6.4300 \\
\hline G022 & 6.4309 \\
\hline G023 & 6.4316 \\
\hline G024 & 6.4303 \\
\hline G025 & 6.4323 \\
\hline G026 & 6.4311 \\
\hline G027 & 6.4308 \\
\hline G028 & 6.4306 \\
\hline G029 & 6.4303 \\
\hline G030 & 6.4309 \\
\hline G031 & 6.4315 \\
\hline G032 & 6.4322 \\
\hline G033 & 6.4307 \\
\hline G034 & 6.4325 \\
\hline G035 & 6.4324 \\
\hline G036 & 6.4323 \\
\hline G037 & 6.4329 \\
\hline G038 & 6.4327 \\
\hline G039 & 6.4318 \\
\hline G040 & 6.4321 \\
\hline G041 & 6.4303 \\
\hline G042 & 6.4316 \\
\hline G043 & 6.4317 \\
\hline G044 & 6.4326 \\
\hline G045 & 6.4330 \\
\hline G046 & 6.4329 \\
\hline G048 & 6.4315 \\
\hline & 6.4322 \\
\hline & 6.4325 \\
\hline
\end{tabular}

\begin{tabular}{|c|c|}
\hline $\begin{array}{c}\text { Compact } \\
\text { G Number }\end{array}$ & $\begin{array}{c}\text { Charge Weight } \\
(\mathrm{q})\end{array}$ \\
\hline G051 & 6.4308 \\
\hline G052 & 6.4302 \\
\hline G053 & 6.4326 \\
\hline G054 & 6.4315 \\
\hline G055 & 6.4314 \\
\hline G056 & 6.4321 \\
\hline G057 & 6.4314 \\
\hline G058 & 6.4309 \\
\hline G059 & 6.4322 \\
\hline G060 & 6.4320 \\
\hline G061 & 6.4325 \\
\hline G062 & 6.4311 \\
\hline G063 & 6.4304 \\
\hline G064 & 6.4322 \\
\hline G065 & 6.4319 \\
\hline G066 & 6.4312 \\
\hline G067 & 6.4306 \\
\hline G068 & 6.4308 \\
\hline G069 & 6.4313 \\
\hline G070 & 6.4323 \\
\hline G071 & 6.4326 \\
\hline G072 & 6.4330 \\
\hline G073 & 6.4319 \\
\hline G074 & 6.4310 \\
\hline G075 & 6.4304 \\
\hline G076 & 6.4302 \\
\hline G077 & 6.4316 \\
\hline G078 & 6.4316 \\
\hline G079 & 6.4325 \\
\hline G080 & 6.4312 \\
\hline G081 & 6.4325 \\
\hline G082 & 6.4329 \\
\hline G083 & 6.4315 \\
\hline G084 & 6.4322 \\
\hline G085 & 6.4314 \\
\hline G086 & 6.4309 \\
\hline G087 & 6.4318 \\
\hline G088 & 6.4321 \\
\hline G089 & 6.4317 \\
\hline G090 & 6.4320 \\
\hline G091 & 6.4314 \\
\hline G092 & 6.4313 \\
\hline G093 & 6.4326 \\
\hline G094 & 6.4327 \\
\hline G095 & 6.4310 \\
\hline G096 & 6.4307 \\
\hline G097 & 6.4329 \\
\hline G098 & 6.4300 \\
\hline G100 & 6.4302 \\
\hline 6.4319 \\
\hline
\end{tabular}

\begin{tabular}{|c|c|}
\hline $\begin{array}{c}\text { Compact } \\
\text { G Number }\end{array}$ & $\begin{array}{c}\text { Charge Weight } \\
\text { (a) }\end{array}$ \\
\hline G101 & 6.4329 \\
\hline G102 & 6.4313 \\
\hline G103 & 6.4308 \\
\hline G104 & 6.4311 \\
\hline G105 & 6.4326 \\
\hline G106 & 6.4320 \\
\hline G107 & 6.4316 \\
\hline G108 & 6.4305 \\
\hline G109 & 6.4314 \\
\hline G110 & 6.4306 \\
\hline G111 & 6.4311 \\
\hline G112 & 6.4322 \\
\hline G113 & 6.4319 \\
\hline G114 & 6.4328 \\
\hline G115 & 6.4311 \\
\hline G116 & 6.4303 \\
\hline G117 & 6.4328 \\
\hline G118 & 6.4322 \\
\hline G119 & 6.4321 \\
\hline G120 & 6.4311 \\
\hline G121 & 6.4321 \\
\hline G122 & 6.4309 \\
\hline G123 & 6.4310 \\
\hline G124 & 6.4320 \\
\hline G125 & 6.4309 \\
\hline G126 & 6.4323 \\
\hline G127 & 6.4321 \\
\hline G128 & 6.4320 \\
\hline G129 & 6.4321 \\
\hline G130 & 6.4314 \\
\hline G131 & 6.4306 \\
\hline G132 & 6.4322 \\
\hline G133 & 6.4315 \\
\hline G134 & 6.4307 \\
\hline G135 & 6.4311 \\
\hline G136 & 6.4330 \\
\hline G137 & 6.4313 \\
\hline G138 & 6.4295 \\
\hline G139 & 6.4314 \\
\hline G140 & 6.4302 \\
\hline G141 & 6.4329 \\
\hline G142 & 6.4316 \\
\hline G143 & 6.4311 \\
\hline G144 & 6.4308 \\
\hline G145 & 6.4318 \\
\hline G146 & 6.4301 \\
\hline G147 148 & 6.4325 \\
\hline G150 & 6.4326 \\
\hline & 6.4315 \\
\hline & 6.4305 \\
\hline
\end{tabular}

\begin{tabular}{|c|c|}
\hline $\begin{array}{c}\text { Compact } \\
\text { G Number }\end{array}$ & $\begin{array}{c}\text { Charge Weight } \\
\text { (a) }\end{array}$ \\
\hline G151 & 6.4315 \\
\hline G152 & 6.4325 \\
\hline G153 & 6.4309 \\
\hline G154 & 6.4313 \\
\hline G155 & 6.4311 \\
\hline G156 & 6.4326 \\
\hline G157 & 6.4306 \\
\hline G158 & 6.4319 \\
\hline G159 & 6.4303 \\
\hline G160 & 6.4303 \\
\hline G161 & 6.4305 \\
\hline G162 & 6.4327 \\
\hline G163 & 6.4320 \\
\hline G164 & 6.4311 \\
\hline G165 & 6.4308 \\
\hline G167 & 6.4299 \\
\hline G168 & 6.4323 \\
\hline G169 & 6.4313 \\
\hline G170 & 6.4321 \\
\hline G171 & 6.4304 \\
\hline G172 & 6.4325 \\
\hline G173 & 6.4321 \\
\hline G174 & 6.4321 \\
\hline G175 & 6.4320 \\
\hline G176 & 6.4309 \\
\hline G177 & 6.4321 \\
\hline G178 & 6.4331 \\
\hline G179 & 6.4327 \\
\hline G180 & 6.4318 \\
\hline G181 & 6.4323 \\
\hline G182 & 6.4314 \\
\hline G183 & 6.4327 \\
\hline G184 & 6.4317 \\
\hline G185 & 6.4304 \\
\hline G186 & \\
\hline G187 & \\
\hline G188 & \\
\hline G189 & \\
\hline G190 & \\
\hline G191 & \\
\hline G192 & \\
\hline G193 & \\
\hline G194 & \\
\hline G196 & \\
\hline G198 & \\
\hline & \\
\hline & \\
\hline & \\
\hline & \\
\hline & \\
\hline & \\
\hline & \\
\hline & \\
\hline & \\
\hline
\end{tabular}

\begin{tabular}{|c|c|}
\hline $\begin{array}{l}\text { Compact } \\
\text { G Number }\end{array}$ & $\begin{array}{c}\text { Charge } \\
\text { Weight (q) }\end{array}$ \\
\hline G201 & \\
\hline G202 & \\
\hline G203 & \\
\hline G204 & \\
\hline G205 & \\
\hline G206 & 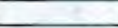 \\
\hline G207 & \\
\hline G208 & \\
\hline G209 & \\
\hline $\mathrm{G} 210$ & \\
\hline G211 & \\
\hline G212 & \\
\hline G213 & \\
\hline G214 & \\
\hline G215 & \\
\hline G216 & \\
\hline G217 & \\
\hline G218 & \\
\hline G219 & \\
\hline G220 & \\
\hline G221 & \\
\hline G222 & \\
\hline G223 & \\
\hline G224 & \\
\hline G225 & \\
\hline G226 & \\
\hline G227 & \\
\hline G228 & \\
\hline G229 & \\
\hline G230 & \\
\hline G231 & \\
\hline G232 & \\
\hline G233 & \\
\hline G234 & \\
\hline G235 & \\
\hline G236 & \\
\hline G237 & \\
\hline G238 & \\
\hline G239 & \\
\hline G240 & \\
\hline G241 & \\
\hline G242 & \\
\hline G243 & \\
\hline G244 & \\
\hline G245 & \\
\hline G246 & \\
\hline G247 & \\
\hline G248 & \\
\hline G249 & \\
\hline G250 & \\
\hline
\end{tabular}

Comments

Average weight per overcoated particle from combined results of 2 independent measurements (W09060801 and W09060802).

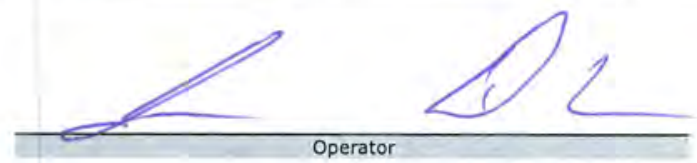

$$
6-23-0 \%
$$


Data Report Form DRF-25: Fuel Compact Mean Uranium Loading

\begin{tabular}{|r|l|}
\hline Procedure: & AGR-CHAR-DAM-25 Rev. 2 \\
\hline Operator: & Montgomery \\
\hline Compact lot ID: & LEU09-OP2-Z \\
\hline Compact lot description: & AGR-2 UCO Variant Fuel, from G73J-14-93073A \\
\hline Filename: & IImC-agr|AGRIUraniumLoading\LEU09-OP2-Z_DRF25R2.xIS \\
\hline
\end{tabular}

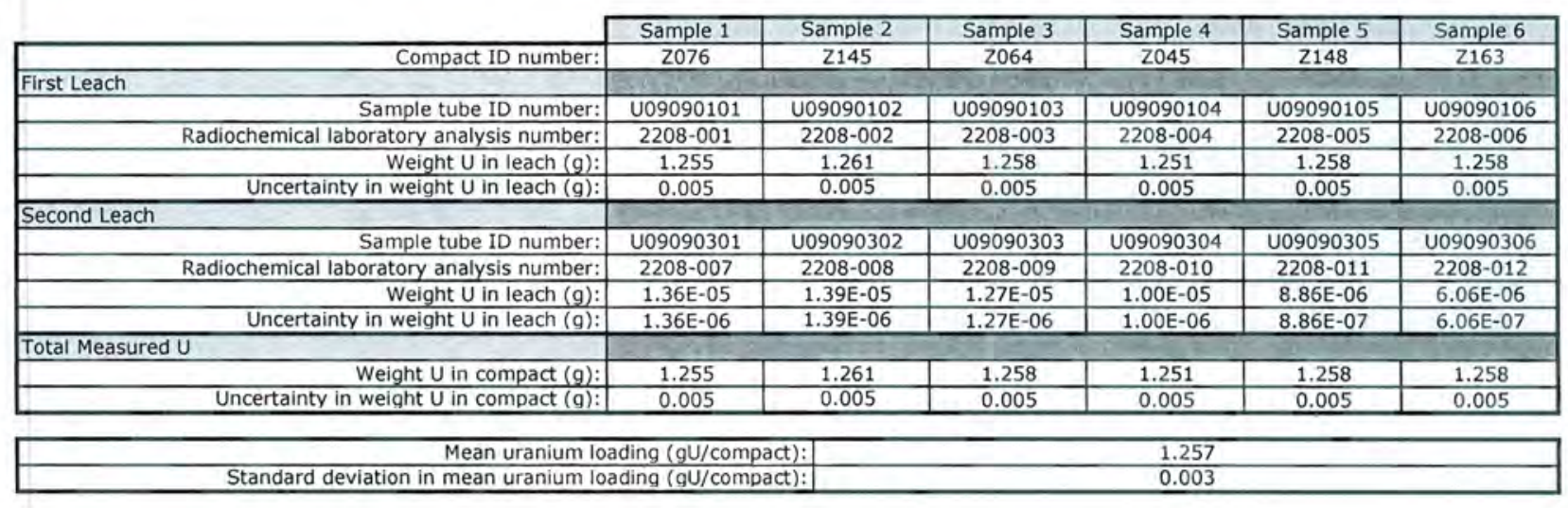

\section{Comments}

Leach 1 was analyzed by Davies-Gray titration method. Leach 2 was analyzed by ICP-MS due to low U concentration.

Davies Gray: Initial known U recovery $=100.37 \%$; Final Known U recovery $=100.13 \%$; Blind titration U recovery $=100.19 \%$

Uncertainty in Davies-Gray $(0.4 \%)$ based on average of measured \% recovery data for LEU06,07,08,09.

Isotopic composition $(w t \%): 234 \mathrm{U}=0.148 \pm 0.001 ; 235 \mathrm{U}=13.993 \pm 0.017 ; 236 \mathrm{U}=0.0504 \pm 0.0004 ; 238 \mathrm{U}=85.809 \pm 0.018$

Data checked against official results of analyses for RMAL2208 by FCM on 11/12/2009

$\frac{\text { Fud C. montgomemy }}{\text { Operator }}$


Data Report Form DRF-26A: Measurement of U Contamination or Impurities by Deconsolidation Leach

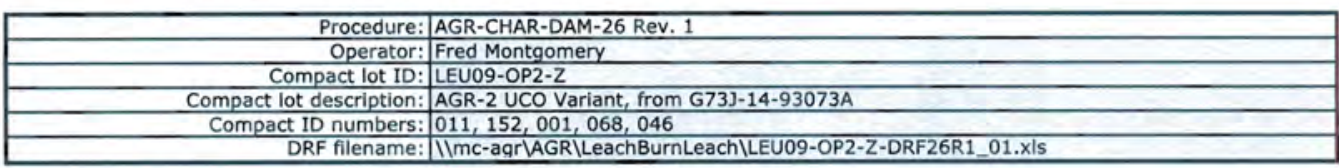

\begin{tabular}{|c|c|c|c|c|}
\hline \multicolumn{2}{|r|}{$\begin{array}{c}\text { Mean average weight uranium per particle }(g) \\
\text { Uncertainty in mean averaqe weight uranium per particle }(q):\end{array}$} & \multicolumn{3}{|c|}{$\frac{3.964 \mathrm{E}-04}{8.1 \mathrm{E}-07}$} \\
\hline & & First Leach & Second Leach & Total \\
\hline & Deconsolidation-leach solution ID: & L09080301 & L09080501 & \\
\hline & Number of compacts: & & & \\
\hline & Total volume of leach solution (mI): & 134.0 & 126.0 & \\
\hline & Radiochemical laboratory analysis number: & $2134-001$ & $2134-006$ & \\
\hline & Measured uranium concentration $(\mathrm{\mu g} / \mathrm{ml})$ : & $7.35 \mathrm{E}-02$ & $8.42 \mathrm{E}-03$ & \\
\hline & Uncertainty in uranium concentration $(\mathrm{\mu g} / \mathrm{ml})$ : & $7.35 \mathrm{E}-03$ & $8.42 \mathrm{E}-04$ & \\
\hline & $\begin{array}{c}\text { Weight uranium leached }(g): \\
\end{array}$ & $9.85 \mathrm{E}-06$ & $1.06 \mathrm{E}-06$ & $1.09 \mathrm{E}-05$ \\
\hline & Uncertainty in weight uranium leached $(g)$ : & $9.86 \mathrm{E}-07$ & $1.06 \mathrm{E}-07$ & $9.92 \mathrm{E}-07$ \\
\hline & Effective number of exposed kernels: & 0.0 & 0.0 & 0.0 \\
\hline & Uncertainty in effective number of exposed kernels: & 0.0 & 0.0 & 0.0 \\
\hline \multirow{5}{*}{$\mathrm{Fe}$} & Measured concentration of impurity in sample $(\mathrm{\mu g} / \mathrm{ml})$ : & $<4.12 \mathrm{E}-02$ & $<4.12 \mathrm{E}-02$ & $\mathrm{~F}$ \\
\hline & Uncorrected weight of impurity in sample $(\mu g)$ : & $<5.52$ & $<5.19$ & $<10.71$ \\
\hline & Weight of impurity in blank $(\mu g)$ : & $<6.55$ & $<5.07$ & \\
\hline & Minimum corrected weight of impurity in sample $(\mu g)$ : & 0.00 & 0.00 & 0.00 \\
\hline & Maximum corrected weight of impurity in sample $(\mu g)$ : & 5.52 & 5.19 & 10.71 \\
\hline \multirow{5}{*}{$\mathbf{C r}$} & Measured concentration of impurity in sample $(\mu \mathrm{g} / \mathrm{ml})$ : & $7.22 \mathrm{E}-03$ & $3.85 E-03$ & $\mathrm{Cr}$ \\
\hline & Uncorrected weight of impurity in sample $(\mu \mathrm{g})$ : & 0.97 & 0.49 & 1.45 \\
\hline & Weight of impurity in blank $(\mu g)$ : & $<0.32$ & $<0.25$ & \\
\hline & Minimum corrected weight of Impurity in sample ( $\mu \mathrm{g})$ : & 0.65 & 0.24 & 0.89 \\
\hline & Maximum corrected weight of impurity in sample $(\mu g)$ : & 0.97 & 0.49 & 1.45 \\
\hline \multirow{5}{*}{ Mn } & Measured concentration of impurity in sample $(\mu \mathrm{g} / \mathrm{ml})$ : & $<1.91 \mathrm{E}-03$ & $<1.91 \mathrm{E}-03$ & $\mathrm{Mn}$ \\
\hline & Uncorrected weight of impurity in sample $(\mu g)$ : & $<0.26$ & $<0.24$ & $<0.50$ \\
\hline & Weight of impurity in blank $(\mu g)$ : & $<0.30$ & $<0.23$ & \\
\hline & Minimum corrected weight of impurity in sample ( $\mu \mathrm{g})$ : & 0.00 & 0.00 & 0.00 \\
\hline & Maximum corrected weight of impurity in sample $(\mu \mathrm{g})$ : & 0.26 & 0.24 & 0.50 \\
\hline \multirow{4}{*}{ co } & Measured concentration of impurity in sample $(\mu \mathrm{g} / \mathrm{ml})$ : & $<1.62 \mathrm{E}-03$ & $<1.62 \mathrm{E}-03$ & Co \\
\hline & Uncorrected weight of impurity in sample $(\mu \mathrm{g})$ : & $<0.22$ & $<0.20$ & $<0.42$ \\
\hline & Weight of impurity in blank $(\mu \mathrm{g})$ : & $<0.26$ & $<0.20$ & \\
\hline & Minimum corrected weight of Impurity in sample $(\mu \mathrm{g})$ : & 0.00 & 0.00 & 0.00 \\
\hline \multirow{5}{*}{ Ni } & $\begin{array}{l}\text { Maximum corrected weight of impurity in sample }(\mu \mathrm{g}) \text { : } \\
\text { Measured concentration of impurity in sample }(\mu \mathrm{mg} / \mathrm{ml} \text { : }\end{array}$ & 0.22 & 0.20 & 0.42 \\
\hline & & $\frac{<8.00 E-03}{<1.07}$ & $\frac{<8.00 \mathrm{E}-03}{<1.01}$ & $\mathrm{Ni}$ \\
\hline & $\begin{array}{r}\text { Uncorrected weight of impurity in sample }(\mu \mathrm{g}) \text { : } \\
\text { Weight of impurity in blank }(\mu \mathrm{g}) \text { : }\end{array}$ & $\frac{<1.27}{<1.27}$ & $\frac{<1.01}{<0.98}$ & $<2.08$ \\
\hline & Minimum corrected weight of impurity in sample $(\mu \mathrm{g})$ : & 0.00 & 0.00 & 0.00 \\
\hline & Maximum corrected weight of impurity in sample $(\mu \mathrm{g})$ : & 1.07 & 1.01 & 2.08 \\
\hline \multirow{5}{*}{$\mathrm{Ca}$} & Measured concentration of impurity in sample $(\mu \mathrm{g} / \mathrm{ml})$ : & $<1.00 \mathrm{E}-01$ & $<1.00 \mathrm{E}-01$ & $\mathrm{Ca}$ \\
\hline & Uncorrected weight of impurity in sample $(\mu \mathrm{g})$ : & $<13.40$ & $<12.60$ & $<26.00$ \\
\hline & Weight of impurity in blank $(\mu \mathrm{g})$ : & $<15.90$ & $<12.30$ & \\
\hline & Minimum corrected welght of impurity in sample $(\mu \mathrm{g})$ : & 0.00 & 0.00 & 0.00 \\
\hline & Maximum corrected weight of impurity in sample $(\mu \mathrm{g})$ : & 13.40 & 12.60 & 26.00 \\
\hline \multirow{5}{*}{ Al } & Measured concentration of impurity in sample $(\mu \mathrm{g} / \mathrm{ml})$ : & $4.56 \mathrm{E}-01$ & $1.28 \mathrm{E}-01$ & Al \\
\hline & Uncorrected weight of impurity in sample $(\mu \mathrm{g})$ : & 61.10 & 16.13 & 77.23 \\
\hline & Weight of impurity in blank $(\mu \mathrm{g})$ : & 5.99 & 3.48 & \\
\hline & Minimum corrected weight of impurity in sample $(\mu \mathrm{g})$ : & 55.11 & 12.65 & 67.76 \\
\hline & Maximum corrected weight of impurity in sample $(\mu \mathrm{g})$ : & 55.11 & 12.65 & 67.76 \\
\hline \multirow{5}{*}{$\mathbf{T i}$} & Measured concentration of impurity in sample $(\mu \mathrm{g} / \mathrm{ml})$ : & $2.31 \mathrm{E}-02$ & $1.97 \mathrm{E}-02$ & $\mathrm{Ti}$ \\
\hline & Uncorrected weight of impurity in sample $(\mu \mathrm{g})$ : & 3.10 & 2.48 & 5.58 \\
\hline & $\begin{array}{l}\text { Weight of impurity in blank }(\mu \mathrm{g}) \text { : } \\
\text {. }\end{array}$ & $<1.27$ & $<0.98$ & \\
\hline & Minimum corrected weight of impurity in sample $(\mathrm{\mu g})$ : & 1.82 & 1.50 & 3.32 \\
\hline & Maximum corrected weight of impurity in sample ( $(\mathrm{gg})$ : & 3.10 & 2.48 & 5.58 \\
\hline \multirow{5}{*}{$\mathbf{v}$} & Measured concentration of impurity in sample $(\mu \mathrm{g} / \mathrm{ml})$ : & $2.47 \mathrm{E}-01$ & $5.51 \mathrm{E}-02$ & $\mathrm{v}$ \\
\hline & Uncorrected weight of impurity in sample $(\mu g)$ : & 33.10 & 6.94 & 40.04 \\
\hline & Weight of impurity in blank $(\mu g)$ : & $<0.32$ & $<0.25$ & \\
\hline & Minimum corrected weight of impurity in sample $(\mu \mathrm{g})$ : & 32.78 & 6.70 & 39.48 \\
\hline & Maximum corrected weight of impurity in sample $(\mu q)$ : & 33.10 & 6.94 & 40.04 \\
\hline
\end{tabular}

Comments

Data checked against RMAL 2134 official results of analyses by FCM on 10/14/2009 
Data Report Form DRF-26A: Measurement of U Contamination or Impurities by Deconsolidation Leach

\begin{tabular}{|c|c|}
\hline Procedure: & AGR-CHAR-DAM-26 Rev, 1 \\
\hline Operator: & Fred Montgomery \\
\hline Compact lot ID: & LEU09-OP2-Z \\
\hline Compact lot description: & AGR-2 UCO Variant, from G73J-14-93073A \\
\hline Compact ID numbers: & $067,081,019,008,032$ \\
\hline DRF filename: & IImc-agr|AGRILeachBurnLeach|LEU09-OP2-Z-DRF26R1_01.xls \\
\hline
\end{tabular}

\begin{tabular}{|c|c|c|c|c|}
\hline \multirow{2}{*}{\multicolumn{2}{|c|}{$\begin{array}{r}\text { Mean average weight uranium per particle }(\mathrm{g}) \\
\text { Uncertainty in mean averaqe weight uranium per particle }(\mathrm{q}): \\
\end{array}$}} & \multicolumn{3}{|c|}{$3.96 \mathrm{E}-04$} \\
\hline & & \multicolumn{3}{|c|}{$8.10 \mathrm{E}-07$} \\
\hline & & First Leach & Second Leach & Total \\
\hline & Deconsolidation-leach solution ID: & L09080303 & L09080503 & \\
\hline & Number of compacts: & & & \\
\hline & Total volume of leach solution $(\mathrm{ml})$ : & 135.0 & 121.0 & \\
\hline & Radiochemical laboratory analysis number: & $2134-003$ & $2134-008$ & \\
\hline & Measured uranium concentration $(\mu \mathrm{g} / \mathrm{ml})$ : & $7.15 E-02$ & $8.66 \mathrm{E}-03$ & \\
\hline & Uncertainty in uranium concentration $(\mu \mathrm{g} / \mathrm{ml})$ : & $7.15 E-03$ & $8.66 \mathrm{E}-04$ & \\
\hline & Weight uranium leached $(g):$ & $9.65 E-06$ & $1.05 E-06$ & 1.07E-05 \\
\hline & Uncertainty in weight uranium leached $(g)$ : & $9.66 \mathrm{E}-07$ & $1.05 \mathrm{E}-07$ & $9.72 \mathrm{E}-07$ \\
\hline & Effective number of exposed kernels: & 0.0 & 0.0 & 0.0 \\
\hline & Uncertainty in effective number of exposed kernels: & 0.0 & 0.0 & 0.0 \\
\hline & & & & \\
\hline \multirow{5}{*}{$\mathbf{F e}$} & Measured concentration of impurity in sample $(\mu \mathrm{g} / \mathrm{ml})$ : & $<4.12 \mathrm{E}-02$ & $<4.12 \mathrm{E}-02$ & $\mathrm{Fe}$ \\
\hline & Uncorrected weight of impurity in sample $(\mu \mathrm{g})$ : & $<5.56$ & $<4.99$ & $<10.55$ \\
\hline & Weight of impurity in blank $(\mu \mathrm{g})$ : & $<6.55$ & $<5.07$ & \\
\hline & Minimum corrected weight of impurity in sample $(\mu \mathrm{g})$ : & 0.00 & 0.00 & 0.00 \\
\hline & Maximum corrected weight of impurity in sample $(\mu \mathrm{g})$ : & 5.56 & 4.99 & 10.55 \\
\hline \multirow{5}{*}{$\mathrm{Cr}$} & Measured concentration of impurity in sample $(\mu \mathrm{g} / \mathrm{ml})$ : & $4.61 \mathrm{E}-03$ & $4.31 \mathrm{E}-03$ & $\mathrm{Cr}$ \\
\hline & Uncorrected weight of impurity in sample $(\mu \mathrm{g})$ : & 0.62 & 0.52 & 1.14 \\
\hline & Weight of impurity in blank $(\mu \mathrm{g})$ : & $<0.32$ & $<0.25$ & \\
\hline & Minimum corrected weight of impurity in sample $(\mu \mathrm{g})$ : & 0.30 & 0.28 & 0.58 \\
\hline & Maximum corrected weight of impurity in sample $(\mu \mathrm{g})$ : & 0.62 & 0.52 & 1.14 \\
\hline \multirow{5}{*}{ Mn } & Measured concentration of impurity in sample $(\mu \mathrm{g} / \mathrm{ml})$ : & $<1.91 \mathrm{E}-03$ & $<1.91 \mathrm{E}-03$ & Mn \\
\hline & Uncorrected weight of impurity in sample $(\mu \mathrm{g})$ : & $<0.26$ & $<0.23$ & $<0.49$ \\
\hline & Weight of impurity in blank $(\mu \mathrm{g})$ : & $<0.30$ & $<0.23$ & \\
\hline & Minimum corrected weight of impurity in sample $(\mu \mathrm{g})$ : & 0.00 & 0.00 & 0.00 \\
\hline & Maximum corrected weight of impurity in sample $(\mu \mathrm{g})$ : & 0.26 & 0.23 & 0.49 \\
\hline \multirow{5}{*}{ Co } & Measured concentration of impurity in sample $(\mu \mathrm{g} / \mathrm{ml})$ : & $<1.62 \mathrm{E}-03$ & $<1.62 \mathrm{E}-03$ & Co \\
\hline & Uncorrected weight of impurity in sample $(\mu \mathrm{g})$ : & $<0.22$ & $<0.20$ & $<0.41$ \\
\hline & Weight of impurity in blank $(\mu \mathrm{g})$ : & $<0.26$ & $<0.20$ & \\
\hline & Minimum corrected weight of impurity in sample $(\mu \mathrm{g})$ : & 0.00 & 0.00 & 0.00 \\
\hline & Maximum corrected weight of impurity in sample $(\mu \mathrm{g})$ : & 0.22 & 0.20 & 0.41 \\
\hline \multirow{5}{*}{ Ni } & Measured concentration of impurity in sample $(\mu \mathrm{g} / \mathrm{ml})$ : & $1.14 \mathrm{E}-02$ & $<8.00 \mathrm{E}-03$ & $\mathrm{Ni}$ \\
\hline & Uncorrected weight of impurity in sample $(\mu g)$ : & 1.54 & $<0.97$ & $<2.51$ \\
\hline & Weight of impurity in blank $(\mu \mathrm{g})$ : & $<1.27$ & $<0.98$ & \\
\hline & Minimum corrected weight of impurity in sample $(\mu \mathrm{g})$ : & 0.27 & 0.00 & 0.27 \\
\hline & Maximum corrected weight of impurity in sample $(\mu \mathrm{g})$ : & 1.54 & 0.97 & 2.51 \\
\hline \multirow{5}{*}{$\mathbf{C a}$} & Measured concentration of impurity in sample $(\mu \mathrm{g} / \mathrm{ml})$ : & 1.93E-01 & $1.03 E-01$ & $\mathrm{Ca}$ \\
\hline & Uncorrected weight of impurity in sample $(\mu \mathrm{g})$ : & 26.06 & 12.46 & 38.52 \\
\hline & Weight of impurity in blank $(\mu \mathrm{g})$ : & $<15.90$ & $<12.30$ & \\
\hline & Minimum corrected weight of impurity in sample $(\mu \mathrm{g})$ : & 10.16 & 0.16 & 10.32 \\
\hline & Maximum corrected weight of impurity in sample $(\mu \mathrm{g})$ : & 26.06 & 12.46 & 38.52 \\
\hline \multirow{5}{*}{ Al } & Measured concentration of impurity in sample $(\mu \mathrm{g} / \mathrm{ml})$ : & 3.95E-01 & $8.21 E-02$ & Al \\
\hline & Uncorrected weight of impurity in sample $(\mu \mathrm{g})$ : & 53.33 & 9.93 & 63.26 \\
\hline & Weight of impurity in blank $(\mu \mathrm{g})$ : & 5.99 & 3.48 & \\
\hline & Minimum corrected weight of impurity in sample $(\mu \mathrm{g})$ : & 47.33 & 6.45 & 53.78 \\
\hline & Maximum corrected weight of impurity in sample $(\mu \mathrm{g})$ : & 47.33 & 6.45 & 53.78 \\
\hline \multirow{5}{*}{$\mathrm{Ti}$} & Measured concentration of impurity in sample $(\mu \mathrm{g} / \mathrm{ml})$ : & $2.14 \mathrm{E}-02$ & 1.91E-02 & $\mathrm{Ti}$ \\
\hline & Uncorrected weight of impurity in sample $(\mu \mathrm{g})$ : & 2.89 & 2.31 & 5.20 \\
\hline & Weight of impurity in blank $(\mu \mathrm{g})$ : & $<1.27$ & $<0.98$ & \\
\hline & Minimum corrected weight of impurity in sample $(\mu \mathrm{g})$ : & 1.62 & 1.33 & 2.94 \\
\hline & Maximum corrected weight of impurity in sample $(\mu \mathrm{g})$ : & 2.89 & 2.31 & 5.20 \\
\hline \multirow{5}{*}{$\mathbf{v}$} & Measured concentration of impurity in sample $(\mu \mathrm{g} / \mathrm{ml})$ : & $2.51 E-01$ & $5.57 \mathrm{E}-02$ & $\mathbf{v}$ \\
\hline & Uncorrected weight of impurity in sample $(\mu \mathrm{g})$ : & 33.89 & 6.74 & 40.62 \\
\hline & Welght of impurity in blank $(\mu \mathrm{g})$ : & $<0.32$ & $<0.25$ & \\
\hline & Minimum corrected weight of impurity in sample $(\mu \mathrm{g})$ : & 33.57 & 6.49 & 40.06 \\
\hline & Maximum corrected weight of impurity in sample $(\mu \mathrm{g})$ : & 33.89 & 6.74 & 40.62 \\
\hline
\end{tabular}

Comments

Data checked against RMAL 2134 official results of analyses by FCM on 10/14/2009

Fied C. Montgomely

$1-2.7 .2010$ 
Data Report Form DRF-26A: Measurement of U Contamination or Impurities by Deconsolidation Leach

\begin{tabular}{|c|c|}
\hline Procedure: & AGR-CHAR-DAM-26 Rev. 1 \\
\hline $\begin{array}{r}\text { Operator: } \\
\text { Compact lot ID: }\end{array}$ & Fred Montgomery \\
\hline $\begin{array}{r:}\text { Compact lot ID: } \\
\text { Compact lot description: }\end{array}$ & LEU09-OP2-Z \\
\hline$\frac{\text { Compact lot description: }}{\text { Compact ID numbers: }}$ & AGR-2 UCO Variant, from G73J-14-93073A \\
\hline Compact ID numbers: & 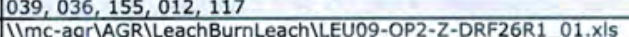 \\
\hline
\end{tabular}

\begin{tabular}{|c|c|c|c|c|}
\hline \multirow{2}{*}{\multicolumn{2}{|c|}{\begin{tabular}{|c|} 
Mean average weight uranium per particle $(\mathrm{g})$ : \\
Uncertainty in mean average weight uranium per particle $(q)$ :
\end{tabular}}} & \multicolumn{3}{|c|}{$\begin{array}{l}3.96 \mathrm{E}-04 \\
8.10 \mathrm{E}-07\end{array}$} \\
\hline & & First Leach & Second Leach & Total \\
\hline & Deconsolidation-leach solution ID: & L09080304 & L09080504 & \\
\hline & Number of compacts: & & & \\
\hline & Total volume of leach solution (ml): & 139.0 & 126.0 & \\
\hline & Radiochemical laboratory analysis number: & $2134-004$ & $2134-009$ & \\
\hline & Measured uranium concentration $(\mathrm{\mu g} / \mathrm{ml})$ : & $7.19 \mathrm{E}-02$ & $1.01 \mathrm{E}-02$ & \\
\hline & Uncertainty in uranium concentration $(\mu \mathrm{g} / \mathrm{ml})$ : & $7.19 \mathrm{E}-03$ & $1.01 \mathrm{E}-03$ & \\
\hline & Weight uranium leached (9): & $9.99 \mathrm{E}-06$ & $1.27 E-06$ & $1.13 \mathrm{E}-05$ \\
\hline & Uncertainty in weight uranium leached $(\mathrm{g})$ : & $1.00 \mathrm{E}-06$ & $1.27 E-07$ & $1.01 \mathrm{E}-06$ \\
\hline & Effective number of exposed kernels: & 0.0 & 0.0 & 0.0 \\
\hline & Uncertainty in effective number of exposed kernels: & 0.0 & 0.0 & 0.0 \\
\hline \multirow{5}{*}{$\mathbf{F e}$} & Measured concentration of impurity in sample $(\mu \mathrm{g} / \mathrm{ml})$ : & $<4.12 \mathrm{E}-02$ & $<4.12 \mathrm{E}-02$ & $\mathbf{F e}$ \\
\hline & Uncorrected weight of impurity in sample $(\mu g)$ : & $<5.73$ & $<5.19$ & $<10.92$ \\
\hline & Weight of impurity in blank $(\mu g)$ : & $<6.55$ & $<5.07$ & \\
\hline & Minimum corrected weight of impurity in sample $(\mu g)$ : & 0.00 & 0.00 & 0.00 \\
\hline & Maximum corrected weight of impurity in sample $(\mu g)$ : & 5.73 & 5.19 & 10.92 \\
\hline \multirow{5}{*}{ Cr } & Measured concentration of impurity in sample $(\mu \mathrm{g} / \mathrm{ml})$ : & $1.01 \mathrm{E}-02$ & $7.10 \mathrm{E}-03$ & $\mathrm{Cr}$ \\
\hline & Uncorrected weight of impurity in sample $(\mu g)$ : & 1.40 & 0.89 & 2.30 \\
\hline & Weight of impurity in blank $(\mu \mathrm{g})$ : & $<0.32$ & $<0.25$ & \\
\hline & Minimum corrected weight of impurity in sample $(\mu g)$ : & 1.09 & 0.65 & 1.73 \\
\hline & Maximum corrected weight of impurity in sample $(\mu g)$ : & 1.40 & 0.89 & 2.30 \\
\hline \multirow{5}{*}{$\mathrm{Mn}$} & Measured concentration of impurity in sample $(\mathrm{\mu g} / \mathrm{ml})$ : & $<1.91 \mathrm{E}-03$ & $<1.91 \mathrm{E}-03$ & $\mathrm{Mn}$ \\
\hline & Uncorrected weight of impurity in sample $(\mu g)$ : & $<0.27$ & $<0.24$ & $<0.51$ \\
\hline & Weight of impurity in blank $(\mu \mathrm{g})$ : & $<0.30$ & $<0.23$ & \\
\hline & Minimum corrected weight of impurity in sample $(\mu g)$ : & 0.00 & 0.00 & 0.00 \\
\hline & Maximum corrected weight of impurity in sample $(\mu g)$ : & 0.27 & 0.24 & 0.51 \\
\hline \multirow{5}{*}{ co } & Measured concentration of impurity in sample $(\mu \mathrm{g} / \mathrm{ml})$ : & $<1.62 \mathrm{E}-03$ & $<1.62 \mathrm{E}-03$ & Co \\
\hline & Uncorrected weight of impurity in sample $(\mu g)$ : & $<0.23$ & $<0.20$ & $<0.43$ \\
\hline & Weight of impurity in blank $(\mu \mathrm{g})$ : & $<0.26$ & $<0.20$ & \\
\hline & Minimum corrected weight of impurity in sample $(\mu g)$ : & 0.00 & 0.00 & 0.00 \\
\hline & Maximum corrected weight of impurity in sample $(\mu g)$ : & 0.23 & 0.20 & 0.43 \\
\hline \multirow{5}{*}{$\mathbf{N i}$} & Measured concentration of impurity in sample $(\mu \mathrm{g} / \mathrm{ml})$ : & $<8.00 \mathrm{E}-03$ & $<8.00 \mathrm{E}-03$ & $\mathrm{Ni}$ \\
\hline & Uncorrected weight of impurity in sample $(\mu \mathrm{g})$ : & $<1.11$ & $<1.01$ & $<2.12$ \\
\hline & Weight of impurity in blank $(\mu g)$ : & $<1.27$ & $<0.98$ & \\
\hline & Minimum corrected weight of impurity in sample $(\mu g)$ : & 0.00 & 0.00 & 0.00 \\
\hline & Maximum corrected weight of impurity in sample $(\mu \mathrm{g})$ : & 1.11 & 1.01 & 2.12 \\
\hline \multirow{5}{*}{$\mathrm{Ca}$} & Measured concentration of impurity in sample $(\mu \mathrm{g} / \mathrm{ml})$ : & $4.74 \mathrm{E}-01$ & $<1.00 \mathrm{E}-01$ & $\mathrm{Ca}$ \\
\hline & Uncorrected weight of impurity in sample $(\mu g)$ : & 65.89 & $<12.60$ & $<78.49$ \\
\hline & Weight of impurity in blank $(\mu \mathrm{g})$ : & $<15.90$ & $<12.30$ & \\
\hline & Minimum corrected weight of impurity in sample $(\mu \mathrm{g})$ : & 49.99 & 0.00 & 49.99 \\
\hline & Maximum corrected weight of impurity in sample $(\mu g)$ : & 65.89 & 12.60 & 78.49 \\
\hline \multirow{5}{*}{ Al } & Measured concentration of impurity in sample $(\mu \mathrm{g} / \mathrm{ml})$ : & $4.34 \mathrm{E}-01$ & $1.06 \mathrm{E}-01$ & Al \\
\hline & Uncorrected weight of impurity in sample $(\mu g)$ : & 60.33 & 13.36 & 73.68 \\
\hline & Weight of impurity in blank $(\mu g)$ : & 5.99 & 3.48 & \\
\hline & Minimum corrected weight of impurity in sample $(\mu g)$ : & 54.33 & 9.88 & 64.21 \\
\hline & Maximum corrected weight of impurity in sample $(\mu \mathrm{g})$ : & 54.33 & 9.88 & 64.21 \\
\hline \multirow{5}{*}{$\mathbf{T i}$} & Measured concentration of Impurity in sample $(\mu \mathrm{g} / \mathrm{ml})$ : & $4.88 \mathrm{E}-02$ & $3.50 \mathrm{E}-02$ & $\mathrm{Ti}$ \\
\hline & Uncorrected weight of impurity in sample $(\mu \mathrm{g})$ : & 6.78 & 4.41 & 11.19 \\
\hline & Weight of impurity in blank $(\mu \mathrm{g})$ : & $<1.27$ & $<0.98$ & \\
\hline & Minimum corrected weight of impurity in sample $(\mu \mathrm{g})$ : & 5.51 & 3.43 & 8.94 \\
\hline & Maximum corrected weight of impurity in sample $(\mu g)$ : & 6.78 & 4.41 & 11.19 \\
\hline \multirow{5}{*}{$\mathbf{v}$} & Measured concentration of impurity in sample $(\mu \mathrm{g} / \mathrm{ml})$ : & $2.48 \mathrm{E}-01$ & $4.64 \mathrm{E}-02$ & $\mathrm{v}$ \\
\hline & Uncorrected weight of impurity in sample $(\mu \mathrm{g})$ : & 34.47 & 5.85 & 40.32 \\
\hline & Weight of impurity in blank $(\mu \mathrm{g})$ : & $<0.32$ & $<0.25$ & \\
\hline & Minimum corrected weight of impurity in sample $(\mu \mathrm{g})$ : & 34.15 & 5.60 & 39.75 \\
\hline & Maximum corrected weight of impurity in sample $(\mu g)$ : & 34.47 & 5.85 & 40.32 \\
\hline \multicolumn{5}{|c|}{ Commen } \\
\hline \multicolumn{5}{|c|}{ Data checked against RMAL 2134 official results of analyses by FCM on 10/14/2009 } \\
\hline
\end{tabular}

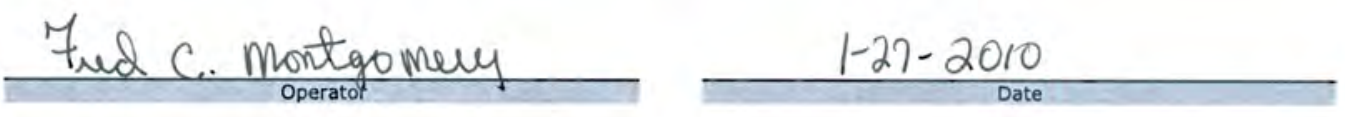


Data Report Form DRF-26A: Measurement of $U$ Contamination or Impurities by Deconsolidation Leach

\begin{tabular}{|c|c|}
\hline Procedure: & AGR-CHAR-DAM-26 Rev. 1 \\
\hline $\begin{array}{r}\text { Operator: } \\
\text { Compact lot ID: }\end{array}$ & Fred Montgomery \\
\hline Compact lot ID: & LEU09-OP2-Z \\
\hline Compact lot description: & AGR-2 UCO Variant, from G73J-14-93073A \\
\hline Compact ID numbers: & $031,149,093,147,072$ \\
\hline DRF filename: & Imc-agr|AGRILeachBurnt \\
\hline
\end{tabular}

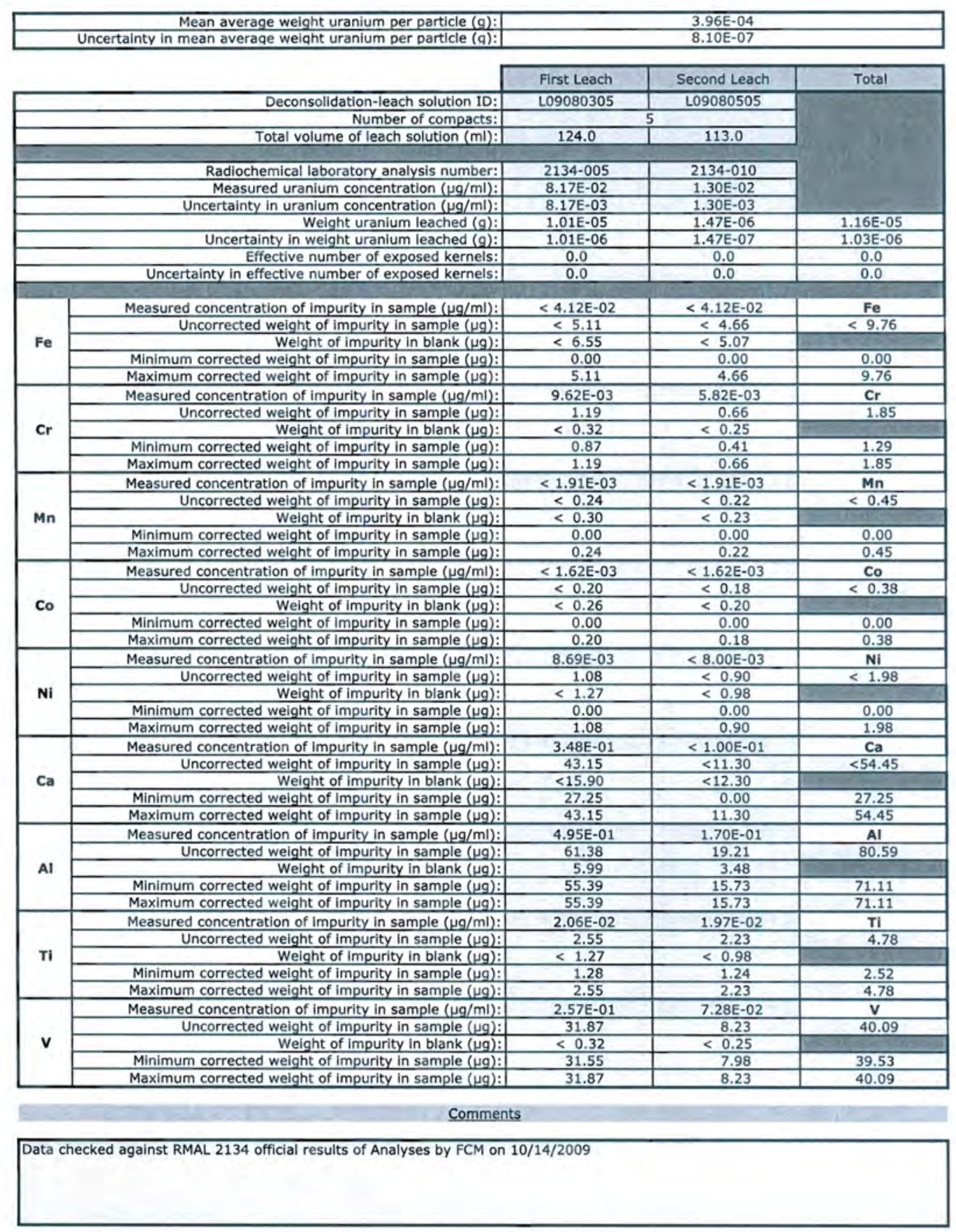

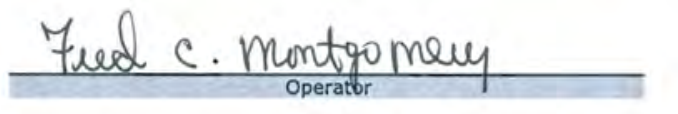

$1-27-2010$ 
Data Report Form DRF-26A: Measurement of U Contamination or Impurities by Deconsolidation Leach

\begin{tabular}{|r|l|}
\hline Procedure: & AGR-CHAR-DAM-26 Rev. 1 \\
\hline Operator: & Fred Montgomery \\
\hline Compact lot ID: & LEU09-OP2-Z \\
\hline Compact lot description: & AGR-2 UCO Variant, from G73J-14-93073A \\
\hline Compact ID numbers: & Deconsolidation Leach Blank \\
\hline DRF filename: & IImc-agr\AGRILeachBurnLeachILEU09-OP2-Z-DRF26R1_01.xIs \\
\hline
\end{tabular}

\begin{tabular}{|c|c|c|c|c|}
\hline \multirow{2}{*}{\multicolumn{2}{|c|}{\begin{tabular}{r|} 
Mean average weight uranium per particle $(\mathrm{g})$ : \\
Uncertainty in mean average weight uranium per particle $(\mathrm{g}):$
\end{tabular}}} & \multicolumn{3}{|c|}{$3.96 \mathrm{E}-04$} \\
\hline & & \multicolumn{3}{|c|}{$8.10 \mathrm{E}-07$} \\
\hline & & First Leach & Second Leach & Total \\
\hline & Deconsolidation-leach solution ID: & L09080302 & L09080502 & \\
\hline & Number of compacts: & \multicolumn{2}{|c|}{ None } & \\
\hline & Total volume of leach solution $(\mathrm{ml})$ : & 159.0 & 123.0 & \\
\hline 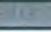 & & & & \\
\hline & Radiochemical laborator & -002 & 2134-007 & \\
\hline & Measured uranium concentration $(\mu \mathrm{g} / \mathrm{ml})$ : & $<2.00 \mathrm{E}-04$ & $2.24 \mathrm{E}-04$ & \\
\hline & Uncertainty in uranium concentration $(\mu \mathrm{g} / \mathrm{ml})$ : & & $2.24 \mathrm{E}-05$ & \\
\hline & Weight uranium leached $(\mathrm{g})$ : & $<3.18 \mathrm{E}-08$ & $2.76 \mathrm{E}-08$ & $<5.94 E-08$ \\
\hline & Uncertainty in weight uranium leached $(g)$ : & & $2.76 \mathrm{E}-09$ & \\
\hline & Effective number of exposed kernels: & 0.0 & 0.0 & 0.0 \\
\hline & Uncertainty in effective number of exposed kernels: & & 0.0 & \\
\hline & & & & \\
\hline \multirow{2}{*}{$\mathbf{F e}$} & Measured concentration $(\mu \mathrm{g} / \mathrm{ml})$ : & $<4.12 \mathrm{E}-02$ & $<4.12 \mathrm{E}-02$ & $\mathrm{Fe}$ \\
\hline & Total weight of leached impurity $(\mu \mathrm{g})$ : & $<6.55$ & $<5.07$ & $\leq 11.62$ \\
\hline \multirow{2}{*}{$\mathrm{Cr}$} & Measured concentration $(\mu \mathrm{g} / \mathrm{ml})$ : & $<2.00 \mathrm{E}-03$ & $<2.00 \mathrm{E}-03$ & $\mathrm{Cr}$ \\
\hline & Total weight of leached impurity $(\mu \mathrm{g})$ : & $<0.32$ & $<0.25$ & $<0.56$ \\
\hline \multirow{2}{*}{ Mn } & Measured concentration $(\mu \mathrm{g} / \mathrm{ml})$ : & $<1.91 \mathrm{E}-03$ & $<1.91 \mathrm{E}-03$ & Mn \\
\hline & Total weight of leached impurity $(\mu \mathrm{g})$ : & $<0.30$ & $<0.23$ & $<0.54$ \\
\hline \multirow{2}{*}{ Co } & Measured concentration $(\mu \mathrm{g} / \mathrm{ml})$ : & $<1.62 \mathrm{E}-03$ & $<1.62 \mathrm{E}-03$ & Co \\
\hline & Total weight of leached impurity $(\mu \mathrm{g})$ : & $<0.26$ & $<0.20$ & $<0.46$ \\
\hline \multirow{2}{*}{$\mathbf{N i}$} & Measured concentration $(\mu \mathrm{g} / \mathrm{ml})$ : & $<8.00 \mathrm{E}-03$ & $<8.00$ E-03 & $\mathrm{Ni}$ \\
\hline & Total weight of leached impurity $(\mu \mathrm{g})$ : & $<1.27$ & $<0.98$ & $<2.26$ \\
\hline \multirow{2}{*}{$\mathrm{Ca}$} & Measured concentration $(\mu \mathrm{g} / \mathrm{ml})$ : & $<1.00 \mathrm{E}-01$ & $<1.00 \mathrm{E}-01$ & $\mathrm{Ca}$ \\
\hline & Total weight of leached impurity $(\mu \mathrm{g})$ : & $<15.90$ & $<12.30$ & $<28.20$ \\
\hline \multirow{2}{*}{ Al } & Measured concentration $(\mu \mathrm{g} / \mathrm{ml})$ : & $3.77 \mathrm{E}-02$ & $2.83 E-02$ & $\overline{A l}$ \\
\hline & Total weight of leached impurity $(\mu \mathrm{g})$ : & 5.99 & 3.48 & 9.48 \\
\hline \multirow{2}{*}{$\mathbf{T i}$} & Measured concentration $(\mu \mathrm{g} / \mathrm{ml})$ : & $<8.00 \mathrm{E}-03$ & $<8.00$ E- 03 & $\mathrm{Ti}$ \\
\hline & Total weight of leached impurity $(\mu \mathrm{g})$ : & $<1.27$ & $<0.98$ & $<2.26$ \\
\hline \multirow{2}{*}{$\mathbf{v}$} & Measured concentration $(\mu \mathrm{g} / \mathrm{ml})$ : & $<2.00 \mathrm{E}-03$ & $<2.00 \mathrm{E}-03$ & v \\
\hline & Total weight of leached impurity $(\mu \mathrm{g})$ : & $<0.32$ & $<0.25$ & $<0.56$ \\
\hline
\end{tabular}

\section{Comments}

Data checked against RMAL 2134 official results of analyses by FCM on 10/14/2009

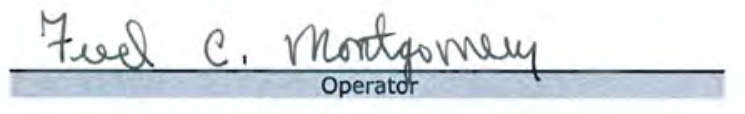

$1-27-2010$

Date 
Data Report Form DRF-26B: Measurement of SIC Burn-Leach Defects or Impurities by Burn-Leach

\begin{tabular}{|c|c|}
\hline Procedure: & AGR-CHAR-DAM-26 Rev. 1 \\
\hline Operator: & Fred Montgomery \\
\hline Compact lot ID: & LEU09-OPZ-Z \\
\hline Compact lot description: & AGR-2 UCO Variant, from G73]-14-93073A \\
\hline Compact ID numbers: & $011,152,001,068,046$ \\
\hline DRF filename: & IImc-agr|AGRILeachBurnLeach (LEU09-OP2-Z-DRF26R1_01.xls \\
\hline
\end{tabular}

\begin{tabular}{|c|c|c|c|c|}
\hline \multicolumn{2}{|r|}{$\begin{array}{l}\text { Mean average weight uranium per particle }(g): \\
\text { Uncertainty in mean averaqe weight uranium per particle }(q):\end{array}$} & \multicolumn{3}{|c|}{$\begin{array}{l}3.96 \mathrm{E}-04 \\
8.10 \mathrm{E}-07\end{array}$} \\
\hline & & First Leach & Second Leach & Total \\
\hline & Burn-leach solution ID: & B09081401 & B09081701 & \\
\hline & Number of compacts: & & & \\
\hline & Total volume of leach solution $(\mathrm{ml})$ : & 51.0 & 52.0 & \\
\hline & Radiochemical laboratory analysis number: & $2157-016$ & $2157-021$ & \\
\hline & Measured uranium concentration $(\mathrm{\mu g} / \mathrm{ml})$ : & $3.11 \mathrm{E}-01$ & $2.89 \mathrm{E}-03$ & \\
\hline & Uncertainty in uranium concentration $(\mu \mathrm{g} / \mathrm{ml})$ : & $3.11 \mathrm{E}-02$ & $2.89 \mathrm{E}-04$ & \\
\hline & Weight uranium leached (g): & $1.59 \mathrm{E}-05$ & $1.50 \mathrm{E}-07$ & $1.60 \mathrm{E}-05$ \\
\hline & Uncertainty in weight uranium leached $(\mathrm{g})$ : & $1.60 \mathrm{E}-06$ & $1.51 \mathrm{E}-08$ & $1.60 \mathrm{E}-06$ \\
\hline & Number of leached kernels: & 0.0 & 0.0 & 0.0 \\
\hline & Uncertainty in number of leached kernels: & 0.0 & 0.0 & 0.0 \\
\hline \multirow{5}{*}{$\mathbf{F e}$} & Measured concentration of impurity in sample $(\mu \mathrm{g} / \mathrm{ml})$ : & $7.89 E-02$ & $<4.12 \mathrm{E}-02$ & $\mathbf{F e}$ \\
\hline & Uncorrected weight of impurity in sample $(\mu \mathrm{g})$ : & 4.02 & $<2.14$ & $<6.17$ \\
\hline & Weight of impurity in blank $(\mu g)$ : & $<2.15$ & $<2.10$ & \\
\hline & Minimum corrected weight of impurity in sample $(\mu q)$ : & 1.87 & 0.00 & 1.87 \\
\hline & Maximum corrected weight of impurity in sample $(\mu g)$ : & 4.02 & 2.14 & 6.17 \\
\hline \multirow{5}{*}{$\mathrm{Cr}$} & Measured concentration of impurity in sample $(\mathrm{\mu g} / \mathrm{ml})$ : & $2.26 \mathrm{E}-02$ & $<2.00 \mathrm{E}-03$ & $\mathrm{Cr}$ \\
\hline & Uncorrected weight of impurity in sample $(\mu \mathrm{g})$ : & 1.15 & $<0.10$ & $<1.26$ \\
\hline & Weight of impurity in blank $(\mu g)$ : & $<0.10$ & $<0.10$ & \\
\hline & Minimum corrected weight of impurity in sample $(\mu g)$ : & 1.05 & 0.00 & 1.05 \\
\hline & Maximum corrected weight of impurity in sample $(\mu g)$ : & 1.15 & 0.10 & 1.26 \\
\hline \multirow{5}{*}{ Mn } & Measured concentration of impurity in sample $(\mu \mathrm{g} / \mathrm{ml})$ : & $<1.91 \mathrm{E}-03$ & $<1.91 \mathrm{E}-03$ & $\mathrm{Mn}$ \\
\hline & Uncorrected weight of impurity in sample $(\mu g)$ : & $<0.10$ & $<0.10$ & $<0.20$ \\
\hline & Weight of impurity in blank $(\mu g)$ : & $<0.10$ & $<0.10$ & \\
\hline & Minimum corrected weight of impurity in sample $(\mu \mathrm{g})$ : & 0.00 & 0.00 & 0.00 \\
\hline & Maximum corrected weight of impurity in sample $(\mu g)$ : & 0.10 & 0.10 & 0.20 \\
\hline \multirow{5}{*}{ co } & Measured concentration of impurity in sample $(\mathrm{\mu g} / \mathrm{ml})$ : & $<1.62 \mathrm{E}-03$ & $<1.62 \mathrm{E}-03$ & Co \\
\hline & Uncorrected weight of Impurity in sample $(\mu g)$ : & $<0.08$ & $<0.08$ & $<0.17$ \\
\hline & Weight of impurity in blank $(\mu g)$ : & $<0.08$ & $<0.08$ & \\
\hline & Minimum corrected weight of impurity in sample $(\mu g)$ : & 0.00 & 0.00 & 0.00 \\
\hline & Maximum corrected weight of impurity in sample $(\mu g)$ : & 0.08 & 0.08 & 0.17 \\
\hline \multirow{5}{*}{ Ni } & Measured concentration of impurity in sample $(\mathrm{\mu g} / \mathrm{ml})$ : & $3.94 \mathrm{E}-02$ & $<8.00 \mathrm{E}-03$ & $\mathrm{Ni}$ \\
\hline & Uncorrected weight of impurity in sample $(\mu \mathrm{g})$ : & 2.01 & $<0.42$ & $<2.43$ \\
\hline & Weight of impurity in blank $(\mu \mathrm{g})$ : & $<0.42$ & $<0.41$ & \\
\hline & Minimum corrected weight of impurity in sample $(\mu \mathrm{g})$ : & 1.59 & 0.00 & 1.59 \\
\hline & Maximum corrected weight of impurity in sample $(\mu g)$ : & 2.01 & 0.42 & 2.43 \\
\hline \multirow{5}{*}{$\mathrm{Ca}$} & Measured concentration of impurity in sample $(\mu \mathrm{g} / \mathrm{ml})$ : & $2.52 \mathrm{E}+00$ & $1.68 \mathrm{E}+00$ & $\mathrm{Ca}$ \\
\hline & Uncorrected weight of impurity in sample $(\mu g)$ : & 128.52 & 87.36 & 215.88 \\
\hline & Weight of impurity in blank $(\mu g)$ : & 9.92 & 97.41 & \\
\hline & Minimum corrected weight of impurity in sample $(\mu \mathrm{g})$ : & 118.60 & 0.00 & 118.60 \\
\hline & Maximum corrected weight of impurity in sample $(\mu g)$ : & 118.60 & 0.00 & 118.60 \\
\hline \multirow{5}{*}{ Al } & Measured concentration of impurity in sample $(\mu \mathrm{g} / \mathrm{ml})$ : & $1.58 \mathrm{E}+00$ & $4.90 \mathrm{E}-02$ & Al \\
\hline & Uncorrected weight of impurity in sample $(\mu g)$ : & 80.58 & 2.55 & 83.13 \\
\hline & Weight of impurity in blank $(\mu g)$ : & 3.05 & 1.28 & \\
\hline & Minimum corrected weight of impurity in sample $(\mu g)$ : & 77.53 & 1.27 & 78.80 \\
\hline & Maximum corrected weight of impurity in sample $(\mu g)$ : & 77.53 & 1.27 & 78.80 \\
\hline \multirow{5}{*}{ Ti } & Measured concentration of impurity in sample $(\mu \mathrm{g} / \mathrm{ml})$ : & $1.44 \mathrm{E}-01$ & $<8.00 \mathrm{E}-03$ & $\mathrm{Ti}$ \\
\hline & Uncorrected weight of impurity in sample $(\mu \mathrm{g})$ : & 7.34 & $<0.42$ & $<7.76$ \\
\hline & Weight of impurity in blank $(\mu \mathrm{g})$ : & $<0.42$ & $<0.41$ & \\
\hline & Minimum corrected weight of impurity in sample $(\mu \mathrm{g})$ : & 6.93 & 0.00 & 6.93 \\
\hline & Maximum corrected weight of impurity in sample $(\mu \mathrm{g})$ : & 7.34 & 0.42 & 7.76 \\
\hline \multirow{5}{*}{ v } & Measured concentration of impurity in sample $(\mu \mathrm{g} / \mathrm{ml})$ : & $1.06 \mathrm{E}+00$ & $6.43 \mathrm{E}-03$ & $\mathrm{v}$ \\
\hline & Uncorrected welght of impurity in sample $(\mu \mathrm{g})$ : & 54.06 & 0.33 & 54.39 \\
\hline & Weight of impurity in blank $(\mathrm{\mu g})$ : & $<0.10$ & $<0.10$ & \\
\hline & Minimum corrected weight of impurity in sample $(\mu \mathrm{g})$ : & 53.96 & 0.23 & 54.19 \\
\hline & Maximum corrected weight of impurity in sample $(\mu \mathrm{g})$ : & 54.06 & 0.33 & 54.39 \\
\hline \multicolumn{5}{|c|}{ Comments } \\
\hline & ed against RMAL 2157 official results of analyses by FCM & $\overline{114 / 2009}$ & & \\
\hline
\end{tabular}

\section{Fued c. Mortgomely}

$1-27-2010$

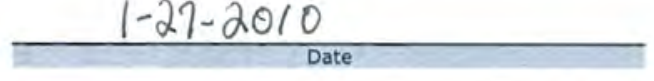


Data Report Form DRF-26B: Measurement of SIC Burn-Leach Defects or Impurities by Burn-Leach

\begin{tabular}{|c|c|}
\hline Procedure: & AGR-CHAR-DAM-26 Rev. 1 \\
\hline Operator: & Fred Montgomery \\
\hline Compact lot ID: & LEU09-0P2-Z \\
\hline Compact lot description: & AGR-2 UCO Variant, from G73J-14-93073A \\
\hline Compact ID numbers: & $067,081,019,008,032$ \\
\hline DRF filename: & IImc-agr|AGRILeachBurnLeach|LEU09-OP2-Z-DRF26R1_01.xIs \\
\hline
\end{tabular}

\begin{tabular}{|c|c|c|c|c|}
\hline \multirow{2}{*}{\multicolumn{2}{|c|}{$\begin{array}{r}\text { Mean average weight uranium per particle }(\mathrm{g}): \\
\text { Uncertainty in mean averaqe weight uranium per particle }(\mathrm{q}): \\
\end{array}$}} & \multicolumn{3}{|c|}{$3.96 \mathrm{E}-04$} \\
\hline & & \multicolumn{3}{|c|}{$8.10 \mathrm{E}-07$} \\
\hline & & First Leach & Second Leach & Total \\
\hline & Burn-leach solution ID: & $B 09081403$ & B09081703 & \\
\hline & Number of compacts: & & & \\
\hline & Total volume of leach solution $(\mathrm{ml})$ : & 50.5 & 52.8 & \\
\hline & Radiochemical laboratory analysis number: & $2157-018$ & $2157-023$ & \\
\hline & Measured uranium concentration $(\mu \mathrm{g} / \mathrm{ml})$ : & $3.09 \mathrm{E}-01$ & $3.43 \mathrm{E}-03$ & \\
\hline & Uncertainty in uranium concentration $(\mu \mathrm{g} / \mathrm{ml})$ : & $3.09 E-02$ & $3.43 \mathrm{E}-04$ & \\
\hline & Weight uranium leached $(g):$ & $1.56 \mathrm{E}-05$ & $1.81 E-07$ & $1.58 \mathrm{E}-05$ \\
\hline & Uncertainty in weight uranium leached $(\mathrm{g})$ : & $1.57 \mathrm{E}-06$ & $1.82 \mathrm{E}-08$ & $1.57 \mathrm{E}-06$ \\
\hline & Number of leached kernels: & 0.0 & 0.0 & 0.0 \\
\hline & Uncertainty in number of leached kernels: & 0.0 & 0.0 & 0.0 \\
\hline & 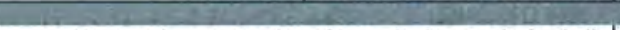 & & & \\
\hline \multirow{5}{*}{$\mathrm{Fe}$} & Measured concentration of impurity in sample $(\mu \mathrm{g} / \mathrm{ml})$ : & $4.42 E-01$ & $<4.12 \mathrm{E}-02$ & $\mathrm{Fe}$ \\
\hline & Uncorrected weight of impurity in sample $(\mu \mathrm{g})$ : & 22.32 & $<2.18$ & $<24.50$ \\
\hline & Weight of Impurity in blank $(\mu \mathrm{g})$ : & $<2.15$ & $<2.10$ & \\
\hline & Minimum corrected weight of impurity in sample $(\mu \mathrm{g})$ : & 20.17 & 0.00 & 20.17 \\
\hline & Maximum corrected weight of impurity in sample $(\mu \mathrm{g})$ : & 22.32 & 2.18 & 24.50 \\
\hline \multirow{5}{*}{$\mathrm{Cr}$} & Measured concentration of impurity in sample $(\mu \mathrm{g} / \mathrm{ml})$ : & $2.24 \mathrm{E}-02$ & $<2.00 \mathrm{E}-03$ & $\mathrm{Cr}$ \\
\hline & Uncorrected weight of impurity in sample $(\mu \mathrm{g})$ : & 1.13 & $<0.11$ & $<1.24$ \\
\hline & Weight of impurity in blank $(\mu \mathrm{g})$ : & $<0.10$ & $<0.10$ & \\
\hline & Minimum corrected weight of impurity in sample $(\mu \mathrm{g})$ : & 1.03 & 0.00 & 1.03 \\
\hline & Maximum corrected weight of impurity in sample $(\mu \mathrm{g})$ : & 1.13 & 0.11 & 1.24 \\
\hline \multirow{5}{*}{ Mn } & Measured concentration of impurity in sample $(\mu \mathrm{g} / \mathrm{ml})$ : & $<1.91 \mathrm{E}-03$ & $<1.91 \mathrm{E}-03$ & Mn \\
\hline & Uncorrected weight of impurity in sample $(\mu \mathrm{g})$ : & $<0.10$ & $<0.10$ & $<0.20$ \\
\hline & Weight of impurity in blank $(\mu \mathrm{g})$ : & $<0.10$ & $<0.10$ & \\
\hline & Minimum corrected weight of impurity in sample $(\mu \mathrm{g})$ : & 0.00 & 0.00 & 0.00 \\
\hline & Maximum corrected weight of impurity in sample $(\mu \mathrm{g})$ : & 0.10 & 0.10 & 0.20 \\
\hline \multirow{5}{*}{ Co } & Measured concentration of impurity in sample $(\mu \mathrm{g} / \mathrm{ml})$ : & $<1.62 \mathrm{E}-03$ & $<1.62 \mathrm{E}-03$ & Co \\
\hline & Uncorrected weight of impurity in sample $(\mu \mathrm{g})$ : & $<0.08$ & $<0.09$ & $<0.17$ \\
\hline & Weight of impurity in blank $(\mu \mathrm{g})$ : & $<0.08$ & $<0.08$ & \\
\hline & Minimum corrected weight of impurity in sample $(\mu \mathrm{g})$ : & 0.00 & 0.00 & 0.00 \\
\hline & Maximum corrected weight of impurity in sample $(\mu \mathrm{g})$ : & 0.08 & 0.09 & 0.17 \\
\hline \multirow{5}{*}{$\mathbf{N i}$} & Measured concentration of impurity in sample $(\mu \mathrm{g} / \mathrm{ml})$ : & $7.44 \mathrm{E}-02$ & $<8.00 \mathrm{E}-03$ & $\mathrm{Ni}$ \\
\hline & Uncorrected weight of impurity in sample $(\mu \mathrm{g})$ : & 3.76 & $<0.42$ & $<4.18$ \\
\hline & Weight of impurity in blank $(\mu \mathrm{g})$ : & $<0.42$ & $<0.41$ & \\
\hline & Minimum corrected weight of impurity in sample $(\mu \mathrm{g})$ : & 3.34 & 0.00 & 3.34 \\
\hline & Maximum corrected weight of impurity in sample $(\mu \mathrm{g})$ : & 3.76 & 0.42 & 4.18 \\
\hline \multirow{5}{*}{$\mathbf{C a}$} & Measured concentration of impurity in sample $(\mu \mathrm{g} / \mathrm{ml})$ : & $3.96 \mathrm{E}+00$ & $5.26 \mathrm{E}-01$ & $\mathrm{Ca}$ \\
\hline & Uncorrected weight of impurity in sample $(\mu \mathrm{g})$ : & 199.98 & 27.77 & 227.75 \\
\hline & Weight of impurity in blank $(\mu \mathrm{g})$ : & 9.92 & 97.41 & 5 \\
\hline & Minimum corrected weight of impurity in sample $(\mu \mathrm{g})$ : & 190.06 & 0.00 & 190.06 \\
\hline & Maximum corrected weight of impurity in sample $(\mu \mathrm{g})$ : & 190.06 & 0.00 & 190.06 \\
\hline \multirow{5}{*}{ Al } & Measured concentration of impurity in sample $(\mu \mathrm{g} / \mathrm{ml})$ : & $1.61 E+00$ & $3.57 \mathrm{E}-02$ & Al \\
\hline & Uncorrected weight of impurity in sample $(\mu \mathrm{g})$ : & 81.31 & 1.88 & 83.19 \\
\hline & Weight of impurity in blank $(\mu \mathrm{g})$ : & 3.05 & 1.28 & \\
\hline & Minimum corrected weight of impurity in sample $(\mu \mathrm{g})$ : & 78.25 & 0.61 & 78.86 \\
\hline & Maximum corrected weight of impurity in sample $(\mu \mathrm{g})$ : & 78.25 & 0.61 & 78.86 \\
\hline \multirow{5}{*}{$\mathbf{T i}$} & Measured concentration of impurity in sample $(\mu \mathrm{g} / \mathrm{ml})$ : & $1.61 \mathrm{E}-01$ & $<8.00 E-03$ & $\mathrm{Ti}$ \\
\hline & Uncorrected weight of impurity in sample $(\mu \mathrm{g})$ : & 8.13 & $<0.42$ & $<8.55$ \\
\hline & Weight of impurity in blank $(\mu \mathrm{g})$ : & $<0.42$ & $<0.41$ & \\
\hline & Minimum corrected weight of impurity in sample $(\mu \mathrm{g})$ : & 7.71 & 0.00 & 7.71 \\
\hline & Maximum corrected weight of impurity in sample $(\mu \mathrm{g})$ : & 8.13 & 0.42 & 8.55 \\
\hline \multirow{5}{*}{$\mathbf{v}$} & Measured concentration of impurity in sample $(\mu \mathrm{g} / \mathrm{ml})$ : & $1.20 E+00$ & $7.76 \mathrm{E}-03$ & v \\
\hline & Uncorrected weight of impurity in sample $(\mu \mathrm{g})$ : & 60.60 & 0.41 & 61.01 \\
\hline & Weight of impurity in blank $(\mu \mathrm{g})$ : & $<0.10$ & $<0.10$ & \\
\hline & Minimum corrected weight of impurity in sample $(\mu \mathrm{g})$ : & 60.50 & 0.31 & 60.80 \\
\hline & Maximum corrected weight of impurity in sample $(\mu \mathrm{g})$ : & 60.60 & 0.41 & 61.01 \\
\hline
\end{tabular}

Comments

Data checked against RMAL 2157 official results of analyses by FCM on 10/14/2009

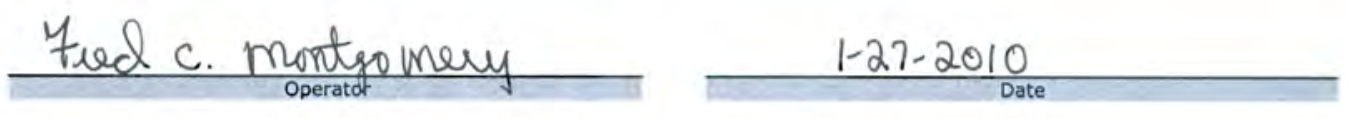


Data Report Form DRF-26B: Measurement of SiC Burn-Leach Defects or Impurities by Burn-Leach

\begin{tabular}{|c|c|}
\hline $\begin{array}{c}\text { Procedure: } \\
\text { Operator: } \\
\text { F }\end{array}$ & $\begin{array}{l}\text { AGR-CHAR-DAM-26 Rev. } 1 \\
\text { Fred Montgomery }\end{array}$ \\
\hline Compact lot ID: L & LEU09-OP2-Z \\
\hline Compact lot description: & $A G R-2$ UCO Variant, from $G$ \\
\hline Compact ID numbers: & 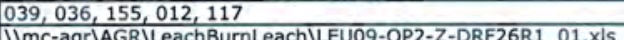 \\
\hline
\end{tabular}

\begin{tabular}{|c|c|c|c|c|}
\hline \multirow{2}{*}{\multicolumn{2}{|c|}{$\begin{array}{l}\text { Mean average weight uranium per particle }(\mathrm{g}) \text { : } \\
\text { Uncertainty in mean average weight uranium per particle }(\mathrm{q}): \\
\end{array}$}} & \multicolumn{3}{|c|}{$\begin{array}{l}3.96 \mathrm{E}-04 \\
8.10 \mathrm{E}-07\end{array}$} \\
\hline & & First Leach & Second Leach & Total \\
\hline & Burn-leach solution ID: & B09081404 & B09081704 & \\
\hline & Number of compacts: & & & \\
\hline & Total volume of leach solution $(\mathrm{ml}):$ & 50.0 & 52.5 & \\
\hline & Radiochemical laboratory analysis number: & $2157-019$ & $2157-024$ & \\
\hline & Measured uranium concentration $(\mu \mathrm{g} / \mathrm{ml})$ : & $2.86 \mathrm{E}-01$ & $3.98 \mathrm{E}-03$ & \\
\hline & Uncertainty in uranium concentration $(\mu \mathrm{g} / \mathrm{ml})$ : & $2.86 E-02$ & $3.98 \mathrm{E}-04$ & \\
\hline & $\begin{array}{c}\text { Weight uranium leached }(\mathrm{g}): \\
\end{array}$ & $1.43 \mathrm{E}-05$ & $2.09 E-07$ & $1.45 \mathrm{E}-05$ \\
\hline & Uncertainty in weight uranium leached $(\mathrm{g})$ : & $1.44 \mathrm{E}-06$ & $2.11 \mathrm{E}-08$ & $1.44 \mathrm{E}-06$ \\
\hline & Number of leached kernels: & 0.0 & 0.0 & 0.0 \\
\hline & Uncertainty in number of leached kernels: & 0.0 & 0.0 & 0.0 \\
\hline \multirow{5}{*}{$\mathrm{Fe}$} & Measured concentration of impurity in sample $(\mu \mathrm{g} / \mathrm{ml})$ : & $5.90 \mathrm{E}-02$ & $<4.12 \mathrm{E}-02$ & $\mathbf{F e}$ \\
\hline & Uncorrected weight of impurity in sample $(\mu \mathrm{g})$ : & 2.95 & $<2.16$ & $<5.11$ \\
\hline & Weight of impurity in blank $(\mu \mathrm{g})$ : & $<2.15$ & $<2.10$ & \\
\hline & Minimum corrected weight of impurity in sample $(\mu \mathrm{g})$ : & 0.80 & 0.00 & 0.80 \\
\hline & Maximum corrected weight of impurity in sample $(\mu g)$ : & 2.95 & 2.16 & 5.11 \\
\hline \multirow{5}{*}{ Cr } & Measured concentration of impurity in sample $(\mathrm{\mu g} / \mathrm{ml})$ : & $2.11 \mathrm{E}-02$ & $<2.00 \mathrm{E}-03$ & $\mathrm{Cr}$ \\
\hline & Uncorrected weight of impurity in sample $(\mu \mathrm{g})$ : & 1.06 & $<0.11$ & $<1,16$ \\
\hline & Weight of impurity in blank $(\mu g)$ : & $<0.10$ & $<0.10$ & \\
\hline & Minimum corrected weight of impurity in sample $(\mu \mathrm{g})$ : & 0.95 & 0.00 & 0.95 \\
\hline & Maximum corrected weight of impurity in sample $(\mu g)$ : & 1.06 & 0.11 & 1.16 \\
\hline \multirow{5}{*}{ Mn } & Measured concentration of impurity in sample $(\mathrm{\mu g} / \mathrm{ml})$ : & $<1.91 \mathrm{E}-03$ & $<1.91 \mathrm{E}-03$ & $\mathrm{Mn}$ \\
\hline & Uncorrected weight of impurity in sample $(\mu g)$ : & $<0.10$ & $<0.10$ & $<0.20$ \\
\hline & Weight of impurity in blank $(\mu g)$ : & $<0.10$ & $<0.10$ & \\
\hline & Minimum corrected weight of impurity in sample $(\mu g)$ : & 0.00 & 0.00 & 0.00 \\
\hline & Maximum corrected weight of impurity in sample $(\mu q)$ : & 0.10 & 0.10 & 0.20 \\
\hline \multirow{5}{*}{ Co } & Measured concentration of impurity in sample $(\mu \mathrm{g} / \mathrm{ml})$ : & $<1.62 \mathrm{E}-03$ & $<1.62 \mathrm{E}-03$ & Co \\
\hline & Uncorrected weight of impurity in sample $(\mu \mathrm{g})$ : & $<0.08$ & $<0.09$ & $<0.17$ \\
\hline & Weight of impurity in blank $(\mu \mathrm{g})$ : & $<0.08$ & $<0.08$ & \\
\hline & Minimum corrected weight of impurity in sample $(\mu g)$ : & 0.00 & 0.00 & 0.00 \\
\hline & Maximum corrected weight of impurity in sample $(\mu g)$ : & 0.08 & 0.09 & 0.17 \\
\hline \multirow{5}{*}{$\mathrm{Ni}$} & Measured concentration of impurity in sample $(\mathrm{\mu g} / \mathrm{ml})$ : & $1.51 \mathrm{E}-02$ & $<8.00 \mathrm{E}-03$ & $\mathrm{Ni}$ \\
\hline & Uncorrected weight of impurity in sample $(\mu \mathrm{g})$ : & 0.76 & $<0.42$ & $<1.18$ \\
\hline & Weight of impurity in blank $(\mu \mathrm{g})$ : & $<0.42$ & $<0.41$ & \\
\hline & Minimum corrected weight of impurity in sample $(\mu g)$ : & 0.34 & 0.00 & 0.34 \\
\hline & Maximum corrected weight of impurity in sample $(\mu g)$ : & 0.76 & 0.42 & 1.18 \\
\hline \multirow{5}{*}{ Ca } & Measured concentration of impurity in sample $(\mu \mathrm{g} / \mathrm{ml})$ : & $2.36 \mathrm{E}+00$ & $4.92 \mathrm{E}-01$ & $\mathrm{Ca}$ \\
\hline & Uncorrected weight of Impurity in sample $(\mu g)$ : & 118.00 & 25.83 & 143.83 \\
\hline & Weight of impurity in blank $(\mu g)$ : & 9.92 & 97.41 & \\
\hline & Minimum corrected weight of impurity in sample $(\mu g)$ : & 108.08 & 0.00 & 108.08 \\
\hline & Maximum corrected weight of impurity in sample $(\mu g)$ : & 108.08 & 0.00 & 108.08 \\
\hline \multirow{5}{*}{ Al } & Measured concentration of impurity in sample $(\mu \mathrm{g} / \mathrm{ml})$ : & $1.60 \mathrm{E}+00$ & $5.00 \mathrm{E}-02$ & Al \\
\hline & Uncorrected weight of impurity in sample $(\mu g)$ : & 80.00 & 2.63 & 82.63 \\
\hline & Weight of impurity in blank $(\mu g)$ : & 3.05 & 1.28 & \\
\hline & Minimum corrected weight of impurity in sample $(\mu g)$ : & 76.95 & 1.35 & 78.30 \\
\hline & Maximum corrected weight of impurity in sample ( $\mu \mathrm{g})$ : & 76.95 & 1.35 & 78.30 \\
\hline \multirow{5}{*}{$\mathbf{T i}$} & Measured concentration of impurity in sample $(\mu \mathrm{g} / \mathrm{ml})$ : & $9.27 \mathrm{E}-02$ & $<8.00 \mathrm{E}-03$ & $\mathrm{Ti}$ \\
\hline & Uncorrected weight of impurity in sample $(\mu \mathrm{g})$ : & 4.64 & $<0.42$ & $<5.06$ \\
\hline & Weight of impurity in blank $(\mu \mathrm{g})$ : & $<0.42$ & $<0.41$ & \\
\hline & Minimum corrected weight of impurity in sample $(\mu g)$ : & 4.22 & 0.00 & 4.22 \\
\hline & Maximum corrected weight of impurity in sample $(\mu g)$ : & 4.64 & 0.42 & 5.06 \\
\hline \multirow{5}{*}{ v } & Measured concentration of Impurity in sample $(\mu \mathrm{g} / \mathrm{ml})$ : & $1.02 E+00$ & $7.92 \mathrm{E}-03$ & $\mathrm{v}$ \\
\hline & Uncorrected weight of impurity in sample $(\mu \mathrm{g})$ : & 51.00 & 0.42 & 51.42 \\
\hline & Weight of impurity in blank $(\mu \mathrm{g})$ : & $<0.10$ & $<0.10$ & \\
\hline & Minimum corrected weight of impurity in sample $(\mu g)$ : & 50.90 & 0.31 & 51.21 \\
\hline & Maximum corrected weight of impurity in sample $(\mu g)$ : & 51.00 & 0.42 & 51.42 \\
\hline \multicolumn{5}{|c|}{ Comments } \\
\hline \multicolumn{5}{|c|}{ Data checked against RMAL 2157 official results of analyses by FCM on 10/14/2009 } \\
\hline
\end{tabular}


Data Report Form DRF-26B: Measurement of SIC Burn-Leach Defects or Impurities by Burn-Leach

\begin{tabular}{|c|c|}
\hline Procedure: & AGR-CHAR-DAM-26 Rev. 1 \\
\hline Operator: & Fred Montgomery \\
\hline Compact lot ID: & LEU09-OP2-Z \\
\hline Compact lot description: & AGR-2 UCO Variant, from G73J-14-93073A \\
\hline Compact ID numbers: & $031,149,093,147,072$ \\
\hline DRF filename: & Ml|mc-aqr|AGR|LeachBurnLeach|LEU09-OP2-Z-DRF26R1_01.xis \\
\hline
\end{tabular}

\begin{tabular}{|c|c|c|c|c|}
\hline \multirow{2}{*}{\multicolumn{2}{|c|}{$\begin{array}{r}\text { Mean average weight uranium per particle }(\mathrm{q}) \text { : } \\
\text { Uncertainty in mean averaqe weight uranium per particle }(\mathrm{q}) \text { : }\end{array}$}} & \multicolumn{3}{|c|}{$\frac{3.96 E-04}{8.10 E-07}$} \\
\hline & & \multicolumn{3}{|c|}{$8.10 \mathrm{E}-07$} \\
\hline & & First Leach & Second Leach & Total \\
\hline & Burn-leach solution ID: & B09081405 & B09081705 & \\
\hline & Number of compacts: & & & \\
\hline & Total volume of leach solution $(\mathrm{ml})$ : & 52.0 & 52.5 & \\
\hline & Radiochemical laboratory analysis number: & $2157-020$ & $2157-025$ & \\
\hline & Measured uranium concentration $(\mu \mathrm{g} / \mathrm{ml})$ : & $2.70 \mathrm{E}-01$ & $2.86 \mathrm{E}-03$ & \\
\hline & Uncertainty in uranium concentration $(\mu \mathrm{g} / \mathrm{ml})$ : & $2.70 \mathrm{E}-02$ & $2.86 \mathrm{E}-04$ & \\
\hline & Weight uranium leached $(g):$ & $1.40 \mathrm{E}-05$ & $1.50 \mathrm{E}-07$ & $1.42 \mathrm{E}-05$ \\
\hline & Uncertainty in weight uranium leached $(g)$ : & $1.41 \mathrm{E}-06$ & $1.51 \mathrm{E}-08$ & $1.42 \mathrm{E}-06$ \\
\hline & Number of leached kernels: & 0.0 & 0.0 & 0.0 \\
\hline & Uncertainty in number of leached kernels: & 0.0 & 0.0 & 0.0 \\
\hline \multirow{5}{*}{$\mathrm{Fe}$} & Measured concentration of impurity in sample $(\mu \mathrm{g} / \mathrm{ml})$ : & $5.92 E-02$ & $<4.12 \mathrm{E}-02$ & $\mathbf{F e}$ \\
\hline & Uncorrected weight of impurity in sample $(\mu \mathrm{g})$ : & 3.08 & $<2.16$ & $<5.24$ \\
\hline & Weight of impurity in blank $(\mu \mathrm{g})$ : & $<2.15$ & $<2.10$ & sine \\
\hline & Minimum corrected weight of impurity in sample $(\mu \mathrm{g})$ : & 0.93 & 0.00 & 0.93 \\
\hline & Maximum corrected weight of impurity in sample $(\mu \mathrm{g})$ : & 3.08 & 2.16 & 5.24 \\
\hline \multirow{5}{*}{$\mathrm{Cr}$} & Measured concentration of impurity in sample $(\mu \mathrm{g} / \mathrm{ml})$ : & 2.01E-02 & $<2.00 \mathrm{E}-03$ & $\mathrm{Cr}$ \\
\hline & Uncorrected weight of impurity in sample $(\mu \mathrm{g})$ : & 1.05 & $<0.11$ & $<1.15$ \\
\hline & Weight of impurity in blank $(\mu \mathrm{g})$ : & $<0.10$ & $<0.10$ & \\
\hline & Minimum corrected weight of impurity in sample $(\mu \mathrm{g})$ : & 0.94 & 0.00 & 0.94 \\
\hline & Maximum corrected weight of impurity in sample $(\mu \mathrm{g})$ : & 1.05 & 0.11 & 1.15 \\
\hline \multirow{5}{*}{ Mn } & Measured concentration of impurity in sample $(\mu \mathrm{g} / \mathrm{ml})$ : & $<1.91 \mathrm{E}-03$ & $<1.91 \mathrm{E}-03$ & Mn \\
\hline & Uncorrected weight of impurity in sample $(\mu \mathrm{g})$ : & $<0.10$ & $<0.10$ & $<0.20$ \\
\hline & Weight of impurity in blank $(\mu \mathrm{g})$ : & $<0.10$ & $<0.10$ & = \\
\hline & Minimum corrected weight of impurity in sample $(\mu \mathrm{g})$ : & 0.00 & 0.00 & 0.00 \\
\hline & Maximum corrected weight of impurity in sample $(\mu \mathrm{g})$ : & 0.10 & 0.10 & 0.20 \\
\hline \multirow{5}{*}{ Co } & Measured concentration of impurity in sample $(\mu \mathrm{g} / \mathrm{ml})$ : & $<1.62 \mathrm{E}-03$ & $<1.62 \mathrm{E}-03$ & Co \\
\hline & Uncorrected weight of impurity in sample $(\mu \mathrm{g})$ : & $<0.08$ & $<0.09$ & $<0.17$ \\
\hline & Weight of impurity in blank $(\mu \mathrm{g})$ : & $<0.08$ & $<0.08$ & \\
\hline & Minimum corrected welght of impurity in sample $(\mu \mathrm{g})$ : & 0.00 & 0.00 & 0.00 \\
\hline & Maximum corrected weight of impurity in sample $(\mu \mathrm{g})$ : & 0.08 & 0.09 & 0.17 \\
\hline \multirow{5}{*}{$\mathrm{Ni}$} & Measured concentration of impurity in sample $(\mu \mathrm{g} / \mathrm{ml})$ : & $3.85 E-02$ & $<8.00 \mathrm{E}-03$ & $\mathrm{Ni}$ \\
\hline & Uncorrected weight of impurity in sample $(\mu \mathrm{g})$ : & 2.00 & $<0.42$ & $<2.42$ \\
\hline & Weight of impurity in blank $(\mu \mathrm{g})$ : & $<0.42$ & $<0.41$ & \\
\hline & Minimum corrected weight of impurity in sample $(\mu \mathrm{g})$ : & 1.58 & 0.00 & 1.58 \\
\hline & Maximum corrected weight of impurity in sample $(\mu \mathrm{g})$ : & 2.00 & 0.42 & 2.42 \\
\hline \multirow{5}{*}{$\mathbf{C a}$} & Measured concentration of impurity in sample $(\mu \mathrm{g} / \mathrm{ml})$ : & $3.19 \mathrm{E}+00$ & $1.49 \mathrm{E}+00$ & $\mathrm{Ca}$ \\
\hline & Uncorrected weight of impurity in sample $(\mu \mathrm{g})$ : & 165.88 & 78.23 & 244.11 \\
\hline & Weight of impurity in blank $(\mu \mathrm{g})$ : & 9.92 & 97.41 & \\
\hline & Minimum corrected weight of impurity in sample $(\mu \mathrm{g})$ : & 155.96 & 0.00 & 155.96 \\
\hline & Maximum corrected weight of impurity in sample $(\mu \mathrm{g})$ : & 155.96 & 0.00 & 155.96 \\
\hline \multirow{5}{*}{ Al } & Measured concentration of impurity in sample $(\mu \mathrm{g} / \mathrm{ml})$ : & $1.52 E+00$ & $3.25 E-02$ & Al \\
\hline & Uncorrected weight of impurity in sample $(\mu \mathrm{g})$ : & 79.04 & 1.71 & 80.75 \\
\hline & Weight of impurity in blank $(\mu \mathrm{g})$ : & 3.05 & 1.28 & \\
\hline & Minimum corrected weight of impurity in sample $(\mu \mathrm{g})$ : & 75.99 & 0.43 & 76.42 \\
\hline & Maximum corrected weight of impurity in sample $(\mu \mathrm{g})$ : & 75.99 & 0.43 & 76.42 \\
\hline \multirow{5}{*}{$\mathbf{T i}$} & Measured concentration of impurity in sample $(\mu \mathrm{g} / \mathrm{ml})$ : & $1.34 \mathrm{E}-01$ & $<8.00 \mathrm{E}-03$ & $\mathrm{Ti}$ \\
\hline & Uncorrected weight of impurity in sample $(\mu \mathrm{g})$ : & 6.97 & $<0.42$ & $<7.39$ \\
\hline & Weight of impurity in blank $(\mu \mathrm{g})$ : & $<0.42$ & $<0.41$ & \\
\hline & Minimum corrected weight of impurity in sample $(\mu \mathrm{g})$ : & 6.55 & 0.00 & 6.55 \\
\hline & Maximum corrected weight of impurity in sample $(\mu \mathrm{g})$ : & 6.97 & 0.42 & 7.39 \\
\hline \multirow{5}{*}{$\mathbf{v}$} & Measured concentration of impurity in sample $(\mu \mathrm{g} / \mathrm{ml})$ : & $1.03 E+00$ & $5.41 \mathrm{E}-03$ & $\mathbf{v}$ \\
\hline & Uncorrected weight of impurity in sample $(\mu \mathrm{g})$ : & 53.56 & 0.28 & 53.84 \\
\hline & Weight of impurity in blank $(\mu \mathrm{g})$ : & $<0.10$ & $<0.10$ & \\
\hline & Minimum corrected weight of impurity in sample $(\mu \mathrm{g})$ : & 53.46 & 0.18 & 53.64 \\
\hline & Maximum corrected weight of impurity in sample $(\mu \mathrm{g})$ : & 53.56 & 0.28 & 53.84 \\
\hline
\end{tabular}

Data checked against RMAL 2157 official results of analyses by FCM on 10/14/2009

Data checked against RMAL 2157 official results of analyses by FCM on 10/14/2009 
Data Report Form DRF-26B: Measurement of SiC Burn-Leach Defects or Impurities by Burn-Leach

\begin{tabular}{|r|l|}
\hline Procedure: & AGR-CHAR-DAM-26 Rev. 1 \\
\hline Operator: & Fred Montgomery \\
\hline Compact lot ID: & LEU09-OP2-Z \\
\hline Compact lot description: & AGR-2 UCO Variant, from G73J-14-93073A \\
\hline Compact ID numbers: & Burn-Leach Blank \\
\hline DRF filename: & IImC-agr\AGR\LeachBurnLeach \LEU09-OP2-Z-DRF26R1_01.xIs \\
\hline
\end{tabular}

\begin{tabular}{|c|c|c|c|c|}
\hline \multicolumn{2}{|r|}{ Mean average weight uranium per particle $(g):$} & \multicolumn{3}{|c|}{$3.96 \mathrm{E}-04$} \\
\hline & Uncertainty in mean average weight uranium per particle $(\mathrm{g})$ : & \multicolumn{3}{|c|}{$8.10 \mathrm{E}-07$} \\
\hline & & First Leach & Second Leach & Total \\
\hline & Burn-leach solution ID: & B09081402 & B09081702 & \\
\hline & Number of compacts: & \multicolumn{2}{|c|}{ None } & \\
\hline & Total volume of leach solution $(\mathrm{ml})$ : & 52.2 & 51.0 & \\
\hline & Radiochemical laboratory analysis number: & $2157-017$ & $2157-022$ & \\
\hline & Measured uranium concentration $(\mu \mathrm{g} / \mathrm{ml})$ : & $<2.00 \mathrm{E}-04$ & $<2.00 \mathrm{E}-04$ & \\
\hline \multicolumn{5}{|c|}{ Uncertainty in uranium concentration $(\mu \mathrm{g} / \mathrm{ml})$ : } \\
\hline & Weight uranium leached $(\mathrm{g})$ : & $<1.04 \mathrm{E}-08$ & $<1.02 \mathrm{E}-08$ & $<2.06 \mathrm{E}-08$ \\
\hline \multicolumn{5}{|c|}{ Uncertainty in weight uranium leached $(\mathrm{g})$ : } \\
\hline & Number of leached kernels: & 0.0 & 0.0 & 0.0 \\
\hline \multicolumn{5}{|c|}{ Uncertainty in number of leached kernels: } \\
\hline \multirow{2}{*}{$\mathbf{F e}$} & Measured concentration $(\mu \mathrm{g} / \mathrm{ml})$ : & $<4.12 \mathrm{E}-02$ & $<4.12 \mathrm{E}-02$ & $\mathrm{Fe}$ \\
\hline & Total weight of leached impurity $(\mu \mathrm{g})$ : & $<2.15$ & $<2.10$ & $<4.25$ \\
\hline \multirow{2}{*}{$\mathbf{C r}$} & Measured concentration $(\mu \mathrm{g} / \mathrm{ml})$ : & $<2.00 \mathrm{E}-03$ & $<2.00 \mathrm{E}-03$ & $\mathrm{Cr}$ \\
\hline & Total weight of leached impurity $(\mu \mathrm{g})$ : & $<0.10$ & $<0.10$ & $<0.21$ \\
\hline \multirow{2}{*}{ Mn } & Measured concentration $(\mu \mathrm{g} / \mathrm{ml})$ : & $<1.91 \mathrm{E}-03$ & $<1.91 \mathrm{E}-03$ & Mn \\
\hline & Total weight of leached impurity $(\mu g)$ : & $<0.10$ & $<0.10$ & $<0.20$ \\
\hline \multirow{2}{*}{ Co } & Measured concentration $(\mu \mathrm{g} / \mathrm{ml})$ : & $<1.62 \mathrm{E}-03$ & $<1.62 \mathrm{E}-03$ & Co \\
\hline & Total weight of leached impurity $(\mu \mathrm{g})$ : & $<0.08$ & $<0.08$ & $<0.17$ \\
\hline \multirow{2}{*}{ Ni } & Measured concentration $(\mu \mathrm{g} / \mathrm{ml})$ : & $<8.00 \mathrm{E}-03$ & $<8.00 \mathrm{E}-03$ & $\mathrm{Ni}$ \\
\hline & Total weight of leached impurity $(\mu \mathrm{g})$ : & $<0.42$ & $<0.41$ & $<0.83$ \\
\hline \multirow{2}{*}{$\mathbf{C a}$} & Measured concentration $(\mu \mathrm{g} / \mathrm{ml})$ : & $1.90 \mathrm{E}-01$ & $1.91 \mathrm{E}+00$ & $\mathrm{Ca}$ \\
\hline & Total weight of leached impurity $(\mu \mathrm{g})$ : & 9.92 & 97.41 & 107.33 \\
\hline \multirow{2}{*}{ Al } & Measured concentration $(\mu \mathrm{g} / \mathrm{ml})$ : & 5.85E-02 & $2.50 E-02$ & Al \\
\hline & Total weight of leached impurity $(\mu \mathrm{g})$ : & 3.05 & 1.28 & 4.33 \\
\hline \multirow{2}{*}{$\mathbf{T i}$} & Measured concentration $(\mu \mathrm{g} / \mathrm{ml})$ : & $<8.00 \mathrm{E}-03$ & $<8.00 \mathrm{E}-03$ & $\mathrm{Ti}$ \\
\hline & Total weight of leached impurity $(\mu \mathrm{g})$ : & $<0.42$ & $<0.41$ & $<0.83$ \\
\hline \multirow{2}{*}{$\mathbf{v}$} & Measured concentration $(\mu \mathrm{g} / \mathrm{ml})$ : & $<2.00 \mathrm{E}-03$ & $\leq 2.00 E-03$ & $\mathbf{v}$ \\
\hline & Total weight of leached impurity $(\mu \mathrm{g})$ : & $<0.10$ & $<0.10$ & $<0.21$ \\
\hline
\end{tabular}

\section{Comments}

Data checked against RMAL 2157 official results of analyses by FCM on 10/14/2009

Feed c. nontroneney
$1-27.2010$

Date 
Data Report Form DRF-26A: Measurement of U Contamination or Impuritles by Deconsollidation Leach

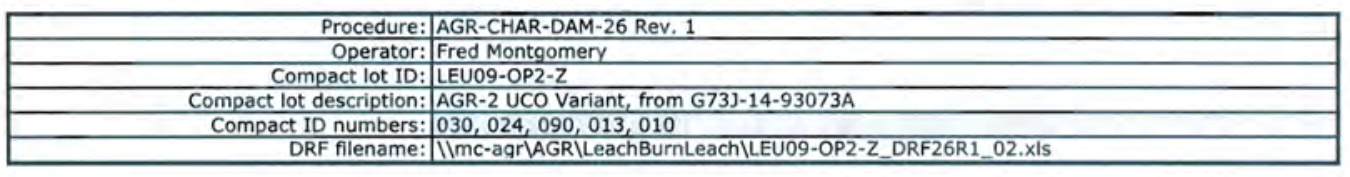

\begin{tabular}{|c|c|c|c|c|}
\hline \multicolumn{2}{|r|}{$\begin{array}{r}\text { Mean average weight uranium per particle }(g) \text { : } \\
\text { Uncertainty in mean averaqe weight uranium per particle }(q) \text { : }\end{array}$} & \multicolumn{3}{|c|}{$\begin{array}{l}3.96 E-04 \\
8.10 E-07\end{array}$} \\
\hline & & First Leach & Second Leach & Total \\
\hline & Deconsolidation-leach solution ID: & L09081002 & L09081202 & \\
\hline & Number of compacts: & & & \\
\hline & Total volume of leach solution (ml): & 138.0 & 128.0 & \\
\hline & Radiochemical laboratory analysis number: & $2157-002$ & $2157-007$ & \\
\hline & Measured uranium concentration $(\mathrm{\mu g} / \mathrm{ml})$ : & $6.16 \mathrm{E}-02$ & $6.89 \mathrm{E}-03$ & \\
\hline & Uncertainty in uranium concentration $(\mu \mathrm{g} / \mathrm{ml})$ : & $6.16 \mathrm{E}-03$ & $6.89 \mathrm{E}-04$ & \\
\hline & Weight uranium leached $(\mathrm{g})$ : & $8.50 \mathrm{E}-06$ & $8.82 \mathrm{E}-07$ & $9.38 \mathrm{E}-06$ \\
\hline & Uncertainty in weight uranium leached $(\mathrm{g})$ : & $8.51 \mathrm{E}-07$ & $8.83 E-08$ & $8.56 \mathrm{E}-07$ \\
\hline & $\begin{array}{r}\text { Effective number of exposed kernels: } \\
\end{array}$ & 0.0 & 0.0 & 0.0 \\
\hline & Uncertainty in effective number of exposed kernels: & 0.0 & 0.0 & 0.0 \\
\hline \multirow{5}{*}{$\mathbf{F e}$} & Measured concentration of impurity in sample $(\mu \mathrm{g} / \mathrm{ml})$ : & $<4.12 \mathrm{E}-02$ & $<4.12 \mathrm{E}-02$ & $\mathbf{F e}$ \\
\hline & Uncorrected weight of impurity in sample $(\mu \mathrm{g})$ : & $<5.69$ & $<5.27$ & $<10.96$ \\
\hline & Weight of impurity in blank $(\mu \mathrm{g})$ : & $<7.05$ & $<5.15$ & \\
\hline & Minimum corrected weight of impurity in sample $(\mu g)$ : & 0.00 & 0.00 & 0.00 \\
\hline & Maximum corrected weight of impurity in sample $(\mu g)$ : & 5.69 & 5.27 & 10.96 \\
\hline \multirow{4}{*}{$\mathbf{C r}$} & Measured concentration of impurity in sample $(\mathrm{\mu g} / \mathrm{ml})$ : & $8.42 E-03$ & $5.75 \mathrm{E}-03$ & $\mathrm{Cr}$ \\
\hline & Uncorrected weight of impurity in sample $(\mu \mathrm{g})$ : & 1.16 & 0.74 & 1.90 \\
\hline & Weight of impurity in blank $(\mu \mathrm{g})$ : & $<0.34$ & $<0.25$ & \\
\hline & $\begin{array}{l}\text { Minimum corrected weight of impurity in sample }(\mu g) \text { : } \\
\text { Maximum corrected weight of impurity in sample }(\mu g) \text { : }\end{array}$ & 0.82 & 0.49 & 1.31 \\
\hline \multirow{5}{*}{ Mn } & Maximum corrected weight of impurity in sample $(\mu \mathrm{g})$ : & 1.16 & 0.74 & 1.90 \\
\hline & $\begin{array}{c}\text { Measured concentration of impurity in sample }(\mu \mathrm{g} / \mathrm{ml}) \text { : } \\
\text { Uncorrected weight of impurity in sample }(\mu \mathrm{g}) \text { : }\end{array}$ & $\frac{<1.91 \mathrm{E}-03}{<0.26}$ & $\frac{<1.91 \mathrm{E}-03}{<0.24}$ & $\frac{\mathrm{Mn}}{<0.51}$ \\
\hline & Weight of impurity in blank $(\mu \mathrm{g})$ : & $<0.33$ & $<0.24$ & \\
\hline & Minimum corrected weight of impurity in sample $(\mu g)$ : & 0.00 & 0.00 & 0.00 \\
\hline & Maximum corrected weight of impurity in sample $(\mu g)$ : & 0.26 & 0.24 & 0.51 \\
\hline \multirow{5}{*}{ Co } & Measured concentration of impurity in sample $(\mathrm{\mu g} / \mathrm{ml})$ : & $<1.62 \mathrm{E}-03$ & $<1.62 \mathrm{E}-03$ & Co \\
\hline & Uncorrected weight of impurity in sample $(\mu \mathrm{g})$ : & $<0.22$ & $<0.21$ & $<0.43$ \\
\hline & Weight of impurity in blank $(\mu \mathrm{g})$ : & $<0.28$ & $<0.20$ & \\
\hline & Minimum corrected weight of Impurity in sample $(\mu g)$ : & 0.00 & 0.00 & 0.00 \\
\hline & Maximum corrected weight of Impurity in sample $(\mu \mathrm{g})$ : & 0.22 & 0.21 & 0.43 \\
\hline \multirow{5}{*}{ Ni } & Measured concentration of impurity in sample $(\mu \mathrm{g} / \mathrm{ml})$ : & $<8.00 \mathrm{E}-03$ & $<8.00 \mathrm{E}-03$ & $\mathrm{Ni}$ \\
\hline & Uncorrected weight of impurity in sample $(\mu \mathrm{g})$ : & $<1.10$ & $<1.02$ & $<2.13$ \\
\hline & Weight of impurity in blank $(\mu \mathrm{g})$ : & $<1.37$ & $<1.00$ & \\
\hline & Minimum corrected weight of impurity in sample $(\mu \mathrm{g})$ : & 0.00 & 0.00 & 0.00 \\
\hline & Maximum corrected weight of impurity in sample $(\mu \mathrm{g})$ : & 1.10 & 1.02 & 2.13 \\
\hline \multirow{5}{*}{$\mathrm{Ca}$} & Measured concentration of impurity in sample $(\mu \mathrm{g} / \mathrm{ml})$ : & $2.03 E+00$ & $8.89 \mathrm{E}-01$ & $\mathrm{Ca}$ \\
\hline & Uncorrected weight of impurity in sample $(\mu \mathrm{g})$ : & 280.14 & 113.79 & 393.93 \\
\hline & Weight of impurity in blank $(\mu \mathrm{g})$ : & 162.28 & 115.63 & \\
\hline & Minimum corrected weight of impurity in sample $(\mu \mathrm{g})$ : & 117.86 & 0.00 & 117.86 \\
\hline & Maximum corrected weight of impurity in sample $(\mu g)$ : & 117.86 & 0.00 & 117.86 \\
\hline \multirow{5}{*}{ Al } & Measured concentration of impurity in sample $(\mu \mathrm{g} / \mathrm{ml})$ : & $4.65 \mathrm{E}-01$ & $1.08 \mathrm{E}-01$ & Al \\
\hline & Uncorrected weight of impurity in sample $(\mu \mathrm{g})$ : & 64.17 & 13.82 & 77.99 \\
\hline & Weight of impurity in blank $(\mu \mathrm{g})$ : & 3.10 & 1.07 & \\
\hline & Minimum corrected weight of impurity in sample $(\mu \mathrm{g})$ : & 61.07 & 12.75 & 73.83 \\
\hline & Maximum corrected weight of impurity in sample $(\mu \mathrm{g})$ : & 61.07 & 12.75 & 73.83 \\
\hline \multirow{5}{*}{$\mathbf{T i}$} & Measured concentration of impurity in sample $(\mu \mathrm{g} / \mathrm{ml})$ : & $2.93 \mathrm{E}-02$ & $2.18 \mathrm{E}-02$ & $\mathrm{Ti}$ \\
\hline & Uncorrected weight of impurity in sample $(\mu \mathrm{g})$ : & 4.04 & 2.79 & 6.83 \\
\hline & Weight of impurity in blank $(\mu \mathrm{g})$ : & $<1.37$ & $<1.00$ & \\
\hline & Minimum corrected weight of impurity in sample $(\mu \mathrm{g})$ : & 2.68 & 1.79 & 4.47 \\
\hline & Maximum corrected weight of impurity in sample $(\mu q)$ : & 4.04 & 2.79 & 6.83 \\
\hline \multirow{5}{*}{$\mathbf{v}$} & Measured concentration of impurity in sample $(\mu \mathrm{g} / \mathrm{ml})$ : & $2.05 E-01$ & $3.33 \mathrm{E}-02$ & $\mathbf{v}$ \\
\hline & Uncorrected weight of impurity in sample $(\mu \mathrm{g})$ : & 28.29 & 4.26 & 32.55 \\
\hline & Weight of impurity in blank $(\mu \mathrm{g})$ : & $<0.34$ & $<0.25$ & \\
\hline & Minimum corrected weight of impurity in sample $(\mu \mathrm{g})$ : & 27.95 & 4.01 & 31.96 \\
\hline & Maximum corrected weight of impurity in sample $(\mu \mathrm{g})$ : & 28.29 & 4.26 & 32.55 \\
\hline
\end{tabular}

Comments

Data checked against RMAL. 2157 official results of analyses by FCM on 10/14/2009

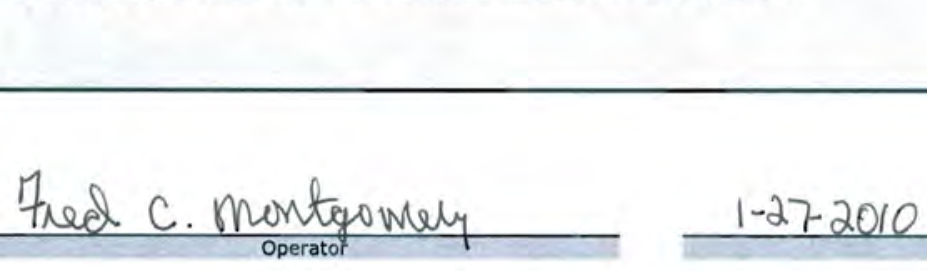


Data Report Form DRF-26A: Measurement of U Contamination or Impurities by Deconsolldation Leach

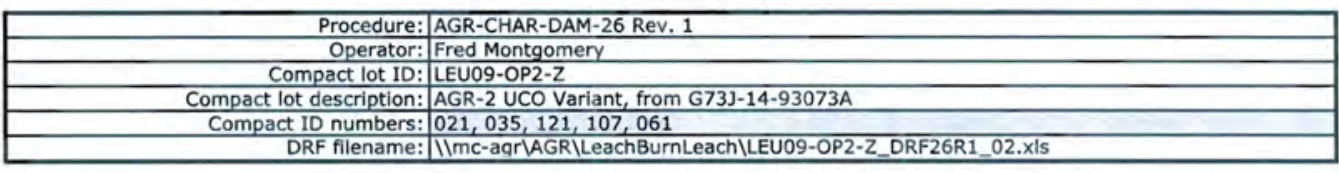

\begin{tabular}{|c|c|c|c|c|}
\hline & $\begin{array}{l}\text { Mean average weight uranium per particle }(g) \text { : } \\
\text { Uncertainty in mean averaqe weight uranium per particle }(q):\end{array}$ & & $\frac{3.96 E-04}{8.10 E-07}$ & \\
\hline & & First Leach & Second Leach & Total \\
\hline & Deconsolidation-leach solution ID: & $L 09081003$ & $L 09081203$ & \\
\hline & Number of compacts: & & & \\
\hline & Total volume of leach solution $(\mathrm{ml})$ : & 132.0 & 129.0 & \\
\hline & Radiochemical laboratory analysis number: & $2157-003$ & $2157-008$ & \\
\hline & Measured uranium concentration $(\mathrm{\mu g} / \mathrm{ml})$ : & $2.56 \mathrm{E}+00$ & $1.60 \mathrm{E}-01$ & \\
\hline & Uncertainty in uranium concentration $(\mathrm{\mu g} / \mathrm{ml})$ : & $2.56 \mathrm{E}-01$ & $1.60 \mathrm{E}-02$ & \\
\hline & Weight uranium leached $(9)$ : & $3.38 \mathrm{E}-04$ & $2.06 \mathrm{E}-05$ & $3.59 \mathrm{E}-04$ \\
\hline & Uncertainty in weight uranium leached (g): & $3.38 \mathrm{E}-05$ & $2.07 E-06$ & $3.39 \mathrm{E}-05$ \\
\hline & Effective number of exposed kernels: & 0.9 & 0.1 & 0.9 \\
\hline & Uncertainty in effective number of exposed kernels: & 0.1 & 0.0 & 0.1 \\
\hline & Measured concentration of impurity in sample $(\mu \mathrm{g} / \mathrm{ml})$ : & $<4.12 \mathrm{E}-02$ & $<4.12 \mathrm{E}-01$ & $\mathbf{F e}$ \\
\hline & Uncorrected weight of impurity in sample $(\mu \mathrm{g})$ : & $<5.44$ & $<53.15$ & $<58.59$ \\
\hline $\mathbf{F e}$ & Weight of impurity in blank $(\mu \mathrm{g})$ : & $<7.05$ & $<5.15$ & \\
\hline & Minimum corrected weight of impurity in sample $(\mu g)$ : & 0.00 & 0.00 & 0.00 \\
\hline & Maximum corrected weight of impurity in sample $(\mu \mathrm{g})$ : & 5.44 & 53.15 & 58.59 \\
\hline & Measured concentration of impurity in sample $(\mu \mathrm{g} / \mathrm{ml})$ : & $5.47 \mathrm{E}-03$ & $2.92 \mathrm{E}-03$ & $\mathrm{Cr}$ \\
\hline & Uncorrected weight of impurity in sample $(\mu \mathrm{g})$ : & 0.72 & 0.38 & 1.10 \\
\hline $\mathrm{Cr}$ & Weight of impurity in blank $(\mu \mathrm{g})$ : & $<0.34$ & $<0.25$ & \\
\hline . & Minimum corrected weight of impurity in sample $(\mu g)$ : & 0.38 & 0.13 & 0.51 \\
\hline & Maximum corrected weight of impurity in sample $(\mu \mathrm{g})$ : & 0.72 & 0.38 & 1.10 \\
\hline & Measured concentration of impurity in sample $(\mu \mathrm{g} / \mathrm{ml})$ : & $<1.91 \mathrm{E}-03$ & $<1.91 \mathrm{E}-03$ & $\mathrm{Mn}$ \\
\hline & Uncorrected weight of impurity in sample $(\mu \mathrm{g})$ : & $<0.25$ & $<0.25$ & $<0.50$ \\
\hline Mn & Weight of impurity in blank $(\mu \mathrm{g})$ : & $<0.33$ & $<0.24$ & \\
\hline & Minimum corrected weight of impurity in sample $(\mu g)$ : & 0.00 & 0.00 & 0.00 \\
\hline & Maximum corrected weight of impurity in sample $(\mu \mathrm{g})$ : & 0.25 & 0.25 & 0.50 \\
\hline & Measured concentration of impurity in sample $(\mathrm{\mu g} / \mathrm{ml})$ : & $<1.62 \mathrm{E}-03$ & $<1.62 \mathrm{E}-03$ & co \\
\hline & Uncorrected weight of Impurity in sample $(\mu \mathrm{g})$ : & $<0.21$ & $<0.21$ & $<0.42$ \\
\hline co & Weight of impurity in blank $(\mu \mathrm{g})$ : & $<0.28$ & $<0.20$ & \\
\hline & Minimum corrected weight of impurity in sample $(\mu \mathrm{g})$ : & 0.00 & 0.00 & 0.00 \\
\hline & Maximum corrected weight of impurity in sample $(\mu g)$ : & 0.21 & 0.21 & 0.42 \\
\hline & Measured concentration of impurity in sample $(\mu \mathrm{g} / \mathrm{ml})$ : & $1.09 \mathrm{E}-02$ & $<8.00 \mathrm{E}-03$ & $\mathrm{Ni}$ \\
\hline & Uncorrected weight of impurity in sample $(\mu \mathrm{g})$ : & 1.44 & $<1.03$ & $<2.47$ \\
\hline Ni & Weight of impurity in blank $(\mu \mathrm{g})$ : & $<1.37$ & $<1.00$ & \\
\hline & Minimum corrected weight of impurity in sample $(\mu \mathrm{g})$ : & 0.07 & 0.00 & 0.07 \\
\hline & Maximum corrected weight of impurity in sample $(\mu g)$ : & 1.44 & 1.03 & 2.47 \\
\hline & Measured concentration of impurity in sample $(\mu \mathrm{g} / \mathrm{ml})$ : & $1.27 E+00$ & $1.45 \mathrm{E}-01$ & $\mathbf{C a}$ \\
\hline & Uncorrected weight of impurity in sample $(\mu \mathrm{g})$ : & 167.64 & 18.71 & 186.35 \\
\hline $\mathbf{C a}$ & Weight of impurity in blank $(\mu \mathrm{g})$ : & 162.28 & 115.63 & \\
\hline & Minimum corrected weight of impurity in sample $(\mu g)$ : & 5.36 & 0.00 & 5.36 \\
\hline & Maximum corrected weight of impurity in sample $(\mu g)$ : & 5.36 & 0.00 & 5.36 \\
\hline & Measured concentration of impurity in sample $(\mathrm{\mu g} / \mathrm{ml})$ : & $4.06 \mathrm{E}-01$ & $5.06 \mathrm{E}-02$ & Al \\
\hline & Uncorrected weight of impurity in sample $(\mu \mathrm{g})$ : & 53.59 & 6.53 & 60.12 \\
\hline Al & Weight of impurity in blank $(\mu \mathrm{g})$ : & 3.10 & 1.07 & \\
\hline & Minimum corrected weight of impurity in sample $(\mu g)$ : & 50.50 & 5.46 & 55.95 \\
\hline & Maximum corrected weight of impurity in sample $(\mu \mathrm{g})$ : & 50.50 & 5.46 & 55.95 \\
\hline & Measured concentration of impurity in sample $(\mu \mathrm{g} / \mathrm{ml})$ : & $1.64 \mathrm{E}-02$ & $1.44 E-02$ & \\
\hline & Uncorrected weight of impurity in sample $(\mu \mathrm{g})$ : & 2.16 & 1.86 & 4.02 \\
\hline $\mathbf{T} \mathbf{i}$ & Weight of impurity in blank $(\mu \mathrm{g})$ : & $<1.37$ & $<1.00$ & \\
\hline & Minimum corrected weight of impurity in sample $(\mu \mathrm{g})$ : & 0.80 & 0.86 & 1.65 \\
\hline & Maximum corrected weight of impurity in sample $(\mu g)$ : & 2.16 & 1.86 & 4.02 \\
\hline & Measured concentration of impurity in sample $(\mu \mathrm{g} / \mathrm{ml})$ : & $1.98 \mathrm{E}-01$ & $4.43 \mathrm{E}-02$ & $\mathbf{v}$ \\
\hline & Uncorrected weight of impurity in sample $(\mu \mathrm{g})$ : & 26.14 & 5.71 & 31.85 \\
\hline $\mathbf{v}$ & Weight of impurity in blank $(\mu g)$ : & $<0.34$ & $<0.25$ & \\
\hline & Minimum corrected weight of impurity in sample $(\mu g)$ : & 25.79 & 5.46 & 31.26 \\
\hline & Maximum corrected weight of impurity in sample $(\mu g)$ : & 26.14 & 5.71 & 31.85 \\
\hline
\end{tabular}

\section{comments}

Data checked against RMAL 2157 official results of analyses by FCM on 10/14/2009

Fred c. Montgonney
$1-27-2010$ 


\begin{tabular}{|c|c|}
\hline Procedure: & AGR-CHAR-DAM-26 Rev. 1 \\
\hline Operator: & Fred Montgomery \\
\hline Compact lot ID: & LEU09-OP2-Z \\
\hline Compact lot description: & AGR-2 UCO Variant, from G73J-14-93073A \\
\hline Compact ID numbers: & $151,003,058,080,009$ \\
\hline DRF filename: & 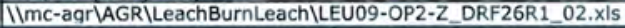 \\
\hline
\end{tabular}

\begin{tabular}{|c|c|c|c|c|}
\hline \multirow{2}{*}{\multicolumn{2}{|c|}{$\begin{array}{r}\text { Mean average weight uranium per particle }(g): \\
\text { Uncertainty in mean average weight uranium per particle }(q):\end{array}$}} & \multirow{2}{*}{\multicolumn{3}{|c|}{$\begin{array}{l}3.96 \mathrm{E}-04 \\
8.10 \mathrm{E}-07 \\
\end{array}$}} \\
\hline & & & & \\
\hline & & First Leach & Second Leach & Total \\
\hline & Deconsolidation-leach solution ID: & L09081005 & L09081205 & \\
\hline & Number of compacts: & & & \\
\hline & Total volume of leach solution $(\mathrm{ml})$ : & 133.0 & 130.0 & \\
\hline & & & & \\
\hline & Radiochemical laboratory analysis number: & $2157-005$ & $\frac{2157-010}{7215027}$ & \\
\hline & $\begin{array}{l}\text { Measured uranium concentration }(\mu \mathrm{g} / \mathrm{ml}) \text { : } \\
\text { Uncertainty in uranium concentration }(\mu \mathrm{g} / \mathrm{ml}) \text { : }\end{array}$ & $\frac{6.08 \mathrm{E}-02}{6.08 \mathrm{E}-03}$ & $\frac{7.31 E-03}{7.31 E-04}$ & \\
\hline & Weight uranium leached $(g):$ & $8.09 E-06$ & $9.50 \mathrm{E}-07$ & $9.04 E-06$ \\
\hline & Uncertainty in weight uranium leached $(g)$ : & $8.10 \mathrm{E}-07$ & $9.51 \mathrm{E}-08$ & $8.15 E-07$ \\
\hline & Effective number of exposed kernels: & 0.0 & 0.0 & 0.0 \\
\hline & Uncertainty in effective number of exposed kernels: & 0.0 & 0.0 & 0.0 \\
\hline \multirow{5}{*}{$\mathrm{Fe}$} & Measured concentration of impurity in sample $(\mu \mathrm{g} / \mathrm{ml})$ : & $<4.12 \mathrm{E}-02$ & $<4.12 \mathrm{E}-02$ & $\mathrm{Fe}$ \\
\hline & Uncorrected weight of impurity in sample $(\mu \mathrm{g})$ : & $<5.48$ & $<5.36$ & $<10.84$ \\
\hline & Weight of impurity in blank $(\mu \mathrm{g})$ : & $<7.05$ & $<5.15$ & \\
\hline & Minimum corrected weight of impurity in sample $(\mu \mathrm{g})$ : & 0.00 & 0.00 & 0.00 \\
\hline & Maximum corrected weight of impurity in sample $(\mu \mathrm{g})$ : & 5.48 & 5.36 & 10.84 \\
\hline \multirow{5}{*}{$\mathbf{C r}$} & Measured concentration of impurity in sample $(\mu \mathrm{g} / \mathrm{ml})$ : & 3.35E-03 & $<2.00 \mathrm{E}-03$ & $\mathrm{Cr}$ \\
\hline & Uncorrected weight of impurity in sample $(\mu \mathrm{g})$ : & 0.45 & $<0.26$ & $<0.71$ \\
\hline & Weight of impurity in blank $(\mu \mathrm{g})$ : & $<0.34$ & $<0.25$ & \\
\hline & Minimum corrected weight of impurity in sample $(\mu \mathrm{g})$ : & 0.10 & 0.00 & 0.10 \\
\hline & Maximum corrected weight of impurity in sample $(\mu \mathrm{g})$ : & 0.45 & 0.26 & 0.71 \\
\hline \multirow{5}{*}{ Mn } & Measured concentration of impurity in sample $(\mu \mathrm{g} / \mathrm{ml})$ : & $<1.91 \mathrm{E}-03$ & $<1.91 \mathrm{E}-03$ & Mn \\
\hline & Uncorrected weight of impurity in sample $(\mu \mathrm{g})$ : & $<0.25$ & $<0.25$ & $<0.50$ \\
\hline & Weight of impurity in blank $(\mu \mathrm{g})$ : & $<0.33$ & $<0.24$ & \\
\hline & Minimum corrected weight of impurity in sample $(\mu \mathrm{g})$ : & 0.00 & 0.00 & 0.00 \\
\hline & Maximum corrected weight of impurity in sample $(\mu \mathrm{g})$ : & 0.25 & 0.25 & 0.50 \\
\hline \multirow{5}{*}{ Co } & Measured concentration of impurity in sample $(\mu \mathrm{g} / \mathrm{ml})$ : & $<1.62 \mathrm{E}-03$ & $<1.62 \mathrm{E}-03$ & Co \\
\hline & Uncorrected weight of impurity in sample $(\mu \mathrm{g})$ : & $<0.22$ & $<0.21$ & $<0.43$ \\
\hline & Weight of impurity in blank $(\mu \mathrm{g})$ : & $<0.28$ & $<0.20$ & \\
\hline & Minimum corrected weight of impurity in sample $(\mu \mathrm{g})$ : & 0.00 & 0.00 & 0.00 \\
\hline & Maximum corrected weight of impurity in sample $(\mu \mathrm{g})$ : & 0.22 & 0.21 & 0.43 \\
\hline \multirow{5}{*}{$\mathbf{N i}$} & Measured concentration of impurity in sample $(\mu \mathrm{g} / \mathrm{ml})$ : & $<8.00 \mathrm{E}-03$ & $<8.00 \mathrm{E}-03$ & $\mathrm{Ni}$ \\
\hline & Uncorrected weight of impurity in sample $(\mu \mathrm{g})$ : & $<1.06$ & $<1.04$ & $<2.10$ \\
\hline & Weight of impurity in blank $(\mu \mathrm{g})$ : & $<1.37$ & $<1.00$ & \\
\hline & Minimum corrected weight of impurity in sample $(\mu \mathrm{g})$ : & 0.00 & 0.00 & 0.00 \\
\hline & Maximum corrected weight of impurity in sample $(\mu \mathrm{g})$ : & 1.06 & 1.04 & 2.10 \\
\hline \multirow{5}{*}{$\mathrm{Ca}$} & Measured concentration of impurity in sample $(\mu \mathrm{g} / \mathrm{ml})$ : & $3.28 \mathrm{E}+00$ & 4.09E-01 & $\mathbf{C a}$ \\
\hline & Uncorrected weight of impurity in sample $(\mu \mathrm{g})$ : & 436.24 & 53.17 & 489.41 \\
\hline & Weight of impurity in blank $(\mu g)$ : & 162.28 & 115.63 & \\
\hline & Minimum corrected weight of impurity in sample $(\mu \mathrm{g})$ : & 273.96 & 0.00 & 273.96 \\
\hline & Maximum corrected weight of impurity in sample $(\mu \mathrm{g})$ : & 273.96 & 0.00 & 273.96 \\
\hline \multirow{5}{*}{ Al } & Measured concentration of impurity in sample $(\mu \mathrm{g} / \mathrm{ml})$ : & 3.66E-01 & 5.35E-02 & Al \\
\hline & Uncorrected weight of impurity in sample $(\mu \mathrm{g})$ : & 48.68 & 6.96 & 55.63 \\
\hline & Weight of impurity in blank $(\mu \mathrm{g})$ : & 3.10 & 1.07 & \\
\hline & Minimum corrected weight of impurity in sample $(\mu \mathrm{g})$ : & 45.58 & 5.88 & 51.47 \\
\hline & Maximum corrected weight of impurity in sample $(\mu \mathrm{g})$ : & 45.58 & 5.88 & 51.47 \\
\hline \multirow{5}{*}{$\mathbf{T i}$} & Measured concentration of impurity in sample $(\mu \mathrm{g} / \mathrm{ml})$ : & $1.75 \mathrm{E}-02$ & $2.02 E-02$ & $\mathrm{Ti}$ \\
\hline & Uncorrected weight of impurity in sample $(\mu \mathrm{g})$ : & 2.33 & 2.63 & 4.95 \\
\hline & Weight of impurity in blank $(\mu \mathrm{g})$ : & $<1.37$ & $<1.00$ & \\
\hline & Minimum corrected weight of impurity in sample $(\mu \mathrm{g})$ : & 0.96 & 1.63 & 2.59 \\
\hline & Maximum corrected weight of impurity in sample $(\mu \mathrm{g})$ : & 2.33 & 2.63 & 4.95 \\
\hline \multirow{5}{*}{$\mathbf{v}$} & Measured concentration of impurity in sample $(\mu \mathrm{g} / \mathrm{ml})$ : & $1.72 \mathrm{E}-01$ & $5.56 \mathrm{E}-02$ & $\mathbf{v}$ \\
\hline & Uncorrected weight of impurity in sample $(\mu \mathrm{g})$ : & 22.88 & 7.23 & 30.10 \\
\hline & Weight of impurity in blank $(\mu \mathrm{g})$ : & $<0.34$ & $<0.25$ & \\
\hline & Minimum corrected weight of impurity in sample $(\mu \mathrm{g})$ : & 22.53 & 6.98 & 29.51 \\
\hline & Maximum corrected weight of impurity in sample $(\mu \mathrm{g})$ : & 22.88 & 7.23 & 30.10 \\
\hline
\end{tabular}

Comments

Data checked against RMAL 2157 official results of analyses by FCM on 10/14/2009

Fred c. Montgomeuy

$1-27-2010$

Operator 
Data Report Form DRF-26A: Measurement of U Contamination or Impuritles by Deconsolidation Leach

\begin{tabular}{|c|c|}
\hline Procedure: & AGR-CHAR-DAM- 26 Rev. 1 \\
\hline Operator: & Fred Montgomery \\
\hline Compact lot ID: & LEU09-OP2-Z \\
\hline Compact lot description: & AGR-2 UCO Variant, from G73J-14-93073A \\
\hline Compact ID numbers: & Deconsolidation Leach Blank \\
\hline DRF filename: & |Imc-agr|AGR\LeachBurnLeach|LEL \\
\hline
\end{tabular}

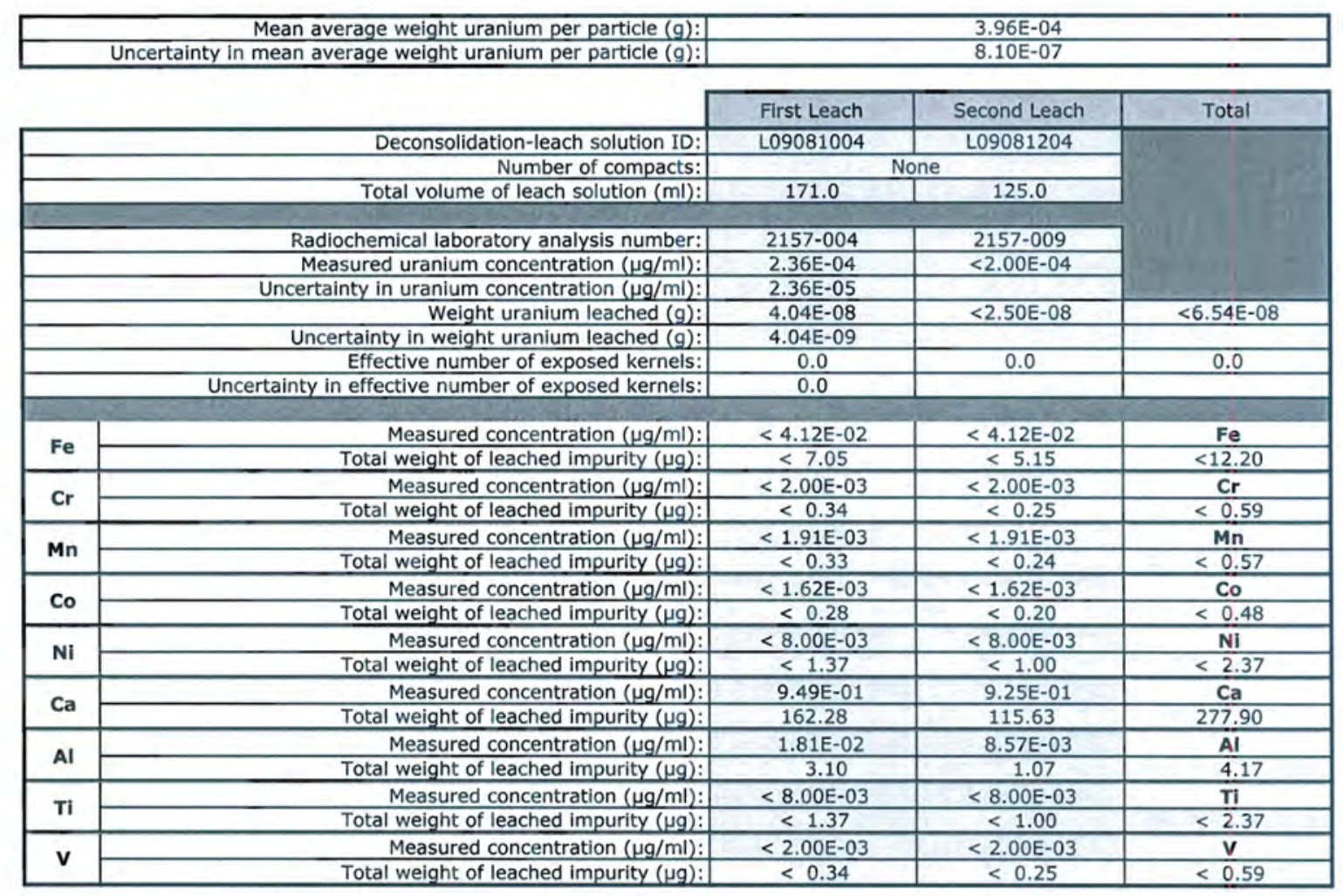

\section{Comments}

Data checked against RMAL 2157 official results of analyses by FCM on 10/14/2009 Fied C. Montgomey
$1-27-2010$ 


\begin{tabular}{|c|c|}
\hline Procedure: & AGR-CHAR-DAM-26 Rev. 1 \\
\hline Operator: & Fred Montgomery \\
\hline Compact lot ID: & LEU09-OP2-Z \\
\hline Compact lot description: & AGR-2 UCO Variant, from G73)-14-93073A \\
\hline Compact ID numbers: & $051,086,143,097,033$ \\
\hline DRF filename: & IImc-agr|AGRILeachBurnLeach|LEU09-OP2-Z_DRF26R1_02.xls \\
\hline
\end{tabular}

\begin{tabular}{|c|c|c|c|c|}
\hline \multirow{2}{*}{\multicolumn{2}{|c|}{$\begin{array}{r}\text { Mean average weight uranium per particle }(g): \\
\text { Uncertainty in mean averaqe weight uranium per particle }(q): \\
\end{array}$}} & \multicolumn{3}{|c|}{$3.96 \mathrm{E}-04$} \\
\hline & & \\
\hline & & First Leach & Second Leach & Total \\
\hline & Burn-leach solution ID: & B09082101 & B09082401 & \\
\hline & Number of compacts: & & & \\
\hline & Total volume of leach solution $(\mathrm{ml})$ : & 49.0 & 52.5 & \\
\hline & Radiochemical laboratory analysis number: & 2101001 & $2191-006$ & \\
\hline & $\begin{array}{c}\text { Radiochemical laboratory analysis number: } \\
\text { Measured uranium concentration }(\mu \mathrm{g} / \mathrm{ml}):\end{array}$ & $\frac{2191-001}{8.82 \mathrm{E}-01}$ & $\frac{2191-006}{5.21 \mathrm{E}-01}$ & \\
\hline & Uncertainty in uranium concentration $(\mu \mathrm{g} / \mathrm{ml})$ : & $8.82 \mathrm{E}-02$ & $5.21 E-02$ & \\
\hline & Weight uranium leached $(\mathrm{g})$ : & $4.32 \mathrm{E}-05$ & $2.74 \mathrm{E}-05$ & $7.06 \mathrm{E}-05$ \\
\hline & Uncertainty in weight uranium leached $(\mathrm{g})$ : & $4.36 \mathrm{E}-06$ & $2.76 \mathrm{E}-06$ & $5.16 \mathrm{E}-06$ \\
\hline & Number of leached kernels: & 0.1 & 0.1 & 0.2 \\
\hline & Uncertainty in number of leached kernels: & 0.0 & 0.0 & 0.0 \\
\hline \multirow{5}{*}{$\mathrm{Fe}$} & Measured concentration of impurity in sample $(\mu \mathrm{g} / \mathrm{ml})$ : & $6.59 \mathrm{E}-02$ & $<4.12 \mathrm{E}-02$ & $\mathrm{Fe}$ \\
\hline & Uncorrected weight of impurity in sample $(\mu \mathrm{g})$ : & 3.23 & $<2.16$ & $<5.39$ \\
\hline & Weight of impurity in blank $(\mu \mathrm{g})$ : & $<2.12$ & $<2.13$ & \\
\hline & Minimum corrected weight of impurity in sample $(\mu \mathrm{g})$ : & 1.11 & 0.00 & 1.11 \\
\hline & Maximum corrected weight of impurity in sample $(\mu \mathrm{g})$ : & 3.23 & 2.16 & 5.39 \\
\hline \multirow{5}{*}{$\mathbf{C r}$} & Measured concentration of impurity in sample $(\mu \mathrm{g} / \mathrm{ml})$ : & $2.33 \mathrm{E}-02$ & $<2.00 \mathrm{E}-03$ & $\mathrm{Cr}$ \\
\hline & Uncorrected weight of Impurity in sample $(\mu \mathrm{g})$ : & 1.14 & $<0.11$ & $<1.25$ \\
\hline & Weight of impurity in blank $(\mu \mathrm{g})$ : & $<0.10$ & $<0.10$ & \\
\hline & Minimum corrected weight of impurity in sample $(\mu \mathrm{g})$ : & 1.04 & 0.00 & 1.04 \\
\hline & Maximum corrected weight of impurity in sample $(\mu \mathrm{g})$ : & 1.14 & 0.11 & 1.25 \\
\hline \multirow{5}{*}{ Mn } & Measured concentration of impurity in sample $(\mu \mathrm{g} / \mathrm{ml})$ : & $<1.91 \mathrm{E}-03$ & $<1.91 \mathrm{E}-03$ & Mn \\
\hline & Uncorrected weight of impurity in sample $(\mu \mathrm{g})$ : & $<0.09$ & $<0.10$ & $<0.19$ \\
\hline & Weight of impurity in blank $(\mu \mathrm{g})$ : & $<0.10$ & $<0.10$ & \\
\hline & Minimum corrected weight of impurity in sample $(\mu g)$ : & 0.00 & 0.00 & 0.00 \\
\hline & Maximum corrected weight of impurity in sample $(\mu \mathrm{g})$ : & 0.09 & 0.10 & 0.19 \\
\hline \multirow{5}{*}{ Co } & Measured concentration of impurity in sample $(\mu \mathrm{g} / \mathrm{ml})$ : & $<1.62 \mathrm{E}-03$ & $<1.62 \mathrm{E}-03$ & $\mathrm{Co}$ \\
\hline & Uncorrected weight of impurity in sample $(\mu \mathrm{g})$ : & $<0.08$ & $<0.09$ & $<0.16$ \\
\hline & Weight of impurity in blank $(\mu \mathrm{g})$ : & $<0.08$ & $<0.08$ & +2 \\
\hline & Minimum corrected weight of impurity in sample $(\mu \mathrm{g})$ : & 0.00 & 0.00 & 0.00 \\
\hline & Maximum corrected weight of impurity in sample $(\mu \mathrm{g})$ : & 0.08 & 0.09 & 0.16 \\
\hline \multirow{5}{*}{$\mathrm{Ni}$} & Measured concentration of impurity in sample $(\mu \mathrm{g} / \mathrm{ml})$ : & $5.19 \mathrm{E}-02$ & $<8.00 \mathrm{E}-03$ & $\mathrm{Ni}$ \\
\hline & Uncorrected weight of impurity in sample $(\mu \mathrm{g})$ : & 2.54 & $<0.42$ & $<2.96$ \\
\hline & Weight of impurity in blank $(\mu \mathrm{g})$ : & $<0.41$ & $<0.41$ & \\
\hline & Minimum corrected weight of impurity in sample $(\mu \mathrm{g})$ : & 2.13 & 0.00 & 2.13 \\
\hline & Maximum corrected weight of impurity in sample $(\mu \mathrm{g})$ : & 2.54 & 0.42 & 2.96 \\
\hline \multirow{5}{*}{$\mathrm{Ca}$} & Measured concentration of impurity in sample $(\mu \mathrm{g} / \mathrm{ml})$ : & $2.18 E+00$ & $4.56 \mathrm{E}-01$ & $\mathrm{Ca}$ \\
\hline & Uncorrected weight of impurity in sample $(\mu \mathrm{g})$ : & 106.82 & 23.94 & 130.76 \\
\hline & Weight of impurity in blank $(\mu \mathrm{g})$ : & 11.33 & $<5.18$ & \\
\hline & Minimum corrected weight of impurity in sample $(\mu \mathrm{g})$ : & 95.49 & 18.76 & 114.25 \\
\hline & Maximum corrected weight of impurity in sample $(\mu g)$ : & 95.49 & 23.94 & 119.43 \\
\hline \multirow{5}{*}{ Al } & Measured concentration of impurity in sample $(\mu \mathrm{g} / \mathrm{ml})$ : & $1.70 E+00$ & $4.80 \mathrm{E}-02$ & Al \\
\hline & Uncorrected weight of impurity in sample $(\mu \mathrm{g})$ : & 83.30 & 2.52 & 85.82 \\
\hline & Weight of impurity in blank $(\mu \mathrm{g})$ : & 2.80 & 0.60 & \\
\hline & Minimum corrected weight of impurity in sample $(\mu \mathrm{g})$ : & 80.50 & 1.92 & 82.43 \\
\hline & Maximum corrected weight of impurity in sample $(\mu \mathrm{g})$ : & 80.50 & 1.92 & 82.43 \\
\hline \multirow{5}{*}{$\mathbf{T i}$} & Measured concentration of impurity in sample $(\mu \mathrm{g} / \mathrm{ml})$ : & $1.60 \mathrm{E}-01$ & $<8.00 \mathrm{E}-03$ & $\mathrm{Ti}$ \\
\hline & Uncorrected weight of impurity in sample $(\mu \mathrm{g})$ : & 7.84 & $<0.42$ & $<8.26$ \\
\hline & Weight of impurity in blank $(\mu \mathrm{g})$ : & $<0.41$ & $<0.41$ & \\
\hline & Minimum corrected weight of impurity in sample $(\mu \mathrm{g})$ : & 7.43 & 0.00 & 7.43 \\
\hline & Maximum corrected weight of impurity in sample $(\mu \mathrm{g})$ : & 7.84 & 0.42 & 8.26 \\
\hline \multirow{5}{*}{$\mathbf{v}$} & Measured concentration of impurity in sample $(\mu \mathrm{g} / \mathrm{ml})$ : & $1.18 \mathrm{E}+00$ & 1.07E-02 & $\mathbf{V}$ \\
\hline & Uncorrected weight of impurity in sample $(\mu \mathrm{g})$ : & 57.82 & 0.56 & 58.38 \\
\hline & Weight of impurity in blank $(\mu \mathrm{g})$ : & $<0.10$ & $<0.10$ & \\
\hline & Minimum corrected weight of impurity in sample $(\mu \mathrm{g})$ : & 57.72 & 0.46 & 58.18 \\
\hline & Maximum corrected weight of impurity in sample $(\mu \mathrm{g})$ : & 57.82 & 0.56 & 58.38 \\
\hline
\end{tabular}

Comments

\begin{tabular}{|l|l|}
\hline Data checked against RMAL 2191 official results of analyses by FCM on $10 / 14 / 2009$ \\
\hline
\end{tabular}

Freel c. Montgomey
$1-27-2010$ 


\begin{tabular}{|c|c|}
\hline Procedure: & AGR-CHAR-DAM-26 Rev. 1 \\
\hline Operator: & Fred Montgomery \\
\hline Compact lot ID: & LEU09-OP2-Z \\
\hline Compact lot description: & AGR-2 UCO Variant, from G73]-14-93073A \\
\hline Compact ID numbers: & $030,024,090,013,010$ \\
\hline DRF filename: & 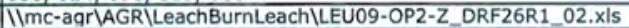 \\
\hline
\end{tabular}

\begin{tabular}{|c|c|c|c|c|}
\hline \multirow{2}{*}{\multicolumn{2}{|c|}{\begin{tabular}{r|} 
Mean average weight uranium per particle $(g):$ \\
Uncertainty in mean average weight uranium per particle $(\mathrm{q}):$ \\
\end{tabular}}} & \multirow{2}{*}{\multicolumn{3}{|c|}{$\frac{3.96 \mathrm{E}-04}{8.10 \mathrm{E}-07}$}} \\
\hline & & & & \\
\hline & & First Leach & Second Leach & Total \\
\hline \multicolumn{2}{|r|}{ Burn-leach solution ID: } & $B 09082102$ & B09082402 & \\
\hline & Number of compacts: & \multicolumn{2}{|c|}{5} & \\
\hline \multirow{2}{*}{\multicolumn{2}{|c|}{ Total volume of leach solution $(\mathrm{ml}):$}} & 49.8 & 51.8 & \\
\hline & & 7 & 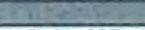 & \\
\hline \multicolumn{2}{|r|}{ Radiochemical laboratory analysis number: } & 2191-002 & 2191-007 & \\
\hline \multirow{2}{*}{\multicolumn{2}{|c|}{$\begin{array}{l}\text { Measured uranium concentration }(\mu \mathrm{g} / \mathrm{ml}) \text { : } \\
\text { Uncertainty in uranium concentration }(\mu \mathrm{g} / \mathrm{ml}) \text { : }\end{array}$}} & $3.32 \mathrm{E}-01$ & $4.37 E-03$ & \\
\hline & & $3.32 \mathrm{E}-02$ & $4.37 E-04$ & \\
\hline \multirow{2}{*}{\multicolumn{2}{|c|}{$\begin{array}{l}\text { Weight uranium leached }(\mathrm{g}) \text { : } \\
\text { Uncertainty in weight uranium leached }(\mathrm{g}) \text { : }\end{array}$}} & $1.65 \mathrm{E}-05$ & $2.26 \mathrm{E}-07$ & $1.68 \mathrm{E}-05$ \\
\hline & & $1.67 E-06$ & $2.28 \mathrm{E}-08$ & $1.67 \mathrm{E}-06$ \\
\hline \multirow{2}{*}{\multicolumn{2}{|c|}{$\begin{array}{r}\text { Number of leached kernels: } \\
\text { Uncertainty in number of leached kernels: }\end{array}$}} & 0.0 & 0.0 & 0.0 \\
\hline & & 0.0 & 0.0 & 0.0 \\
\hline \multirow{5}{*}{$\mathbf{F e}$} & Measured concentration of impurity in sample $(\mu \mathrm{g} / \mathrm{ml})$ : & $1.00 \mathrm{E}-01$ & $<4.12 \mathrm{E}-02$ & $\mathrm{Fe}$ \\
\hline & Uncorrected weight of impurity in sample $(\mu \mathrm{g})$ : & 4.98 & $<2.13$ & $<7.11$ \\
\hline & Weight of impurity in blank $(\mu \mathrm{g})$ : & $<2.12$ & $<2.13$ & \\
\hline & Minimum corrected weight of impurity in sample $(\mu \mathrm{g})$ : & 2.86 & 0.00 & 2.86 \\
\hline & Maximum corrected weight of impurity in sample $(\mu \mathrm{g})$ : & 4.98 & 2.13 & 7.11 \\
\hline \multirow{5}{*}{$\mathbf{C r}$} & Measured concentration of impurity in sample $(\mu \mathrm{g} / \mathrm{ml})$ : & $2.38 \mathrm{E}-02$ & $<2.00 \mathrm{E}-03$ & $\mathrm{Cr}$ \\
\hline & Uncorrected weight of impurity in sample $(\mu \mathrm{g})$ : & 1.19 & $<0.10$ & $<1.29$ \\
\hline & Weight of impurity in blank $(\mu \mathrm{g})$ : & $<0.10$ & $<0.10$ & \\
\hline & Minimum corrected weight of impurity in sample $(\mu \mathrm{g})$ : & 1.08 & 0.00 & 1.08 \\
\hline & Maximum corrected weight of impurity in sample $(\mu \mathrm{g})$ : & 1.19 & 0.10 & 1.29 \\
\hline \multirow{5}{*}{ Mn } & Measured concentration of impurity in sample $(\mu \mathrm{g} / \mathrm{ml})$ : & $<1.91 \mathrm{E}-03$ & $<1.91 \mathrm{E}-03$ & Mn \\
\hline & Uncorrected weight of impurity in sample $(\mu \mathrm{g})$ : & $<0.10$ & $<0.10$ & $<0.19$ \\
\hline & Weight of impurity in blank $(\mu \mathrm{g})$ : & $<0.10$ & $<0.10$ & +2 \\
\hline & Minimum corrected weight of impurity in sample $(\mu \mathrm{g})$ : & 0.00 & 0.00 & 0.00 \\
\hline & Maximum corrected weight of impurity in sample $(\mu \mathrm{g})$ : & 0.10 & 0.10 & 0.19 \\
\hline \multirow{5}{*}{ Co } & Measured concentration of impurity in sample $(\mu \mathrm{g} / \mathrm{ml})$ : & $<1.62 \mathrm{E}-03$ & $<1.62 \mathrm{E}-03$ & Co \\
\hline & Uncorrected weight of impurity in sample $(\mu \mathrm{g})$ : & $<0.08$ & $<0.08$ & $<0.16$ \\
\hline & Weight of impurity in blank $(\mu \mathrm{g})$ : & $<0.08$ & $<0.08$ & \\
\hline & Minimum corrected weight of impurity in sample $(\mu g)$ : & 0.00 & 0.00 & 0.00 \\
\hline & Maximum corrected weight of impurity in sample $(\mu \mathrm{g})$ : & 0.08 & 0.08 & 0.16 \\
\hline \multirow{5}{*}{$\mathbf{N i}$} & Measured concentration of impurity in sample $(\mu \mathrm{g} / \mathrm{ml})$ : & $4.21 \mathrm{E}-02$ & $<8.00 \mathrm{E}-03$ & $\mathrm{Ni}$ \\
\hline & Uncorrected weight of impurity in sample $(\mu \mathrm{g})$ : & 2.10 & $<0.41$ & $<2.51$ \\
\hline & Weight of impurity in blank $(\mu \mathrm{g})$ : & $<0.41$ & $<0.41$ & \\
\hline & Minimum corrected weight of impurity in sample $(\mu \mathrm{g})$ : & 1.68 & 0.00 & 1.68 \\
\hline & Maximum corrected weight of impurity in sample $(\mu \mathrm{g})$ : & 2.10 & 0.41 & 2.51 \\
\hline \multirow{5}{*}{$\mathbf{C a}$} & Measured concentration of impurity in sample $(\mu \mathrm{g} / \mathrm{ml})$ : & $2.05 E+00$ & $1.42 \mathrm{E}-01$ & $\mathrm{Ca}$ \\
\hline & Uncorrected weight of impurity in sample $(\mu \mathrm{g})$ : & 102.09 & 7.36 & 109.45 \\
\hline & Weight of impurity in blank $(\mu \mathrm{g})$ : & 11.33 & $<5.18$ & \\
\hline & Minimum corrected weight of impurity in sample $(\mu \mathrm{g})$ : & 90.76 & 2.18 & 92.94 \\
\hline & Maximum corrected weight of impurity in sample $(\mu \mathrm{g})$ : & 90.76 & 7.36 & 98.12 \\
\hline \multirow{5}{*}{ Al } & Measured concentration of impurity in sample $(\mu \mathrm{g} / \mathrm{ml})$ : & $1.83 E+00$ & $5.05 E-02$ & Al \\
\hline & Uncorrected weight of impurity in sample $(\mu \mathrm{g})$ : & 91.13 & 2.62 & 93.75 \\
\hline & Weight of impurity in blank $(\mu \mathrm{g})$ : & 2.80 & 0.60 & \\
\hline & Minimum corrected weight of impurity in sample $(\mu \mathrm{g})$ : & 88.34 & 2.02 & 90.36 \\
\hline & Maximum corrected weight of impurity in sample $(\mu \mathrm{g})$ : & 88.34 & 2.02 & 90.36 \\
\hline \multirow{5}{*}{$\mathbf{T i}$} & Measured concentration of impurity in sample $(\mu \mathrm{g} / \mathrm{ml})$ : & $1.42 \mathrm{E}-01$ & $<8.00 \mathrm{E}-03$ & $\mathrm{Ti}$ \\
\hline & Uncorrected weight of impurity in sample $(\mu \mathrm{g})$ : & 7.07 & $<0.41$ & $<7.49$ \\
\hline & Weight of impurity in blank $(\mu \mathrm{g})$ : & $<0.41$ & $<0.41$ & \\
\hline & Minimum corrected weight of impurity in sample $(\mu \mathrm{g})$ : & 6.66 & 0.00 & 6.66 \\
\hline & Maximum corrected weight of impurity in sample $(\mu g)$ : & 7.07 & 0.41 & 7.49 \\
\hline & Measured concentration of impurity in sample $(\mu \mathrm{g} / \mathrm{ml})$ : & $2.27 E-01$ & 7.07E-03 & $\mathbf{v}$ \\
\hline & Uncorrected weight of impurity in sample $(\mu \mathrm{g})$ : & 11.30 & 0.37 & 11.67 \\
\hline $\mathbf{v}$ & Weight of impurity in blank $(\mu \mathrm{g})$ : & $<0.10$ & $<0.10$ & \\
\hline & Minimum corrected weight of impurity in sample $(\mu g)$ : & 11.20 & 0.26 & 11.46 \\
\hline & Maximum corrected weight of impurity in sample $(\mu \mathrm{g})$ : & 11.30 & 0.37 & 11.67 \\
\hline
\end{tabular}

Comments

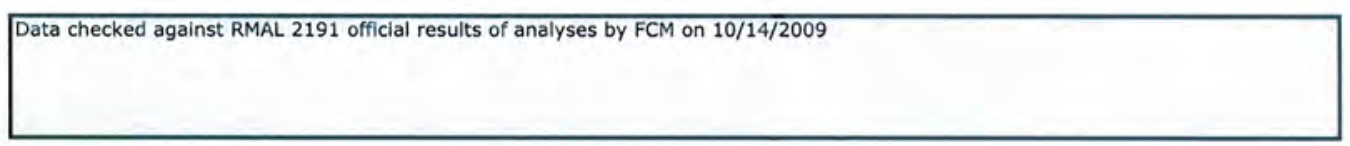

\section{Fied c. Montgomery $-1-27-2010$}

Thed c. Moprato 


\begin{tabular}{|c|c|}
\hline Procedure: & AGR-CHAR-DAM-26 Rev. 1 \\
\hline Operator: & Fred Montgomery \\
\hline Compact lot ID: & LEU09-OP2-Z \\
\hline Compact lot description: & AGR-2 UCO Variant, from G73J-14-93073A \\
\hline Compact ID numbers: & $021,035,121,107,061$ \\
\hline DRF filename: & IImc-agr|AGR\LeachBurnLeach|LEU09-OP2-Z_DRF26R1_02.xIs \\
\hline
\end{tabular}

\begin{tabular}{|c|c|c|c|c|}
\hline \multirow{2}{*}{\multicolumn{2}{|c|}{$\begin{array}{r}\text { Mean average weight uranium per particle }(g): \\
\text { Uncertainty in mean average weight uranium per particle }(q):\end{array}$}} & \multirow{2}{*}{\multicolumn{3}{|c|}{$\begin{array}{l}3.96 \mathrm{E}-04 \\
8.10 \mathrm{E}-07\end{array}$}} \\
\hline & & & & \\
\hline & & First Leach & Second Leach & Total \\
\hline \multicolumn{2}{|r|}{ Burn-leach solution ID: } & $B 09082103$ & B09082403 & \\
\hline & Number of compacts: & \multicolumn{2}{|c|}{5} & \\
\hline \multirow{2}{*}{\multicolumn{2}{|c|}{ Total volume of leach solution $(\mathrm{ml})$ : }} & 50.0 & 50.0 & \\
\hline & & & & \\
\hline \multirow{2}{*}{\multicolumn{2}{|c|}{ Radiochemical laboratory analysis number: }} & 2191-003 & $2191-008$ & \\
\hline & & $3.56 \mathrm{E}-01$ & $2.86 \mathrm{E}-01$ & \\
\hline \multicolumn{2}{|r|}{ Uncertainty in uranium concentration $(\mu \mathrm{g} / \mathrm{ml})$ : } & $3.56 \mathrm{E}-02$ & $2.86 \mathrm{E}-02$ & \\
\hline \multirow{2}{*}{\multicolumn{2}{|c|}{$\begin{array}{l}\text { Weight uranium leached }(g) \text { : } \\
\text { Uncertainty in weight uranium leached }(g) \text { : }\end{array}$}} & $1.78 \mathrm{E}-05$ & $1.43 \mathrm{E}-05$ & $3.21 \mathrm{E}-05$ \\
\hline & & $1.79 \mathrm{E}-06$ & $1.44 \mathrm{E}-06$ & $2.30 \mathrm{E}-06$ \\
\hline \multirow{2}{*}{\multicolumn{2}{|c|}{$\begin{array}{r}\text { Number of leached kernels: } \\
\text { Uncertainty in number of leached kernels: } \\
\end{array}$}} & 0.0 & 0.0 & 0.1 \\
\hline & & 0.0 & 0.0 & 0.0 \\
\hline \multirow{5}{*}{$\mathrm{Fe}$} & Measured concentration of impurity in sample $(\mu \mathrm{g} / \mathrm{ml})$ : & $6.32 \mathrm{E}-02$ & $<4.12 \mathrm{E}-02$ & $\mathbf{F e}$ \\
\hline & Uncorrected weight of impurity in sample $(\mu \mathrm{g})$ : & 3.16 & $<2.06$ & $<5.22$ \\
\hline & Weight of impurity in blank $(\mu g)$ : & $<2.12$ & $<2.13$ & \\
\hline & Minimum corrected weight of impurity in sample $(\mu \mathrm{g})$ : & 1.04 & 0.00 & 1.04 \\
\hline & Maximum corrected weight of impurity in sample $(\mu \mathrm{g})$ : & 3.16 & 2.06 & 5.22 \\
\hline \multirow{5}{*}{$\mathrm{Cr}$} & Measured concentration of impurity in sample $(\mu \mathrm{g} / \mathrm{ml})$ : & $2.15 E-02$ & $<2.00 \mathrm{E}-03$ & $\mathrm{Cr}$ \\
\hline & Uncorrected weight of impurity in sample $(\mu \mathrm{g})$ : & 1.08 & $<0.10$ & $<1.18$ \\
\hline & Weight of impurity in blank $(\mu \mathrm{g})$ : & $<0.10$ & $<0.10$ & \\
\hline & Minimum corrected weight of impurity in sample $(\mu g)$ : & 0.97 & 0.00 & 0.97 \\
\hline & Maximum corrected weight of impurity in sample $(\mu \mathrm{g})$ : & 1.08 & 0.10 & 1.18 \\
\hline \multirow{5}{*}{ Mn } & Measured concentration of impurity in sample $(\mu \mathrm{g} / \mathrm{ml})$ : & $<1.91 \mathrm{E}-03$ & $<1.91 \mathrm{E}-03$ & Mn \\
\hline & Uncorrected weight of impurity in sample $(\mu \mathrm{g})$ : & $<0.10$ & $<0.10$ & $<0.19$ \\
\hline & Weight of impurity in blank $(\mu \mathrm{g})$ : & $<0.10$ & $<0.10$ & \\
\hline & Minimum corrected weight of impurity in sample $(\mu \mathrm{g})$ : & 0.00 & 0.00 & 0.00 \\
\hline & Maximum corrected weight of impurity in sample $(\mu \mathrm{g})$ : & 0.10 & 0.10 & 0.19 \\
\hline \multirow{5}{*}{ Co } & Measured concentration of impurity in sample $(\mu \mathrm{g} / \mathrm{ml})$ : & $<1.62 \mathrm{E}-03$ & $<1.62 \mathrm{E}-03$ & Co \\
\hline & Uncorrected weight of Impurity in sample $(\mu \mathrm{g})$ : & $<0.08$ & $<0.08$ & $<0.16$ \\
\hline & Weight of impurity in blank $(\mu \mathrm{g})$ : & $<0.08$ & $<0.08$ & \\
\hline & Minimum corrected weight of impurity in sample $(\mu \mathrm{g})$ : & 0.00 & 0.00 & 0.00 \\
\hline & Maximum corrected weight of impurity in sample $(\mu \mathrm{g})$ : & 0.08 & 0.08 & 0.16 \\
\hline \multirow{5}{*}{$\mathbf{N i}$} & Measured concentration of impurity in sample $(\mu \mathrm{g} / \mathrm{ml})$ : & $5.54 \mathrm{E}-02$ & $<8.00 \mathrm{E}-03$ & $\mathrm{Ni}$ \\
\hline & Uncorrected weight of impurity in sample $(\mu \mathrm{g})$ : & 2.77 & $<0.40$ & $<3.17$ \\
\hline & Weight of impurity in blank $(\mu \mathrm{g})$ : & $<0.41$ & $<0.41$ & \\
\hline & Minimum corrected weight of impurity in sample $(\mu \mathrm{g})$ : & 2.36 & 0.00 & 2.36 \\
\hline & Maximum corrected weight of impurity in sample $(\mu \mathrm{g})$ : & 2.77 & 0.40 & 3.17 \\
\hline \multirow{5}{*}{$\mathrm{Ca}$} & Measured concentration of impurity in sample $(\mu \mathrm{g} / \mathrm{ml})$ : & $2.00 E+00$ & $1.25 \mathrm{E}-01$ & $\mathbf{C a}$ \\
\hline & Uncorrected weight of impurity in sample $(\mu \mathrm{g})$ : & 100.00 & 6.25 & 106.25 \\
\hline & Weight of impurity in blank $(\mu g)$ : & 11.33 & $<5.18$ & \\
\hline & Minimum corrected weight of impurity in sample $(\mu \mathrm{g})$ : & 88.67 & 1.07 & 89.74 \\
\hline & Maximum corrected weight of impurity in sample $(\mu g)$ : & 88.67 & 6.25 & 94.92 \\
\hline \multirow{5}{*}{ Al } & Measured concentration of impurity in sample $(\mu \mathrm{g} / \mathrm{ml})$ : & $1.63 E+00$ & 7.89E-02 & Al \\
\hline & Uncorrected weight of impurity in sample $(\mu \mathrm{g})$ : & 81.50 & 3.95 & 85.45 \\
\hline & Weight of impurity in blank $(\mu \mathrm{g})$ : & 2.80 & 0.60 & \\
\hline & Minimum corrected weight of impurity in sample $(\mu \mathrm{g})$ : & 78.70 & 3.35 & 82.05 \\
\hline & Maximum corrected weight of impurity in sample $(\mu \mathrm{g})$ : & 78.70 & 3.35 & 82.05 \\
\hline \multirow{5}{*}{$\mathbf{T i}$} & Measured concentration of impurity in sample $(\mu \mathrm{g} / \mathrm{ml})$ : & $1.70 \mathrm{E}-01$ & $1.22 \mathrm{E}-02$ & $\mathrm{Ti}$ \\
\hline & Uncorrected weight of impurity in sample $(\mu \mathrm{g})$ : & 8.50 & 0.61 & 9.11 \\
\hline & Weight of impurity in blank $(\mu \mathrm{g})$ : & $<0.41$ & $<0.41$ & \\
\hline & Minimum corrected weight of impurity in sample $(\mu \mathrm{g})$ : & 8.09 & 0.20 & 8.28 \\
\hline & Maximum corrected weight of impurity in sample $(\mu \mathrm{g})$ : & 8.50 & 0.61 & 9.11 \\
\hline & Measured concentration of impurity in sample $(\mu \mathrm{g} / \mathrm{ml})$ : & $1.14 \mathrm{E}+00$ & 1.17E-02 & $\mathbf{V}$ \\
\hline & Uncorrected weight of impurity in sample $(\mu \mathrm{g})$ : & 57.00 & 0.59 & 57.59 \\
\hline $\mathbf{v}$ & Weight of impurity in blank $(\mu \mathrm{g})$ : & $<0.10$ & $<0.10$ & \\
\hline & Minimum corrected weight of impurity in sample $(\mu \mathrm{g})$ : & 56.90 & 0.48 & 57.38 \\
\hline & Maximum corrected weight of impurity in sample $(\mu \mathrm{g})$ : & 57.00 & 0.59 & 57.59 \\
\hline
\end{tabular}

Comments

\begin{tabular}{|l|l|} 
Data checked against RMAL 2191 official results of analyses by FCM on $10 / 14 / 2009$ \\
\hline
\end{tabular}

\section{Feed c. montgomely $1-27-2010$}

operator Date 
Data Report Form DRF-26B: Measurement of SiC Burn-Leach Defects or Impurities by Burn-Leach

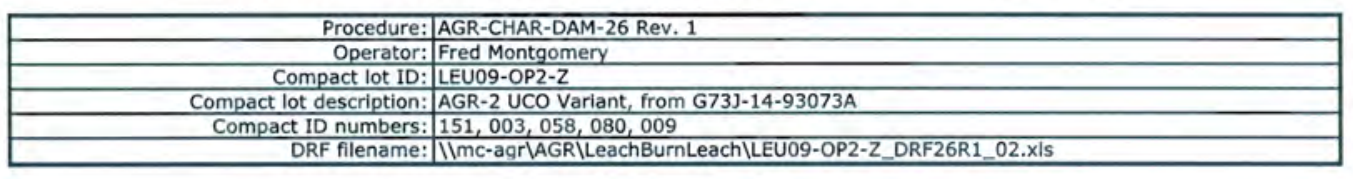

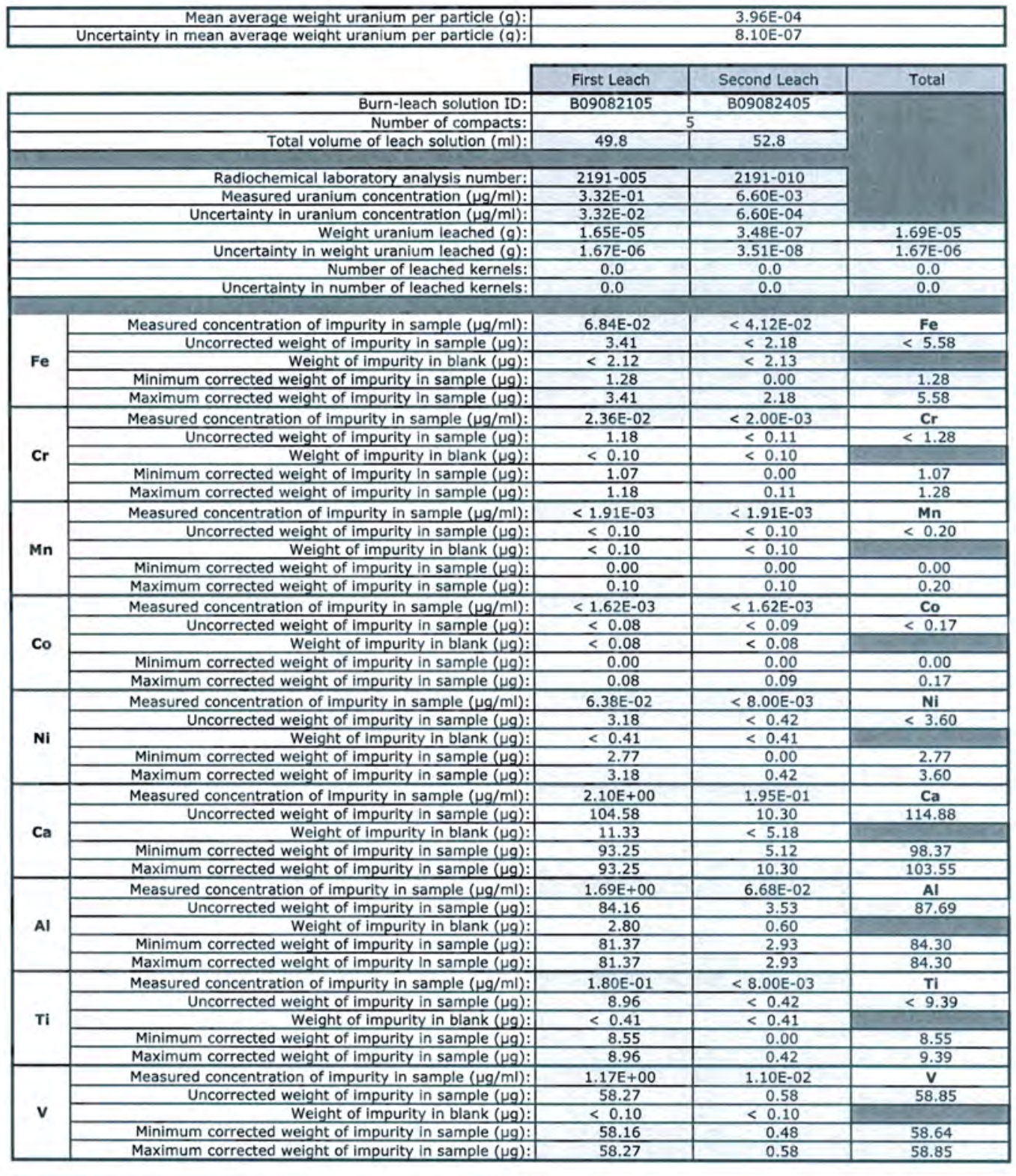

Comments

Data checked against RMAL 2191 official results of analyses by FCM on 10/14/2009

Tred c. Montgomery $\frac{1-27-2010}{\text { operator }}$ 
Data Report Form DRF-26B: Measurement of SiC Burn-Leach Defects or Impurities by Burn-Leach

\begin{tabular}{|c|c|}
\hline Procedure: & AGR-CHAR-DAM-26 Rev. 1 \\
\hline Operator: & Fred Montgomery \\
\hline Compact lot ID: & LEU09-OP2-Z \\
\hline Compact lot description: & AGR-2 UCO Variant, from G73]-14-93073A \\
\hline Compact ID numbers: & Burn-Leach Blank \\
\hline DRF filename: & IImc-agr\AGR\LeachBurnLeach\LEU09-OP2-Z_DRF26R1_02.xIs \\
\hline
\end{tabular}

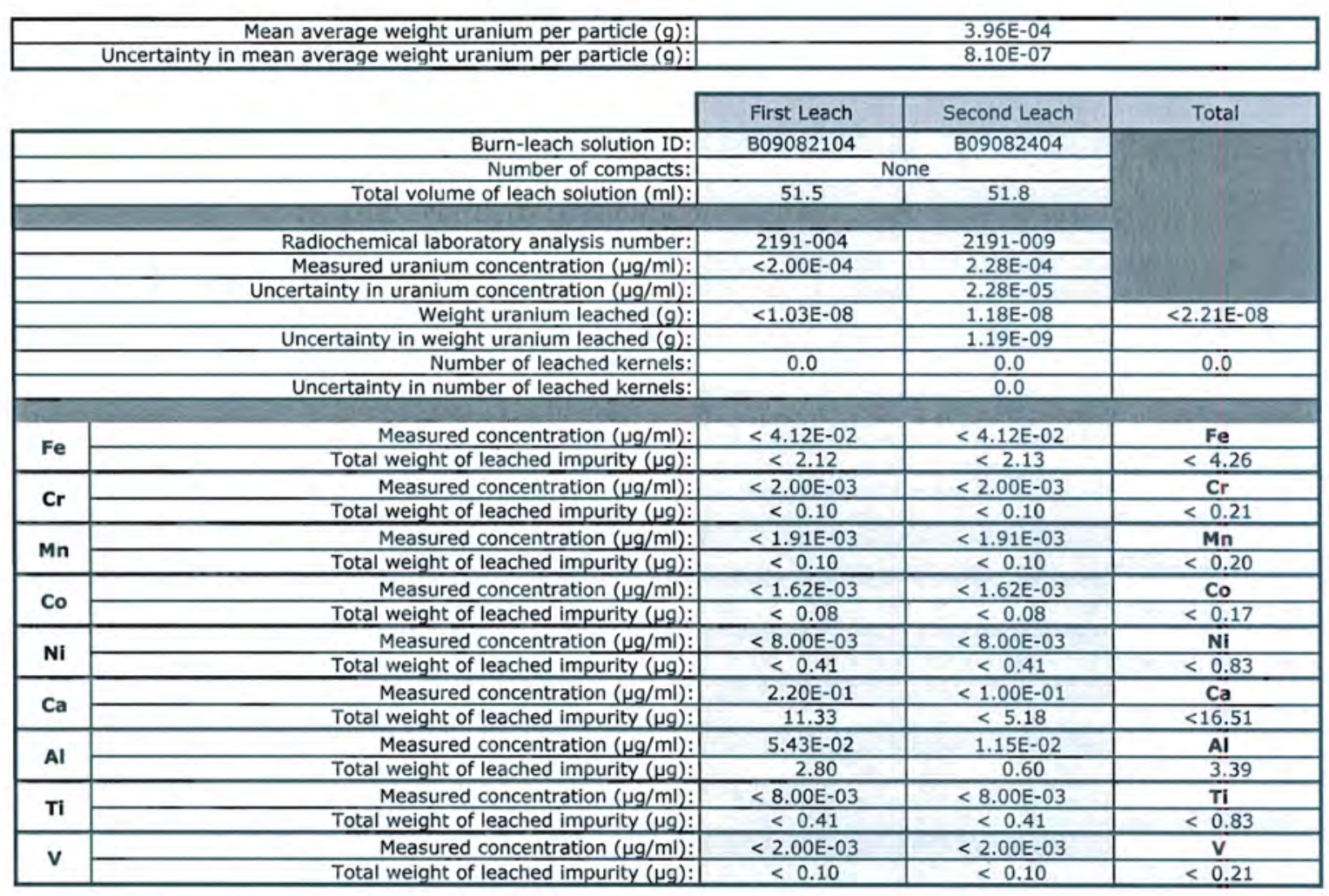

Comments

Data checked against RMAL 2191 official results of analyses by FCM on 10/14/2009

Fred c. montgomeny
$1-222010$

Date 
Data Report Form DRF-26A: Measurement of U Contamination or Impurities by Deconsolidation Leach

\begin{tabular}{|c|c|}
\hline $\begin{array}{l}\text { Procedure: } \\
\text { Operator: }\end{array}$ & AGR-CHAR-DAM-26 Rev. 1 \\
\hline $\begin{array}{r}\text { Operator: } \\
\text { Compact lot ID: }\end{array}$ & $\begin{array}{l}\text { Fred Montgomery } \\
\text { LEU09-OP2-Z }\end{array}$ \\
\hline Compact lot description: $\mathrm{A}$ & AGR-2 UCO Variant, from G73J-14-93073A \\
\hline Compact ID numbers: 1 & $118,041,138,007,071$ \\
\hline & 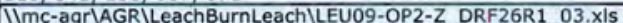 \\
\hline
\end{tabular}

\begin{tabular}{|c|c|c|c|c|}
\hline \multicolumn{2}{|r|}{$\begin{array}{c}\text { Mean average weight uranium per particle }(g): \\
\text { Uncertainty in mean average weight uranium per particle }(q):\end{array}$} & \multicolumn{3}{|c|}{$\begin{array}{c}3.964 \mathrm{E}-04 \\
8.1 \mathrm{E}-07 \\
\end{array}$} \\
\hline & & First Leach & Second Leach & Total \\
\hline & Deconsolidation-leach solution ID: & L09111001 & L09111601 & \\
\hline & Number of compacts: & & & \\
\hline & Total volume of leach solution (ml): & 117.0 & 117.0 & \\
\hline & Radiochemical laboratory analysis number: & $2392-001$ & 2392-006 & \\
\hline & Measured uranium concentration $(\mu \mathrm{g} / \mathrm{ml})$ : & $5.57 \mathrm{E}-02$ & $8.48 \mathrm{E}-03$ & \\
\hline & Uncertainty in uranium concentration $(\mu \mathrm{g} / \mathrm{ml})$ : & $5.57 \mathrm{E}-03$ & $8.48 \mathrm{E}-04$ & \\
\hline & Weight uranium leached $(g)$ : & $6.52 \mathrm{E}-06$ & $9.92 E-07$ & $7.51 \mathrm{E}-06$ \\
\hline & Uncertainty in weight uranium leached (g): & $6.53 \mathrm{E}-07$ & $9.94 \mathrm{E}-08$ & $6.60 \mathrm{E}-07$ \\
\hline & Effective number of exposed kernels: & 0.0 & 0.0 & 0.0 \\
\hline & Uncertainty in effective number of exposed kernels: & 0.0 & 0.0 & 0.0 \\
\hline \multirow{5}{*}{$\mathbf{F e}$} & Measured concentration of impurity in sample $(\mathrm{ug} / \mathrm{ml})$ : & $<4.12 \mathrm{E}-02,>20$ & & \\
\hline & Uncorrected weight of impurity in sample $(\mathrm{\mu g})$ : & $<4.82$ & $<4.82$ & $<9.64$ \\
\hline & Weight of impurity in blank $(\mu \mathrm{g})$ : & $<6.10$ & $<5.15$ & \\
\hline & Minimum corrected weight of impurity in sample $(\mu g)$ : & 0.00 & 0.00 & 0.00 \\
\hline & Maximum corrected welght of impurity in sample ( $(\mu g)$ : & 4.82 & 4.82 & 9.64 \\
\hline \multirow{5}{*}{$\mathbf{c r}$} & Measured concentration of impurity in sample $(\mu \mathrm{g} / \mathrm{ml})$ : & $1.08 \mathrm{E}-02$ & $6.08 \mathrm{E}-03$ & Cr \\
\hline & Uncorrected weight of impurity in sample $(\mu \mathrm{g})$ : & 1.26 & 0.71 & 1.97 \\
\hline & Weight of impurity in blank $(\mu g)$ : & 0.80 & $<0.25$ & \\
\hline & Minimum corrected weight of impurity in sample $(\mu \mathrm{g})$ : & 0.46 & 0.46 & 0.92 \\
\hline & Maximum corrected weight of impurity in sample $(\mu g)$ : & 0.46 & 0.71 & 1.17 \\
\hline \multirow{5}{*}{ Mn } & Measured concentration of impurity in sample $(\mathrm{\mu g} / \mathrm{ml})$ : & $<1.91 \mathrm{E}-03$ & $<1.91 \mathrm{E}-03$ & $\mathrm{Mn}$ \\
\hline & Uncorrected weight of impurity in sample $(\mu g)$ : & $<0.22$ & $<0.22$ & $<0.45$ \\
\hline & Weight of impurity in blank $(\mu \mathrm{g})$ : & $<0.28$ & $<0.24$ & \\
\hline & Minimum corrected weight of impurity in sample $(\mu \mathrm{g})$ : & 0.00 & 0.00 & 0.00 \\
\hline & Maximum corrected weight of impurity in sample $(\mu g)$ : & 0.22 & 0.22 & 0.45 \\
\hline \multirow{5}{*}{ co } & Measured concentration of impurity in sample $(\mu \mathrm{g} / \mathrm{ml})$ : & $<1.62 \mathrm{E}-03$ & $<1.62 \mathrm{E}-03$ & Co \\
\hline & Uncorrected weight of impurity in sample $(\mu g)$ : & $<0.19$ & $<0.19$ & $<0.38$ \\
\hline & $\begin{array}{l}\text { Weight of impurity in blank }(\mu \mathrm{g}) \text { : } \\
\end{array}$ & $<0.24$ & $<0.20$ & \\
\hline & Minimum corrected weight of Impurity in sample $(\mu g)$ : & 0.00 & 0.00 & 0.00 \\
\hline & Maximum corrected weight of impurity in sample $(\mu g)$ : & 0.19 & 0.19 & 0.38 \\
\hline \multirow{5}{*}{$\mathbf{N i}$} & Measured concentration of impurity in sample $(\mu \mathrm{g} / \mathrm{ml})$ : & $<8.00 \mathrm{E}-03$ & $<8.00 \mathrm{E}-03$ & $\mathrm{Ni}$ \\
\hline & Uncorrected weight of impurity in sample $(\mu g)$ : & $<0.94$ & $<0.94$ & $<1.87$ \\
\hline & Weight of impurity in blank $(\mu g)$ : & $<1.18$ & $<1.00$ & \\
\hline & Minimum corrected weight of impurity in sample $(\mu \mathrm{g})$ : & 0.00 & 0.00 & 0.00 \\
\hline & Maximum corrected weight of impurity in sample $(\mu \mathrm{g})$ : & 0.94 & 0.94 & 1.87 \\
\hline \multirow{5}{*}{$\mathbf{C a}$} & Measured concentration of impurity in sample $(\mu \mathrm{g} / \mathrm{ml})$ : & $5.02 \mathrm{E}-01$ & $7.86 \mathrm{E}-01$ & $\mathrm{Ca}$ \\
\hline & Uncorrected weight of impurity in sample $(\mu g)$ : & 58.73 & 91.96 & 150.70 \\
\hline & Weight of impurity in blank $(\mathrm{\mu g})$ : & 37.00 & $<12.50$ & \\
\hline & Minimum corrected weight of impurity in sample $(\mu \mathrm{g})$ : & 21.73 & 79.46 & 101.20 \\
\hline & Maximum corrected weight of impurity in sample $(\mu \mathrm{g})$ : & 21.73 & 91.96 & 113.70 \\
\hline \multirow{5}{*}{ Al } & Measured concentration of impurity in sample $(\mu \mathrm{g} / \mathrm{ml})$ : & $5.24 \mathrm{E}-01$ & $1.38 \mathrm{E}-01$ & Al \\
\hline & Uncorrected weight of impurity in sample $(\mu \mathrm{g})$ : & 61.31 & 16.15 & 77.45 \\
\hline & Weight of impurity in blank $(\mu \mathrm{g})$ : & 7.73 & 1.41 & \\
\hline & Minimum corrected weight of impurity in sample $(\mu \mathrm{g})$ : & 53.58 & 14.73 & 68.32 \\
\hline & Maximum corrected weight of impurity in sample $(\mu \mathrm{g})$ : & 53.58 & 14.73 & 68.32 \\
\hline \multirow{5}{*}{$\mathbf{T i}$} & Measured concentration of impurity in sample $(\mu \mathrm{g} / \mathrm{ml})$ : & $3.50 \mathrm{E}-02$ & $3.32 \mathrm{E}-02$ & $\mathrm{Ti}$ \\
\hline & Uncorrected weight of impurity in sample $(\mu \mathrm{g})$ : & 4.10 & 3.88 & 7.98 \\
\hline & Weight of impurity in blank $(\mu g)$ : & $<1.18$ & $<1.00$ & \\
\hline & Minimum corrected weight of impurity in sample ( $\mu \mathrm{g})$ : & 2.91 & 2.88 & 5.80 \\
\hline & Maximum corrected weight of impurity in sample $(\mu \mathrm{g})$ : & 4.10 & 3.88 & 7.98 \\
\hline \multirow{5}{*}{$\mathbf{v}$} & Measured concentration of impurity in sample $(\mu \mathrm{g} / \mathrm{ml})$ : & $2.34 \mathrm{E}-01$ & $5.16 \mathrm{E}-02$ & $\mathbf{v}$ \\
\hline & Uncorrected weight of impurity in sample $(\mu \mathrm{g})$ : & 27.38 & 6.04 & 33.42 \\
\hline & Weight of impurity in blank $(\mu g)$ : & $<0.30$ & $<0.25$ & \\
\hline & Minimum corrected weight of impurity in sample $(\mu \mathrm{g})$ : & 27.08 & 5.79 & 32.87 \\
\hline & Maximum corrected weight of impurity in sample $(\mu \mathrm{g})$ : & 27.38 & 6.04 & 33.42 \\
\hline
\end{tabular}

Comments

Data checked against RMAL 2392 official results of analyses by FCM on 12/03/2009

Faed c. Montgomery

$1-27-2010$

Date 
Data Report Form DRF-26A: Measurement of U Contamination or Impurlties by Deconsolidation Leach

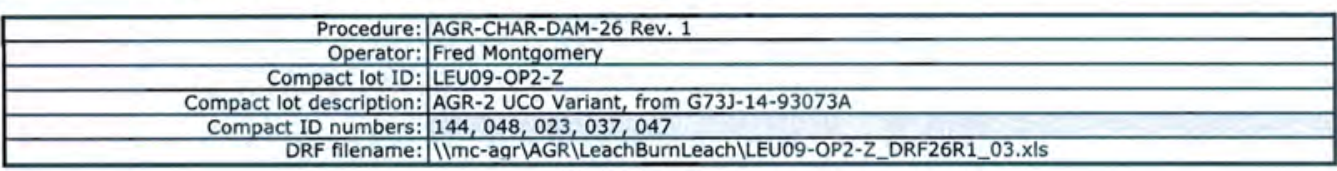

\begin{tabular}{|c|c|c|c|c|}
\hline \multicolumn{2}{|r|}{\begin{tabular}{|c|} 
Mean average weight uranium per particle $(\mathrm{g})$ : \\
Uncertainty in mean average weight uranium per particle $(\mathrm{q})$ :
\end{tabular}} & \multicolumn{3}{|c|}{$\begin{array}{l}3.96 E-04 \\
8.10 E-07\end{array}$} \\
\hline & & First Leach & Second Leach & Total \\
\hline & Deconsolidation-leach solution ID: & L09111002 & L09111602 & \\
\hline & Number of compacts: & & & \\
\hline & Total volume of leach solution $(\mathrm{ml}):$ & 124.0 & 129.0 & \\
\hline & Radiochemical laboratory analysis number: & $2392-002$ & $2392-007$ & \\
\hline & Measured uranium concentration $(\mathrm{\mu g} / \mathrm{ml})$ : & $5.59 \mathrm{E}-02$ & $7.19 \mathrm{E}-03$ & \\
\hline & Uncertainty in uranium concentration $(\mathrm{\mu g} / \mathrm{ml})$ : & $5.59 \mathrm{E}-03$ & $7.19 \mathrm{E}-04$ & \\
\hline & Weight uranium leached (g): & $6.93 E-06$ & $9.28 \mathrm{E}-07$ & $7.86 \mathrm{E}-06$ \\
\hline & Uncertainty in weight uranium leached $(\mathrm{g})$ : & $6.94 \mathrm{E}-07$ & $9.29 \mathrm{E}-08$ & $7.00 \mathrm{E}-07$ \\
\hline & Effective number of exposed kernels: & 0.0 & 0.0 & 0.0 \\
\hline & Uncertainty in effective number of exposed kernels: & 0.0 & 0.0 & 0.0 \\
\hline \multirow{5}{*}{$\mathbf{F e}$} & Measured concentration of impurity in sample $(\mathrm{\mu g} / \mathrm{ml})$ : & 4.68E-02 & $<4.12 \mathrm{E}-02$ & $\mathrm{Fe}$ \\
\hline & Uncorrected weight of impurity in sample $(\mu \mathrm{g})$ : & 5.80 & $<5.31$ & $<11.12$ \\
\hline & Weight of impurity in blank $(\mu g)$ : & $<6.10$ & $<5.15$ & \\
\hline & Minimum corrected weight of impurity in sample $(\mu \mathrm{g})$ : & 0.00 & 0.00 & 0.00 \\
\hline & Maximum corrected weight of impurity in sample $(\mu g)$ : & 5.80 & 5.31 & 11.12 \\
\hline \multirow{5}{*}{ Cr } & Measured concentration of impurity in sample $(\mathrm{\mu g} / \mathrm{ml})$ : & $1.53 \mathrm{E}-02$ & $5.24 \mathrm{E}-03$ & $\mathrm{Cr}$ \\
\hline & Uncorrected weight of impurity in sample $(\mu \mathrm{g})$ : & 1.90 & 0.68 & 2.57 \\
\hline & Weight of impurity in blank $(\mu g)$ : & 0.80 & $<0.25$ & \\
\hline & Minimum corrected weight of impurity in sample $(\mu g)$ : & 1.09 & 0.43 & 1.52 \\
\hline & Maximum corrected weight of impurity in sample $(\mu g)$ : & 1.09 & 0.68 & 1.77 \\
\hline \multirow{5}{*}{ Mn } & Measured concentration of impurity in sample $(\mu \mathrm{g} / \mathrm{ml})$ : & $<1.91 \mathrm{E}-03$ & $<1.91 \mathrm{E}-03$ & $\mathrm{Mn}$ \\
\hline & Uncorrected weight of impurity in sample $(\mu \mathrm{g})$ : & $<0.24$ & $<0.25$ & $<0.48$ \\
\hline & Weight of impurity in blank $(\mu \mathrm{g})$ : & $<0.28$ & $<0.24$ & \\
\hline & Minimum corrected weight of impurity in sample $(\mu g)$ : & 0.00 & 0.00 & 0.00 \\
\hline & Maximum corrected weight of impurity in sample $(\mu q)$ : & 0.24 & 0.25 & 0.48 \\
\hline \multirow{5}{*}{ co } & Measured concentration of impurity in sample $(\mathrm{\mu g} / \mathrm{ml})$ : & $<1.62 \mathrm{E}-03$ & $<1.62 \mathrm{E}-03$ & Co \\
\hline & Uncorrected weight of impurity in sample $(\mu \mathrm{g})$ : & $<0.20$ & $<0.21$ & $<0.41$ \\
\hline & $\begin{array}{c}\text { Weight of impurity in blank }(\mu \mathrm{g}) \text { : } \\
\end{array}$ & $<0.24$ & $<0.20$ & \\
\hline & Minimum corrected weight of impurity in sample $(\mu g)$ : & 0.00 & 0.00 & 0.00 \\
\hline & Maximum corrected weight of impurity in sample $(\mu g)$ : & 0.20 & 0.21 & 0.41 \\
\hline \multirow{5}{*}{$\mathrm{Ni}$} & Measured concentration of impurity in sample $(\mathrm{\mu g} / \mathrm{ml})$ : & $<8.00 \mathrm{E}-03$ & $<8.00 \mathrm{E}-03$ & $\mathrm{Ni}$ \\
\hline & Uncorrected weight of impurity in sample $(\mu g)$ : & $<0.99$ & $<1.03$ & $<2.02$ \\
\hline & Weight of impurity in blank $(\mu g)$ : & $<1.18$ & $<1.00$ & \\
\hline & Minimum corrected weight of impurity in sample $(\mu g)$ : & 0.00 & 0.00 & 0.00 \\
\hline & Maximum corrected weight of impurity in sample $(\mu g)$ : & 0.99 & 1.03 & 2.02 \\
\hline \multirow{5}{*}{$\mathbf{C a}$} & Measured concentration of impurity in sample $(\mathrm{\mu g} / \mathrm{ml})$ : & $3.67 \mathrm{E}-01$ & $4.10 \mathrm{E}-01$ & $\mathrm{Ca}$ \\
\hline & Uncorrected weight of impurity in sample $(\mu g)$ : & 45.51 & 52.89 & 98.40 \\
\hline & Weight of impurity in blank $(\mu \mathrm{g})$ : & 37.00 & $<12.50$ & \\
\hline & Minimum corrected weight of impurity in sample $(\mu g)$ : & 8.51 & 40.39 & 48.90 \\
\hline & Maximum corrected weight of impurity in sample $(\mu g)$ : & 8.51 & 52.89 & 61.40 \\
\hline \multirow{5}{*}{ Al } & Measured concentration of impurity in sample $(\mathrm{\mu g} / \mathrm{ml})$ : & $4.82 \mathrm{E}-01$ & $1.07 \mathrm{E}-01$ & Al \\
\hline & Uncorrected weight of impurity in sample $(\mu g)$ : & 59.77 & 13.80 & 73.57 \\
\hline & Weight of impurity in blank $(\mu \mathrm{g})$ : & 7.73 & 1.41 & \\
\hline & Minimum corrected weight of impurity in sample $(\mu g)$ : & 52.04 & 12.39 & 64.43 \\
\hline & Maximum corrected weight of impurity in sample $(\mu \mathrm{g})$ : & 52.04 & 12.39 & 64.43 \\
\hline \multirow{5}{*}{ Ti } & Measured concentration of impurity in sample $(\mathrm{\mu g} / \mathrm{ml})$ : & $3.18 \mathrm{E}-02$ & $2.47 \mathrm{E}-02$ & $\mathrm{Ti}$ \\
\hline & Uncorrected weight of impurity in sample $(\mu \mathrm{g})$ : & 3.94 & & 7.13 \\
\hline & Weight of impurity in blank $(\mu g)$ : & $<1.18$ & $<1.00$ & \\
\hline & Minimum corrected weight of impurity in sample $(\mu g)$ : & 2.76 & 2.19 & 4.95 \\
\hline & Maximum corrected weight of impurity in sample $(\mu g)$ : & 3.94 & 3.19 & 7.13 \\
\hline \multirow{5}{*}{$\mathbf{v}$} & Measured concentration of impurity in sample $(\mu \mathrm{g} / \mathrm{ml})$ : & $2.42 \mathrm{E}-01$ & $4.55 E-02$ & $\mathrm{v}$ \\
\hline & Uncorrected weight of impurity in sample $(\mu g)$ : & 30.01 & 5.87 & 35.88 \\
\hline & Weight of impurity in blank $(\mu \mathrm{g})$ : & $<0.30$ & $<0.25$ & \\
\hline & Minimum corrected weight of impurity in sample $(\mu \mathrm{g})$ : & 29.71 & 5.62 & 35.33 \\
\hline & Maximum corrected weight of impurity in sample $(\mu \mathrm{g})$ : & 30.01 & 5.87 & 35.88 \\
\hline \multicolumn{5}{|c|}{ Comments } \\
\hline & ked against RMAL 2392 official results of analyses by FCM & $2 / 03 / 2009$ & & \\
\hline
\end{tabular}

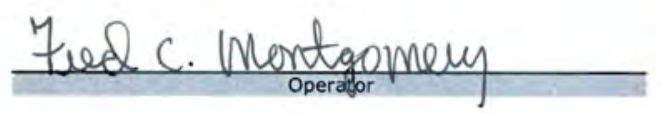

$1-27-2010$ 
Data Report Form DRF-26A: Measurement of U Contamination or Impuritles by Deconsolldation Leach

\begin{tabular}{|c|c|}
\hline $\begin{array}{c}\text { Procedure: } \\
\text { Operator: }\end{array}$ & $\begin{array}{l}\text { AGR-CHAR-DAM-26 Rev. } 1 \\
\text { Fred Montgomery }\end{array}$ \\
\hline Compact lot ID: & LEUOS-OP2-Z \\
\hline Compact lot description: & AGR-2 UCO Variant, from G73J-14-93073A \\
\hline Compact ID numbers: & $150,065,116,140,160$ \\
\hline
\end{tabular}

\begin{tabular}{|c|c|c|c|c|}
\hline & $\begin{array}{l}\text { Mean average weight uranium per particle (g): } \\
\text { Uncertainty in mean average weight uranium per particle (q): }\end{array}$ & & $\begin{array}{l}3.96 \mathrm{E}-04 \\
8.10 \mathrm{E}-07\end{array}$ & \\
\hline & & First Leach & Second Leach & Total \\
\hline & Deconsolidation-leach solution ID: & L09111004 & L09111604 & \\
\hline & Number of compacts: & & & \\
\hline & Total volume of leach solution $(\mathrm{ml})$ : & 130.0 & 131.0 & \\
\hline & Radiochemical laboratory analysis number: | & $2392-004$ & $2392-009$ & \\
\hline & Measured uranium concentration $(\mu \mathrm{g} / \mathrm{ml})$ : & $2.46 E+00$ & $1.06 \mathrm{E}-01$ & \\
\hline & Uncertainty in uranium concentration $(\mu \mathrm{g} / \mathrm{ml}):$ & $2.46 \mathrm{E}-01$ & $1.06 \mathrm{E}-02$ & \\
\hline & Weight uranium leached $(\mathrm{g})$ : & $3.20 \mathrm{E}-04$ & $1.39 \mathrm{E}-05$ & $3.34 \mathrm{E}-04$ \\
\hline & Uncertainty in weight uranium leached (g): & $3.20 \mathrm{E}-05$ & $1.39 \mathrm{E}-06$ & $3.21 \mathrm{E}-05$ \\
\hline & Effective number of exposed kernels: & 0.8 & 0.0 & 0.8 \\
\hline & Uncertainty in effective number of exposed kernels: & 0.1 & 0.0 & 0.1 \\
\hline & Measured concentration of impurity in sample $(\mu \mathrm{g} / \mathrm{ml})$ : & $<4.12 \mathrm{E}-02$ & $<4.12 \mathrm{E}-02$ & $\mathrm{Fe}$ \\
\hline & Uncorrected weight of impurity in sample $(\mu \mathrm{g})$ : & $<5.36$ & $<5.40$ & $<10.75$ \\
\hline $\mathbf{F e}$ & Weight of impurity in blank $(\mu \mathrm{g})$ : & $<6.10$ & $<5.15$ & \\
\hline & Minimum corrected weight of impurity in sample $(\mu g)$ : & 0.00 & 0.00 & 0.00 \\
\hline & Maximum corrected weight of impurity in sample $(\mu \mathrm{g})$ : & 5.36 & 5.40 & 10.75 \\
\hline & Measured concentration of impurity in sample $(\mathrm{\mu g} / \mathrm{ml})$ : & $1.15 \mathrm{E}-02$ & $5.92 \mathrm{E}-03$ & $\mathrm{Cr}$ \\
\hline & Uncorrected weight of impurity in sample $(\mu \mathrm{g})$ : & 1.50 & 0.78 & 2.27 \\
\hline cr & Weight of impurity in blank $(\mu g)$ : & 0.80 & $<0.25$ & \\
\hline & Minimum corrected weight of impurity in sample $(\mu \mathrm{g})$ : & 0.69 & 0.53 & 1.22 \\
\hline & Maximum corrected weight of impurity in sample $(\mu \mathrm{g})$ : & 0.69 & 0.78 & 1.47 \\
\hline & Measured concentration of impurity in sample $(\mathrm{\mu g} / \mathrm{ml})$ : & $<1.91 \mathrm{E}-03$ & $<1.91 \mathrm{E}-03$ & $\mathrm{Mn}$ \\
\hline & Uncorrected weight of impurity in sample $(\mu \mathrm{g})$ : & $<0.25$ & $<0.25$ & $<0.50$ \\
\hline Mn & Weight of impurity in blank $(\mu \mathrm{g})$ : & $<0.28$ & $<0.24$ & \\
\hline & Minimum corrected weight of impurity in sample $(\mu \mathrm{g})$ : & 0.00 & 0.00 & 0.00 \\
\hline & Maximum corrected weight of impurity in sample $(\mu \mathrm{g})$ : & 0.25 & 0.25 & 0.50 \\
\hline & Measured concentration of impurity in sample $(\mu \mathrm{g} / \mathrm{ml})$ : & $<1.62 \mathrm{E}-03$ & $<1.62 \mathrm{E}-03$ & Co \\
\hline & Uncorrected weight of impurity in sample $(\mu g)$ : & $<0.21$ & $<0.21$ & $<0.42$ \\
\hline Co & Weight of impurity in blank $(\mu \mathrm{g})$ : & $<0.24$ & $<0.20$ & \\
\hline & Minimum corrected weight of impurity in sample $(\mu \mathrm{g})$ : & 0.00 & 0.00 & 0.00 \\
\hline & Maximum corrected weight of impurity in sample $(\mu \mathrm{g})$ : & 0.21 & 0.21 & 0.42 \\
\hline & Measured concentration of impurity in sample $(\mu \mathrm{g} / \mathrm{ml})$ : & $<8.00 \mathrm{E}-03$ & $<8.00 \mathrm{E}-03$ & $\mathrm{Ni}$ \\
\hline & Uncorrected weight of impurity in sample $(\mu \mathrm{g})$ : & $<1.04$ & $<1.05$ & $<2.09$ \\
\hline $\mathbf{N i}$ & Weight of impurity in blank $(\mu \mathrm{g})$ : & $<1.18$ & $<1.00$ & \\
\hline & Minimum corrected weight of impurity in sample $(\mu g)$ : & 0.00 & 0.00 & 0.00 \\
\hline & Maximum corrected weight of impurity in sample $(\mu \mathrm{g})$ : & 1.04 & 1.05 & 2.09 \\
\hline & Measured concentration of impurity in sample $(\mathrm{\mu g} / \mathrm{ml})$ : & $6.17 E-01$ & $1.66 \mathrm{E}-01$ & $\mathrm{Ca}$ \\
\hline & Uncorrected weight of impurity in sample $(\mu \mathrm{g})$ : & 80.21 & 21.75 & 101.96 \\
\hline $\mathbf{C a}$ & Weight of impurity in blank $(\mu \mathrm{g})$ : & 37.00 & $<12.50$ & \\
\hline & Minimum corrected weight of impurity in sample $(\mu \mathrm{g})$ : & 43.21 & 9.25 & 52.46 \\
\hline & Maximum corrected weight of impurity in sample $(\mu g)$ : & 43.21 & 21.75 & 64.96 \\
\hline & Measured concentration of impurity in sample $(\mathrm{\mu g} / \mathrm{ml})$ : & $4.92 \mathrm{E}-01$ & $1.19 \mathrm{E}-01$ & Al \\
\hline & Uncorrected weight of impurity in sample $(\mu \mathrm{g})$ : & 63.96 & 15.59 & 79.55 \\
\hline Al & Weight of impurity in blank $(\mu \mathrm{g})$ : & 7.73 & 1.41 & \\
\hline & Minimum corrected weight of impurity in sample $(\mu g)$ : & 56.23 & 14.18 & 70.41 \\
\hline & Maximum corrected weight of impurity in sample $(\mu q)$ : & 56.23 & 14.18 & 70.41 \\
\hline & Measured concentration of impurity in sample $(\mathrm{\mu g} / \mathrm{ml})$ : & $4.16 \mathrm{E}-02$ & $2.62 \mathrm{E}-02$ & $\mathrm{Ti}$ \\
\hline & Uncorrected weight of impurity in sample $(\mu \mathrm{g})$ : & 5.41 & 3.43 & 8.84 \\
\hline $\mathbf{T i}$ & Weight of impurity in blank $(\mu g)$ : & $<1.18$ & $<1.00$ & \\
\hline & Minimum corrected weight of impurity in sample $(\mu \mathrm{g})$ : & 4.22 & 2.43 & 6.66 \\
\hline & Maximum corrected weight of impurity in sample $(\mu g)$ : & 5.41 & 3.43 & 8.84 \\
\hline & Measured concentration of impurity in sample $(\mu \mathrm{g} / \mathrm{ml})$ : & $2.28 \mathrm{E}-01$ & $4.34 \mathrm{E}-02$ & $\mathbf{v}$ \\
\hline & Uncorrected weight of impurity in sample $(\mu g)$ : & 29.64 & 5.69 & 35.33 \\
\hline v & Weight of Impurity in blank $(\mu \mathrm{g})$ : & $<0.30$ & $<0.25$ & \\
\hline & Minimum corrected weight of impurity in sample $(\mu g)$ : & 29.34 & 5.44 & 34.78 \\
\hline & Maximum corrected weight of impurity in sample $(\mu \mathrm{g})$ : & 29.64 & 5.69 & 35.33 \\
\hline & & & & \\
\hline & ked against RMAL 2392 official results of analyses by FCM 0 & $03 / 2009$ & & \\
\hline
\end{tabular}

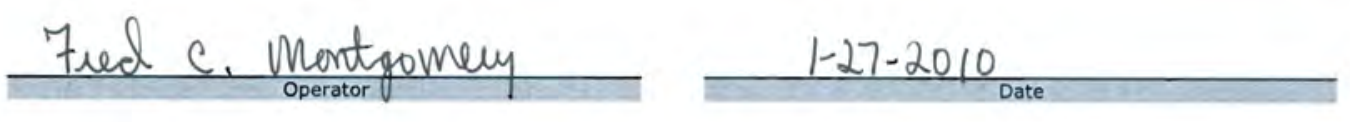


Data Report Form DRF-26A: Measurement of U Contamination or Impurities by Deconsolldation Leach

\begin{tabular}{|c|c|}
\hline Procedure: & AGR-CHAR-DAM-26 Rev. 1 \\
\hline Operator: & Fred Montgomery \\
\hline Compact lot ID: & LEU09-OP2-Z \\
\hline Compact lot description: & AGR-2 UCO Variant, from G73J-14-93073A \\
\hline Compact ID numbers: & $034,084,074,159,060$ \\
\hline DRF filename: & IImc-agr\AGR\LeachBurnLeach|LEU09-OP2-Z_DRF26R1_03.xIs \\
\hline
\end{tabular}

\begin{tabular}{|c|c|c|c|c|}
\hline \multirow{2}{*}{\multicolumn{2}{|c|}{$\begin{array}{r}\text { Mean average weight uranium per particle }(\mathrm{g}): \\
\text { Uncertainty in mean averaqe weight uranium per particle }(\mathrm{q}):\end{array}$}} & \multicolumn{3}{|c|}{$3.96 \mathrm{E}-04$} \\
\hline & & \multicolumn{3}{|c|}{$8.10 \mathrm{E}-07$} \\
\hline & & First Leach & Second Leach & Total \\
\hline & Deconsolidation-leach solution ID: & L09111005 & L09111605 & \\
\hline & Number of compacts: & & & \\
\hline & Total volume of leach solution $(\mathrm{ml})$ : & 128.0 & 122.0 & \\
\hline & Radiochemical laboratory analysis number: & $2392-005$ & $2392-010$ & \\
\hline & Measured uranium concentration $(\mathrm{\mu g} / \mathrm{ml})$ : & $5.39 \mathrm{E}-02$ & $8.57 E-03$ & \\
\hline & Uncertainty in uranium concentration $(\mu \mathrm{g} / \mathrm{ml})$ : & $5.39 E-03$ & 8.57E-04 & \\
\hline & Weight uranium leached $(\mathrm{g})$ : & $6.90 E-06$ & $1.05 E-06$ & $7.94 \mathrm{E}-06$ \\
\hline & Uncertainty in weight uranium leached $(g)$ : & $6.91 \mathrm{E}-07$ & $1.05 E-07$ & $6.99 \mathrm{E}-07$ \\
\hline & Effective number of exposed kernels: & 0.0 & 0.0 & 0.0 \\
\hline & Uncertainty in effective number of exposed kernels: & 0.0 & 0.0 & 0.0 \\
\hline \multirow{5}{*}{$\mathbf{F e}$} & Measured concentration of impurity in sample $(\mathrm{\mu g} / \mathrm{ml})$ : & $<4.12 \mathrm{E}-02$ & $<4.12 \mathrm{E}-02$ & $\mathrm{Fe}$ \\
\hline & Uncorrected weight of impurity in sample $(\mu g)$ : & $<5.27$ & $<5.03$ & $<10.30$ \\
\hline & Weight of impurity in blank $(\mu \mathrm{g})$ : & $<6.10$ & $<5.15$ & \\
\hline & Minimum corrected weight of impurity in sample $(\mu \mathrm{g})$ : & 0.00 & 0.00 & 0.00 \\
\hline & Maximum corrected weight of impurity in sample $(\mu \mathrm{g})$ : & 5.27 & 5.03 & 10.30 \\
\hline \multirow{5}{*}{$\mathbf{C r}$} & Measured concentration of impurity in sample $(\mu \mathrm{g} / \mathrm{ml})$ : & $1.26 \mathrm{E}-02$ & $5.21 E-03$ & $\mathrm{Cr}$ \\
\hline & Uncorrected weight of impurity in sample $(\mu \mathrm{g})$ : & 1.61 & 0.64 & 2.25 \\
\hline & Weight of impurity in blank $(\mu \mathrm{g})$ : & 0.80 & $<0.25$ & \\
\hline & Minimum corrected weight of impurity in sample $(\mu \mathrm{g})$ : & 0.81 & 0.39 & 1.19 \\
\hline & Maximum corrected weight of impurity in sample $(\mu \mathrm{g})$ : & 0.81 & 0.64 & 1.44 \\
\hline \multirow{5}{*}{ Mn } & Measured concentration of impurity in sample $(\mu \mathrm{g} / \mathrm{ml})$ : & $<1.91 \mathrm{E}-03$ & $<1.91 \mathrm{E}-03$ & $\mathrm{Mn}$ \\
\hline & Uncorrected weight of impurity in sample $(\mu \mathrm{g})$ : & $<0.24$ & $<0.23$ & $<0.48$ \\
\hline & Weight of impurity in blank $(\mu \mathrm{g})$ : & $<0.28$ & $<0.24$ & \\
\hline & Minimum corrected weight of impurity in sample $(\mu \mathrm{g})$ : & 0.00 & 0.00 & 0.00 \\
\hline & Maximum corrected weight of impurity in sample $(\mu \mathrm{g})$ : & 0.24 & 0.23 & 0.48 \\
\hline \multirow{5}{*}{ Co } & Measured concentration of impurity in sample $(\mu \mathrm{g} / \mathrm{ml})$ : & $<1.62 \mathrm{E}-03$ & $<1.62 \mathrm{E}-03$ & Co \\
\hline & Uncorrected weight of Impurity in sample $(\mu \mathrm{g})$ : & $<0.21$ & $<0.20$ & $<0.41$ \\
\hline & Weight of impurity in blank $(\mu \mathrm{g})$ : & $<0.24$ & $<0.20$ & 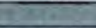 \\
\hline & Minimum corrected weight of impurity in sample $(\mu \mathrm{g})$ : & 0.00 & 0.00 & 0.00 \\
\hline & Maximum corrected weight of impurity in sample $(\mu \mathrm{g})$ : & 0.21 & 0.20 & 0.41 \\
\hline \multirow{5}{*}{ Ni } & Measured concentration of impurity in sample $(\mu \mathrm{g} / \mathrm{ml})$ : & $8.02 E-03$ & $<8.00 \mathrm{E}-03$ & $\mathrm{Ni}$ \\
\hline & Uncorrected weight of impurity in sample $(\mu \mathrm{g})$ : & 1.03 & $<0.98$ & $<2.00$ \\
\hline & Weight of impurity in blank $(\mu \mathrm{g})$ : & $<1.18$ & $<1.00$ & \\
\hline & Minimum corrected weight of impurity in sample $(\mu \mathrm{g})$ : & 0.00 & 0.00 & 0.00 \\
\hline & Maximum corrected weight of impurity in sample $(\mu \mathrm{g})$ : & 1.03 & 0.98 & 2.00 \\
\hline \multirow{5}{*}{$\mathbf{C a}$} & Measured concentration of impurity in sample $(\mu \mathrm{g} / \mathrm{ml})$ : & $1.05 E+00$ & $7.33 \mathrm{E}-01$ & $\mathrm{Ca}$ \\
\hline & Uncorrected weight of impurity in sample $(\mu \mathrm{g})$ : & 134.40 & 89.43 & 223.83 \\
\hline & Weight of impurity in blank $(\mu \mathrm{g})$ : & 37.00 & $<12.50$ & \\
\hline & Minimum corrected weight of impurity in sample $(\mu \mathrm{g})$ : & 97.40 & 76.93 & 174.33 \\
\hline & Maximum corrected weight of impurity in sample $(\mu \mathrm{g})$ : & 97.40 & 89.43 & 186.83 \\
\hline \multirow{5}{*}{ Al } & Measured concentration of impurity in sample $(\mu \mathrm{g} / \mathrm{ml})$ : & $5.24 \mathrm{E}-01$ & $1.36 \mathrm{E}-01$ & Al \\
\hline & Uncorrected weight of impurity in sample $(\mu \mathrm{g})$ : & 67.07 & 16.59 & 83.66 \\
\hline & Weight of impurity in blank $(\mu \mathrm{g})$ : & 7.73 & 1.41 & \\
\hline & Minimum corrected weight of impurity in sample $(\mu g)$ : & 59.35 & 15.18 & 74.53 \\
\hline & Maximum corrected weight of impurity in sample $(\mu \mathrm{g})$ : & 59.35 & 15.18 & 74.53 \\
\hline \multirow{5}{*}{$\mathbf{T i}$} & Measured concentration of impurity in sample $(\mu \mathrm{g} / \mathrm{ml})$ : & $5.38 \mathrm{E}-02$ & $3.05 E-02$ & $\mathrm{Ti}$ \\
\hline & Uncorrected weight of impurity in sample $(\mu \mathrm{g})$ : & 6.89 & 3.72 & 10.61 \\
\hline & Weight of impurity in blank $(\mu \mathrm{g})$ : & $<1.18$ & $<1.00$ & \\
\hline & Minimum corrected weight of impurity in sample $(\mu \mathrm{g})$ : & 5.70 & 2.72 & 8.42 \\
\hline & Maximum corrected weight of impurity in sample $(\mu \mathrm{g})$ : & 6.89 & 3.72 & 10.61 \\
\hline \multirow{5}{*}{$\mathbf{v}$} & Measured concentration of impurity in sample $(\mu \mathrm{g} / \mathrm{ml})$ : & $2.54 \mathrm{E}-01$ & $4.76 \mathrm{E}-02$ & $\mathbf{v}$ \\
\hline & Uncorrected weight of impurity in sample $(\mu \mathrm{g})$ : & 32.51 & 5.81 & 38.32 \\
\hline & Weight of impurity in blank $(\mu \mathrm{g})$ : & $<0.30$ & $<0.25$ & \\
\hline & Minimum corrected weight of impurity in sample $(\mu \mathrm{g})$ : & 32.22 & 5.56 & 37.77 \\
\hline & Maximum corrected weight of impurity In sample $(\mu \mathrm{g})$ : & 32.51 & 5.81 & 38.32 \\
\hline
\end{tabular}

Comments

\begin{tabular}{|l|}
\hline Data checked against RMAL 2392 official results of analyses by FCM on 12/03/2009 \\
\hline
\end{tabular} 
Data Report Form DRF-26A: Measurement of U Contamination or Impurities by Deconsolidation Leach

\begin{tabular}{|r|l|}
\hline Procedure: & AGR-CHAR-DAM-26 Rev. 1 \\
\hline Operator: & Fred Montgomery \\
\hline Compact lot ID: & LEU09-OP2-Z \\
\hline Compact lot description: & AGR-2 UCO Variant, from G73J-14-93073A \\
\hline Compact ID numbers: & Deconsolidation Leach Blank \\
\hline DRF filename: & IImc-agr\AGR\LeachBurnLeach|LEU09-OP2-Z_DRF26R1_03.xIs \\
\hline
\end{tabular}

\begin{tabular}{|c|c|c|c|c|}
\hline & Mean average weight uranium per particle $(\mathrm{g})$ : & \multicolumn{3}{|c|}{$3.96 \mathrm{E}-04$} \\
\hline & Uncertainty in mean average weight uranium per particle (g): & \multicolumn{3}{|c|}{$8.10 \mathrm{E}-07$} \\
\hline & & First Leach & Second Leach & Total \\
\hline & Deconsolidation-leach solution ID: & L09111003 & L09111603 & \\
\hline & Number of compacts: & \multicolumn{2}{|c|}{ None } & \\
\hline & Total volume of leach solution $(\mathrm{ml})$ : & 148.0 & 125.0 & \\
\hline & Radiochemical laboratory analysis number: & $2392-003$ & $2392-008$ & \\
\hline & Measured uranium concentration $(\mu \mathrm{g} / \mathrm{ml})$ : & $3.96 \mathrm{E}-04$ & $<2.00 \mathrm{E}-04$ & \\
\hline & Uncertainty in uranium concentration $(\mu \mathrm{g} / \mathrm{ml})$ : & $3.96 \mathrm{E}-05$ & & \\
\hline & Weight uranium leached $(g)$ : & $5.86 \mathrm{E}-08$ & $<2.50 \mathrm{E}-08$ & $<8.36 \mathrm{E}-08$ \\
\hline & Uncertainty in weight uranium leached $(\mathrm{g})$ : & 5.87E-09 & & \\
\hline & Effective number of exposed kernels: & 0.0 & 0.0 & 0.0 \\
\hline & Uncertainty in effective number of exposed kernels: & 0.0 & & \\
\hline \multirow[b]{2}{*}{$\mathrm{Fe}$} & Measured concentration $(\mu \mathrm{g} / \mathrm{ml})$ : & $<4.12 \mathrm{E}-02$ & $<4.12 \mathrm{E}-02$ & $\mathbf{F e}$ \\
\hline & Total weight of leached impurity $(\mu \mathrm{g})$ : & $<6.10$ & $<5.15$ & $<11.25$ \\
\hline \multirow{2}{*}{$\mathbf{C r}$} & Measured concentration $(\mu \mathrm{g} / \mathrm{ml})$ : & $5.43 E-03$ & $<2.00 \mathrm{E}-03$ & $\mathrm{Cr}$ \\
\hline & Total weight of leached impurity $(\mu \mathrm{g})$ : & 0.80 & $<0.25$ & $<1.05$ \\
\hline \multirow{2}{*}{ Mn } & Measured concentration $(\mu \mathrm{g} / \mathrm{ml})$ : & $<1.91 \mathrm{E}-03$ & $<1.91 \mathrm{E}-03$ & Mn \\
\hline & Total weight of leached impurity $(\mu \mathrm{g})$ : & $<0.28$ & $<0.24$ & $<0.52$ \\
\hline \multirow{2}{*}{ Co } & Measured concentration $(\mu \mathrm{g} / \mathrm{ml})$ : & $<1.62 \mathrm{E}-03$ & $<1.62 \mathrm{E}-03$ & Co \\
\hline & Total weight of leached impurity $(\mu \mathrm{g})$ : & $<0.24$ & $<0.20$ & $\leq 0.44$ \\
\hline \multirow{2}{*}{$\mathbf{N i}$} & Measured concentration $(\mu \mathrm{g} / \mathrm{ml})$ : & $<8.00 \mathrm{E}-03$ & $<8.00 \mathrm{E}-03$ & $\mathrm{Ni}$ \\
\hline & Total weight of leached impurity $(\mu \mathrm{g})$ : & $<1.18$ & $<1.00$ & $<2.18$ \\
\hline \multirow{2}{*}{$\mathrm{Ca}$} & Measured concentration $(\mu \mathrm{g} / \mathrm{ml})$ : & $2.50 \mathrm{E}-01$ & $<1.00 \mathrm{E}-01$ & $\mathrm{Ca}$ \\
\hline & Total weight of leached impurity $(\mu \mathrm{g})$ : & 37.00 & $<12.50$ & $<49.50$ \\
\hline \multirow{2}{*}{ Al } & Measured concentration $(\mu \mathrm{g} / \mathrm{ml})$ : & $5.22 \mathrm{E}-02$ & $1.13 E-02$ & Al \\
\hline & Total weight of leached impurity $(\mu \mathrm{g})$ : & 7.73 & 1.41 & 9.14 \\
\hline \multirow{2}{*}{$\mathbf{T i}$} & Measured concentration $(\mu \mathrm{g} / \mathrm{ml})$ : & $<8.00 \mathrm{E}-03$ & $<8.00 \mathrm{E}-03$ & $\mathrm{Ti}$ \\
\hline & Total weight of leached impurity $(\mu \mathrm{g})$ : & $<1.18$ & $<1.00$ & $<2.18$ \\
\hline \multirow{2}{*}{$\mathbf{v}$} & Measured concentration $(\mu \mathrm{g} / \mathrm{ml})$ : & $<2.00 \mathrm{E}-03$ & $<2.00 \mathrm{E}-03$ & $\mathbf{v}$ \\
\hline & Total weight of leached impurity $(\mu \mathrm{g})$ : & $<0.30$ & $<0.25$ & $<0.55$ \\
\hline
\end{tabular}

Comments

Data checked against RMAL 2392 official results of analyses by FCM on 12/03/2009

Freed c. Montgomely
$1-27-2010$

Date 


\begin{tabular}{|r|l|}
\hline Procedure: & AGR-CHAR-DAM-26 Rev. 1 \\
\hline Operator: & Fred Montgomery \\
\hline Compact lot ID: & LEU09-OP2-Z \\
\hline Compact lot description: & AGR-2 UCO Variant, from G73J-14-93073A \\
\hline Compact ID numbers: & $050,044,020,164,098$ \\
\hline DRF filename: & IImc-agrIAGRILeachBurnLeachILEU09-OP2-Z-DRF26R1_04.xIs \\
\hline
\end{tabular}

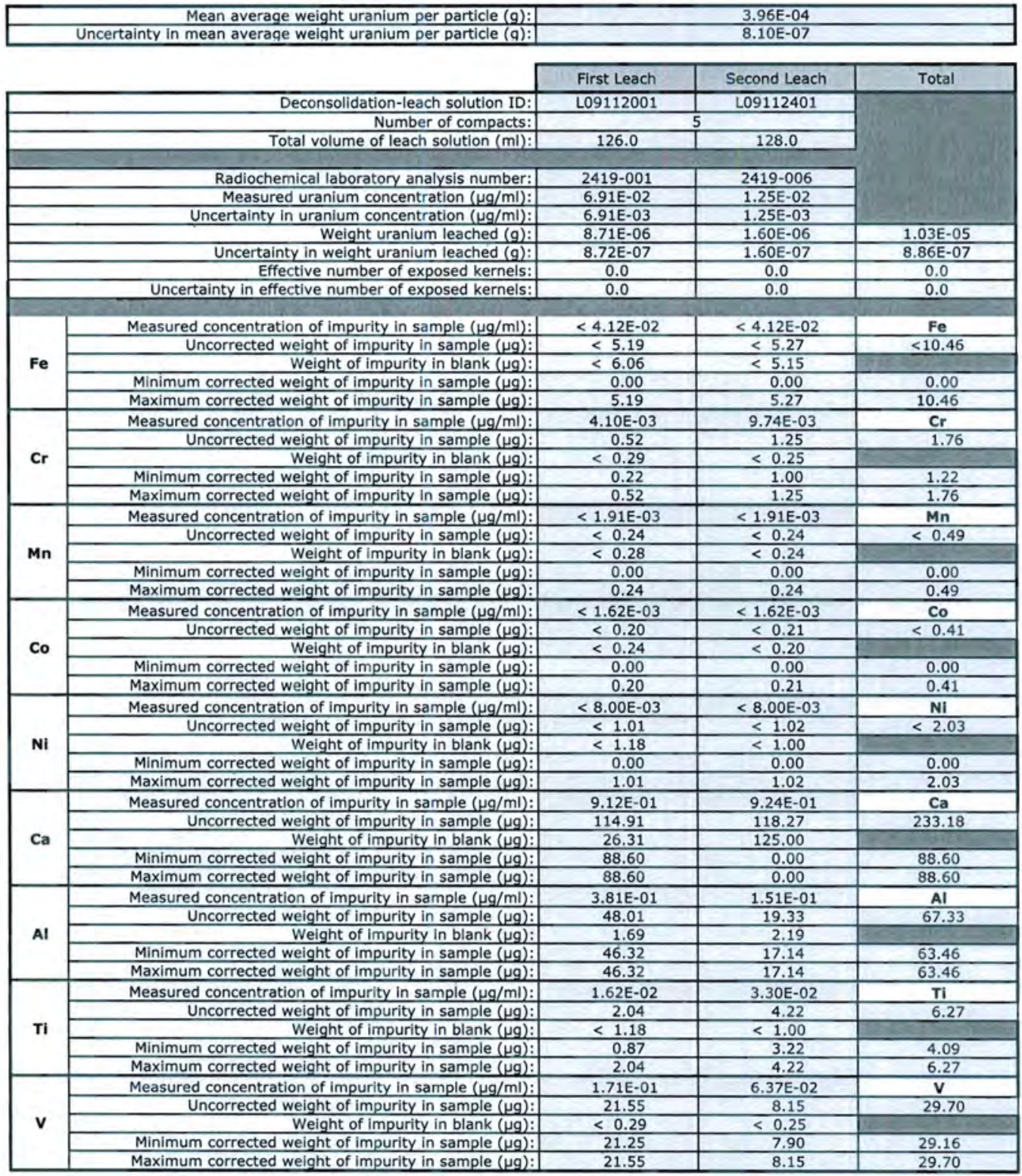

Comments

Data checked against RMAL 2419 official results of analyses by FCM on 12/09/2009 
Data Report Form DRF-26A: Measurement of U Contamination or Impurities by Deconsolidation Leach

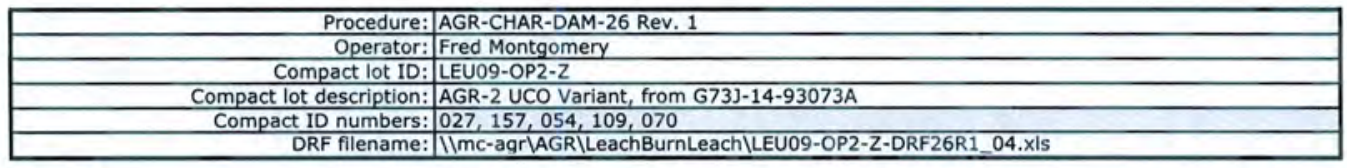

\begin{tabular}{|c|c|c|c|c|}
\hline \multicolumn{2}{|r|}{$\begin{array}{c}\text { Mean average weight uranium per particle }(g) \text { : } \\
\text { Uncertainty in mean average weiqht uranium per particle }(q) \text { : }\end{array}$} & \multicolumn{3}{|c|}{$\begin{array}{l}3.96 E-04 \\
8.10 E-07\end{array}$} \\
\hline & & First Leach & Second Leach & Total \\
\hline & Deconsolidation-leach solution ID: & L09112002 & L09112402 & \\
\hline & Number of compacts: & & & \\
\hline & Total volume of leach solution (ml): & 121.0 & 123.0 & \\
\hline & Radiochemical laboratory analysis number: & $2419-002$ & $2419-007$ & \\
\hline & Measured uranium concentration $(\mathrm{\mu g} / \mathrm{ml})$ : & $2.83 E+00$ & $1.59 \mathrm{E}-01$ & \\
\hline & Uncertainty in uranium concentration $(\mu \mathrm{g} / \mathrm{ml})$ : & $2.83 \mathrm{E}-01$ & $1.59 \mathrm{E}-02$ & \\
\hline & $\begin{array}{c}\text { Weight uranium leached }(g) \text { : } \\
\end{array}$ & $3.42 \mathrm{E}-04$ & $1.96 \mathrm{E}-05$ & $3.62 \mathrm{E}-04$ \\
\hline & Uncertainty in weight uranium leached $(\mathrm{g})$ : & $3.43 \mathrm{E}-05$ & $1.96 \mathrm{E}-06$ & $3.43 \mathrm{E}-05$ \\
\hline & Effective number of exposed kernels: & 0.9 & 0.0 & 0.9 \\
\hline & Uncertainty in effective number of exposed kernels: & 0.1 & 0.0 & 0.1 \\
\hline \multirow{5}{*}{$\mathbf{F e}$} & Measured concentration of impurity in sample $(\mu \mathrm{g} / \mathrm{ml})$ : & $<4.12 \mathrm{E}-02$ & $<4.12 \mathrm{E}-02$ & \\
\hline & Uncorrected weight of impurity in sample $(\mu \mathrm{g})$ : & $<4.99$ & $<5.07$ & $<10.05$ \\
\hline & Weight of impurity in blank $(\mu \mathrm{g})$ : & $<6.06$ & $<5.15$ & \\
\hline & Minimum corrected weight of impurity in sample $(\mu \mathrm{g})$ : & 0.00 & 0.00 & 0.00 \\
\hline & Maximum corrected weight of impurity in sample ( $(\mathrm{gg})$ : & 4.99 & 5.07 & 10.05 \\
\hline \multirow{5}{*}{$\mathbf{C r}$} & Measured concentration of impurity in sample $(\mu \mathrm{g} / \mathrm{ml})$ : & $9.84 \mathrm{E}-03$ & $7.38 \mathrm{E}-03$ & $\mathrm{Cr}$ \\
\hline & Uncorrected weight of impurity in sample $(\mu \mathrm{g})$ : & 1.19 & 0.91 & 2.10 \\
\hline & Weight of impurity in blank $(\mu \mathrm{g})$ : & $<0.29$ & $<0.25$ & \\
\hline & Minimum corrected weight of impurity in sample $(\mathrm{\mu g})$ : & 0.90 & 0.66 & 1.55 \\
\hline & Maximum corrected weight of impurity in sample $(\mu \mathrm{g})$ : & 1.19 & 0.91 & 2.10 \\
\hline \multirow{5}{*}{ Mn } & Measured concentration of impurity in sample $(\mu \mathrm{g} / \mathrm{ml})$ : & $<1.91 \mathrm{E}-03$ & $<1.91 \mathrm{E}-03$ & $\mathrm{Mn}$ \\
\hline & Uncorrected weight of impurity in sample $(\mu \mathrm{g})$ : & $<0.23$ & $<0.23$ & $<0.47$ \\
\hline & Weight of impurity in blank $(\mathrm{\mu g})$ : & $<0.28$ & $<0.24$ & \\
\hline & Minimum corrected weight of impurity in sample $(\mu \mathrm{g})$ : & 0.00 & 0.00 & 0.00 \\
\hline & Maximum corrected weight of impurity in sample $(\mathrm{\mu g})$ : & 0.23 & 0.23 & 0.47 \\
\hline \multirow{5}{*}{ co } & Measured concentration of impurity in sample $(\mathrm{\mu g} / \mathrm{ml})$ : & $<1.62 \mathrm{E}-03$ & $<1.62 \mathrm{E}-03$ & Co \\
\hline & Uncorrected weight of impurity in sample $(\mu \mathrm{g})$ : & $<0.20$ & $<0.20$ & $<0.40$ \\
\hline & Weight of impurity in blank $(\mu g)$ : & $<0.24$ & $<0.20$ & \\
\hline & Minimum corrected weight of impurity in sample $(\mathrm{\mu g})$ : & 0.00 & 0.00 & 0.00 \\
\hline & Maximum corrected weight of impurity in sample $(\mu \mathrm{g})$ : & 0.20 & 0.20 & 0.40 \\
\hline \multirow{5}{*}{$\mathrm{Ni}$} & Measured concentration of impurity in sample $(\mu \mathrm{g} / \mathrm{ml})$ : & $<8.00 \mathrm{E}-03$ & $<8.00 \mathrm{E}-03$ & $\mathrm{Ni}$ \\
\hline & Uncorrected weight of impurity in sample $(\mu q)$ : & $<0.97$ & $<0.98$ & $<1.95$ \\
\hline & Weight of impurity in blank $(\mu \mathrm{g})$ : & $<1.18$ & $<1.00$ & \\
\hline & Minimum corrected weight of impurity in sample $(\mu \mathrm{g})$ : & 0.00 & 0.00 & 0.00 \\
\hline & Maximum corrected weight of impurity in sample $(\mu q)$ : & 0.97 & 0.98 & 1.95 \\
\hline \multirow{5}{*}{$\mathbf{C a}$} & Measured concentration of impurity in sample $(\mu \mathrm{g} / \mathrm{ml})$ : & $9.23 E-01$ & $8.32 \mathrm{E}-01$ & $\mathrm{Ca}$ \\
\hline & Uncorrected weight of impurity in sample $(\mu \mathrm{g})$ : & 111.68 & 102.34 & 214.02 \\
\hline & Weight of impurity in blank $(\mu \mathrm{g})$ : & 26.31 & 125.00 & \\
\hline & Minimum corrected weight of impurity in sample $(\mu \mathrm{g})$ : & 85.37 & 0.00 & 85.37 \\
\hline & Maximum corrected weight of impurity in sample $(\mu g)$ : & 85.37 & 0.00 & 85.37 \\
\hline \multirow{5}{*}{ Al } & Measured concentration of impurity in sample $(\mu \mathrm{g} / \mathrm{ml})$ : & $4.38 \mathrm{E}-01$ & $1.65 \mathrm{E}-01$ & Al \\
\hline & Uncorrected weight of impurity in sample $(\mu \mathrm{g})$ : & 53.00 & 20.30 & 73.29 \\
\hline & Weight of impurity in blank $(\mu \mathrm{g})$ : & 1.69 & 2.19 & \\
\hline & Minimum corrected weight of impurity in sample $(\mu g)$ : & 51.31 & 18.11 & 69.42 \\
\hline & Maximum corrected weight of impurity in sample $(\mu g)$ : & 51.31 & 18.11 & 69.42 \\
\hline \multirow{5}{*}{$\mathbf{T i}$} & Measured concentration of impurity in sample $(\mu \mathrm{g} / \mathrm{ml})$ : & $2.66 \mathrm{E}-02$ & $3.30 E-02$ & $\mathrm{Ti}$ \\
\hline & Uncorrected weight of impurity in sample $(\mathrm{\mu g})$ : & 3.22 & 4.06 & 7.28 \\
\hline & Weight of impurity in blank $(\mathrm{\mu g})$ : & $<1.18$ & $<1.00$ & \\
\hline & Minimum corrected weight of impurity in sample $(\mu g)$ : & 2.04 & 3.06 & 5.10 \\
\hline & Maximum corrected weight of impurity in sample $(\mu q)$ : & 3.22 & 4.06 & 7.28 \\
\hline \multirow{5}{*}{$\mathbf{v}$} & Measured concentration of impurity in sample $(\mu \mathrm{g} / \mathrm{ml})$ : & $2.22 \mathrm{E}-01$ & $5.68 \mathrm{E}-02$ & $\mathbf{v}$ \\
\hline & Uncorrected weight of impurity in sample $(\mu g)$ : & 26.86 & 6.99 & 33.85 \\
\hline & Weight of impurity in blank $(\mu g)$ : & $<0.29$ & $<0.25$ & \\
\hline & Minimum corrected weight of impurity in sample $(\mu g)$ : & 26.57 & 6.74 & 33.30 \\
\hline & Maximum corrected weight of impurity in sample $(\mu g)$ : & 26.86 & 6.99 & 33.85 \\
\hline
\end{tabular}

Comments

Data checked against RMAL 2419 official results of analyses by FCM on 12/09/2009

Fied c. montgomeny

$1-27-2010$ 
Data Report Form DRF-26A: Measurement of U Contamination or Impurities by Deconsolidation Leach

\begin{tabular}{|c|c|}
\hline+2 & $\begin{array}{l}\text { AGR-CHAR-DAM-26 Rev. } 1 \\
\text { Fred Montoomery }\end{array}$ \\
\hline Compact lot ID: & $\begin{array}{l}\text { Hrea Montgomery } \\
\text { LEU09-OP2-Z }\end{array}$ \\
\hline Compact lot description: & AGR-2 UCO Variant, from G73]-14-93073A \\
\hline$\frac{\text { Compact ID numbers: }}{\text { DRF filename: }}$ & 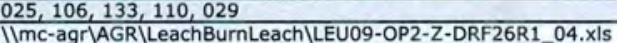 \\
\hline
\end{tabular}

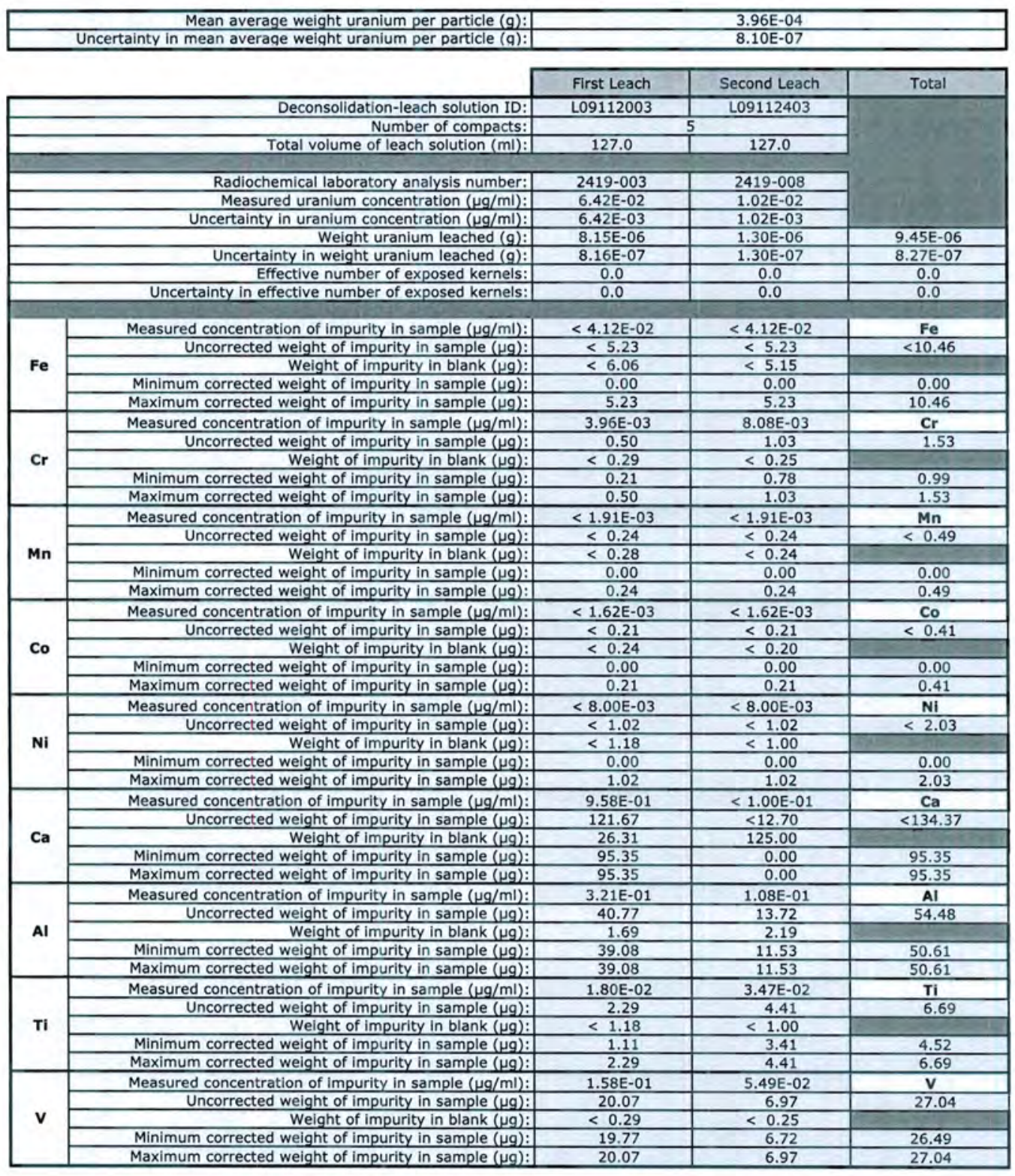

Comments

Data checked against RMAL 2419 official results of analyses by FCM on 12/09/2009

$\underset{\text { Fied c. Montgomeuy }}{\text { opertor }}$
$1-27-2010$ 


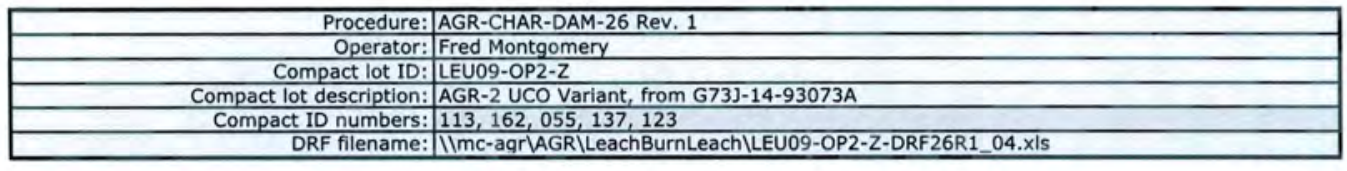

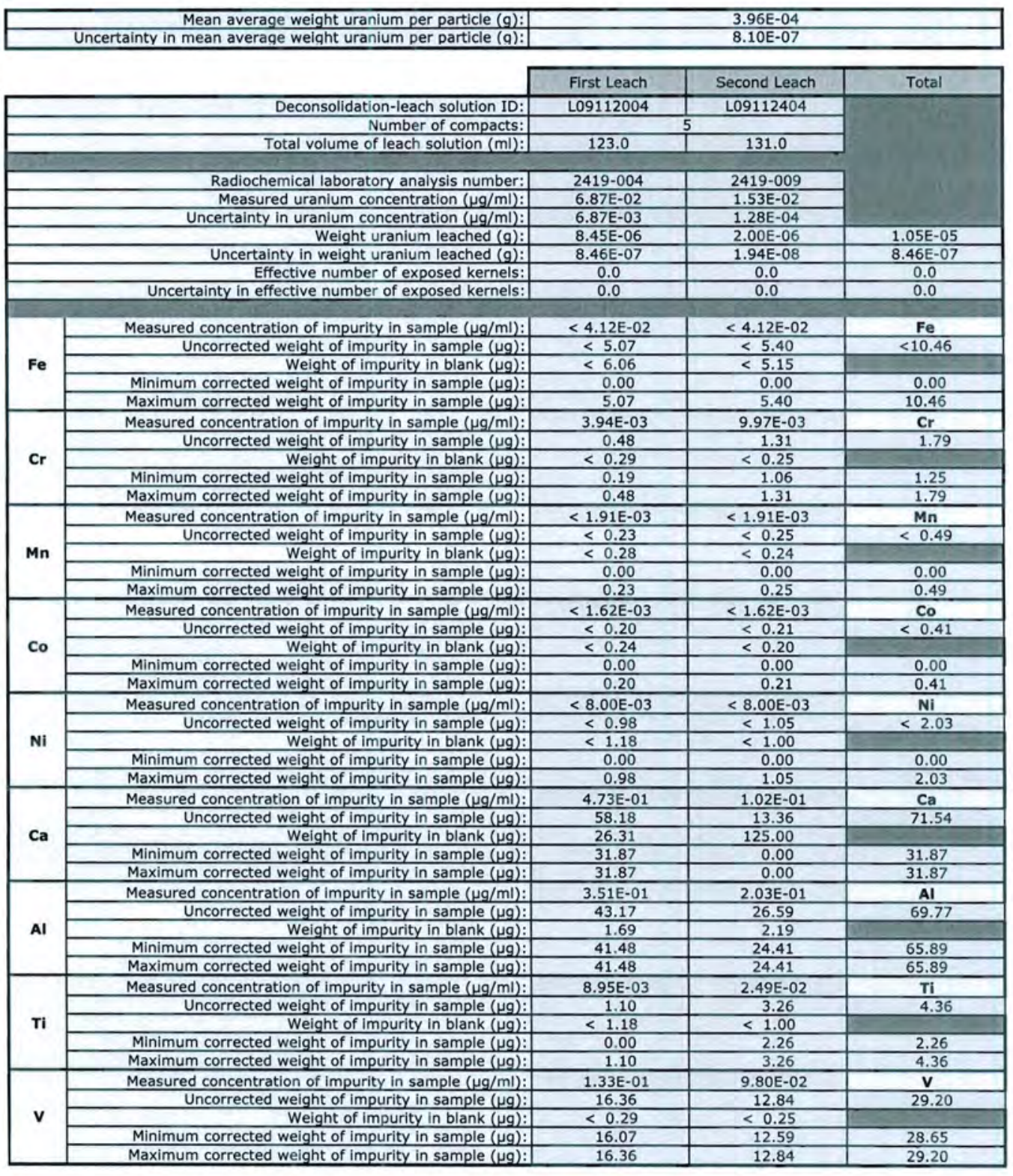

\section{Comments}

Data checked against RMAL 2419 officlal results of analyses by FCM on 12/09/2009

Fued C. Montgomey
$1-27-2010$ 
Data Report Form DRF-26A: Measurement of U Contamination or Impurities by Deconsolidation Leach

\begin{tabular}{|c|c|}
\hline Procedure: & AGR-CHAR-DAM-26 Rev. 1 \\
\hline Operator: & Fred Montgomery \\
\hline Compact lot ID: & LEU09-OP2-Z \\
\hline Compact lot description: & AGR-2 UCO Variant, from G73J-14-93073A \\
\hline Compact ID numbers: & Deconsolidation Leach Blank \\
\hline DRF filename: & |Imc-agr|AGR\LeachBurnLeach|LEU09-OP2-Z-DRF26R1_04.xls \\
\hline
\end{tabular}

\begin{tabular}{|c|c|c|c|c|}
\hline & $\begin{array}{l}\text { Mean average weight uranium per particle }(\mathrm{g}) \text { : } \\
\text { Uncertainty in mean average weight uranium per particle }(\mathrm{g}) \text { : }\end{array}$ & \multicolumn{3}{|c|}{$3.96 \mathrm{E}-04$} \\
\hline & & First Leach & Second Leach & Total \\
\hline & Deconsolidation-leach solution ID: & L09112005 & L09112405 & \\
\hline & Number of compacts: & \multicolumn{2}{|c|}{ None } & \\
\hline & Total volume of leach solution (ml): & 147.0 & 125.0 & \\
\hline & Radiochemical laboratory analysis number: & 2419-005 & $2419-010$ & \\
\hline & Measured uranium concentration $(\mu \mathrm{g} / \mathrm{ml})$ : & $<2.00 \mathrm{E}-04$ & $<2.00 \mathrm{E}-04$ & \\
\hline \multicolumn{2}{|r|}{ Uncertainty in uranium concentration $(\mu \mathrm{g} / \mathrm{ml})$ : } & & & \\
\hline & Weight uranium leached $(g)$ : & $<2.94 \mathrm{E}-08$ & $<2.50 \mathrm{E}-08$ & $<5.44 \mathrm{E}-08$ \\
\hline \multicolumn{2}{|r|}{ Uncertainty in weight uranium leached $(\mathrm{g})$ : } & & & \\
\hline \multirow{2}{*}{\multicolumn{2}{|c|}{$\begin{array}{r}\text { Effective number of exposed kernels: } \\
\text { Uncertainty in effective number of exposed kernels: }\end{array}$}} & 0.0 & 0.0 & 0.0 \\
\hline & & & & \\
\hline \multirow[b]{2}{*}{$\mathbf{F e}$} & Measured concentration $(\mu \mathrm{g} / \mathrm{ml})$ : & $<4.12 \mathrm{E}-02$ & $<4.12 \mathrm{E}-02$ & $\mathrm{Fe}$ \\
\hline & Total weight of leached impurity $(\mu \mathrm{g})$ : & $<6.06$ & $<5.15$ & $<11.21$ \\
\hline \multirow{2}{*}{$\mathbf{C r}$} & Measured concentration $(\mu \mathrm{g} / \mathrm{ml})$ : & $<2.00 \mathrm{E}-03$ & $<2.00 \mathrm{E}-03$ & $\mathrm{Cr}$ \\
\hline & Total weight of leached impurity $(\mu \mathrm{g})$ : & $<0.29$ & $<0.25$ & $<0.54$ \\
\hline \multirow{2}{*}{ Mn } & Measured concentration $(\mu \mathrm{g} / \mathrm{ml})$ : & $<1.91 \mathrm{E}-03$ & $<1.91 \mathrm{E}-03$ & Mn \\
\hline & Total weight of leached impurity $(\mu \mathrm{g})$ : & $<0.28$ & $<0.24$ & $<0.52$ \\
\hline \multirow{2}{*}{ Co } & Measured concentration $(\mu \mathrm{g} / \mathrm{ml})$ : & $<1.62 \mathrm{E}-03$ & $<1.62 \mathrm{E}-03$ & Co \\
\hline & Total weight of leached impurity $(\mu \mathrm{g})$ : & $<0.24$ & $<0.20$ & $<0.44$ \\
\hline \multirow{2}{*}{$\mathbf{N i}$} & Measured concentration $(\mu \mathrm{g} / \mathrm{ml})$ : & $<8.00 \mathrm{E}-03$ & $<8.00 \mathrm{E}-03$ & Ni \\
\hline & Total weight of leached impurity $(\mu \mathrm{g})$ : & $<1.18$ & $<1.00$ & $<2.18$ \\
\hline \multirow{2}{*}{$\mathbf{C a}$} & Measured concentration $(\mu \mathrm{g} / \mathrm{ml})$ : & $1.79 \mathrm{E}-01$ & $1.00 E+00$ & $\mathrm{Ca}$ \\
\hline & Total weight of leached impurity $(\mu \mathrm{g})$ : & 26.31 & 125.00 & 151.31 \\
\hline \multirow{2}{*}{ Al } & Measured concentration $(\mu \mathrm{g} / \mathrm{ml})$ : & $1.15 \mathrm{E}-02$ & $1.75 \mathrm{E}-02$ & Al \\
\hline & Total weight of leached impurity $(\mu \mathrm{g})$ : & 1.69 & 2.19 & 3.88 \\
\hline \multirow{2}{*}{$\mathbf{T i}$} & Measured concentration $(\mu \mathrm{g} / \mathrm{ml})$ : & $<8.00 \mathrm{E}-03$ & $<8.00 \mathrm{E}-03$ & $\mathrm{Ti}$ \\
\hline & Total weight of leached impurity $(\mu \mathrm{g})$ : & $<1.18$ & $<1.00$ & $<2.18$ \\
\hline \multirow{2}{*}{$\mathbf{v}$} & Measured concentration $(\mu \mathrm{g} / \mathrm{ml})$ : & $<2.00 \mathrm{E}-03$ & $<2.00 \mathrm{E}-03$ & v \\
\hline & Total weight of leached impurity $(\mu \mathrm{g})$ : & $<0.29$ & $<0.25$ & $<0.54$ \\
\hline
\end{tabular}

\section{Comments}

Data checked against RMAL 2419 official results of analyses by FCM on 12/09/2009

Tred c. nuntgomery
$1-27-2010$ 


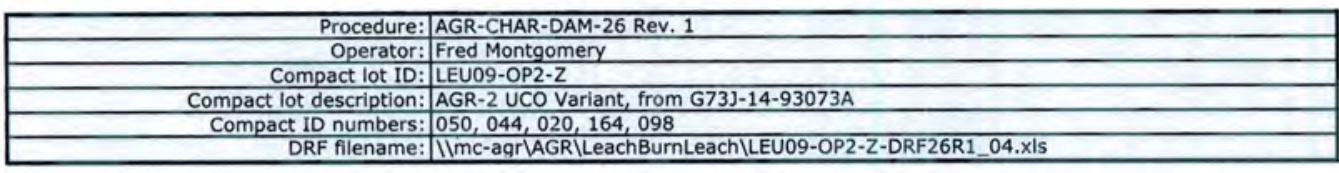

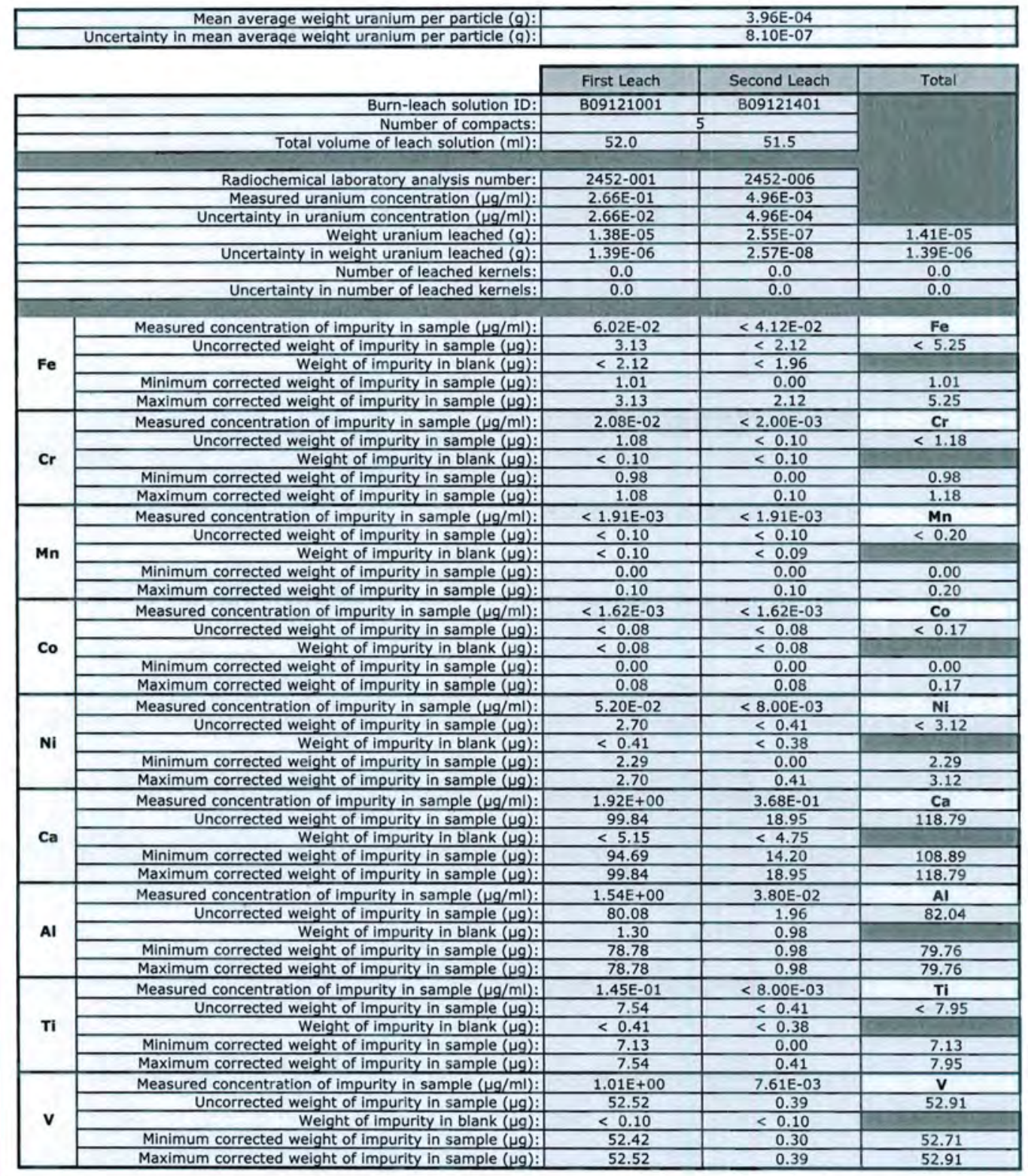

Comments

Burn-leach solution is cloudy.

Data checked against RMAL 2452 official results of analyses by FCM on 12/21/2009
Fred C. Montgo meey

$\frac{1-27-2010}{\text { Date }}$ 


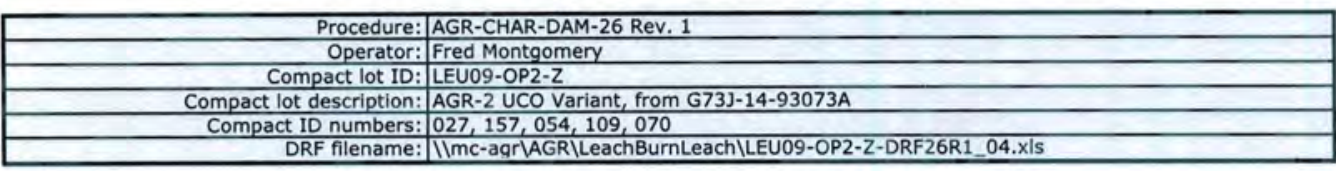

\begin{tabular}{|c|c|c|c|c|}
\hline \multirow{2}{*}{\multicolumn{2}{|c|}{$\begin{array}{r}\text { Mean average weight uranium per particle }(g): \\
\text { Uncertainty in mean average weight uranium per particle }(\mathrm{q}): \\
\end{array}$}} & \multicolumn{3}{|c|}{$3.96 \mathrm{E}-04$} \\
\hline & & \multicolumn{3}{|c|}{$8.10 \mathrm{E}-07$} \\
\hline & & First Leach & Second Leach & Total \\
\hline & Burn-leach solution ID: & B09121002 & 809121402 & \\
\hline & Number of compacts: & & & \\
\hline & Total volume of leach solution $(\mathrm{ml})$ : & 44.5 & 49.5 & \\
\hline & Radiochemical laboratory analysis number: & $2452-002$ & $2452-007$ & \\
\hline & Measured uranium concentration $(\mu \mathrm{g} / \mathrm{ml})$ : & $3.18 \mathrm{E}-01$ & $3.02 \mathrm{E}-03$ & \\
\hline & Uncertainty in uranium concentration $(\mu \mathrm{g} / \mathrm{ml})$ : & $3.18 \mathrm{E}-02$ & $3.02 E-04$ & \\
\hline & Weight uranium leached $(g):$ & $1.42 \mathrm{E}-05$ & $1.49 \mathrm{E}-07$ & $1.43 \mathrm{E}-05$ \\
\hline & Uncertainty in weight uranium leached $(\mathrm{g})$ : & $1.43 \mathrm{E}-06$ & $1.51 \mathrm{E}-08$ & $1.43 \mathrm{E}-06$ \\
\hline & Number of leached kernels: & 0.0 & 0.0 & 0.0 \\
\hline & Uncertainty in number of leached kernels: & 0.0 & 0.0 & 0.0 \\
\hline \multirow{5}{*}{$\mathrm{Fe}$} & Measured concentration of impurity in sample $(\mathrm{\mu g} / \mathrm{ml})$ : & & $<4,12 \mathrm{E}_{-}$ & $\mathrm{Fe}$ \\
\hline & $\begin{array}{r}\text { Measured concentration of impurity in sample }(\mu \mathrm{g} / \mathrm{mi}) \text { : } \\
\text { Uncorrected weight of impurity in sample }(\mu \mathrm{g}):\end{array}$ & $\frac{0.271-02}{2.79}$ & $<2.04$ & $\begin{array}{c}\mathrm{Fe} \\
<4.83\end{array}$ \\
\hline & Weight of impurity in blank $(\mu \mathrm{g})$ : & $<2.12$ & $<1.96$ & \\
\hline & Minimum corrected weight of impurity in sample $(\mu \mathrm{g})$ : & 0.67 & 0.00 & 0.67 \\
\hline & Maximum corrected weight of impurity in sample $(\mu \mathrm{g})$ : & 2.79 & 2.04 & 4.83 \\
\hline \multirow{5}{*}{$\mathbf{C r}$} & Measured concentration of impurity in sample $(\mathrm{\mu g} / \mathrm{ml})$ : & $1.97 E-02$ & $<2.00 \mathrm{E}-03$ & $\mathrm{Cr}$ \\
\hline & Uncorrected weight of impurity in sample $(\mu \mathrm{g})$ : & 0.88 & $<0.10$ & $<0.98$ \\
\hline & Weight of impurity in blank $(\mu \mathrm{g})$ : & $<0.10$ & $<0.10$ & \\
\hline & Minimum corrected weight of impurity in sample $(\mu \mathrm{g})$ : & 0.77 & 0.00 & 0.77 \\
\hline & Maximum corrected weight of impurity in sample $(\mu \mathrm{g})$ : & 0.88 & 0.10 & 0.98 \\
\hline \multirow{5}{*}{ Mn } & Measured concentration of impurity in sample $(\mu \mathrm{g} / \mathrm{ml})$ : & $<1.91 \mathrm{E}-03$ & $<1.91 \mathrm{E}-03$ & Mn \\
\hline & Uncorrected weight of impurity in sample $(\mu \mathrm{g})$ : & $<0.08$ & $<0.09$ & $<0.18$ \\
\hline & Weight of impurity in blank $(\mu \mathrm{g})$ : & $<0.10$ & $<0.09$ & \\
\hline & Minimum corrected weight of impurity in sample $(\mu \mathrm{g})$ : & 0.00 & 0.00 & 0.00 \\
\hline & Maximum corrected weight of impurity in sample $(\mu \mathrm{g})$ : & 0.08 & 0.09 & 0.18 \\
\hline \multirow{5}{*}{ Co } & Measured concentration of impurity in sample $(\mu \mathrm{g} / \mathrm{ml})$ : & $<1.62 \mathrm{E}-03$ & $<1.62 \mathrm{E}-03$ & Co \\
\hline & Uncorrected weight of impurity in sample $(\mu \mathrm{g})$ : & $<0.07$ & $<0.08$ & $<0.15$ \\
\hline & Weight of impurity in blank $(\mu \mathrm{g})$ : & $<0.08$ & $<0.08$ & \\
\hline & Minimum corrected weight of impurity in sample $(\mu \mathrm{g})$ : & 0.00 & 0.00 & 0.00 \\
\hline & Maximum corrected weight of impurity in sample $(\mu \mathrm{g})$ : & 0.07 & 0.08 & 0.15 \\
\hline \multirow{5}{*}{ Ni } & Measured concentration of impurity in sample $(\mu \mathrm{g} / \mathrm{ml})$ : & $4.33 E-02$ & $<8.00 \mathrm{E}-03$ & $\mathrm{Ni}$ \\
\hline & Uncorrected weight of impurity in sample $(\mu \mathrm{g})$ : & 1.93 & $<0.40$ & $<2.32$ \\
\hline & Weight of impurity in blank $(\mu \mathrm{g})$ : & $<0.41$ & $<0.38$ & \\
\hline & Minimum corrected weight of impurity in sample $(\mu \mathrm{g})$ : & 1.51 & 0.00 & 1.51 \\
\hline & Maximum corrected weight of impurity in sample $(\mu \mathrm{g})$ : & 1.93 & 0.40 & 2.32 \\
\hline \multirow{5}{*}{$\mathbf{C a}$} & Measured concentration of impurity in sample $(\mu \mathrm{g} / \mathrm{ml})$ : & $1.94 \mathrm{E}+00$ & $<1.00 \mathrm{E}-01$ & $\mathrm{Ca}$ \\
\hline & Uncorrected weight of impurity in sample $(\mu g)$ : & 86.33 & $<4.95$ & $<91.28$ \\
\hline & Weight of impurity in blank $(\mu \mathrm{g})$ : & $<5.15$ & $<4.75$ & \\
\hline & Minimum corrected weight of impurity in sample $(\mu \mathrm{g})$ : & 81.18 & 0.00 & 81.18 \\
\hline & Maximum corrected weight of impurity in sample $(\mu \mathrm{g})$ : & 86.33 & 4.95 & 91.28 \\
\hline \multirow{5}{*}{ Al } & Measured concentration of impurity in sample $(\mu \mathrm{g} / \mathrm{ml})$ : & $1.53 E+00$ & $2.70 E-02$ & Al \\
\hline & Uncorrected weight of impurity in sample $(\mu \mathrm{g})$ : & 68.09 & 1.34 & 69.42 \\
\hline & Weight of impurity in blank $(\mu g)$ : & 1.30 & 0.98 & \\
\hline & Minimum corrected weight of impurity in sample $(\mu \mathrm{g})$ : & 66.78 & 0.36 & 67.14 \\
\hline & Maximum corrected weight of impurity in sample $(\mu \mathrm{g})$ : & 66.78 & 0.36 & 67.14 \\
\hline \multirow{5}{*}{$\mathbf{T i}$} & Measured concentration of impurity in sample $(\mu \mathrm{g} / \mathrm{ml})$ : & $1.49 \mathrm{E}-01$ & $<8.00 \mathrm{E}-03$ & $\mathrm{Ti}$ \\
\hline & Uncorrected weight of impurity in sample $(\mu \mathrm{g})$ : & 6.63 & $<0.40$ & $<7.03$ \\
\hline & Weight of impurity in blank $(\mu \mathrm{g})$ : & $<0.41$ & $<0.38$ & \\
\hline & Minimum corrected weight of impurity in sample $(\mu \mathrm{g})$ : & 6.22 & 0.00 & 6.22 \\
\hline & Maximum corrected weight of impurity in sample $(\mu \mathrm{g})$ : & 6.63 & 0.40 & 7.03 \\
\hline \multirow{5}{*}{$\mathbf{v}$} & Measured concentration of impurity in sample $(\mu \mathrm{g} / \mathrm{ml})$ : & $1.04 E+00$ & $4.61 \mathrm{E}-03$ & $\mathbf{v}$ \\
\hline & Uncorrected weight of impurity in sample $(\mu \mathrm{g})$ : & 46.28 & 0.23 & 46.51 \\
\hline & Weight of impurity in blank $(\mu \mathrm{g})$ : & $<0.10$ & $<0.10$ & \\
\hline & Minimum corrected weight of impurity in sample $(\mu \mathrm{g})$ : & 46.18 & 0.13 & 46.31 \\
\hline & Maximum corrected weight of impurity in sample $(\mu \mathrm{g})$ : & 46.28 & 0.23 & 46.51 \\
\hline
\end{tabular}

L

\section{Feed C. Montgomeny}


Data Report Form DRF-26B: Measurement of SIC Burn-Leach Defects or Impurities by Burn-Leach

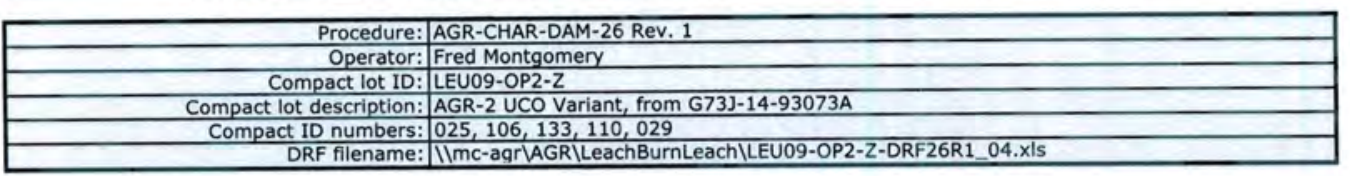

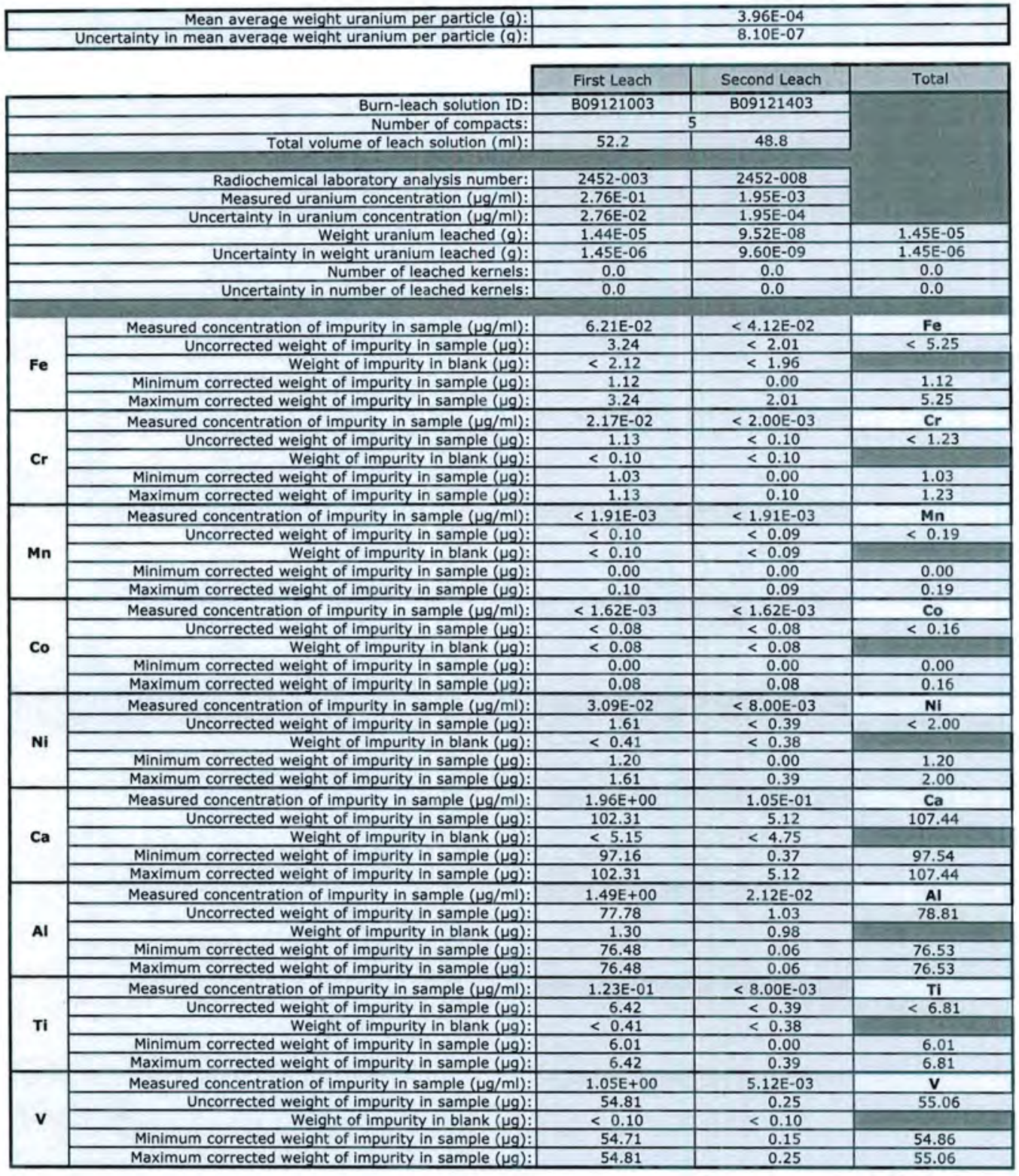

Comments

Burn-leach solution is cloudy.

Data checked against RMAL 2452 official results of analyses by FCM on 12/21/2009

\section{Feed c. Montgomery}

$1-27-2010$ 
Data Report Form DRF-26B: Measurement of SiC Burn-Leach Defects or Impurities by Burn-Leach

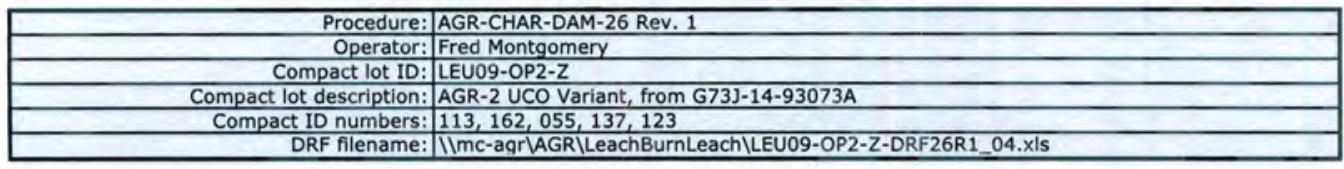

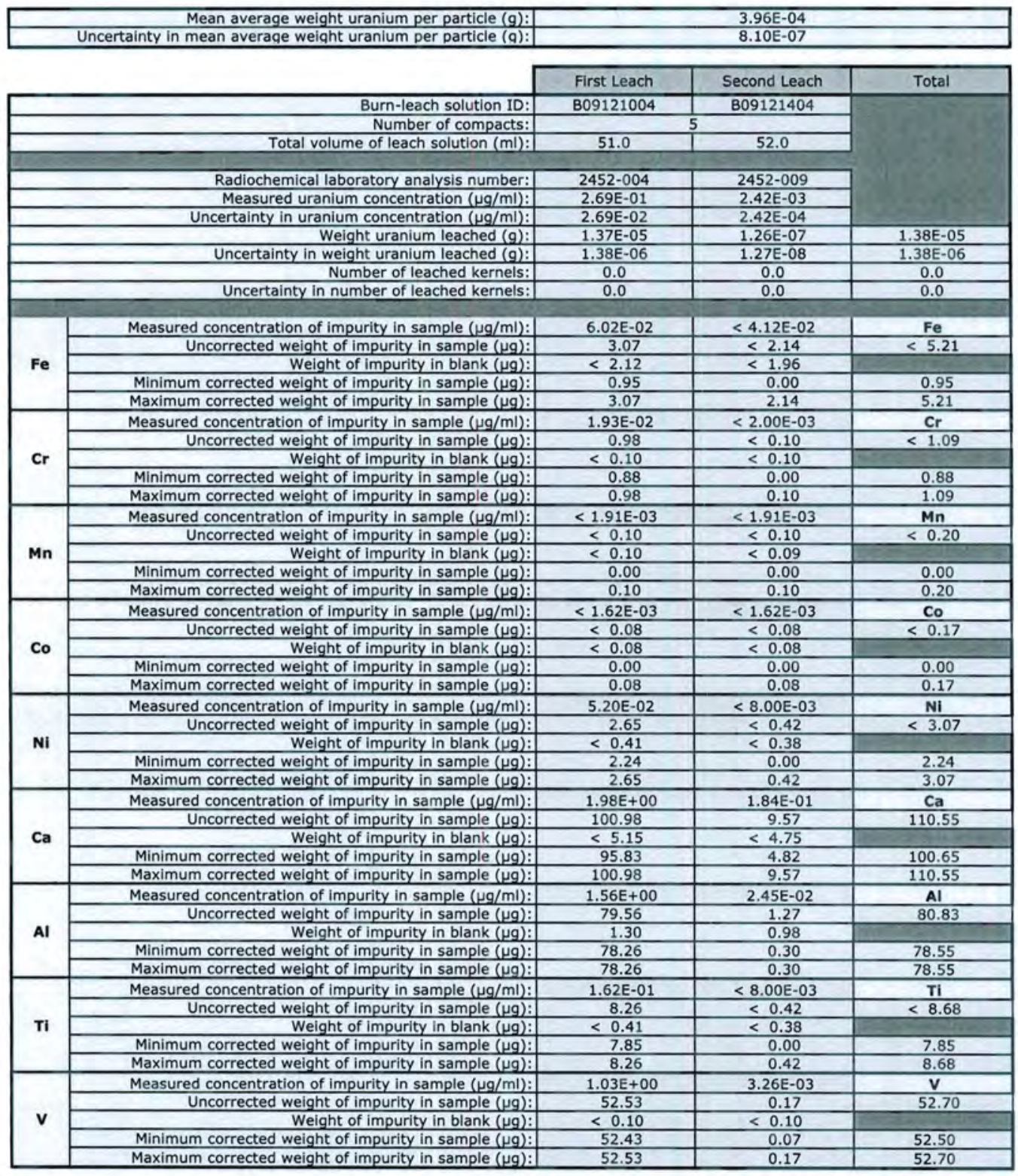

Comments

Burn-leach solution is cloudy.

Data checked against RMAL 2452 official results of analyses by FCM on 12/21/2009

Fued c. montgomery

$1-27-2010$

Date 


\section{Data Report Form DRF-26B: Measurement of SiC Burn-Leach Defects or Impurities by Burn-Leach}

\begin{tabular}{|c|c|}
\hline Procedure: & AGR-CHAR-DAM-26 Rev. 1 \\
\hline Operator: & Fred Montgomery \\
\hline Compact lot ID: & LEU09-OP2-Z \\
\hline Compact lot description: & AGR-2 UCO Variant, from G73]-14-93073A \\
\hline Compact ID numbers: & Burn-Leach Blank \\
\hline DRF filename: & IIImc-agr\AGR\LeachBurnLeach|LEU09-OP2-Z-DRF26R1_04.xIs \\
\hline
\end{tabular}

\begin{tabular}{|c|c|c|c|c|}
\hline & Mean average weight uranium per particle $(g)$ : & \multicolumn{3}{|c|}{$3.96 \mathrm{E}-04$} \\
\hline & Uncertainty in mean average weight uranium per particle $(\mathrm{g})$ : & \multicolumn{3}{|c|}{$8.10 \mathrm{E}-07$} \\
\hline & & First Leach & Second Leach & Total \\
\hline & Burn-leach solution ID: & B09121005 & B09121405 & \\
\hline & Number of compacts: & \multicolumn{2}{|c|}{ None } & \\
\hline & Total volume of leach solution $(\mathrm{ml})$ : & 51.5 & 47.5 & \\
\hline & Radiochemical laboratory analysis number: & $2452-005$ & $2452-010$ & \\
\hline & Measured uranium concentration $(\mu \mathrm{g} / \mathrm{ml})$ : & $<2.00 \mathrm{E}-04$ & $<2.00 \mathrm{E}-04$ & \\
\hline \multicolumn{5}{|c|}{ Uncertainty in uranium concentration $(\mu \mathrm{g} / \mathrm{ml}):$} \\
\hline & Weight uranium leached $(\mathrm{g})$ : & $<1.03 E-08$ & $<9.50 \mathrm{E}-09$ & $<1.98 \mathrm{E}-08$ \\
\hline \multicolumn{5}{|c|}{ Uncertainty in weight uranium leached $(\mathrm{g})$ : } \\
\hline & Number of leached kernels: & 0.0 & 0.0 & 0.0 \\
\hline \multicolumn{5}{|c|}{ Uncertainty in number of leached kernels: } \\
\hline & & & & \\
\hline \multirow{2}{*}{$\mathbf{F e}$} & Measured concentration $(\mu \mathrm{g} / \mathrm{ml})$ : & $<4.12 \mathrm{E}-02$ & $<4.12 \mathrm{E}-02$ & $\mathrm{Fe}$ \\
\hline & Total weight of leached impurity $(\mu \mathrm{g})$ : & $<2.12$ & $<1.96$ & $<4.08$ \\
\hline \multirow{2}{*}{$\mathrm{Cr}$} & Measured concentration $(\mu \mathrm{g} / \mathrm{ml})$ : & $<2.00 \mathrm{E}-03$ & $<2.00 \mathrm{E}-03$ & $\mathrm{Cr}$ \\
\hline & Total weight of leached impurity $(\mu \mathrm{g})$ : & $<0.10$ & $<0.10$ & $<0.20$ \\
\hline \multirow{2}{*}{ Mn } & Measured concentration $(\mu \mathrm{g} / \mathrm{ml})$ : & $<1.91 \mathrm{E}-03$ & $<1.91 \mathrm{E}-03$ & Mn \\
\hline & Total weight of leached impurity $(\mu \mathrm{g})$ : & $<0.10$ & $<0.09$ & $<0.19$ \\
\hline \multirow{2}{*}{ Co } & Measured concentration $(\mu \mathrm{g} / \mathrm{ml})$ : & $<1.62 \mathrm{E}-03$ & $<1.62 \mathrm{E}-03$ & Co \\
\hline & Total weight of leached impurity $(\mu \mathrm{g})$ : & $<0.08$ & $<0.08$ & $<0.16$ \\
\hline \multirow{2}{*}{$\mathbf{N i}$} & Measured concentration $(\mu \mathrm{g} / \mathrm{ml})$ : & $<8.00 \mathrm{E}-03$ & $<8.00 \mathrm{E}-03$ & Ni \\
\hline & Total weight of leached impurity $(\mu \mathrm{g})$ : & $<0.41$ & $<0.38$ & $<0.79$ \\
\hline \multirow{2}{*}{$\mathrm{Ca}$} & Measured concentration $(\mu \mathrm{g} / \mathrm{ml})$ : & $<1.00$ E- 01 & $<1.00 \mathrm{E}-01$ & $\mathrm{Ca}$ \\
\hline & Total weight of leached impurity $(\mu g)$ : & $<5.15$ & $<4.75$ & $<9.90$ \\
\hline \multirow{2}{*}{ Al } & Measured concentration $(\mu \mathrm{g} / \mathrm{ml})$ : & $2.53 \mathrm{E}-02$ & $2.06 \mathrm{E}-02$ & Al \\
\hline & Total weight of leached impurity $(\mu \mathrm{g})$ : & 1.30 & 0.98 & 2.28 \\
\hline \multirow{2}{*}{$\mathbf{T i}$} & Measured concentration $(\mu \mathrm{g} / \mathrm{ml})$ : & $<8.00 \mathrm{E}-03$ & $<8.00 \mathrm{E}-03$ & $\mathrm{Ti}$ \\
\hline & Total weight of leached impurity $(\mu \mathrm{g})$ : & $<0.41$ & $<0.38$ & $<0.79$ \\
\hline \multirow{2}{*}{ V } & Measured concentration $(\mu \mathrm{g} / \mathrm{ml})$ : & $<2.00 \mathrm{E}-03$ & $<2.00 \mathrm{E}-03$ & $\mathbf{v}$ \\
\hline & Total weight of leached impurity $(\mu g)$ : & $<0.10$ & $<0.10$ & $<0.20$ \\
\hline
\end{tabular}

Comments

Burn-leach solution is clear.

Data checked against RMAL 2452 official results of analyses by FCM on 12/21/2009

Fred c. Montomerey 
Data Report Form DRF-26A: Measurement of U Contamination or Impurities by Deconsolidation Leach

\begin{tabular}{|c|c|}
\hline \begin{tabular}{l|l} 
Procedure: \\
Operator:
\end{tabular} & $\begin{array}{l}\text { AGR-CHAR-DAM-26 Rev, } 1 \\
\text { Fred Montgomery }\end{array}$ \\
\hline Compact lot ID: & LEU09-OP2-Z \\
\hline Compact lot description: & AGR-2 UCO Variant, from G73]-14-93073A \\
\hline $\begin{aligned} \text { Compact ID numbers: } & \text { DRF filename: }\end{aligned}$ & 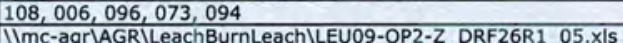 \\
\hline
\end{tabular}

\begin{tabular}{|c|c|c|c|c|}
\hline \multicolumn{2}{|r|}{$\begin{array}{l}\text { Mean average weight uranium per particle }(\mathrm{g}) \text { : } \\
\text { Uncertainty in mean average weight uranium per particle }(\mathrm{q}) \text { : }\end{array}$} & \multicolumn{3}{|c|}{$\frac{3.964 \mathrm{E}-04}{8.10 \mathrm{E}-07}$} \\
\hline & & First Leach & Second Leach & Total \\
\hline & Deconsolidation-leach solution ID: & L10010602 & L10010802 & \\
\hline & Number of compacts: & & & \\
\hline & Total volume of leach solution (ml): & 118.0 & 120.0 & \\
\hline & Radiochemical laboratory analysis number: & $2522-002$ & $2522-007$ & \\
\hline & Measured uranium concentration $(\mathrm{\mu g} / \mathrm{ml})$ : & $8.10 \mathrm{E}-02$ & $1.26 \mathrm{E}-02$ & \\
\hline & Uncertainty in uranlum concentration $(\mathrm{\mu g} / \mathrm{ml})$ : & $8.10 E-03$ & $1.26 \mathrm{E}-03$ & \\
\hline & Weight uranium leached $(g)$ : & $9.56 \mathrm{E}-06$ & $1.51 \mathrm{E}-06$ & $1.11 \mathrm{E}-05$ \\
\hline & Uncertainty in weight uranium leached $(g)$ : & $9.57 E-07$ & $1.51 \mathrm{E}-07$ & $9.69 \mathrm{E}-07$ \\
\hline & Effective number of exposed kernels: & 0.0 & 0.0 & 0.0 \\
\hline & Uncertainty in effective number of exposed kernels: & 0.0 & 0.0 & 0.0 \\
\hline \multirow{5}{*}{$\mathbf{F e}$} & Measured concentration of impurity in sample $(\mu \mathrm{g} / \mathrm{ml})$ : & $<4.12 \mathrm{E}-02$ & $<4.12 \mathrm{E}-02$ & $\mathbf{F e}$ \\
\hline & Uncorrected weight of impurity in sample $(\mu g)$ : & $<4.86$ & $<4.94$ & $<9.81$ \\
\hline & Weight of impurity in blank $(\mu g)$ : & $<6.06$ & $<5.31$ & \\
\hline & Minimum corrected weight of impurity in sample $(\mu g)$ : & 0.00 & 0.00 & 0.00 \\
\hline & Maximum corrected weight of impurity in sample $(\mu g)$ : & 4.86 & 4.94 & 9.81 \\
\hline \multirow{5}{*}{ cr } & Measured concentration of impurity in sample $(\mu \mathrm{g} / \mathrm{ml})$ : & $1.54 \mathrm{E}-02$ & 7.95E-03 & $\mathrm{Cr}$ \\
\hline & Uncorrected weight of impurity in sample $(\mu \mathrm{g})$ : & 1.82 & 0.95 & 2.77 \\
\hline & Weight of impurity in blank $(\mu g)$ : & $<0.29$ & $<0.26$ & \\
\hline & Minimum corrected weight of impurity in sample $(\mu \mathrm{g})$ : & 1.52 & 0.70 & 2.22 \\
\hline & Maximum corrected weight of impurity in sample $(\mu \mathrm{g})$ : & 1.82 & 0.95 & 2.77 \\
\hline \multirow{4}{*}{$\mathrm{Mn}$} & Measured concentration of impurity in sample $(\mu \mathrm{g} / \mathrm{ml})$ : & $<1.91 \mathrm{E}-03$ & $<1.91 \mathrm{E}-03$ & $\mathrm{Mn}$ \\
\hline & $\begin{array}{l}\text { Uncorrected weight of impurity in sample }(\mu \mathrm{g}) \text { : } \\
\text { Weight of impurity in blank }(\mu \mathrm{q}) \text { : }\end{array}$ & $<0.23$ & $<0.23$ & $<0.45$ \\
\hline & $\begin{array}{l}\text { Weight of impurity in blank }(\mu g) \text { : } \\
\text { Minimum corrected weight of impurity in sample }(\mu \mathrm{g}) \text { : }\end{array}$ & $<0.28$ & $<0.25$ & \\
\hline & $\begin{array}{l}\text { Minimum corrected weight of impurity in sample }(\mu \mathrm{g}) \text { : } \\
\text { Maximum corrected weight of impurity in sample }(\mu \mathrm{g}) \text { : }\end{array}$ & 0.00 & 0.00 & 0.00 \\
\hline \multirow{5}{*}{ co } & $\begin{array}{l}\text { Maximum corrected weight of impurity in sample }(\mu \mathrm{g}) \text { : } \\
\text { Measured concentration of impurity in sample }(\mu \mathrm{g} / \mathrm{ml})\end{array}$ & $\frac{0.23}{<1.62 \mathrm{E}-03}$ & $\leq 1.62 \mathrm{E}-03$ & $\frac{0.45}{\text { Co }}$ \\
\hline & Uncorrected weight of impurity in sample $(\mu \mathrm{g})$ : & $<0.19$ & $<0.19$ & $<0.39$ \\
\hline & Weight of impurity in blank $(\mu \mathrm{g})$ : & $<0.24$ & $<0.21$ & \\
\hline & Minimum corrected weight of impurity in sample $(\mu \mathrm{g})$ : & 0.00 & 0.00 & 0.00 \\
\hline & Maximum corrected weight of impurity in sample $(\mu \mathrm{g})$ : & 0.19 & 0.19 & 0.39 \\
\hline \multirow{5}{*}{ Ni } & Measured concentration of impurity in sample $(\mu \mathrm{g} / \mathrm{ml})$ : & $1.36 \mathrm{E}-02$ & $<8.00 \mathrm{E}-03$ & $\mathrm{Ni}$ \\
\hline & Uncorrected weight of impurity in sample $(\mu \mathrm{g})$ : & 1.60 & $<0.96$ & $<2.56$ \\
\hline & Weight of impurity in blank $(\mathrm{\mu g})$ : & $<1.18$ & $<1.03$ & \\
\hline & Minimum corrected weight of impurity in sample $(\mu \mathrm{g})$ : & 0.43 & 0.00 & 0.43 \\
\hline & Maximum corrected weight of impurity in sample $(\mu \mathrm{g})$ : & 1.60 & 0.96 & 2.56 \\
\hline \multirow{5}{*}{$\mathbf{C a}$} & Measured concentration of impurity in sample $(\mu \mathrm{g} / \mathrm{ml})$ : & $5.54 \mathrm{E}-01$ & $<1.00 \mathrm{E}-01$ & $\mathrm{Ca}$ \\
\hline & Uncorrected weight of impurity in sample $(\mu \mathrm{g})$ : & 65.37 & $<12.00$ & $<77.37$ \\
\hline & Weight of impurity in blank $(\mu g)$ : & $<14.70$ & 19.87 & \\
\hline & Minimum corrected weight of impurity in sample $(\mu \mathrm{g})$ : & 50.67 & 0.00 & 50.67 \\
\hline & Maximum corrected weight of impurity in sample $(\mu \mathrm{g})$ : & 65.37 & 0.00 & 65.37 \\
\hline \multirow{5}{*}{ Al } & Measured concentration of impurity in sample $(\mu \mathrm{g} / \mathrm{ml})$ : & $7.60 \mathrm{E}-01$ & $1.28 \mathrm{E}-01$ & Al \\
\hline & Uncorrected weight of impurity in sample $(\mu \mathrm{g})$ : & 89.68 & 15.36 & 105.04 \\
\hline & Weight of impurity in blank $(\mu \mathrm{g})$ : & 7.23 & 1.15 & \\
\hline & Minimum corrected weight of impurity in sample $(\mu \mathrm{g})$ : & 82.45 & 14.21 & 96.66 \\
\hline & Maximum corrected weight of impurity in sample $(\mathrm{\mu g})$ : & 82.45 & 14.21 & 96.66 \\
\hline \multirow{5}{*}{$\mathbf{T i}$} & Measured concentration of impurity in sample $(\mu \mathrm{g} / \mathrm{ml})$ : & $3.83 E-02$ & $3.09 \mathrm{E}-02$ & Ti \\
\hline & Uncorrected weight of impurity in sample $(\mu \mathrm{g})$ : & 4.52 & 3.71 & 8.23 \\
\hline & Weight of impurity in blank $(\mu \mathrm{g})$ : & $<1.18$ & $<1.03$ & \\
\hline & Minimum corrected weight of impurity in sample $(\mu \mathrm{g})$ : & 3.34 & 2.68 & 6.02 \\
\hline & Maximum corrected weight of impurity in sample $(\mu \mathrm{g})$ : & 4.52 & 3.71 & 8.23 \\
\hline \multirow{5}{*}{$\mathbf{v}$} & Measured concentration of impurity in sample $(\mu \mathrm{g} / \mathrm{ml})$ : & $2.38 \mathrm{E}-01$ & $\frac{4.73 E-02}{5-68}$ & 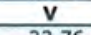 \\
\hline & Uncorrected weight of impurity in sample $(\mu \mathrm{g})$ : & 28.08 & 5.68 & 33.76 \\
\hline & Weight of impurity in blank $(\mu g)$ : & $<0.29$ & $<0.26$ & \\
\hline & Minimum corrected weight of impurity in sample $(\mu \mathrm{g})$ : & 27.79 & 5.42 & 33.21 \\
\hline & Maximum corrected weight of impurity in sample $(\mu \mathrm{g})$ : & 28.08 & 5.68 & 33.76 \\
\hline
\end{tabular}

Comments

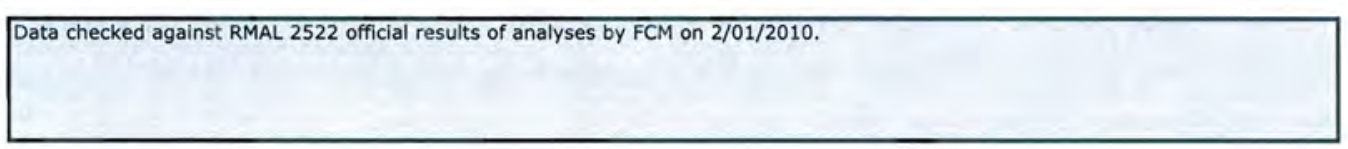

Fued c. Montgomeny

$2.11-2010$ 


\begin{tabular}{|c|c|}
\hline Procedure: & AGR-CHAR-DAM-26 Rev. 1 \\
\hline Operator: & Fred Montgomery \\
\hline Compact lot ID: & LEU09-OP2-Z \\
\hline Compact lot description: & AGR-2 UCO Variant, from G73J-14-93073A \\
\hline Compact ID numbers: & $166,111,099,042,120$ \\
\hline DRF filename: & 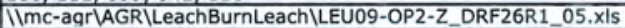 \\
\hline
\end{tabular}

\begin{tabular}{|c|c|c|c|c|}
\hline \multirow{2}{*}{\multicolumn{2}{|c|}{$\begin{array}{r}\text { Mean average weight uranium per particle }(\mathrm{g}): \\
\text { Uncertainty in mean average weight uranium per particle }(\mathrm{q}): \\
\end{array}$}} & \multirow{2}{*}{\multicolumn{3}{|c|}{$\frac{3.96 \mathrm{E}-04}{8.10 \mathrm{E}-07}$}} \\
\hline & & & & \\
\hline & & First Leach & Second Leach & Total \\
\hline & Deconsolidation-leach solution ID: & L10010603 & L10010803 & \\
\hline & Number of compacts: & & & \\
\hline & Total volume of leach solution $(\mathrm{ml})$ : & 124.0 & 125.0 & \\
\hline & 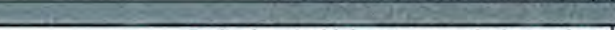 & & & \\
\hline & Radiochemical laboratory analysis number: & $2522-003$ & $2522-008$ & \\
\hline & Measured uranium concentration $(\mu \mathrm{g} / \mathrm{ml})$ : & $7.67 E-02$ & $1.29 \mathrm{E}-02$ & \\
\hline & Uncertainty in uranium concentration $(\mu \mathrm{g} / \mathrm{ml})$ : & 7.67E-03 & $1.29 \mathrm{E}-03$ & \\
\hline & Weight uranium leached $(g)$ : & $9.51 \mathrm{E}-06$ & $1.61 \mathrm{E}-06$ & $1.11 \mathrm{E}-05$ \\
\hline & Uncertainty in weight uranium leached $(9)$ : & $9.52 \mathrm{E}-07$ & $1.61 \mathrm{E}-07$ & $9.66 \mathrm{E}-07$ \\
\hline & Effective number of exposed kernels: & 0.0 & 0.0 & 0.0 \\
\hline & Uncertainty in effective number of exposed kernels: & 0.0 & 0.0 & 0.0 \\
\hline \multirow{5}{*}{$\mathbf{F e}$} & Measured concentration of impurity in sample $(\mu \mathrm{g} / \mathrm{ml})$ : & $<4.12 \mathrm{E}-02$ & $<4.12 \mathrm{E}-02$ & $\mathbf{F e}$ \\
\hline & Uncorrected weight of impurity in sample $(\mu g)$ : & $<5.11$ & $<5.15$ & $<10.26$ \\
\hline & Weight of impurity in blank $(\mu \mathrm{g})$ : & $<6.06$ & $<5.31$ & \\
\hline & Minimum corrected weight of impurity in sample $(\mu \mathrm{g})$ : & 0.00 & 0.00 & 0.00 \\
\hline & Maximum corrected weight of impurity in sample $(\mu \mathrm{g})$ : & 5.11 & 5.15 & 10.26 \\
\hline \multirow{5}{*}{$\mathbf{C r}$} & Measured concentration of impurity in sample $(\mu \mathrm{g} / \mathrm{ml})$ : & $1.48 \mathrm{E}-02$ & $7.14 \mathrm{E}-03$ & $\mathrm{Cr}$ \\
\hline & Uncorrected weight of impurity in sample $(\mu \mathrm{g})$ : & 1.84 & 0.89 & 2.73 \\
\hline & Weight of impurity in blank $(\mu \mathrm{g})$ : & $<0.29$ & $<0.26$ & \\
\hline & Minimum corrected weight of impurity in sample $(\mu \mathrm{g})$ : & 1.54 & 0.63 & 2.18 \\
\hline & Maximum corrected weight of impurity in sample $(\mu \mathrm{g})$ : & 1.84 & 0.89 & 2.73 \\
\hline \multirow{5}{*}{ Mn } & Measured concentration of impurity in sample $(\mu \mathrm{g} / \mathrm{ml})$ : & $<1.91 \mathrm{E}-03$ & $<1.91 \mathrm{E}-03$ & Mn \\
\hline & Uncorrected weight of impurity in sample $(\mu \mathrm{g})$ : & $<0.24$ & $<0.24$ & $<0.48$ \\
\hline & Weight of impurity in blank $(\mu \mathrm{g})$ : & $<0.28$ & $<0.25$ & \\
\hline & Minimum corrected weight of impurity in sample $(\mu \mathrm{g})$ : & 0.00 & 0.00 & 0.00 \\
\hline & Maximum corrected weight of impurity in sample $(\mu \mathrm{g})$ : & 0.24 & 0.24 & 0.48 \\
\hline \multirow{5}{*}{ Co } & Measured concentration of impurity in sample $(\mu \mathrm{g} / \mathrm{ml})$ : & $<1.62 \mathrm{E}-03$ & $<1.62 \mathrm{E}-03$ & Co \\
\hline & Uncorrected weight of impurity in sample $(\mu \mathrm{g})$ : & $<0.20$ & $<0.20$ & $<0.40$ \\
\hline & Weight of impurity in blank $(\mu \mathrm{g})$ : & $<0.24$ & $<0.21$ & \\
\hline & Minimum corrected weight of impurity in sample $(\mu \mathrm{g})$ : & 0.00 & 0.00 & 0.00 \\
\hline & Maximum corrected weight of impurity in sample $(\mu \mathrm{g})$ : & 0.20 & 0.20 & 0.40 \\
\hline \multirow{5}{*}{ Ni } & Measured concentration of impurity in sample $(\mu \mathrm{g} / \mathrm{ml})$ : & $<8.00 \mathrm{E}-03$ & $<8.00 \mathrm{E}-03$ & $\mathrm{Ni}$ \\
\hline & Uncorrected weight of impurity in sample $(\mu \mathrm{g})$ : & $<0.99$ & $<1.00$ & $<1.99$ \\
\hline & Weight of impurity in blank $(\mu \mathrm{g})$ : & $<1.18$ & $<1.03$ & \\
\hline & Minimum corrected weight of impurity in sample $(\mu \mathrm{g})$ : & 0.00 & 0.00 & 0.00 \\
\hline & Maximum corrected weight of impurity in sample $(\mu \mathrm{g})$ : & 0.99 & 1.00 & 1.99 \\
\hline \multirow{5}{*}{$\mathbf{C a}$} & Measured concentration of impurity in sample $(\mu \mathrm{g} / \mathrm{ml})$ : & $3.26 \mathrm{E}-01$ & $1.92 \mathrm{E}-01$ & Ca \\
\hline & Uncorrected weight of impurity in sample $(\mu \mathrm{g})$ : & 40.42 & 24.00 & 64.42 \\
\hline & Weight of impurity in blank $(\mu g)$ : & $<14.70$ & 19.87 & \\
\hline & Minimum corrected weight of impurity in sample $(\mu \mathrm{g})$ : & 25.72 & 4.13 & 29.86 \\
\hline & Maximum corrected weight of impurity in sample $(\mu \mathrm{g})$ : & 40.42 & 4.13 & 44.56 \\
\hline \multirow{5}{*}{ Al } & Measured concentration of impurity in sample $(\mu \mathrm{g} / \mathrm{ml})$ : & $4.82 \mathrm{E}-01$ & $1.23 E-01$ & Al \\
\hline & Uncorrected weight of impurity in sample $(\mu \mathrm{g})$ : & 59.77 & 15.38 & 75.14 \\
\hline & Weight of impurity in blank $(\mu \mathrm{g})$ : & 7.23 & 1.15 & \\
\hline & Minimum corrected weight of impurity in sample $(\mu \mathrm{g})$ : & 52.54 & 14.22 & 66.76 \\
\hline & Maximum corrected weight of impurity in sample $(\mu \mathrm{g})$ : & 52.54 & 14.22 & 66.76 \\
\hline \multirow{5}{*}{$\mathbf{T i}$} & Measured concentration of impurity in sample $(\mu \mathrm{g} / \mathrm{ml})$ : & 3.77E-02 & $2.74 E-02$ & $\mathbf{T i}$ \\
\hline & Uncorrected weight of impurity in sample $(\mu \mathrm{g})$ : & 4.67 & 3.43 & 8.10 \\
\hline & Weight of impurity in blank $(\mu \mathrm{g})$ : & $<1.18$ & $<1.03$ & \\
\hline & Minimum corrected weight of impurity in sample $(\mu \mathrm{g})$ : & 3.50 & 2.39 & 5.89 \\
\hline & Maximum corrected weight of impurity in sample $(\mu \mathrm{g})$ : & 4.67 & 3.43 & 8.10 \\
\hline \multirow{5}{*}{$\mathbf{v}$} & Measured concentration of impurity in sample $(\mu \mathrm{g} / \mathrm{ml})$ : & $2.30 \mathrm{E}-01$ & $4.82 \mathrm{E}-02$ & $\mathbf{v}$ \\
\hline & Uncorrected weight of impurity in sample $(\mu \mathrm{g})$ : & 28.52 & 6.03 & 34.55 \\
\hline & Weight of impurity in blank $(\mu \mathrm{g})$ : & $<0.29$ & $<0.26$ & \\
\hline & Minimum corrected weight of impurity in sample $(\mu \mathrm{g})$ : & 28.23 & 5.77 & 33.99 \\
\hline & Maximum corrected weight of impurity in sample $(\mu \mathrm{g})$ : & 28.52 & 6.03 & 34.55 \\
\hline
\end{tabular}

\section{Comments}

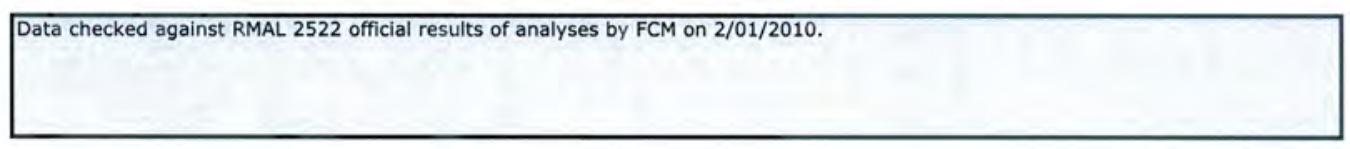

\section{Fud c. montromeney

$2-11-2010$ 


\begin{tabular}{|r|l|}
\hline Procedure: & AGR-CHAR-DAM-26 Rev. 1 \\
\hline Operator: & Fred Montgomery \\
\hline Compact lot ID: & LEU09-OP2-Z \\
\hline Compact lot description: & AGR-2 UCO Variant, from G73J-14-93073A \\
\hline Compact ID numbers: & $101,078,136,087,122$ \\
\hline DRF filename: & IImC-aqrVAGR\LeachBurnLeach \LEU09-OP2-Z_DRF26R1_05.xIs \\
\hline
\end{tabular}

\begin{tabular}{|c|c|c|c|c|}
\hline \multirow{2}{*}{\multicolumn{2}{|c|}{$\begin{array}{r}\text { Mean average weight uranium per particle }(g): \\
\text { Uncertainty in mean average weight uranium per particle }(\mathrm{q}): \\
\end{array}$}} & \multicolumn{3}{|c|}{$3.96 \mathrm{E}-04$} \\
\hline & & \multicolumn{3}{|c|}{$8.10 \mathrm{E}-07$} \\
\hline & & First Leach & Second Leach & Total \\
\hline & Deconsolidation-leach solution ID: & L10010604 & L10010804 & \\
\hline & Number of compacts: & & & \\
\hline & Total volume of leach solution $(\mathrm{ml})$ : & 121.0 & 122.0 & \\
\hline & 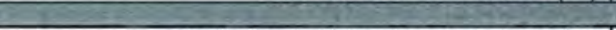 & 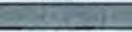 & & \\
\hline & Radiochemical laboratory analysis number: & $2522-004$ & $2522-009$ & \\
\hline & Measured uranium concentration $(\mu \mathrm{g} / \mathrm{ml})$ : & 7.57E-02 & $1.17 E-02$ & \\
\hline & Uncertainty in uranium concentration $(\mu \mathrm{g} / \mathrm{ml})$ : & 7.57E-03 & 1.17E-03 & \\
\hline & Weight uranium leached $(g):$ & $9.16 \mathrm{E}-06$ & $1.43 \mathrm{E}-06$ & $1.06 \mathrm{E}-05$ \\
\hline & Uncertainty in weight uranium leached $(\mathrm{g})$ : & $9.17 \mathrm{E}-07$ & $1.43 \mathrm{E}-07$ & $9.28 \mathrm{E}-07$ \\
\hline & Effective number of exposed kernels: & 0.0 & 0.0 & 0.0 \\
\hline & Uncertainty in effective number of exposed kernels: & 0.0 & 0.0 & 0.0 \\
\hline & & & & \\
\hline \multirow{5}{*}{$\mathbf{F e}$} & Measured concentration of impurity in sample $(\mu \mathrm{g} / \mathrm{ml})$ : & $<4.12 \mathrm{E}-02$ & $<4.12 \mathrm{E}-02$ & $\mathrm{Fe}$ \\
\hline & Uncorrected weight of impurity in sample $(\mu \mathrm{g})$ : & $<4.99$ & $<5.03$ & $<10.01$ \\
\hline & Weight of impurity in blank $(\mu \mathrm{g})$ : & $<6.06$ & $<5.31$ & \\
\hline & Minimum corrected weight of impurity in sample $(\mu \mathrm{g})$ : & 0.00 & 0.00 & 0.00 \\
\hline & Maximum corrected weight of impurity in sample $(\mu \mathrm{g})$ : & 4.99 & 5.03 & 10.01 \\
\hline \multirow{5}{*}{$\mathbf{C r}$} & Measured concentration of impurity in sample $(\mu \mathrm{g} / \mathrm{ml})$ : & $1.37 \mathrm{E}-02$ & 7.84E-03 & $\mathrm{Cr}$ \\
\hline & Uncorrected weight of impurity in sample $(\mu \mathrm{g})$ : & 1.66 & 0.96 & 2.61 \\
\hline & Weight of impurity in blank $(\mu \mathrm{g})$ : & $<0.29$ & $<0.26$ & \\
\hline & Minimum corrected weight of impurity in sample $(\mu \mathrm{g})$ : & 1.36 & 0.70 & 2.06 \\
\hline & Maximum corrected weight of impurity in sample $(\mu \mathrm{g})$ : & 1.66 & 0.96 & 2.61 \\
\hline \multirow{5}{*}{ Mn } & Measured concentration of impurity in sample $(\mu \mathrm{g} / \mathrm{ml})$ : & $<1.91 \mathrm{E}-03$ & $<1.91 \mathrm{E}-03$ & Mn \\
\hline & Uncorrected weight of impurity in sample $(\mu \mathrm{g})$ : & $<0.23$ & $<0.23$ & $<0.46$ \\
\hline & Weight of impurity in blank $(\mu \mathrm{g})$ : & $<0.28$ & $<0.25$ & \\
\hline & Minimum corrected weight of impurity in sample $(\mu \mathrm{g})$ : & 0.00 & 0.00 & 0.00 \\
\hline & Maximum corrected weight of impurity in sample $(\mu \mathrm{g})$ : & 0.23 & 0.23 & 0.46 \\
\hline \multirow{5}{*}{ Co } & Measured concentration of impurity in sample $(\mu \mathrm{g} / \mathrm{ml})$ : & $<1.62 \mathrm{E}-03$ & $<1.62 \mathrm{E}-03$ & Co \\
\hline & Uncorrected weight of impurity in sample $(\mu \mathrm{g})$ : & $<0.20$ & $<0.20$ & $<0.39$ \\
\hline & Weight of impurity in blank $(\mu \mathrm{g})$ : & $<0.24$ & $<0.21$ & \\
\hline & Minimum corrected weight of impurity in sample $(\mu \mathrm{g})$ : & 0.00 & 0.00 & 0.00 \\
\hline & Maximum corrected weight of Impurity in sample $(\mu \mathrm{g})$ : & 0.20 & 0.20 & 0.39 \\
\hline \multirow{5}{*}{$\mathbf{N i}$} & Measured concentration of impurity in sample $(\mu \mathrm{g} / \mathrm{ml})$ : & $<8.00 \mathrm{E}-03$ & $<8.00 \mathrm{E}-03$ & $\mathrm{Ni}$ \\
\hline & Uncorrected weight of impurity in sample $(\mu \mathrm{g})$ : & $<0.97$ & $<0.98$ & $<1.94$ \\
\hline & Weight of impurity in blank $(\mu \mathrm{g})$ : & $<1.18$ & $<1.03$ & \\
\hline & Minimum corrected weight of impurity in sample $(\mu \mathrm{g})$ : & 0.00 & 0.00 & 0.00 \\
\hline & Maximum corrected weight of impurity in sample $(\mu \mathrm{g})$ : & 0.97 & 0.98 & 1.94 \\
\hline \multirow{5}{*}{$\mathbf{C a}$} & Measured concentration of impurity in sample $(\mu \mathrm{g} / \mathrm{ml})$ : & 3.81E-01 & $5.54 \mathrm{E}-01$ & $\mathrm{Ca}$ \\
\hline & Uncorrected weight of impurity in sample $(\mu \mathrm{g})$ : & 46.10 & 67.59 & 113.69 \\
\hline & Weight of impurity in blank $(\mu \mathrm{g})$ : & $<14.70$ & 19.87 & \\
\hline & Minimum corrected weight of Impurity in sample $(\mu \mathrm{g})$ : & 31.40 & 47.72 & 79.12 \\
\hline & Maximum corrected weight of impurity in sample $(\mu g)$ : & 46.10 & 47.72 & 93.82 \\
\hline \multirow{5}{*}{ Al } & Measured concentration of impurity in sample $(\mu \mathrm{g} / \mathrm{ml})$ : & 4.64E-01 & 1.05E-01 & Al \\
\hline & Uncorrected weight of impurity in sample $(\mu \mathrm{g})$ : & 56.14 & 12.81 & 68.95 \\
\hline & Weight of impurity in blank $(\mu \mathrm{g})$ : & 7.23 & 1.15 & \\
\hline & Minimum corrected weight of impurity in sample $(\mu \mathrm{g})$ : & 48.91 & 11.66 & 60.57 \\
\hline & Maximum corrected weight of impurity in sample $(\mu \mathrm{g})$ : & 48.91 & 11.66 & 60.57 \\
\hline \multirow{5}{*}{$\mathbf{T i}$} & Measured concentration of impurity in sample $(\mu \mathrm{g} / \mathrm{ml})$ : & $3.35 \mathrm{E}-02$ & $3.32 E-02$ & $\mathbf{T i}$ \\
\hline & Uncorrected weight of impurity in sample $(\mu \mathrm{g})$ : & 4.05 & 4.05 & 8.10 \\
\hline & Weight of impurity in blank $(\mu \mathrm{g})$ : & $<1.18$ & $<1.03$ & \\
\hline & Minimum corrected weight of impurity in sample $(\mu \mathrm{g})$ : & 2.88 & 3.02 & 5.90 \\
\hline & Maximum corrected weight of impurity in sample $(\mu \mathrm{g})$ : & 4.05 & 4.05 & 8.10 \\
\hline \multirow{5}{*}{$\mathbf{v}$} & Measured concentration of impurity in sample $(\mu \mathrm{g} / \mathrm{ml})$ : & 2.35E-01 & $5.12 E-02$ & $\mathbf{v}$ \\
\hline & Uncorrected weight of impurity in sample $(\mu \mathrm{g})$ : & 28.44 & 6.25 & 34.68 \\
\hline & Weight of impurity in blank $(\mu \mathrm{g})$ : & $<0.29$ & $<0.26$ & \\
\hline & Minimum corrected weight of impurity in sample $(\mu \mathrm{g})$ : & 28.14 & 5.99 & 34.13 \\
\hline & Maximum corrected weight of impurity in sample $(\mu \mathrm{g})$ : & 28.44 & 6.25 & 34.68 \\
\hline
\end{tabular}

\section{Comments}

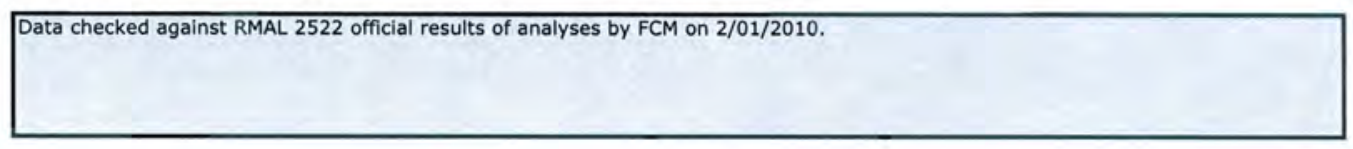

\section{Fied c. montgo meny}

$2-11.2010$ 


\begin{tabular}{|r|r|}
\hline Procedure: & AGR-CHAR-DAM-26 Rev. 1 \\
\hline Operator: & Fred Montgomery \\
\hline Compact lot ID: & LEU09-OP2-Z \\
\hline Compact lot description: & AGR-2 UCO Variant, from G73J-14-93073A \\
\hline Compact ID numbers: & $052,146,161,115,095$ \\
\hline DRF filename: & IImC-agr|AGRILeachBurnLeach \LEU09-OP2-Z_DRF26R1_05.xIs \\
\hline
\end{tabular}

\begin{tabular}{|c|c|c|c|c|}
\hline \multirow{2}{*}{\multicolumn{2}{|c|}{$\begin{array}{r}\text { Mean average weight uranium per particle }(\mathrm{g}): \\
\text { Uncertainty in mean averaqe weight uranium per particle }(\mathrm{q}): \\
\end{array}$}} & \multirow{2}{*}{\multicolumn{3}{|c|}{$\begin{array}{l}3.96 \mathrm{E}-04 \\
8.10 \mathrm{E}-07\end{array}$}} \\
\hline & & & & \\
\hline & & First Leach & Second Leach & Total \\
\hline & Deconsolidation-leach solution ID: & L10010605 & L10010805 & \\
\hline & Number of compacts: & & & \\
\hline & Total volume of leach solution $(\mathrm{ml})$ : & 122.0 & 125.0 & \\
\hline & 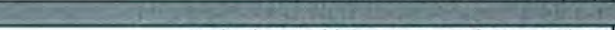 & +2 & & \\
\hline & Radiochemical laboratory analysis number: & $2522-005$ & $2522-010$ & \\
\hline & Measured uranium concentration $(\mu \mathrm{g} / \mathrm{ml})$ : & $7.67 E-02$ & $1.24 \mathrm{E}-02$ & \\
\hline & Uncertainty in uranium concentration $(\mu \mathrm{g} / \mathrm{ml})$ : & 7.67E-03 & $1.24 \mathrm{E}-03$ & \\
\hline & Weight uranium leached $(\mathrm{g})$ : & $9.36 \mathrm{E}-06$ & $1.55 \mathrm{E}-06$ & 1.09E-05 \\
\hline & Uncertainty in weight uranium leached (g): & $9.37 E-07$ & $1.55 \mathrm{E}-07$ & $9.50 \mathrm{E}-07$ \\
\hline & Effective number of exposed kernels: & 0.0 & 0.0 & 0.0 \\
\hline & Uncertainty in effective number of exposed kernels: & 0.0 & 0.0 & 0.0 \\
\hline \multirow{5}{*}{$\mathbf{F e}$} & Measured concentration of impurity in sample $(\mu \mathrm{g} / \mathrm{ml})$ : & $<4.12 \mathrm{E}-02$ & $<4.12 \mathrm{E}-02$ & $\mathbf{F e}$ \\
\hline & Uncorrected weight of impurity in sample $(\mu \mathrm{g})$ : & $<5.03$ & $<5.15$ & $<10.18$ \\
\hline & Weight of impurity in blank $(\mu \mathrm{g})$ : & $<6.06$ & $<5.31$ & \\
\hline & Minimum corrected weight of impurity in sample $(\mu \mathrm{g})$ : & 0.00 & 0.00 & 0.00 \\
\hline & Maximum corrected weight of impurity in sample $(\mu \mathrm{g})$ : & 5.03 & 5.15 & 10.18 \\
\hline \multirow{5}{*}{$\mathbf{C r}$} & Measured concentration of impurity in sample $(\mu \mathrm{g} / \mathrm{ml})$ : & $1.53 \mathrm{E}-02$ & $7.02 \mathrm{E}-03$ & $\mathrm{Cr}$ \\
\hline & Uncorrected weight of impurity in sample $(\mu \mathrm{g})$ : & 1.87 & 0.88 & 2.74 \\
\hline & Weight of impurity in blank $(\mu \mathrm{g})$ : & $<0.29$ & $<0.26$ & \\
\hline & Minimum corrected weight of impurity in sample $(\mu \mathrm{g})$ : & 1.57 & 0.62 & 2.19 \\
\hline & Maximum corrected weight of impurity in sample $(\mu \mathrm{g})$ : & 1.87 & 0.88 & 2.74 \\
\hline \multirow{5}{*}{ Mn } & Measured concentration of impurity in sample $(\mu \mathrm{g} / \mathrm{ml})$ : & $<1.91 \mathrm{E}-03$ & $<1.91 \mathrm{E}-03$ & Mn \\
\hline & Uncorrected weight of impurity in sample $(\mu \mathrm{g})$ : & $<0.23$ & $<0.24$ & $<0.47$ \\
\hline & Weight of impurity in blank $(\mu \mathrm{g})$ : & $<0.28$ & $<0.25$ & +2 \\
\hline & Minimum corrected weight of impurity in sample $(\mu \mathrm{g})$ : & 0.00 & 0.00 & 0.00 \\
\hline & Maximum corrected weight of impurity in sample $(\mu \mathrm{g})$ : & 0.23 & 0.24 & 0.47 \\
\hline \multirow{5}{*}{ Co } & Measured concentration of impurity in sample $(\mu \mathrm{g} / \mathrm{ml})$ : & $<1.62 \mathrm{E}-03$ & $<1.62 \mathrm{E}-03$ & Co \\
\hline & Uncorrected weight of impurity in sample $(\mu \mathrm{g})$ : & $<0.20$ & $<0.20$ & $<0.40$ \\
\hline & Weight of impurity in blank $(\mu \mathrm{g})$ : & $<0.24$ & $<0.21$ & \\
\hline & Minimum corrected weight of impurity in sample $(\mu \mathrm{g})$ : & 0.00 & 0.00 & 0.00 \\
\hline & Maximum corrected weight of impurity in sample $(\mu \mathrm{g})$ : & 0.20 & 0.20 & 0.40 \\
\hline \multirow{5}{*}{$\mathbf{N i}$} & Measured concentration of impurity in sample $(\mu \mathrm{g} / \mathrm{ml})$ : & $<8.00 \mathrm{E}-03$ & $<8.00 \mathrm{E}-03$ & $\mathrm{Ni}$ \\
\hline & Uncorrected weight of impurity in sample $(\mu \mathrm{g})$ : & $<0.98$ & $<1.00$ & $<1.98$ \\
\hline & Weight of impurity in blank $(\mu \mathrm{g})$ : & $<1.18$ & $<1.03$ & \\
\hline & Minimum corrected weight of impurity in sample $(\mu \mathrm{g})$ : & 0.00 & 0.00 & 0.00 \\
\hline & Maximum corrected weight of impurity in sample $(\mu \mathrm{g})$ : & 0.98 & 1.00 & 1.98 \\
\hline \multirow{5}{*}{$\mathbf{C a}$} & Measured concentration of impurity in sample $(\mu \mathrm{g} / \mathrm{ml})$ : & $1.08 E+00$ & $1.39 \mathrm{E}-01$ & $\mathbf{C a}$ \\
\hline & Uncorrected weight of impurity in sample $(\mu \mathrm{g})$ : & 131.76 & 17.38 & 149.14 \\
\hline & Weight of impurity in blank $(\mu \mathrm{g})$ : & $<14.70$ & 19.87 & \\
\hline & Minimum corrected weight of impurity in sample $(\mu \mathrm{g})$ : & 117.06 & 0.00 & 117.06 \\
\hline & Maximum corrected weight of impurity in sample $(\mu \mathrm{g})$ : & 131.76 & 0.00 & 131.76 \\
\hline \multirow{5}{*}{ Al } & Measured concentration of impurity in sample $(\mu \mathrm{g} / \mathrm{ml})$ : & $5.22 \mathrm{E}-01$ & 1.20E-01 & Al \\
\hline & Uncorrected weight of impurity in sample $(\mu \mathrm{g})$ : & 63.68 & 15.00 & 78.68 \\
\hline & Weight of impurity in blank $(\mu \mathrm{g})$ : & 7.23 & 1.15 & \\
\hline & Minimum corrected weight of impurity in sample $(\mu \mathrm{g})$ : & 56.45 & 13.85 & 70.30 \\
\hline & Maximum corrected weight of impurity in sample $(\mu \mathrm{g})$ : & 56.45 & 13.85 & 70.30 \\
\hline \multirow{5}{*}{$\mathbf{T i}$} & Measured concentration of impurity in sample $(\mu \mathrm{g} / \mathrm{ml})$ : & 4.16E-02 & 2.65E-02 & $\mathbf{T i}$ \\
\hline & Uncorrected weight of impurity in sample $(\mu \mathrm{g})$ : & 5.08 & 3.31 & 8.39 \\
\hline & Weight of impurity in blank $(\mu \mathrm{g})$ : & $<1.18$ & $<1.03$ & + \\
\hline & Minimum corrected weight of impurity in sample $(\mu \mathrm{g})$ : & 3.90 & 2.28 & 6.18 \\
\hline & Maximum corrected weight of impurity in sample $(\mu \mathrm{g})$ : & 5.08 & 3.31 & 8.39 \\
\hline \multirow{5}{*}{$\mathbf{v}$} & Measured concentration of impurity in sample $(\mu \mathrm{g} / \mathrm{ml})$ : & $2.20 \mathrm{E}-01$ & $4.34 \mathrm{E}-02$ & $\mathbf{v}$ \\
\hline & Uncorrected weight of impurity in sample $(\mu \mathrm{g})$ : & 26.84 & 5.43 & 32.27 \\
\hline & Weight of impurity in blank $(\mu \mathrm{g})$ : & $<0.29$ & $<0.26$ & $\overline{\mathrm{C}}$ \\
\hline & Minimum corrected weight of impurity in sample $(\mu \mathrm{g})$ : & 26.55 & 5.17 & 31.71 \\
\hline & Maximum corrected weight of impurity in sample $(\mu \mathrm{g})$ : & 26.84 & 5.43 & 32.27 \\
\hline
\end{tabular}

Comments

Data checked against RMAL 2522 official results of analyses by FCM on 2/01/2010.

Fied c. Montgomey $\frac{2-11-2010}{\text { operator }}$ 
Data Report Form DRF-26A: Measurement of U Contamination or Impurities by Deconsolidation Leach

\begin{tabular}{|c|c|}
\hline Procedure:| & AGR-CHAR-DAM-26 Rev. 1 \\
\hline Operator: & Fred Montgomery \\
\hline Compact lot ID: & LEU09-OP2-Z \\
\hline Compact lot description: & AGR-2 UCO Variant, from G73J-14-93073A \\
\hline Compact ID numbers: & Deconsolidation Leach Blank \\
\hline DRF filename: & 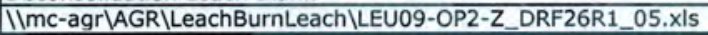 \\
\hline
\end{tabular}

\begin{tabular}{|c|c|c|c|c|}
\hline \multirow{2}{*}{\multicolumn{2}{|c|}{$\begin{array}{r}\text { Mean average weight uranium per particle }(g): \\
\text { Uncertainty in mean average weight uranium per particle }(g): \\
\end{array}$}} & \multicolumn{3}{|c|}{$3.96 \mathrm{E}-04$} \\
\hline & & \multicolumn{3}{|c|}{$8.10 \mathrm{E}-07$} \\
\hline & & First Leach & Second Leach & Total \\
\hline & Deconsolidation-leach solution ID: & L10010601 & L10010801 & \\
\hline & Number of compacts: & \multicolumn{2}{|c|}{ None } & \\
\hline & Total volume of leach solution $(\mathrm{ml})$ : & 147.0 & 129.0 & \\
\hline & Radiochemical laboratory analysis number: & $2522-001$ & $2522-006$ & \\
\hline & Measured uranium concentration $(\mu \mathrm{g} / \mathrm{ml})$ : & $<2.00 \mathrm{E}-04$ & $<2.00 \mathrm{E}-04$ & \\
\hline \multicolumn{2}{|r|}{ Uncertainty in uranium concentration $(\mu \mathrm{g} / \mathrm{ml})$ : } & & & \\
\hline & Weight uranium leached $(g)$ : & $<2.94 \mathrm{E}-08$ & $<2.58 \mathrm{E}-08$ & $<5.52 \mathrm{E}-08$ \\
\hline \multicolumn{2}{|r|}{ Uncertainty in weight uranium leached $(\mathrm{g})$ : } & & & \\
\hline \multirow{2}{*}{\multicolumn{2}{|c|}{$\begin{array}{r}\text { Effective number of exposed kernels: } \\
\text { Uncertainty in effective number of exposed kernels: }\end{array}$}} & 0.0 & 0.0 & 0.0 \\
\hline & & & & \\
\hline \multirow{2}{*}{$\mathbf{F e}$} & Measured concentration $(\mu \mathrm{g} / \mathrm{ml})$ : & $<4.12 \mathrm{E}-02$ & $<4.12 \mathrm{E}-02$ & $\mathbf{F e}$ \\
\hline & Total weight of leached impurity $(\mu \mathrm{g})$ : & $<6.06$ & $<5.31$ & $<11.37$ \\
\hline \multirow{2}{*}{$\mathbf{C r}$} & Measured concentration $(\mu \mathrm{g} / \mathrm{ml})$ : & $<2.00 \mathrm{E}-03$ & $<2.00 \mathrm{E}-03$ & $\mathrm{Cr}$ \\
\hline & Total weight of leached impurity $(\mu \mathrm{g}):$ & $<0.29$ & $<0.26$ & $<0.55$ \\
\hline \multirow{2}{*}{ Mn } & Measured concentration $(\mu \mathrm{g} / \mathrm{ml})$ : & $<1.91 \mathrm{E}-03$ & $<1.91 \mathrm{E}-03$ & Mn \\
\hline & Total weight of leached impurity $(\mu \mathrm{g})$ : & $<0.28$ & $<0.25$ & $<0.53$ \\
\hline \multirow{2}{*}{ Co } & Measured concentration $(\mu \mathrm{g} / \mathrm{ml}):$ & $<1.62 \mathrm{E}-03$ & $<1.62 \mathrm{E}-03$ & Co \\
\hline & Total weight of leached impurity $(\mu g)$ : & $<0.24$ & $<0.21$ & $<0.45$ \\
\hline \multirow{2}{*}{$\mathbf{N i}$} & Measured concentration $(\mu \mathrm{g} / \mathrm{ml})$ : & $<8.00 \mathrm{E}-03$ & $<8.00 \mathrm{E}-03$ & Ni \\
\hline & Total weight of leached impurity $(\mu \mathrm{g}):$ & $<1.18$ & $<1.03$ & $<2.21$ \\
\hline \multirow{2}{*}{ Ca } & Measured concentration $(\mu \mathrm{g} / \mathrm{ml})$ : & $<1.00 \mathrm{E}-01$ & $1.54 \mathrm{E}-01$ & $\mathrm{Ca}$ \\
\hline & Total weight of leached impurity $(\mu \mathrm{g})$ : & $<14.70$ & 19.87 & $<34.57$ \\
\hline \multirow{2}{*}{ Al } & Measured concentration $(\mu \mathrm{g} / \mathrm{ml})$ : & $4.92 E-02$ & $8.93 E-03$ & Al \\
\hline & Total weight of leached impurity $(\mu \mathrm{g})$ : & 7.23 & 1.15 & 8.38 \\
\hline \multirow{2}{*}{$\mathbf{T i}$} & Measured concentration $(\mu \mathrm{g} / \mathrm{ml})$ : & $<8.00 \mathrm{E}-03$ & $<8.00 \mathrm{E}-03$ & $\mathrm{Ti}$ \\
\hline & Total weight of leached impurity $(\mu \mathrm{g})$ : & $<1.18$ & $<1.03$ & $<2.21$ \\
\hline \multirow{2}{*}{ v } & Measured concentration $(\mu \mathrm{g} / \mathrm{ml})$ : & $<2.00 \mathrm{E}-03$ & $<2.00 \mathrm{E}-03$ & v \\
\hline & Total weight of leached impurity $(\mu \mathrm{g})$ : & $<0.29$ & $<0.26$ & $<0.55$ \\
\hline
\end{tabular}

Comments

Data checked against RMAL 2522 official results of analyses by FCM on 2/01/2010. 7red c. Montgomery
$2-11-2010$ 
Data Report Form DRF-26B: Measurement of SIC Burn-Leach Defects or Impurities by Burn-Leach

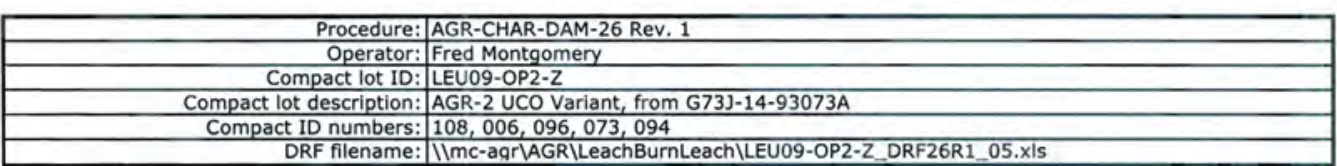

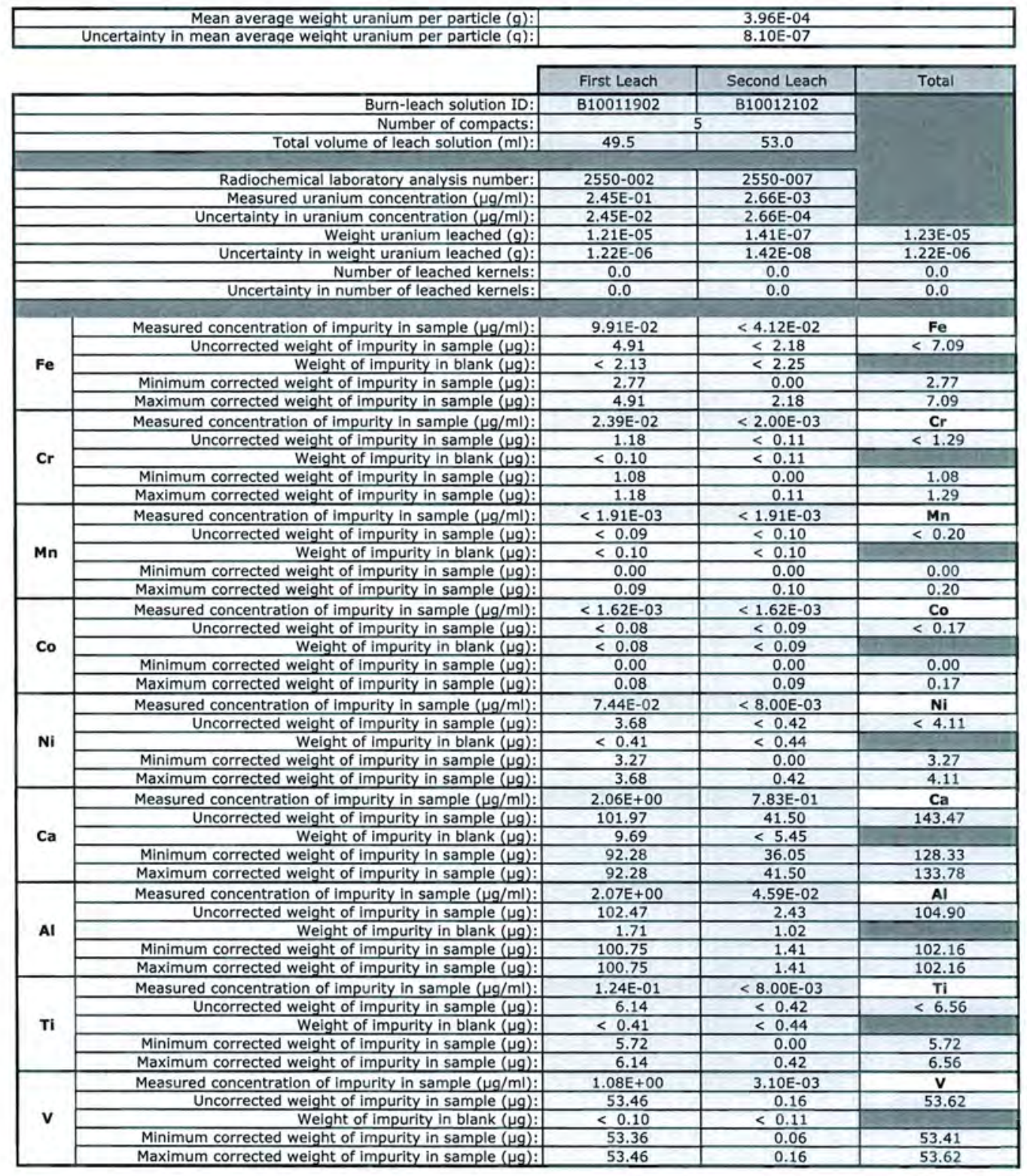

\section{Comments}

First leach for calcium was re-analyzed due to possible Ca contamination during initial analysis. Initial measurement per RMAL 2550002 was $3.50 \mathrm{ug} / \mathrm{ml}$. Re-analysis result entered into table above was obtained per RMAL 2579.

Data checked against RMAL 2550 official results of analyses by FCM on 1/29/2010 and RMAL 2579 official results of analyses by FCM on $2 / 11 / 2010$.

Fred c. Montgo mey
$2-11-2010$ 
Data Report Form DRF-26B: Measurement of SIC Burn-Leach Defects or Impurities by Burn-Leach

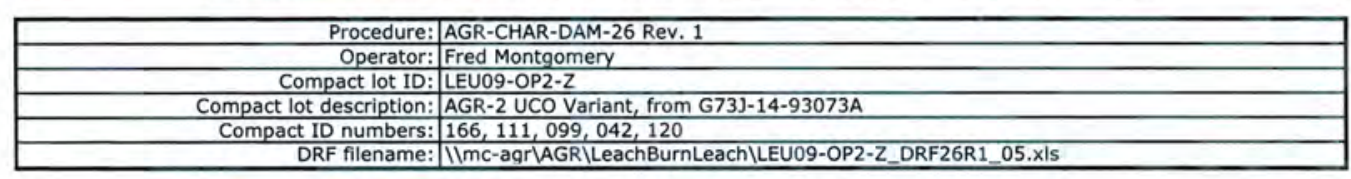

\begin{tabular}{|c|c|c|c|c|}
\hline \multicolumn{2}{|r|}{$\begin{array}{c}\text { Mean average weight uranium per particle }(\mathrm{g}): \\
\text { Uncertainty in mean average weight uranium per particle }(\mathrm{q}):\end{array}$} & \multicolumn{3}{|c|}{$\begin{array}{l}3.96 E-04 \\
8.10 E-07 \\
\end{array}$} \\
\hline & & First Leach & Second Leach & $\overline{\text { Total }}$ \\
\hline & Burn-leach solution ID: & $B 10011903$ & $B 10012103$ & \\
\hline & Number of compacts: & & & \\
\hline & Total volume of leach solution $(\mathrm{ml})$ : & 48.5 & 51.9 & \\
\hline & Radiochemical laboratory analysis number: & $2550-003$ & $2550-008$ & \\
\hline & Measured uranium concentration $(\mathrm{\mu g} / \mathrm{ml})$ : & $2.44 \mathrm{E}-01$ & $3.17 \mathrm{E}-03$ & \\
\hline & Uncertainty in uranium concentration $(\mathrm{\mu g} / \mathrm{ml})$ : & $2.44 \mathrm{E}-02$ & $3.17 \mathrm{E}-04$ & \\
\hline & Weight uranium leached $(g)$ : & $1.18 \mathrm{E}-05$ & $1.65 \mathrm{E}-07$ & $1.20 \mathrm{E}-05$ \\
\hline & Uncertainty in weight uranium leached $(g)$ : & $1.19 \mathrm{E}-06$ & $1.66 \mathrm{E}-08$ & $1.19 \mathrm{E}-06$ \\
\hline & Number of leached kernels: & 0.0 & 0.0 & 0.0 \\
\hline & Uncertainty in number of leached kernels: & 0.0 & 0.0 & 0.0 \\
\hline \multirow{5}{*}{$\mathbf{F e}$} & Measured concentration of impurity in sample $(\mu \mathrm{g} / \mathrm{ml})$ : & $9.76 \mathrm{E}-02$ & $<4.12 \mathrm{E}-02$ & \\
\hline & Uncorrected weight of impurity in sample $(\mu g)$ : & 4.73 & $<2.14$ & $<6.87$ \\
\hline & Weight of impurity in blank $(\mu \mathrm{g})$ : & $<2.13$ & $<2.25$ & \\
\hline & Minimum corrected weight of impurity in sample $(\mu g)$ : & 2.60 & 0.00 & 2.60 \\
\hline & Maximum corrected weight of impurity in sample $(\mu g)$ : & 4.73 & 2.14 & 6.87 \\
\hline \multirow{5}{*}{$\mathbf{C r}$} & Measured concentration of impurity in sample $(\mu \mathrm{g} / \mathrm{ml})$ : & $2.06 \mathrm{E}-02$ & $<2.00 \mathrm{E}-03$ & $\mathrm{Cr}_{\mathbf{r}}$ \\
\hline & Uncorrected weight of impurity in sample $(\mu \mathrm{g})$ : & 1.00 & $<0.10$ & $<1.10$ \\
\hline & Weight of impurity in blank $(\mu g)$ : & $<0.10$ & $<0.11$ & \\
\hline & Minimum corrected weight of impurity in sample $(\mu g)$ : & 0.90 & 0.00 & 0.90 \\
\hline & Maximum corrected weight of impurity in sample $(\mu g)$ : & 1.00 & 0.10 & 1.10 \\
\hline \multirow{5}{*}{ Mn } & Measured concentration of impurity in sample $(\mu \mathrm{g} / \mathrm{ml})$ : & $<1.91 \mathrm{E}-03$ & $<1.91 \mathrm{E}-03$ & $\mathrm{Mn}$ \\
\hline & Uncorrected weight of impurity in sample $(\mu \mathrm{g})$ : & $<0.09$ & $<0.10$ & $<0.19$ \\
\hline & $\begin{array}{l}\text { Weight of impurity in blank }(\mu g) \text { : } \\
\text {. }\end{array}$ & $<0.10$ & $<0.10$ & \\
\hline & Minimum corrected weight of impurity in sample $(\mu \mathrm{g})$ : & 0.00 & 0.00 & 0.00 \\
\hline & Maximum corrected weight of impurity in sample $(\mu g)$ : & 0.09 & 0.10 & 0.19 \\
\hline \multirow{5}{*}{ co } & Measured concentration of impurity in sample $(\mu \mathrm{g} / \mathrm{ml})$ : & $<1.62 \mathrm{E}-03$ & $<1.62 \mathrm{E}-03$ & Co \\
\hline & Uncorrected weight of impurity in sample $(\mu g)$ : & $<0.08$ & $<0.08$ & $<0.16$ \\
\hline & Weight of impurity in blank $(\mu g)$ : & $<0.08$ & $<0.09$ & \\
\hline & Minimum corrected weight of impurity in sample $(\mu \mathrm{g})$ : & 0.00 & 0.00 & 0.00 \\
\hline & Maximum corrected weight of impurity in sample $(\mathrm{ug})$ : & 0.08 & 0.08 & 0.16 \\
\hline \multirow{5}{*}{ Ni } & Measured concentration of impurity in sample $(\mu \mathrm{g} / \mathrm{ml})$ : & $3.14 \mathrm{E}-02$ & $<8.00 \mathrm{E}-03$ & $\mathrm{Ni}$ \\
\hline & Uncorrected weight of impurity in sample $(\mu g)$ : & 1.52 & $<0.42$ & $<1.94$ \\
\hline & Weight of impurity in blank $(\mu g)$ : & $<0.41$ & $<0.44$ & \\
\hline & Minimum corrected weight of impurity in sample $(\mu \mathrm{g})$ : & 1.11 & 0.00 & 1.11 \\
\hline & Maximum corrected weight of impurity in sample $(\mu \mathrm{gg})$ : & 1.52 & 0.42 & 1.94 \\
\hline \multirow{5}{*}{$\mathbf{C a}$} & Measured concentration of impurity in sample $(\mu \mathrm{g} / \mathrm{ml})$ : & $1.83 \mathrm{E}+00$ & $1.03 E+00$ & $\mathrm{Ca}$ \\
\hline & Uncorrected weight of impurity in sample $(\mu \mathrm{g})$ : & 88.76 & 53.46 & 142.21 \\
\hline & Weight of impurity in blank $(\mathrm{\mu g})$ : & 9.69 & $<5.45$ & \\
\hline & Minimum corrected weight of impurity in sample $(\mu \mathrm{g})$ : & 79.07 & 48.01 & 127.08 \\
\hline & Maximum corrected weight of impurity in sample $(\mu \mathrm{g})$ : & 79.07 & 53.46 & 132.53 \\
\hline \multirow{5}{*}{ AI } & Measured concentration of impurity in sample $(\mu \mathrm{g} / \mathrm{ml})$ : & $1.80 E+00$ & $4.06 \mathrm{E}-02$ & Al \\
\hline & Uncorrected weight of impurity in sample $(\mu \mathrm{g})$ : & 87.30 & 2.11 & 89.41 \\
\hline & Weight of impurity In blank $(\mu \mathrm{g})$ : & 1.71 & 1.02 & \\
\hline & Minimum corrected weight of impurity in sample $(\mathrm{\mu g})$ : & 85.59 & 1.08 & 86.67 \\
\hline & Maximum corrected weight of impurity in sample ( $(\mathrm{g})$ : & 85.59 & 1.08 & 86.67 \\
\hline \multirow{5}{*}{$\mathbf{T i}$} & Measured concentration of impurity in sample $(\mu \mathrm{g} / \mathrm{ml})$ : & $1.20 \mathrm{E}-01$ & $<8.00 \mathrm{E}-03$ & Ti \\
\hline & Uncorrected weight of impurity in sample $(\mu \mathrm{g})$ : & 5.82 & $<0.42$ & $<6.24$ \\
\hline & Weight of impurity in blank $(\mu \mathrm{g})$ : & $<0.41$ & $<0.44$ & \\
\hline & Minimum corrected weight of impurity in sample $(\mathrm{\mu g})$ : & 5.41 & 0.00 & 5.41 \\
\hline & Maximum corrected weight of impurity in sample $(\mu \mathrm{g})$ : & 5.82 & 0.42 & $\frac{6.42}{6.24}$ \\
\hline \multirow{5}{*}{$\mathbf{v}$} & Measured concentration of impurity in sample $(\mu \mathrm{g} / \mathrm{ml})$ : & $1.04 \mathrm{E}+00$ & $9.43 \mathrm{E}-03$ & v \\
\hline & Uncorrected weight of impurity in sample $(\mu \mathrm{g})$ : & 50.44 & 0.49 & 50.93 \\
\hline & Weight of impurity in blank $(\mu g)$ : & $<0.10$ & $<0.11$ & \\
\hline & Minimum corrected weight of impurity in sample $(\mu \mathrm{g})$ : & 50.34 & 0.38 & 50.72 \\
\hline & Maximum corrected weight of impurity in sample $(\mu \mathrm{g})$ : & 50.44 & 0.49 & 50.93 \\
\hline
\end{tabular}

Comments

First leach for calcium was re-analyzed due to possible Ca contamination during initial analysis. Initial measurement per RMAL 2550003 was $6.52 \mathrm{ug} / \mathrm{ml}$. Re-analysis result entered into table above was obtained per RMAL 2579 .

Data checked against RMAL 2550 official results of analyses by FCM on 1/29/2010 and RMAL 2579 official results of analyses by FCM on $2 / 11 / 2010$.

\section{Fied c. Monteromey $2-11-2010$}




\begin{tabular}{|c|c|}
\hline Procedure: & AGR-CHAR-DAM-26 Rev. 1 \\
\hline $\begin{array}{r}\text { Operator: } \\
\text { Compact lot ID: }\end{array}$ & Fred Montgomery \\
\hline $\begin{array}{c}\text { Compact lot ID: } \\
\text { Compact lot description: }\end{array}$ & LEU09-OP2-Z \\
\hline $\begin{array}{l}\text { Compact lot description: } \\
\text { Compact ID numbers: }\end{array}$ & $\begin{array}{l}\text { AGR-2 UCO Variant, from G73J-14-93073A } \\
101.078,136.087,122\end{array}$ \\
\hline DRF filename: & IImc-agr|AGR\LeachBurnLeach|LEU09-OP2-Z \\
\hline
\end{tabular}

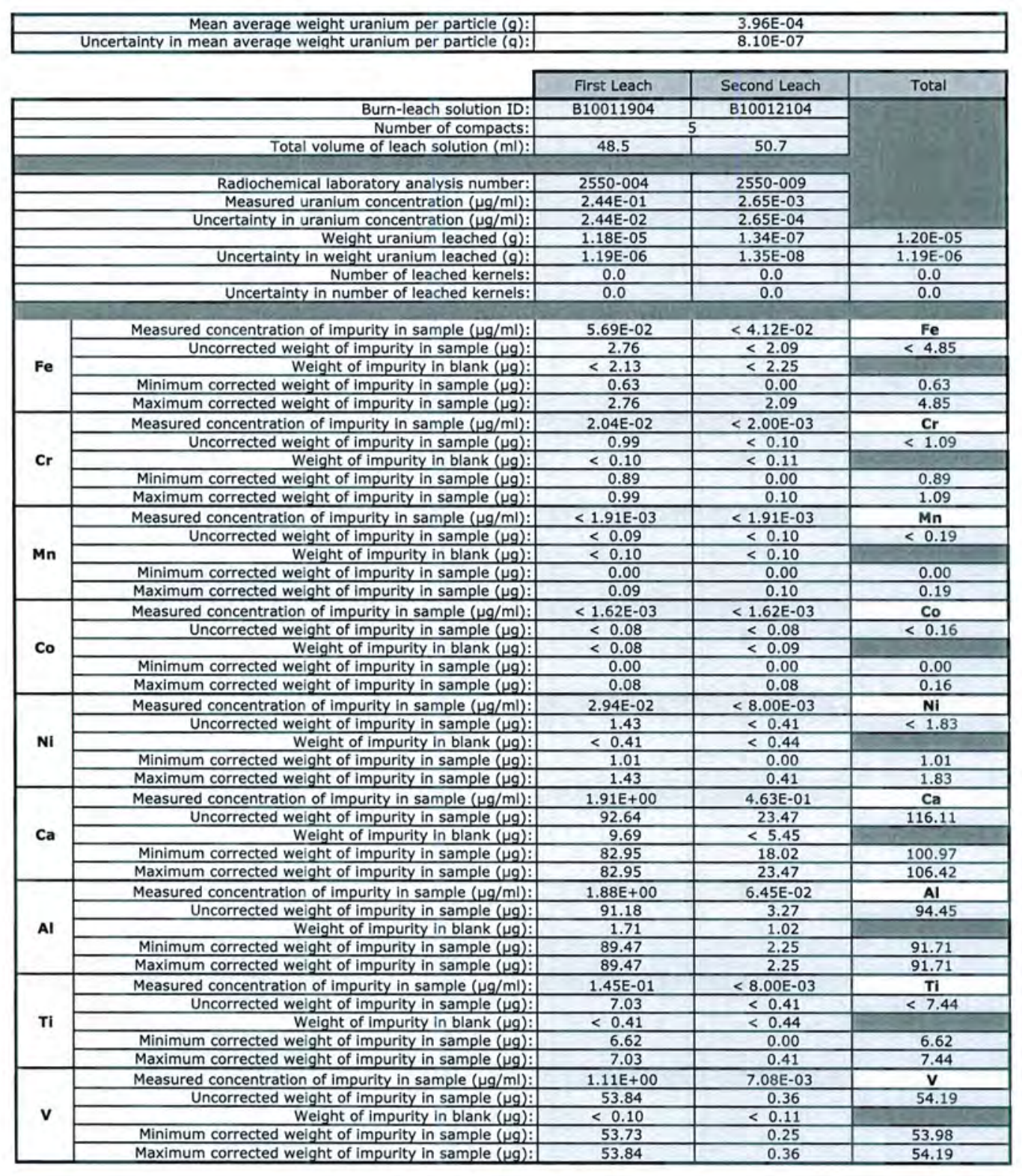

\section{Comments}

First leach for calcium was re-analyzed due to possible Ca contamination during initial analysis. Initial measurement per RMAL 2550004 was $2.22 \mathrm{ug} / \mathrm{ml}$. Re-analysis result entered into table above was obtained per RMAL 2579.

Data checked against RMAL 2550 official results of analyses by FCM on $1 / 29 / 2010$ and RMAL 2579 official results of analyses by FCM on $2 / 11 / 2010$.

\section{$\underset{\substack{\text { Foed c. Montgomery } \\ \text { Opelator }}}{2-11-2010}$}




\begin{tabular}{|c|c|}
\hline Procedure: & AGR-CHAR-DAM-26 Rev, 1 \\
\hline Operator: & Fred Montgomery \\
\hline Compact lot ID: & LEU09-OP2-Z \\
\hline Compact lot description: & AGR-2 UCO Variant, from G73J-14-93073A \\
\hline Compact ID numbers: & $052,146,161,115,095$ \\
\hline DRF filename: & IImc-agr|AGRILeachBurnLeach|LEU09-OP2-Z_DRF26R1_05.xIs \\
\hline
\end{tabular}

\begin{tabular}{|c|c|c|c|c|}
\hline \multirow{2}{*}{\multicolumn{2}{|c|}{$\begin{array}{l}\text { Mean average weight uranium per particle }(g) \text { : } \\
\text { Uncertainty in mean averaqe weight uranium per particle }(q) \text { : }\end{array}$}} & \multicolumn{3}{|c|}{ 3.96E-04 } \\
\hline & & \multicolumn{3}{|c|}{$8.10 \mathrm{E}-07$} \\
\hline & & First Leach & Second Leach & Total \\
\hline & Burn-leach solution ID: & B10011905 & B10012105 & \\
\hline & Number of compacts: & & & \\
\hline & Total volume of leach solution $(\mathrm{ml})$ : & 49.8 & 53.8 & \\
\hline & Radiochemical laboratory analysis number: & $2550-005$ & $2550-010$ & \\
\hline & Measured uranium concentration $(\mu \mathrm{g} / \mathrm{ml})$ : & $2.47 \mathrm{E}-01$ & $1.84 \mathrm{E}-03$ & \\
\hline & Uncertainty in uranium concentration $(\mu \mathrm{g} / \mathrm{ml})$ : & $2.47 \mathrm{E}-02$ & $1.84 \mathrm{E}-04$ & \\
\hline & $\begin{array}{c}\text { Weight uranium leached }(g): \\
\end{array}$ & $1.23 \mathrm{E}-05$ & $9.90 \mathrm{E}-08$ & $1.24 E-05$ \\
\hline & Uncertainty in weight uranium leached $(\mathrm{g})$ : & $1.24 \mathrm{E}-06$ & 9.97E-09 & $1.24 \mathrm{E}-06$ \\
\hline & Number of leached kernels: & 0.0 & 0.0 & 0.0 \\
\hline & Uncertainty in number of leached kernels: & 0.0 & 0.0 & 0.0 \\
\hline \multirow{5}{*}{$\mathbf{F e}$} & Measured concentration of impurity in sample $(\mu \mathrm{g} / \mathrm{ml})$ : & $5.87 E-02$ & $<4.12 \mathrm{E}-02$ & $\mathrm{Fe}$ \\
\hline & Uncorrected weight of impurity in sample $(\mu \mathrm{g})$ : & 2.92 & $<2.22$ & $<5.14$ \\
\hline & Weight of impurity in blank $(\mu \mathrm{g})$ : & $<2.13$ & $<2.25$ & \\
\hline & Minimum corrected weight of impurity in sample $(\mu \mathrm{g})$ : & 0.79 & 0.00 & 0.79 \\
\hline & Maximum corrected weight of impurity in sample $(\mu \mathrm{g})$ : & 2.92 & 2.22 & 5.14 \\
\hline \multirow{5}{*}{$\mathbf{C r}$} & Measured concentration of impurity in sample $(\mu \mathrm{g} / \mathrm{ml})$ : & $2.19 \mathrm{E}-02$ & $<2.00 \mathrm{E}-03$ & $\mathrm{Cr}$ \\
\hline & Uncorrected weight of impurity in sample $(\mu g)$ : & 1.09 & $<0.11$ & $<1.20$ \\
\hline & Weight of impurity in blank $(\mu \mathrm{g})$ : & $<0.10$ & $<0.11$ & \\
\hline & Minimum corrected weight of impurity in sample $(\mu \mathrm{g})$ : & 0.99 & 0.00 & 0.99 \\
\hline & Maximum corrected weight of impurity in sample $(\mu g)$ : & 1.09 & 0.11 & 1.20 \\
\hline \multirow{5}{*}{ Mn } & Measured concentration of impurity in sample $(\mu \mathrm{g} / \mathrm{ml})$ : & $<1.91 \mathrm{E}-03$ & $<1.91 \mathrm{E}-03$ & Mn \\
\hline & Uncorrected weight of impurity in sample $(\mu \mathrm{g})$ : & $<0.10$ & $<0.10$ & $<0.20$ \\
\hline & Weight of impurity in blank $(\mu \mathrm{g})$ : & $<0.10$ & $<0.10$ & \\
\hline & Minimum corrected weight of impurity in sample $(\mu \mathrm{g})$ : & 0.00 & 0.00 & 0.00 \\
\hline & Maximum corrected weight of impurity in sample $(\mu \mathrm{g})$ : & 0.10 & 0.10 & 0.20 \\
\hline \multirow{5}{*}{ Co } & Measured concentration of impurity in sample $(\mu \mathrm{g} / \mathrm{ml})$ : & $<1.62 \mathrm{E}-03$ & $<1.62 \mathrm{E}-03$ & Co \\
\hline & Uncorrected weight of impurity in sample $(\mu \mathrm{g})$ : & $<0.08$ & $<0.09$ & $<0.17$ \\
\hline & Weight of impurity in blank $(\mu \mathrm{g})$ : & $<0.08$ & $<0.09$ & \\
\hline & Minimum corrected weight of impurity in sample $(\mu \mathrm{g})$ : & 0.00 & 0.00 & 0.00 \\
\hline & Maximum corrected weight of impurity in sample $(\mu \mathrm{g})$ : & 0.08 & 0.09 & 0.17 \\
\hline \multirow{5}{*}{$\mathrm{Ni}$} & Measured concentration of impurity in sample $(\mu \mathrm{g} / \mathrm{ml})$ : & $3.64 \mathrm{E}-02$ & $<8.00 \mathrm{E}-03$ & $\mathrm{Ni}$ \\
\hline & Uncorrected weight of impurity in sample $(\mu \mathrm{g})$ : & 1.81 & $<0.43$ & $<2.24$ \\
\hline & Weight of impurity in blank $(\mu \mathrm{g})$ : & $<0.41$ & $<0.44$ & \\
\hline & Minimum corrected weight of impurity in sample $(\mu \mathrm{g})$ : & 1.40 & 0.00 & 1.40 \\
\hline & Maximum corrected weight of impurity in sample $(\mu g)$ : & 1.81 & 0.43 & 2.24 \\
\hline \multirow{5}{*}{$\mathbf{C a}$} & Measured concentration of impurity in sample $(\mu \mathrm{g} / \mathrm{ml})$ : & $1.99 \mathrm{E}+00$ & $6.85 \mathrm{E}-01$ & $\mathbf{C a}$ \\
\hline & Uncorrected weight of impurity in sample $(\mu \mathrm{g})$ : & 99.10 & 36.85 & 135.96 \\
\hline & Weight of impurity in blank $(\mu \mathrm{g})$ : & 9.69 & $<5.45$ & \\
\hline & Minimum corrected weight of impurity in sample $(\mu \mathrm{g})$ : & 89.42 & 31.40 & 120.82 \\
\hline & Maximum corrected weight of impurity in sample $(\mu \mathrm{g})$ : & 89.42 & 36.85 & 126.27 \\
\hline \multirow{5}{*}{ Al } & Measured concentration of impurity in sample $(\mu \mathrm{g} / \mathrm{ml})$ : & $1.85 \mathrm{E}+00$ & $1.26 \mathrm{E}-01$ & Al \\
\hline & Uncorrected weight of impurity in sample $(\mu \mathrm{g})$ : & 92.13 & 6.78 & 98.91 \\
\hline & Weight of impurity in blank $(\mu \mathrm{g})$ : & 1.71 & 1.02 & + \\
\hline & Minimum corrected weight of impurity in sample $(\mu \mathrm{g})$ : & 90.42 & 5.75 & 96.17 \\
\hline & Maximum corrected weight of impurity in sample $(\mu \mathrm{g})$ : & 90.42 & 5.75 & 96.17 \\
\hline \multirow{5}{*}{$\mathbf{T i}$} & Measured concentration of impurity in sample $(\mu \mathrm{g} / \mathrm{ml})$ : & $1.09 \mathrm{E}-01$ & $<8.00 \mathrm{E}-03$ & $\mathbf{T i}$ \\
\hline & Uncorrected weight of impurity in sample $(\mu \mathrm{g})$ : & 5.43 & $<0.43$ & $<5.86$ \\
\hline & Weight of Impurity in blank $(\mu \mathrm{g})$ : & $<0.41$ & $<0.44$ & \\
\hline & Minimum corrected weight of impurity in sample $(\mu \mathrm{g})$ : & 5.01 & 0.00 & 5.01 \\
\hline & Maximum corrected weight of impurity in sample $(\mu \mathrm{g})$ : & 5.43 & 0.43 & 5.86 \\
\hline \multirow{5}{*}{$\mathbf{v}$} & Measured concentration of impurity in sample $(\mu \mathrm{g} / \mathrm{ml})$ : & $9.88 \mathrm{E}-01$ & $5.81 \mathrm{E}-03$ & $\mathbf{v}$ \\
\hline & Uncorrected weight of impurity in sample $(\mu \mathrm{g})$ : & 49.20 & 0.31 & 49.51 \\
\hline & Weight of impurity in blank $(\mu \mathrm{g})$ : & $<0.10$ & $<0.11$ & 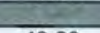 \\
\hline & Minimum corrected weight of impurity in sample $(\mu \mathrm{g})$ : & 49.10 & 0.20 & 49.30 \\
\hline & Maximum corrected weight of impurity in sample $(\mu \mathrm{g})$ : & 49.20 & 0.31 & 49.51 \\
\hline
\end{tabular}

Comments

First leach for calcium was re-analyzed due to possible Ca contamination during initial analysis. Initial measurement per RMAL 2550005 was $3.04 \mathrm{ug} / \mathrm{ml}$. Re-analysis result entered into table above was obtained per RMAL 2579.

Data checked against RMAL 2550 official results of analyses by FCM on 1/29/2010 and RMAL 2579 official results of analyses by FCM on $2 / 11 / 2010$.

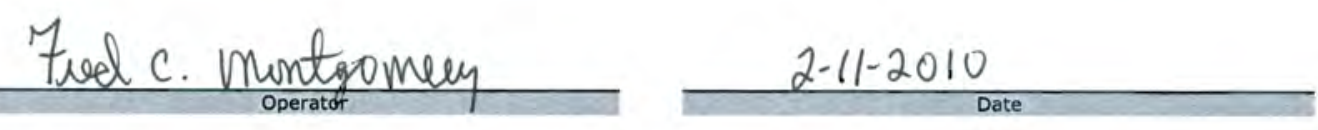


Data Report Form DRF-26B: Measurement of SiC Burn-Leach Defects or Impurities by Burn-Leach

\begin{tabular}{|r|l|}
\hline Procedure: & AGR-CHAR-DAM-26 Rev. 1 \\
\hline Operator: & Fred Montgomery \\
\hline Compact lot ID: & LEU09-OP2-Z \\
\hline Compact lot description: & AGR-2 UCO Variant, from G73J-14-93073A \\
\hline Compact ID numbers: & Burn-Leach Blank \\
\hline DRF filename: & IImC-agr|AGRILeachBurnLeach ILEU09-OP2-Z_DRF26R1_05.xIs \\
\hline
\end{tabular}

\begin{tabular}{|c|c|c|c|c|}
\hline \multirow{2}{*}{\multicolumn{2}{|c|}{$\begin{array}{r}\text { Mean average weight uranium per particle }(\mathrm{g}) \text { : } \\
\text { Uncertainty in mean average weight uranium per particle }(\mathrm{g}): \\
\end{array}$}} & \multicolumn{3}{|c|}{$3.96 \mathrm{E}-04$} \\
\hline & & \multicolumn{3}{|c|}{$8.10 \mathrm{E}-07$} \\
\hline & & First Leach & Second Leach & Total \\
\hline & Burn-leach solution ID: & $\mathrm{B} 10011901$ & $\mathrm{~B} 10012101$ & \\
\hline & Number of compacts: & \multicolumn{2}{|c|}{ None } & \\
\hline & Total volume of leach solution $(\mathrm{ml})$ : & 51.8 & 54.5 & \\
\hline & Radiochemical laboratory analysis number: & $2550-001$ & $2550-006$ & \\
\hline & Measured uranium concentration $(\mu \mathrm{g} / \mathrm{ml})$ : & $<2.00 \mathrm{E}-04$ & $<2.00 \mathrm{E}-04$ & \\
\hline \multicolumn{2}{|r|}{ Uncertainty in uranium concentration $(\mu \mathrm{g} / \mathrm{ml})$ : } & & & \\
\hline & Weight uranium leached $(\mathrm{g})$ : & $<1.04 \mathrm{E}-08$ & $<1.09 \mathrm{E}-08$ & $<2.13 \mathrm{E}-08$ \\
\hline \multicolumn{2}{|r|}{ Uncertainty in weight uranium leached $(\mathrm{g})$ : } & & & \\
\hline \multirow{2}{*}{\multicolumn{2}{|c|}{$\begin{array}{l}\text { Number of leached kernels: } \\
\text { Uncertainty in number of leached kernels: }\end{array}$}} & 0.0 & 0.0 & 0.0 \\
\hline & & & & \\
\hline \multirow{2}{*}{$\mathbf{F e}$} & Measured concentration $(\mu \mathrm{g} / \mathrm{ml})$ : & $<4.12 \mathrm{E}-02$ & $<4.12 \mathrm{E}-02$ & $\mathbf{F e}$ \\
\hline & Total weight of leached impurity $(\mu \mathrm{g})$ : & $<2.13$ & $<2.25$ & $<4.38$ \\
\hline \multirow{2}{*}{$\mathbf{C r}$} & Measured concentration $(\mu \mathrm{g} / \mathrm{ml})$ : & $<2.00 \mathrm{E}-03$ & $<2.00 \mathrm{E}-03$ & $\mathrm{Cr}$ \\
\hline & Total weight of leached impurity $(\mu \mathrm{g})$ : & $<0.10$ & $<0.11$ & $<0.21$ \\
\hline \multirow{2}{*}{ Mn } & Measured concentration $(\mu \mathrm{g} / \mathrm{ml})$ : & $<1.91 \mathrm{E}-03$ & $<1.91 \mathrm{E}-03$ & $\mathrm{Mn}$ \\
\hline & Total weight of leached impurity $(\mu \mathrm{g})$ : & $<0.10$ & $<0.10$ & $<0.20$ \\
\hline \multirow{2}{*}{ Co } & Measured concentration $(\mu \mathrm{g} / \mathrm{ml})$ : & $<1.62 \mathrm{E}-03$ & $<1.62 \mathrm{E}-03$ & Co \\
\hline & Total weight of leached impurity $(\mu \mathrm{g})$ : & $<0.08$ & $<0.09$ & $<0.17$ \\
\hline \multirow{2}{*}{ Ni } & Measured concentration $(\mu \mathrm{g} / \mathrm{ml})$ : & $<8.00 \mathrm{E}-03$ & $<8.00 \mathrm{E}-03$ & $\mathrm{Ni}$ \\
\hline & Total weight of leached impurity $(\mu \mathrm{g})$ : & $<0.41$ & $<0.44$ & $<0.85$ \\
\hline \multirow{2}{*}{$\mathbf{C a}$} & Measured concentration $(\mu \mathrm{g} / \mathrm{ml})$ : & 1.87E-01 & $<1.00 \mathrm{E}-01$ & $\mathrm{Ca}$ \\
\hline & Total weight of leached impurity $(\mu \mathrm{g})$ : & 9.69 & $<5.45$ & $<15.14$ \\
\hline \multirow{2}{*}{ AI } & Measured concentration $(\mu \mathrm{g} / \mathrm{ml})$ : & $3.31 \mathrm{E}-02$ & $1.88 \mathrm{E}-02$ & Al \\
\hline & Total weight of leached impurity $(\mu \mathrm{g})$ : & 1.71 & 1.02 & 2.74 \\
\hline \multirow{2}{*}{ Ti } & Measured concentration $(\mu \mathrm{g} / \mathrm{ml})$ : & $<8.00 \mathrm{E}-03$ & $<8.00 \mathrm{E}-03$ & $\mathbf{T i}$ \\
\hline & Total weight of leached impurity $(\mu \mathrm{g})$ : & $<0.41$ & $<0.44$ & $<0.85$ \\
\hline \multirow[b]{2}{*}{ v } & Measured concentration $(\mu \mathrm{g} / \mathrm{ml})$ : & $<2.00 \mathrm{E}-03$ & $<2.00 \mathrm{E}-03$ & v \\
\hline & Total weight of leached impurity $(\mu \mathrm{g})$ : & $<0.10$ & $<0.11$ & $<0.21$ \\
\hline
\end{tabular}

\section{Comments}

First and second leach for calcium were re-analyzed due to possible Ca contamination during initial analysis. Initial measurement per RMAL2550-001 $=2.04 \mathrm{ug} / \mathrm{ml}$ and RMAL2550-006 $=2.10 \mathrm{ug} / \mathrm{ml}$. Re-analysis result entered into table above was obtained per RMAL 2579.

Data checked against RMAL 2550 official results of analyses by FCM on 1/29/2010 and RMAL 2579 official results of analyses by FCM on $2 / 11 / 2010$.

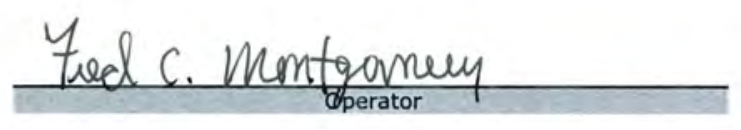
$2-11-2010$

$\frac{2-11-2010}{\text { Date }}$


Data Report Form DRF-27: Counting of Particles with a Defective OPyC Layer from Deconsolidated Compacts by Visual Inspection

\begin{tabular}{|c|c|}
\hline Procedure: & AGR-CHAR-DAM-27 Rev. 0 \\
\hline Operator: & Fred Montgomery \\
\hline Compact lot ID: & LEU09-OP2-Z \\
\hline Compact lot description: & AGR-2 UCO Variant, from G73J-14-93073A \\
\hline Compact ID number: & 091 \\
\hline DRF filename: & IImc-agr|AGR\DefectiveOPyCILEU09-OP2-Z_DRF27R0.xls \\
\hline
\end{tabular}

Number of particles with cracked OPyC: 0

Number of particles with partially missing OPyC:

Number of particles with completely missing OPyC: 0

Total number of particles with defective OPyC: 0

\section{Comments on unusual visual characteristics of OPyC}

Foed c. montromey

$11-17-09$ 
Data Report Form DRF-28: Counting of Particles with Excessive Uranium Dispersion Inside SiC

\begin{tabular}{|r|r|}
\hline Procedure: & AGR-CHAR-DAM-28 Rev. 2 \\
\hline Operator: & John Hunn/Ivan Dunbar/Paul Menchhofer/Chinthaka Silva \\
\hline Compact lot ID: & LEU09-OP2-Z \\
\hline Compact lot description: & AGR-2 UCO Variant Fuel, from G73J-14-93073A \\
\hline Compact ID numbers: & 011152001068046067081019008032039036155012117031149093147072 \\
\hline DRF filename: & |ImC-agr|AGRIDefectiveIPyCILEU09-OP2-Z_DRF28R2.xls \\
\hline
\end{tabular}

Number of compacts from which particles were recovered: 20

\section{Weight of sample of particles $(g): 54.245$}

Number of particles in sample: 63525

Mean average weight/particle (g): $8.54 \mathrm{E}-04$

Number of particles with excessive U dispersion: 0

\section{Comments}

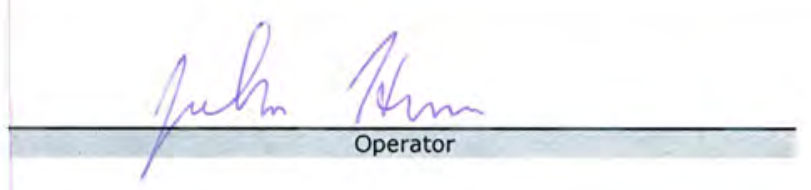

$1-11-10$

Date 


\section{For Information Only}

The information in the remainder of this section is from additional characterization that was not required by the fuel product specification.

\section{Anisotropy of pyrocarbon layers after compacting}

To examine the change in pyrocarbon anisotropy during compact fabrication, particles were recovered after deconsolidation of the particles from the compact for defective OPyC analysis. After compacting, the anisotropy of the pyrocarbon layers was observed to increase. This increase occurs during the heat treatment of the compacts at $1800^{\circ} \mathrm{C}$ for 1 hour. The diattenuation of the IPyC increased from $0.0116 \pm 0.0004$ to $0.0155 \pm 0.0016(1.0349 \pm 0.0012$ to $1.0465 \pm 0.0049$ in terms of effective BAFo). The diattenuation of the OPyC increased from $0.0088 \pm 0.0004$ to $0.0143 \pm 0.0006(1.0263 \pm 0.0011$ to $1.0429 \pm 0.0019$ in terms of effective BAFo). The following data report forms contain the data for these measurements. 
Data Report Form DRF-18A: Measurement of Pyrocarbon Anisotropy using the 2-MGEM - IPyC

Procedure: AGR-CHAR-DAM-18 Rev. 1

Operator: G. E. Jellison

Mount ID: M0911300101

Sample ID: LEU09-OP2-Z091

Sample Description: AGR-2 UCO Variant Fuel, from G73J-14-93073A, after compacting Folder containing data: \\mc-agr\AGR\2-MGEM\R09120901

\begin{tabular}{|c|c|c|c|c|c|c|c|}
\hline \multirow{2}{*}{ Particle \# } & \multirow{2}{*}{$\begin{array}{c}\text { Grid } \\
\text { Position }\end{array}$} & \multicolumn{3}{|c|}{ Diattenuation } & \multicolumn{3}{|c|}{ True $\mathrm{BAFO}=(1+\mathrm{N}) /(1-\mathrm{N})$} \\
\hline & & Average & St. Dev. & Ave. Error & Average & St. Dev. & Ave. Error \\
\hline 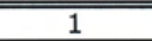 & 4,4 & 0.0144 & 0.0025 & 0.0005 & 1.0292 & 0.0051 & 0.0010 \\
\hline 2 & 4,6 & 0.0116 & 0.0029 & 0.0006 & 1.0235 & 0.0059 & 0.0012 \\
\hline 3 & 5,4 & 0.0167 & 0.0027 & 0.0006 & 1.0340 & 0.0056 & 0.0012 \\
\hline 4 & 5,5 & 0.0169 & 0.0029 & 0.0006 & 1.0344 & 0.0060 & 0.0012 \\
\hline 5 & 5,6 & 0.0144 & 0.0025 & 0.0006 & 1.0292 & 0.0051 & 0.0012 \\
\hline 6 & 6,4 & 0.0161 & 0.0024 & 0.0006 & 1.0327 & 0.0050 & 0.0012 \\
\hline 7 & 6,5 & 0.0167 & 0.0029 & 0.0006 & 1.0340 & 0.0060 & 0.0012 \\
\hline 8 & 6,6 & 0.0161 & 0.0030 & 0.0006 & 1.0327 & 0.0062 & 0.0012 \\
\hline 9 & 7,4 & 0.0161 & 0.0029 & 0.0006 & 1.0327 & 0.0060 & 0.0012 \\
\hline 10 & 7,5 & 0.0159 & 0.0029 & 0.0007 & 1.0323 & 0.0060 & 0.0014 \\
\hline \multicolumn{2}{|c|}{ Average } & 0.0155 & 0.0028 & 0.0006 & 1.0315 & 0.0057 & 0.0012 \\
\hline
\end{tabular}

Mean of average BAFo per particle: 1.0315

Standard deviation of average BAFo per particle: 0.0033

\section{Comments}


Data Report Form DRF-18A: Measurement of Pyrocarbon Anisotropy using the 2-MGEM - IPyC Procedure: AGR-CHAR-DAM-18 Rev. 1

Operator: G. E. Jellison

Mount ID: M0911300101

Sample ID: LEU09-OP2-Z091

Sample Description: AGR-2 UCO Variant Fuel, from G73]-14-93073A, after compacting Folder containing data: \Imc-agr\AGR\2-MGEM\R09120901

\begin{tabular}{|c|c|c|c|c|c|c|c|}
\hline \multirow{2}{*}{ Particle \# } & \multirow{2}{*}{$\begin{array}{c}\text { Grid } \\
\end{array}$} & Position & \multicolumn{3}{|c|}{ Diattenuation } & \multicolumn{3}{c|}{ Equivalent BAFo $=1+3 \mathrm{~N}$} \\
\cline { 3 - 8 } & Average & St. Dev. & Ave. Error & Average & \multicolumn{2}{c|}{ St. Dev. } & Ave. Error \\
\hline \hline 1 & 4,4 & 0.0144 & 0.0025 & 0.0005 & 1.0432 & 0.0075 & 0.0015 \\
\hline 2 & 4,6 & 0.0116 & 0.0029 & 0.0006 & 1.0348 & 0.0087 & 0.0018 \\
\hline 3 & 5,4 & 0.0167 & 0.0027 & 0.0006 & 1.0501 & 0.0081 & 0.0018 \\
\hline 4 & 5,5 & 0.0169 & 0.0029 & 0.0006 & 1.0507 & 0.0087 & 0.0018 \\
\hline 5 & 5,6 & 0.0144 & 0.0025 & 0.0006 & 1.0432 & 0.0075 & 0.0018 \\
\hline 6 & 6,4 & 0.0161 & 0.0024 & 0.0006 & 1.0483 & 0.0072 & 0.0018 \\
\hline 7 & 6,5 & 0.0167 & 0.0029 & 0.0006 & 1.0501 & 0.0087 & 0.0018 \\
\hline 8 & 6,6 & 0.0161 & 0.0030 & 0.0006 & 1.0483 & 0.0090 & 0.0018 \\
\hline 9 & 7,4 & 0.0161 & 0.0029 & 0.0006 & 1.0483 & 0.0087 & 0.0018 \\
\hline 10 & 7,5 & 0.0159 & 0.0029 & 0.0007 & 1.0477 & 0.0087 & 0.0021 \\
\hline \multicolumn{2}{|c|}{ Average } & 0.0155 & 0.0028 & 0.0006 & 1.0465 & 0.0083 & 0.0018 \\
\hline
\end{tabular}

Mean of average BAFo per particle: 1.0465

Standard deviation of average BAFo per particle: 0.0049

\section{Comments}

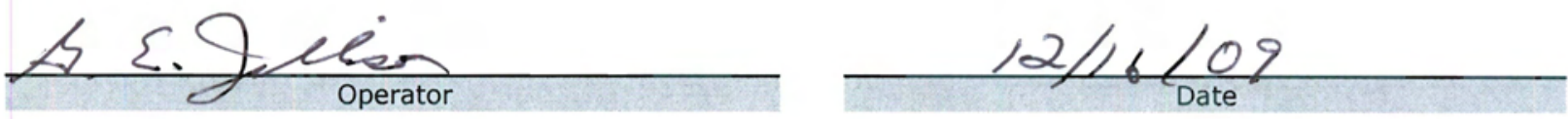


ORNL/TM-2010/017

Data Report Form DRF-18B: Measurement of Pyrocarbon Anisotropy using the 2-MGEM - OPyC Procedure: AGR-CHAR-DAM-18 Rev. 1 Operator: G. E. Jellison

\begin{tabular}{r|l} 
Mount ID: & M0911300101 \\
Sample ID: & LEU09-OP2-Z091
\end{tabular}

Sample Description: AGR-2 UCO Variant Fuel, from G73J-14-93073A, after compacting

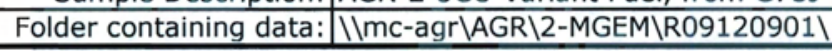

\begin{tabular}{|c|c|c|c|c|c|c|c|}
\hline \multirow{2}{*}{ Particle \# } & \multirow{2}{*}{$\begin{array}{c}\text { Grid } \\
\text { Position }\end{array}$} & \multicolumn{3}{|c|}{ Diattenuation } & \multicolumn{3}{c|}{ True BAFo $=(1+\mathrm{N}) /(1-\mathrm{N})$} \\
\cline { 3 - 8 } & Average & St. Nev. & Ave. Error & Average & \multicolumn{1}{c|}{ St. Lev. } & Ave. Error \\
\hline \hline 1 & 4,4 & 0.0138 & 0.0030 & 0.0007 & 1.0280 & 0.0062 & 0.0014 \\
\hline 2 & 4,6 & 0.0130 & 0.0031 & 0.0008 & 1.0263 & 0.0064 & 0.0016 \\
\hline 3 & 5,4 & 0.0146 & 0.0031 & 0.0008 & 1.0296 & 0.0064 & 0.0016 \\
\hline 4 & 5,5 & 0.0146 & 0.0035 & 0.0007 & 1.0296 & 0.0072 & 0.0014 \\
\hline 5 & 5,6 & 0.0137 & 0.0031 & 0.0007 & 1.0278 & 0.0064 & 0.0014 \\
\hline 6 & 6,4 & 0.0149 & 0.0032 & 0.0008 & 1.0303 & 0.0066 & 0.0016 \\
\hline 7 & 6,5 & 0.0145 & 0.0028 & 0.0008 & 1.0294 & 0.0058 & 0.0016 \\
\hline 8 & 6,6 & 0.0141 & 0.0031 & 0.0008 & 1.0286 & 0.0064 & 0.0016 \\
\hline 9 & 7,4 & 0.0150 & 0.0032 & 0.0008 & 1.0305 & 0.0066 & 0.0016 \\
\hline 10 & 7,5 & 0.0147 & 0.0031 & 0.0008 & 1.0298 & 0.0064 & 0.0016 \\
\hline \multicolumn{2}{r}{ Average } & 0.0143 & 0.0031 & 0.0008 & 1.0290 & 0.0064 & 0.0016 \\
\hline
\end{tabular}

Mean of average BAFo per particle: 1.0290

Standard deviation of average BAFo per particle: 0.0013

Comments
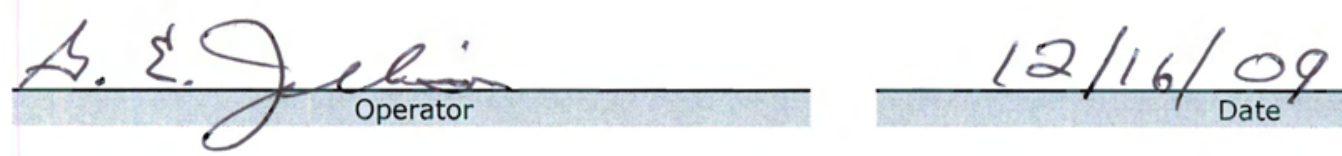

121 
Data Report Form DRF-18B: Measurement of Pyrocarbon Anisotropy using the 2-MGEM - OPyC

\begin{tabular}{|r|l|}
\hline Procedure: & AGR-CHAR-DAM-18 Rev. 1 \\
\hline Operator: & G. E. Jellison \\
\hline Mount ID: & M0911300101 \\
\hline Sample ID: & LEU09-OP2-Z091 \\
\hline Sample Description: & AGR-2 UCO Variant Fuel, from G73J-14-93073A, after compacting \\
\hline Folder containing data: & \Imc-agr\AGR\2-MGEM\R09120901\} $\\
{\hline}$
\end{tabular}

\begin{tabular}{|c|c|c|c|c|c|c|c|}
\hline \multirow{2}{*}{ Particle \# } & \multirow{2}{*}{$\begin{array}{c}\text { Grid } \\
\text { Position }\end{array}$} & \multicolumn{3}{|c|}{ Diattenuation } & \multicolumn{3}{|c|}{ Equivalent $\mathrm{BAFo}=1+3 \mathrm{~N}$} \\
\hline & & Average & St. Dev. & Ave. Error & Average & St. Dev. & Ave. Error \\
\hline 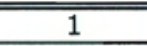 & 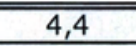 & 0.0138 & 0.0030 & 0.0007 & 1.0414 & 0.0090 & 0.0021 \\
\hline 2 & 4,6 & 0.0130 & 0.0031 & 0.0008 & 1.0390 & 0.0093 & 0.0024 \\
\hline 3 & 5,4 & 0.0146 & 0.0031 & 0.0008 & 1.0438 & 0.0093 & 0.0024 \\
\hline 4 & 5,5 & 0.0146 & 0.0035 & 0.0007 & 1.0438 & 0.0105 & 0.0021 \\
\hline 5 & 5,6 & 0.0137 & 0.0031 & 0.0007 & 1.0411 & 0.0093 & 0.0021 \\
\hline 6 & 6,4 & 0.0149 & 0.0032 & 0.0008 & 1.0447 & 0.0096 & 0.0024 \\
\hline 7 & 6,5 & 0.0145 & 0.0028 & 0.0008 & 1.0435 & 0.0084 & 0.0024 \\
\hline 8 & 6,6 & 0.0141 & 0.0031 & 0.0008 & 1.0423 & 0.0093 & 0.0024 \\
\hline 9 & 7,4 & 0.0150 & 0.0032 & 0.0008 & 1.0450 & 0.0096 & 0.0024 \\
\hline 10 & 7,5 & 0.0147 & 0.0031 & 0.0008 & 1.0441 & 0.0093 & 0.0024 \\
\hline \multicolumn{2}{|c|}{ Average } & 0.0143 & 0.0031 & 0.0008 & 1.0429 & 0.0094 & 0.0023 \\
\hline
\end{tabular}

Mean of average BAFo per particle: 1.0429

Standard deviation of average BAFo per particle: 0.0019

\section{Comments}

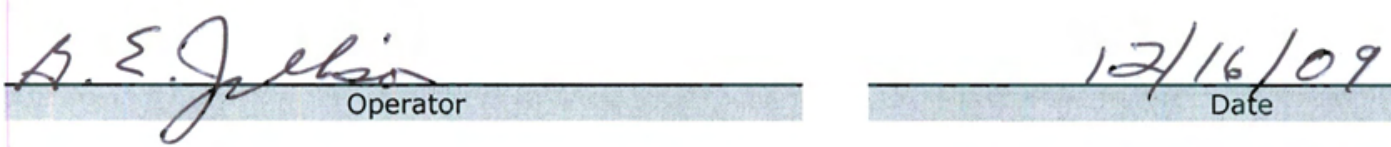




\section{Appendix A: Certificate of Conformance}

This section contains the Certificate of Conformance for the LEU09-OP2-Z compact lot, This is a record of the review by Quality Assurance personnel that specified requirements have been met or that nonconformances to those requirements have been documented. Appendix B contains copies of the applicable Nonconformance Reports. 


\section{Advanced Gas Reactor Fuel Development and Qualification Program \\ CERTIFICATE OF CONFORMANCE}

\section{ITEM IDENTIFICATION: AGR Fuel Compacts}

2. PART LOT AND LOT NUMBER: AGR-2 UCO Variant, LEU09-OP2-Z

3. PRODUCT DEFINITION: INL Document \#SPC-923, Revision 3 entitled AGR-2 Fuel Specification

4. LIST OF APPROVED DEVIATIONS: Not applicable

\begin{tabular}{|c|c|c|c|c|c|c|c|c|c|}
\hline $\begin{array}{l}\text { *Part } \\
\text { Type }\end{array}$ & $\begin{array}{c}\text { Unique Part } \\
\text { I.D. No. }\end{array}$ & QTY & INIT. & Date & $\begin{array}{l}* \text { Part } \\
\text { Type }\end{array}$ & $\begin{array}{c}\text { Unique Part } \\
\text { I.D. No. }\end{array}$ & QTY & INIT. & Date \\
\hline $\mathrm{FC}$ & 002 & 1 & & & $\mathrm{FC}$ & 089 & 1 & & \\
\hline $\mathrm{FC}$ & 004 & 1 & & & FC & 092 & 1 & & \\
\hline $\mathrm{FC}$ & 005 & 1 & & & $\mathrm{FC}$ & 100 & 1 & & \\
\hline $\mathrm{FC}$ & 014 & 1 & & & $\mathrm{FC}$ & 102 & 1 & & \\
\hline $\mathrm{FC}$ & 015 & 1 & & & $\mathrm{FC}$ & 103 & 1 & & \\
\hline $\mathrm{FC}$ & 016 & 1 & & & $\mathrm{FC}$ & 104 & 1 & & \\
\hline $\mathrm{FC}$ & 017 & 1 & & & $\mathrm{FC}$ & 105 & 1 & & \\
\hline $\mathrm{FC}$ & 018 & 1 & & & $\mathrm{FC}$ & 112 & 1 & & \\
\hline FC & 022 & 1 & & & FC & 114 & 1 & & \\
\hline $\mathrm{FC}$ & 026 & 1 & & & $\mathrm{FC}$ & 119 & 1 & & \\
\hline $\mathrm{FC}$ & 028 & 1 & & & $\mathrm{FC}$ & 124 & 1 & & \\
\hline $\mathrm{FC}$ & 038 & 1 & & & $\mathrm{FC}$ & 125 & 1 & & \\
\hline $\mathrm{FC}$ & 040 & 1 & & & $\mathrm{FC}$ & 126 & 1 & & \\
\hline $\mathrm{FC}$ & 043 & 1 & & & $\mathrm{FC}$ & 127 & 1 & & \\
\hline $\mathrm{FC}$ & 049 & 1 & & & $\mathrm{FC}$ & 128 & 1 & & \\
\hline $\mathrm{FC}$ & 053 & 1 & & & $\mathrm{FC}$ & 129 & 1 & & \\
\hline $\mathrm{FC}$ & 056 & 1 & & & $\mathrm{FC}$ & 130 & 1 & & \\
\hline $\mathrm{FC}$ & 057 & 1 & & & $\mathrm{FC}$ & 131 & 1 & & \\
\hline $\mathrm{FC}$ & 059 & 1 & & & $\mathrm{FC}$ & 132 & 1 & & \\
\hline $\mathrm{FC}$ & 062 & 1 & & & $\mathrm{FC}$ & 134 & 1 & & \\
\hline $\mathrm{FC}$ & 063 & 1 & & & $\mathrm{FC}$ & 135 & 1 & & \\
\hline $\mathrm{FC}$ & 066 & 1 & & & $\mathrm{FC}$ & 139 & 1 & & \\
\hline $\mathrm{FC}$ & 069 & 1 & & & $\mathrm{FC}$ & 141 & 1 & & \\
\hline FC & 075 & 1 & & & $\mathrm{FC}$ & 142 & 1 & & \\
\hline $\mathrm{FC}$ & 077 & 1 & & & $\mathrm{FC}$ & 153 & 1 & & \\
\hline $\mathrm{FC}$ & 079 & 1 & & & $\mathrm{FC}$ & 154 & 1 & & \\
\hline $\mathrm{FC}$ & 082 & 1 & & & $\mathrm{FC}$ & 156 & 1 & & \\
\hline $\mathrm{FC}$ & 083 & 1 & & & $\mathrm{FC}$ & 158 & 1 & & \\
\hline $\mathrm{FC}$ & 085 & 1 & & & $\mathrm{FC}$ & 165 & 1 & & \\
\hline $\mathrm{FC}$ & 088 & 1 & & & $\mathrm{FC}$ & 167 & 1 & & \\
\hline
\end{tabular}

\section{LIST OF APPLICABLE NONCONFORMANCE REPORT NUMBERS (NCRs attached in} Appendix B of data package): INL NCR 44791

With the exception of the Deviations documented on the forms referenced in Item 4 and the nonconforming conditions documented on Nonconformance Reports referenced in Item 5, the listed parts have been produced and tested in compliance to the requirements of the QAP for the AGR Program at ORNL (Document \# QAP-ORNL-AGR-01), its subordinate implementing procedures, and to the specified product definition prescribed in the document(s) referenced in Item 3.

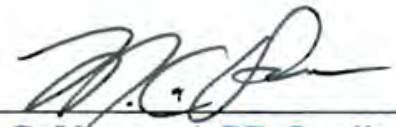

M. C. Vance, AGR Quality Representative,

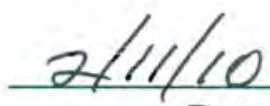

Materials Science and Technology Division, ORNL 


\section{Appendix B: Nonconformance Reports}

This section contains the applicable Nonconformance Reports for the LEU09-OP2-Z compact lot. A nonconformance related to a higher than allowed fraction of exposed uranium was determined by the program to be acceptable for the AGR-2 irradiation test. The exposed uranium was due to cracked TRISO layers in the coated particle composite. These cracks are thought to have occurred at $\mathrm{B} \& \mathrm{~W}$ when particles were removed from the coating furnace using a suction device. The final disposition of this compact lot was to use as is for the AGR-2 irradiation test. This disposition was documented on INL NCR-44791. 
230.01

Revision date

09/30/2003

\section{Control of Nonconforming Items Nonconformance Documentation}
Initlator:
Barnes, Charles M
Work Org.:
C700
Work Phone:
059914
6-0864

Documentation

\begin{tabular}{|c|c|}
\hline $\begin{array}{l}\text { NCR Number: } \\
\mathbf{4 4 7 9 1}\end{array}$ & $\begin{array}{l}\text { Date Identified; } \\
\text { 08/04/2009 }\end{array}$ \\
\hline
\end{tabular}

*Item Name:

LEUO6 compacts containing B\&W G73J-14-93074A particles

and other AGR-2 compact lots (LEU07 and possibly LEUO9

and/or LEU11) containing B\&W AGR-2 particles * SSC:
AGR-2 compacts and coated
particles

\section{*Facility:OFF-S}

*Location:ORNL Description:Bldg 4508 and possibly other ORNL buildings

Req. No/P.O. No./SC and/or Project No.:

Project \#23841; Contract \#27240 with B\&W for Industrial fuel fabrication and development; Contract 59613 with ORNL whlch includes AGR compact fabrication and characterization

Supplier Name/Address:

Supplier of AGR-2 particles is Babcock \&. Wilcox Co., $1570 \mathrm{Mt}$.

Athos Road, Lynchburg, VA 24504

*This NCR is for:

ICP $\triangle I N L \square$ Other

* Is the non-conformance under the requirement of SNF or NRC-licensed activitles (DOE/RW-0333P)? $O$ Yes $O$ No

* Specification to which Item does not perform:

SPC-923, Rev. 2 AGR-2 Fuel Specification (in effect when AGR-2 UCO particle data package was submilted by B\&W) and SPC-923, Rev 3 (In effect when LEU06 and other AGR-2 compacts were characterized)

Associated Documents:

Data Packages for LEU06, LEU07, LEU09 and LEU11 compacts (not issued at this time); B\&W Data Packages for AGR-2 UCO particles, lots G73J-14-93071A, G73J-14-93072A, G73J-14-93073A, G73J-14-93074A \& G73H-10-93085B; TCT meeting notes of March $2 \& 5$ teleconferences, March 16 \& 18 teleconferences, and April 2 teleconference

*Non-Conformance Description:

LEUO6 compacts were found to contain uranium contamination at approximately $10 \mathrm{-4} \mathrm{g}$ exposed $\mathrm{U}$ per gram total $\mathrm{U}$ in compacts, compared to the specification of $\leq 2 \times 10-5 \mathrm{~g}$ exposed $\mathrm{U}$ per $\mathrm{g} U$ in compacts. Exposed uranium lri compacts was determined to be caused by cracks through all layers of the coatings of a fraction of particles contalned in these compacts. Based on several teleconferences of the VHTR TDO Fuels Technlcal Coordination Team (held or March 2,5, 16, 18 and Aprll 2), it was recommended that LEU06 compacts not be used in the AGR-2 experiment because of the high uranlum contamination but replaced by a new set of compacts containing G73J-14-93073A particles. This replacement batch of compacts is expected to have a lower fraction of urantum contamination ( $44 \%$ of the LEU06 fractlon based on all leach and bum leach results and $95 \%$ confidence values or $33 \%$ based on all leach and burn leach results and $50 \%$ confidence values). LEU07 compacts were also found to have uranium contamination above the specification limit, although for a separate reason (urnajum dispersion), LEU07 compacts have been rejected for use in the AGR-2 experiment. The actual uranium contamination values for LEU06 compacts are $\leq 1.4 \mathrm{E}-4$ (95\% confidence based on analysis of 40 compacts only) for LEU06 and $\leqslant 6.9 \times 10-5$ (95\% confidence based on analysis of 100 compacts) for LEU07 compacts. The expecled value for LEU09 compacts is $5 \times 10-5$ (95\% confidence), based on measurements of defect fractions of 217,000 particles from batch $93073 \mathrm{~A}$

\begin{tabular}{ll|l}
\hline Responsible Manager (RM): & Responsible Quality Engineer (QE): \\
Cox, John R & Roberts, Gary D
\end{tabular}

Alternate RM for processing NCR:

Croson, Dlane $\mathrm{V}$

Next Activity: Implementation Completion - RM

Actlonee: Croson, Diane V

Date Due:

Screening - Responsible Manager

\begin{tabular}{l|l|l|l}
\hline $\begin{array}{l}\text { Responsible Manager (RM): } \\
\text { Croson, Diane V }\end{array}$ & $\begin{array}{l}\text { Organlzation } \\
\text { C700 }\end{array}$ & $\begin{array}{l}\text { Phone: } \\
6-3402\end{array}$ & $\begin{array}{l}\text { Date Screened: } \\
12 / 08 / 2009\end{array}$ \\
\hline
\end{tabular}

\footnotetext{
*Initlator has selected "No" to the non-conformance under the requirement of SNF or NRC-licensed activities
} 
(DOE/RW-0333P). Is this Correct?

Yes $O$ No

* Does the NCR require Stop Work?

Yes No

*Does this NCR support Environmental Requirements? $\bigcirc$ Yes No

RM Comments:

None

RM Change History:

12/08/2009 07:26 AM : Angela J Smith changed the RM from Cox, John R to Croson, Diane V

Screening - Quality Engineer

\begin{tabular}{l|l|l|l}
\hline $\begin{array}{l}\text { Quality Engineer (QE): } \\
\text { Roberts, Gary D }\end{array}$ & $\begin{array}{l}\text { Organization } \\
\text { W560: }\end{array}$ & $\begin{array}{l}\text { Date Screened: } \\
12 / 08 / 2009\end{array}$ \\
\hline
\end{tabular}

* is the NCR valid? $O$ Yes $O$ No

Quality Comments:

None

Notification - RM

Responsible Manager (RM):

Croson, Diane $V$

Organization C700

6-8961

$12 / 08 / 2009$

ORPS Report Number:

N/A

*Area of Responsibility:

INL NGNP

Cognizant Director:

Soto, Rafael

Cognizant Director's Alternate(s):

Smith, Angela J; Armour, Kimberly Jo

Compllance Coordinator(s) to determine Price Anderson

(PAAA) noncompliance:

Smith, Angela J

\begin{tabular}{|c|c|}
\hline $\begin{array}{l}\text { Phone: } \\
\text { 6-3402 }\end{array}$ & $\begin{array}{l}\text { Date Notified: } \\
\text { 12/08/2009 }\end{array}$ \\
\hline \multicolumn{2}{|c|}{$\begin{array}{l}\text { * Is the NCR operational equipment needed for Conditional } \\
\text { Use? } \\
\text { YYes No }\end{array}$} \\
\hline \multicolumn{2}{|c|}{ Optional Internal Area of Responsibility: } \\
\hline \multicolumn{2}{|c|}{$\begin{array}{l}{ }^{*} \text { Facility Manager: } \\
\text { Petti, David A }\end{array}$} \\
\hline $\begin{array}{l}\text { *Does } 1 \\
\text { ilems? } \\
\text { Yes }\end{array}$ & ivolve suspect/counterfeit \\
\hline
\end{tabular}

*Does this NCR pertain to Waste Containers, Waste Packaging, or Packaging and Transportation aclivities? $\bigcirc$ Yes No

Method of Segregation:

Material is located at ORNL and is segregated from other fuel batches to prevent inadvertant use

Method of Identification:

Clearly lable by batch number

* Lead Disposition Evaluator:

Barnes, Charles $M$

Additional Disposition Evaluator(s):

(These evaluators verify and concur the disposition of NCR.)

Additional Notification:

\section{QE Red Tag Process}

Quality Engineer (QE):

Roberts, Gary D

Tagging information/Other Methods:

Other means of Tag Identification:

\section{This block is intentionally left blank.}

Disposition

Lead Disposition Evaluator:

Barnes, Charles M

*NCR Disposition:

Use As Is

Repalr

Rework \begin{tabular}{l|l}
\hline Organization: & Phone:
\end{tabular}

\begin{tabular}{l|l}
\hline $\mathrm{C} 700$ & $6-0864$
\end{tabular}

Reject

Multiple Disposition

6-0864
Phone:

6-8961

Date Processed:

$07 / 30 / 2009$
Date Disposition sent for

approval:

$12 / 08 / 2009$

*Multiple Disposition Documentation:

1. LEU06 and LEU07 compacts: Do not use for AGR-2 fuel because of high uranium contamination. However, because full characterization has been performed on these compacts and the kernels and coated particles that they contain, LEUOG 
*Does Disposition represent Design Change? Yes No compacts should be retained in storage at $1 \mathrm{NL}$ and $\mathrm{ORNL}$ for possible future uses. These uses include measurement of thermal conductivity or other compact properties, tests of PIE methods, and tests to better determine fuel specification limits. 2. LEU09 and LEU11 compacts: Use as is. See justification below.

*Does this item require a Unreviewed Safety Question (USQ) screening and evaluation?

Identify as-built drawings and other documentation:( ${ }^{*}$ For Use-As-Is and Repair)

N/A

Method of Disposal:( ${ }^{*}$ For Reject)

N/A

Technical Justification:( ${ }^{*}$ For Use-As-Is and Repair)

Justification for use as is for LEU09 and LEU11 compacts: Uranium equivalent to 2 defective particles has been found in 60 LEU09 compacts; this level is low enough to permil use of these compacts in the AGR-2 experiment, although the level may not meet the fuel uranium contamination specification limit. The final determination of whether LEU09 compacts meet the uranium contamination specification will be made after analyses are complele of ariother 40 compacts. No uranium contamination has been found in the analysls of 40 LEU11 compacts. 60 additlonal LEU11 compacts are being analyzed. Past analyses of multiple sets of 20 compacts show very little variation in results from one set to the next because of the large number of particles in each set of 20 compacts.

Technical requirements and acceptance criteria to be used for repair work:

N/A

Inspections and Verification Criteria for acceptability of repair or rework:

N/A

Other Documents or QA records requiring the change:

N/A

If this nonconforming item is associated with, or caused by, a program, procedure, or process problem, document the issue in accordance with LWP-13840:

N/A

Disposition Concurrence/Approval

\begin{tabular}{l|l}
\hline Approval RM(Signature) & Concurrence/Approval \\
Croson, Diane V & QE(Signature) \\
Diane V Croson & Roberts, Gary D \\
$12 / 08 / 2009$ & Gary D Roberts \\
& $12 / 08 / 2009$ \\
\hline
\end{tabular}

Implementatlon Completion - RM

\begin{tabular}{l|l|l|l}
\hline $\begin{array}{l}\text { Responsible Manager (RM): } \\
\text { Croson, Diane V }\end{array}$ & $\begin{array}{l}\text { Organization } \\
\text { C700 }\end{array}$ & $\begin{array}{l}\text { Phone: } \\
6-3402\end{array}$ & Date Completion: \\
\hline
\end{tabular}

The Disposition as approved has been completed and implemented: $\cup$ Yes $\cup$ N/A

Implementing Documentation:

Attachments/Comments<smiles>[C+]1C2=CC3C1C23</smiles>

PAAA 44791 (AGR-2 compacts).pdf

Revision History 12/08/2009 04:30 PM : Diane V Croson as an RM concurred the Disposition and signed off. 12/08/2009 04:20 PM : Gary D Roberts as a QE concurred the Disposition and signed off. 12/08/2009 02:37 PM : Charles M Barnes completed NCR Disposition and submitted to Croson, Diane V; Roberts, Gary D for their concurrence and approval.

12/08/2009 07:52 AM : Diane V Croson completed Notification Process and notified Roberts, Gary D; Soto, Rafael; Smith, Angela J; Armour, Kimberly Jo; Smith, Angela J; Petti, David A; Barnes, Charles M

12/08/2009 07:44 AM : Gary D Roberts completed screening and forwarded to Croson, Diane V for Notification process.

12/08/2009 07:34 AM : Diane V Croson completed screening and forwarded to Roberts, Gary D for QE Screening.

12/08/2009 07:26 AM : Angela J Smith changed the RM from Cox, John R to Croson, Diane V

08/04/2009 03:26 PM : Charles M Barnes submitted NCR to RM Cox, John R for screening. 
The following fields are general purpose public use. Any data entered here is not related to NCR process and solely used for one's individual need. Integrity of the data is not guarantied since it can be replaced by any user randomly.

FIELD A:

(Field Name: FIELDA, type Text)

FIELD B:

(Field Name: FIELDb, type Text) 


\section{Appendix C: Upgrading of LEU09 using a Roller-micrometer}

As discussed in section 3, a TRISO particle sample was taken from coated particle batch G73J14-93073A, upgraded using a roller-micrometer, and renamed LEU09. LEU09 was used to make the AGR-2 B\&W UCO compact lot LEU09-OP2-Z.

The roller-micrometer technique uses rotating inclined cylinders with a diverging gap to sort particles according to their size. Using a vibrating vee-trough feeder, particles are fed in a single stream into the gap between the rollers. The rollers are angled downward away from the feed point and rotate with an upward and outward motion. Particles travel down the gradually widening gap until they reach a point equal to their width, at which point they drop through the gap into a series of collection bins. The roller-micrometer is a very accurate and reliable device for sorting coated particles by size. It also tends to sort coated particles by shape because the particles continuously re-orient as they travel down the inclined rollers, and faceted particles fall through a narrower gap than spherical particles of the same diameter. Figure $\mathrm{C}-1$ shows the roller-micrometer equipment.

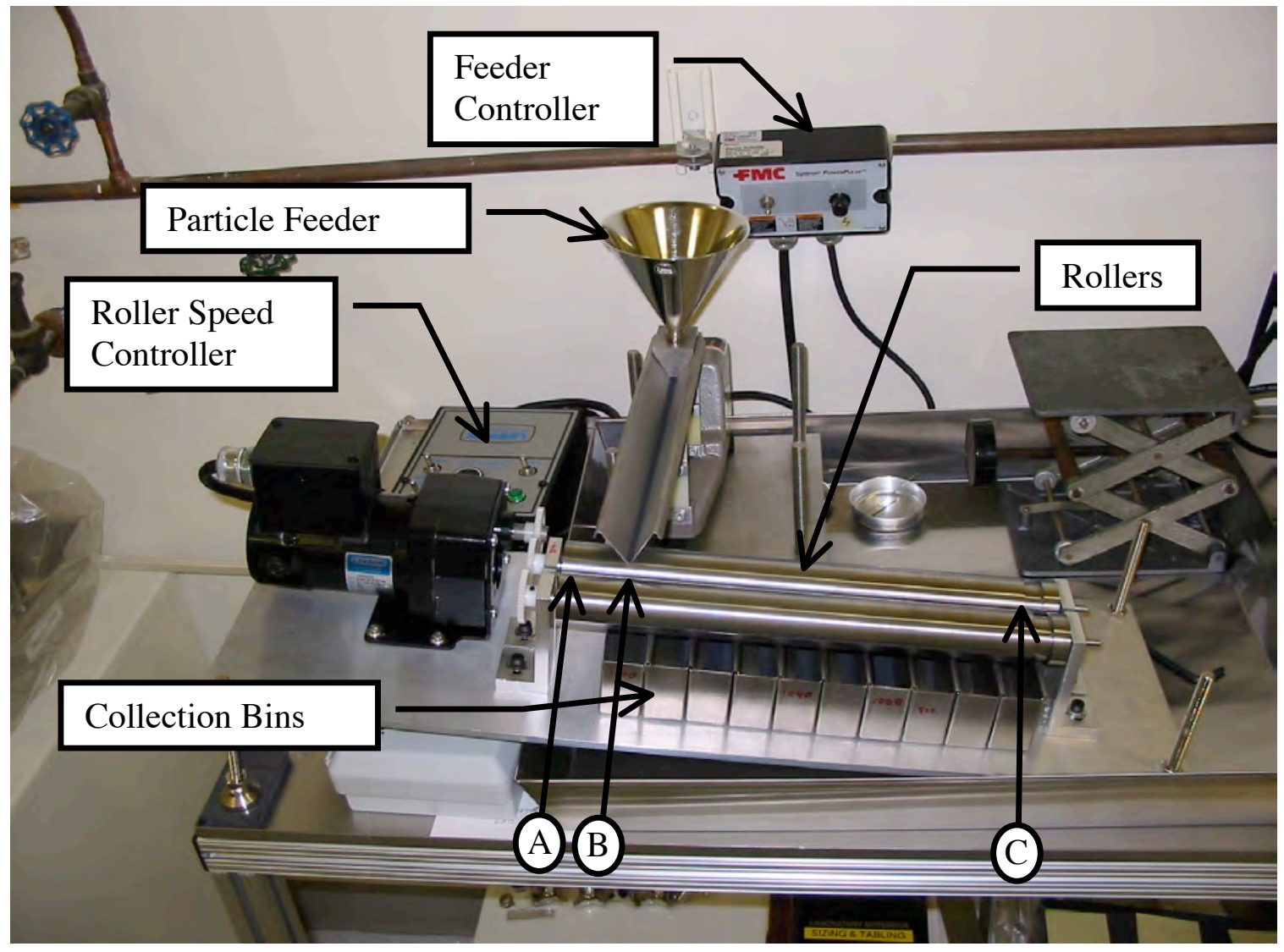

Figure C-1. Photograph of roller-micrometer equipment showing the arrangement of the roller, particle feeder, and collection bins.

The roller gap was adjusted such that particles could be sorted into 11 bins numbered consecutively from 0 to 10 . Each bin spanned a gap width variation of $25 \mu \mathrm{m}$, with Bin 0 at 650 - 
$675 \mu \mathrm{m}$ and Bin 10 at $900 \mu \mathrm{m}$ and above. Table C-1 shows the distribution of the particles after passing through the roller-micrometer. No uncoated kernels or coating fragments were found. Bin 4 contained 14 small or non-spherical particles (Figure C-2). These particles were set aside. Bin 5 contained $\sim 1600$ particles (about $0.16 \%$ of the total population), and some of these particles also exhibited non-spherical shapes (Figure C-3). These particles were not discarded for reasons discussed below. Note that there is also a weight loss reported in Table C-1 due to removal of carbon dust from the surface of the $\mathrm{OPyC}$ and a few particles that bounced off of the rollers during sorting.

Bins 5 - 10 were recombined and the upgraded composite was named LEU09. This decision was based on the fact that the purpose of the roller micrometer upgrading was to separate out and remove uncoated kernels, not to remove the tails of the particle size distribution. The selective removal of the 14 particles in Bin 4 is not expected to impact the results of previously performed sampling and acceptance testing of the kernels and coated particles that went into LEU09. However, further upgrading by removal of Bin 5 or Bin 10 could change the mean properties of the composite to a degree where some of the previous QC data would no longer be relevant. In addition, there is no evidence that the particles in the tails of the roller-micrometer distribution will not perform adequately. Previous observation of undersized particles from a similar coating batch (G73H-10-93087) showed no indication of missing layers and the small diameter was most often related to a thin buffer or, in a few cases, a thin OPyC. Therefore, it was determined by the program that it was desirable to proceed with the irradiation testing of the coated particles as produced by the current $\mathrm{B} \& \mathrm{~W}$ process that did not include roller-micrometer sorting.

Table C-1. Sorting of LEU09 by roller-micrometer

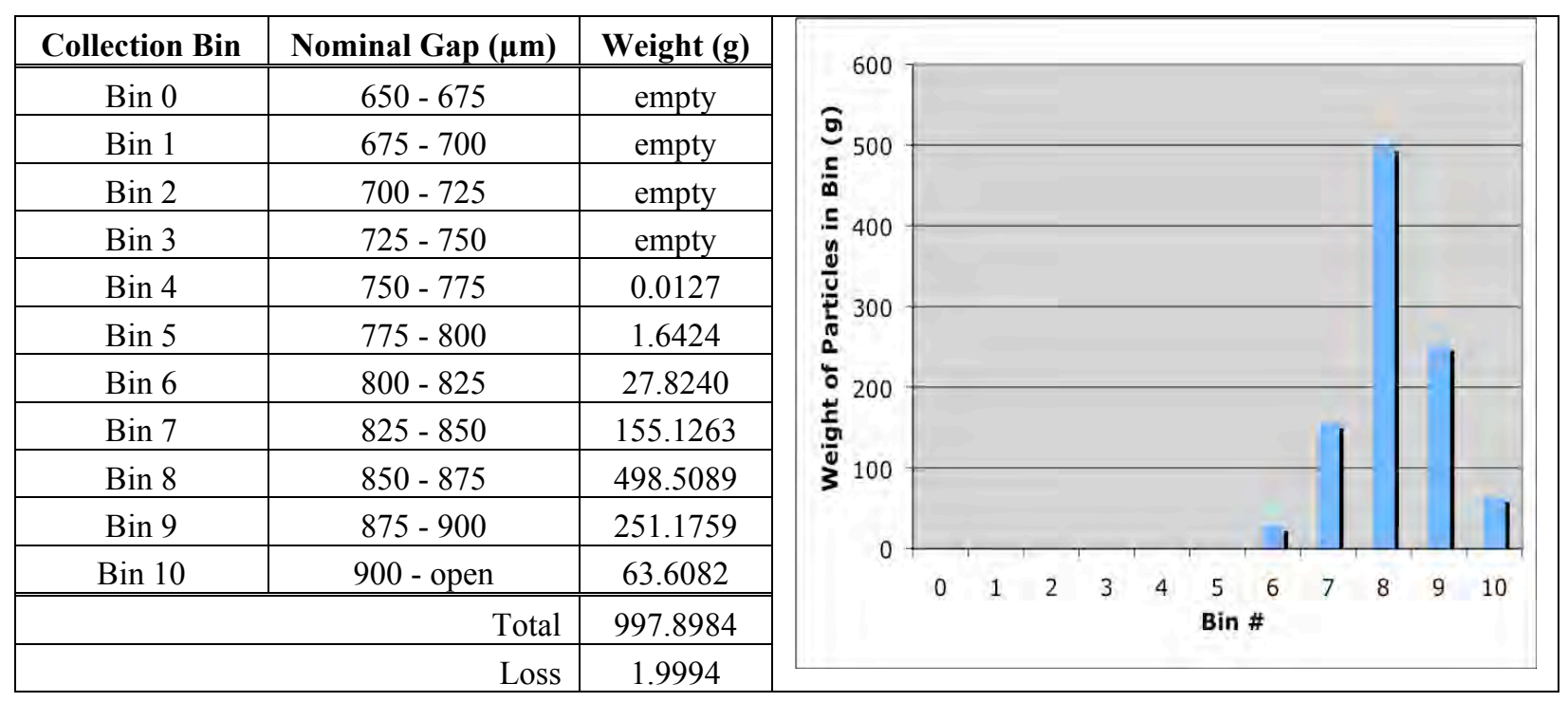




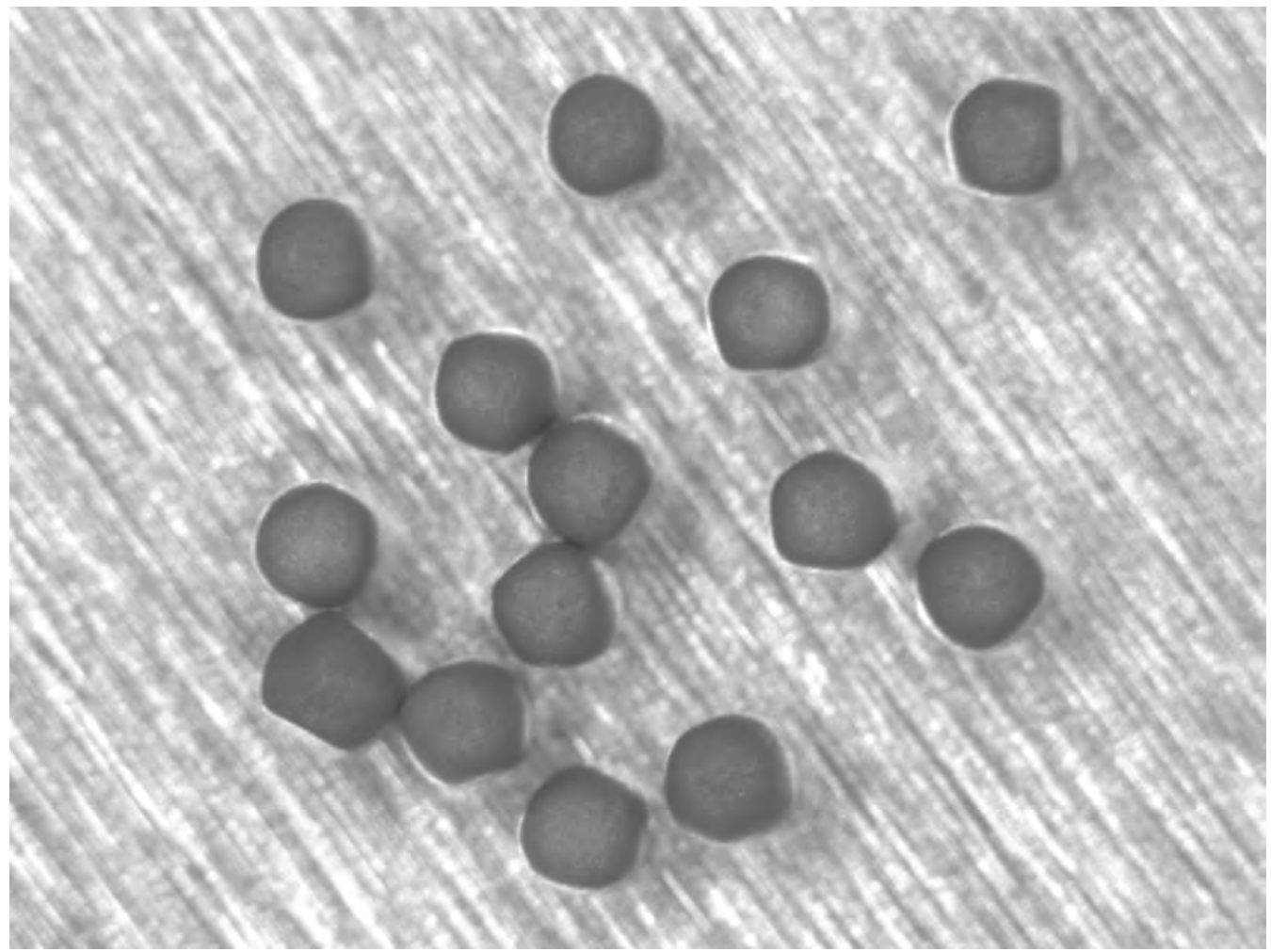

Figure C-2. Photograph of particles from Bin 4.

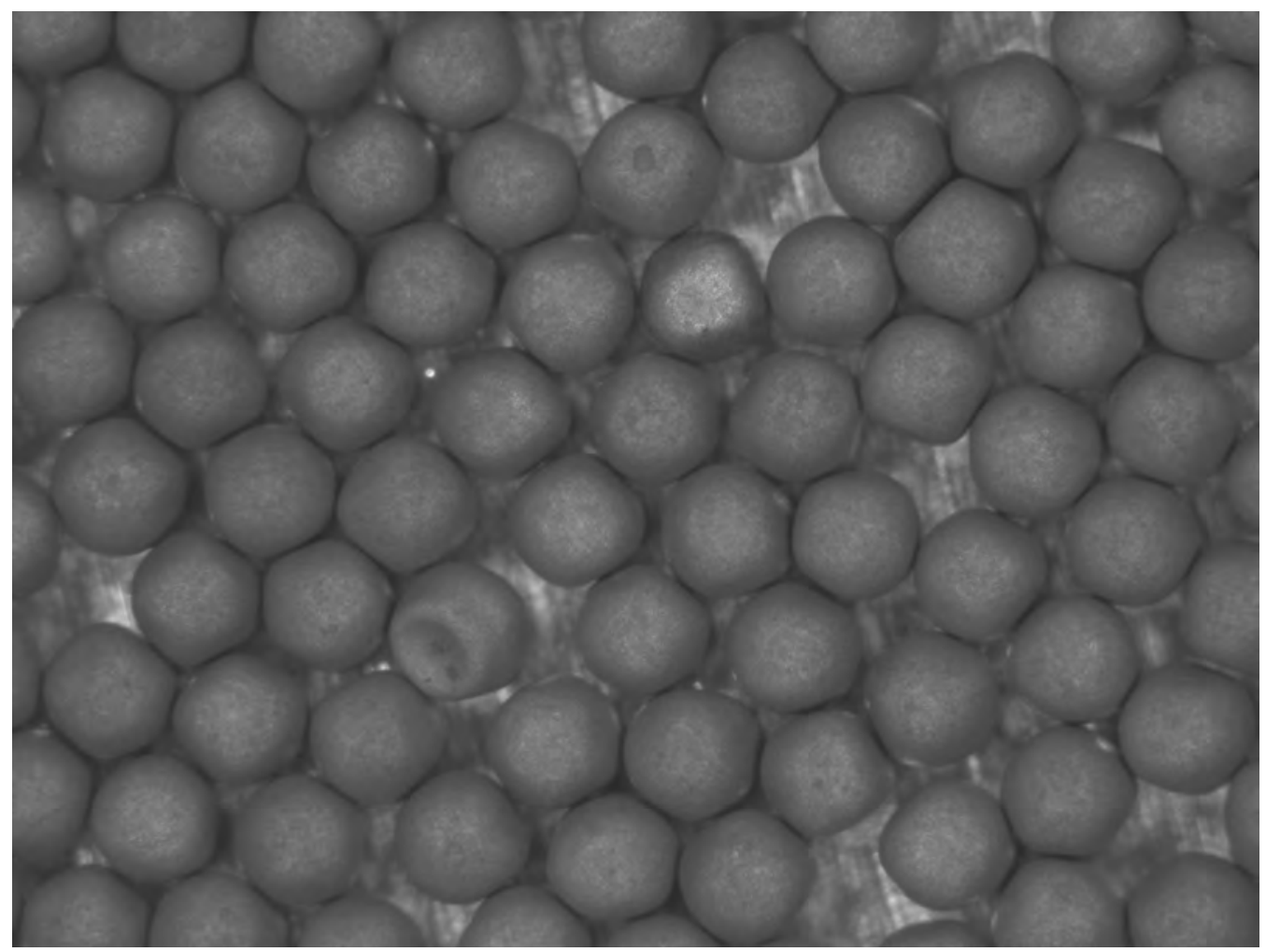

Figure C-3. Photograph of fragment from Bin 5. 